FRANCISCA DANIELE JARDILINO SILAMI

INFLUÊNCIA DOS CIMENTOS RESINOSOS E SISTEMAS CERÂMICOS NA TRANSLUCIDEZ E ESTABILIDADE DE COR DE RESTAURAÇÕES LAMINADAS.

RIBEIRÃO PRETO 



\title{
INFLUÊNCIA DOS CIMENTOS RESINOSOS E SISTEMAS CERÂMICOS NA TRANSLUCIDEZ E ESTABILIDADE DE COR DE RESTAURAÇÕES LAMINADAS.
}

\author{
Tese apresentada à Faculdade de Odontologia de \\ Ribeirão Preto, da Universidade de São Paulo, para \\ a obtenção do título de Doutor em Odontologia. \\ Área de Concentração: Reabilitação Oral. \\ Orientadora: Profa. Dra. Rossana Pereira de \\ Almeida Antunes
}

VERSÃO CORRIGIDA

RIBEIRÃO PRETO

- 2017- 
AUTORIZO A REPRODUÇÃO E DIVULGAÇÃO DO TEOR TOTAL OU PARCIAL

DESTE TRABALHO POR QUALQUER MEIO CONVENCIONAL OU ELETRÔNICO, PARA FINS DE ESTUDO E PESQUISA, DESDE QUE CITADA A FONTE.

\section{FICHA CATALOGRÁFICA}

Elaborada pela Biblioteca Central do Campus USP - Ribeirão Preto

\section{Silami, Francisca Daniele Jardilino}

Influência dos cimentos resinosos e sistemas cerâmicos na translucidez e estabilidade de cor de restaurações laminadas. Ribeirão Preto, 2017.

162 p. : il. ; $30 \mathrm{~cm}$

Tese de Doutorado, apresentada à Faculdade de Odontologia de Ribeirão Preto/USP. Área de Concentração: Reabilitação Oral.

Versão corrigida da Tese. A versão original se encontra disponível na Biblioteca da Unidade que aloja o Programa e na Biblioteca de Teses e Dissertações da USP (PDTD).

Orientador: Antunes, Rossana Pereira de Almeida

1. Estabilidade de cor. 2. Cimentos resinosos. 3. Facetas laminadas. 4. Translucidez; grau de conversão. 5. Linha de cimentação. 
FOLHA DE APROVAÇÃo

Francisca DANIELE JARDILINO SILAMI

INFLUÊNCIA DOS CIMENTOS RESINOSOS E SISTEMAS CERÂMICOS NA TRANSLUCIDEZ E ESTABILIDADE DE COR DE RESTAURAÇÕES LAMINADAS.

Tese apresentada à Faculdade de Odontologia de Ribeirão Preto, da Universidade de São Paulo, para a obtenção do título de Doutor em Odontologia.

Área de Concentração: Reabilitação Oral.

Aprovado em

\section{BANCA EXAMINADORA}

1) $\operatorname{Prof}(a) \cdot \operatorname{Dr}(a)$

Instituição: Assinatura:

2) $\operatorname{Prof}(a) \cdot \operatorname{Dr}(a)$

Instituição: Assinatura:

3) $\operatorname{Prof}(a) \cdot \operatorname{Dr}(a)$

Instituição: Assinatura: 

Dedicatória 



\section{It memória de meu querida amiga Brahim Drubbi Filho}

Dedico esse trabalho a você, por me mostrar a alegria de viver, por ser companheiro, solidário, generoso, humano e acima de tudo levar a felicidade por onde quer que fosse. Sinto muito sua falta, e essa é uma pequena homenagem ao grande amigo que tive e que me ajudou a entrar na vida acadêmica.

Infelizmente você não está aqui para me ver concluir o doutorado, mas nunca me esquecerei da sua ajuda para fazer meu curriculum Lattes numa lan house para que eu pudesse participar do processo seletivo do mestrado. Tampouco esquecerei sua felicidade ao ver que eu tinha passado na prova, e sua frustação logo em seguida, por eu não ter sido classificada.

E mais uma vez, você não me deixou desistir. Incentivou-me a fazer novamente o processo seletivo. Falou com a professora Fernanda sobre mim, e após ela permitir que eu realizasse um estágio no LaABio, participei de outro processo seletivo e fui selecionada para o mestrado. Você defendeu seu mestrado, seguiu sua vida no consultório e eu continuei em busca do sonho de me tornar professora.

Agora, prestes a concluir o doutorado, me lembro de você constantemente, do seu incentivo e torcida, e não posso deixar de agradecer sua importância em toda essa trajetória.

Levarei você para sempre no meu coração e nas minhas memórias até o dia que nos encontrarmos novamente.

Fique com o Pai, olhando sempre por nós! 

Agradecimentas Especiais 



\section{A minha familia, marida e filhos.}

Com vocês pude experimentar a forma mais pura do amor, vocês são, sem dúvida alguma, o que há de melhor em mim e um lindo presente dado por Deus para completar e alegrar a minha vida. Agradeço infinitamente pela paciência e compreensão que tiveram comigo durante esse período. Essa conquista é nossa, meus amores!

\section{tos meus pais}

Obrigada por todo amor, carinho e incentivo. Sempre que enfrento alguma dificuldade, lembro-me do que sempre me diziam "Tudo posso naquele que me fortalece", e assim eu sigo em frente, com a ajuda de Deus como vocês me ensinaram.

\section{It professona Fermanda de Carvalha Panzeri Pires de Souza}

Não tenho palavras para expressar minha gratidão por todo aprendizado e crescimento profissional que me proporcionou. A senhora é, sem dúvida, a responsável por toda minha trajetória na pós-graduação e por tudo que conquistei neste período. Saiba que tenho muito carinho e uma infinita admiração e respeito pela brilhante mestre que sempre me conduziu. Obrigada por todas as oportunidades que me deu e principalmente por acreditar em mim e me fazer acreditar também que tudo é possível com muita dedicação.

\section{It professora Rossana Pereira de Almeida Antumes}

Quero agradecer muito por todo apoio e carinho que me deste. Obrigada pelas palavras de incentivo e por me acolher na clínica e laboratório, por confiar em mim na participação das atividades com seus alunos e me fazer sentir apta para a docência. Acima de tudo, obrigada por me acolher e aceitar minha orientação na reta final do doutorado. 

Agradecimentas 

À Faculdade de Odontologia de Ribeirão Preto (FORP - USP), na pessoa da diretora, Profa. Dra. Léa Assed Bezerra da Silva, pelas oportunidades oferecidas durante o doutorado.

À FAPESP pelo apoio financeiro para realização desta pesquisa.

Ao curso de pós-graduação em Reabilitação Oral, representado pela Prof ${ }^{a}$. Dr ${ }^{a}$. Valéria Oliveira Pagnano de Souza, chefe do departamento e Prof. Dr Ricardo Faria Ribeiro, coordenador, por assegurar nossos direitos e deveres com a pós-graduação.

Aos professores do Departamento de Materiais Dentários e Prótese da Faculdade de Odontologia de Ribeirão Preto, FORP-USP, pelos ensinamentos e conhecimentos compartilhados neste período, pelo apoio, paciência e disposição que sempre tiveram comigo.

A Prof ${ }^{a}$. Dr ${ }^{\text {a }}$ Rossana Pereira de Almeida Antunes, pela supervisão no Programa de Aperfeiçoamento de Ensino (PAE) e monitoria.

À técnica Rafaella Tonani do Laboratório de Materiais Dentários Heitor Panzeri, por toda colaboração e disponibilidade durante a pesquisa, mas principalmente por sua amizade e carinho.

Ao técnico Edson Volta do Laboratório Integrado de Pesquisa em Biocompatibilidade de Materiais (LIPEM), pela ajuda no manuseio da máquina de fadiga termomecânica utilizado em minha pesquisa.

Às secretarias da Pós-Graduação e do Departamento de Materiais Dentários e Prótese: Regiane de Cássia Tirado Damasceno, Fernanda Talita de Freitas e Denise Martins Fontes Gonçalves pela disponibilidade e colaboração em todo processo do curso de doutorado em Reabilitação Oral.

Aos funcionários da Seção de Pós-Graduação da Faculdade de Odontologia de Ribeirão Preto, Isabel Cristina Galino Sola e Mary Possano Carmessano pela presteza nas orientações. 
Aos meus queridos amigos pós-graduação: Marcela Salles, Ana Beatriz Sousa, Carla Cecília Alandia-Roman, Renata de Morais, Suleima do Vale, Karen Pintado, Lourenço Roselino, Maurício Badaró, Maurício Provinciatti, entre outros, pelos maravilhosos momentos compartilhados dentro e fora das salas de aula, pela amizade e carinho que sempre tiveram comigo.

À minha querida equipe do LaABio, Profa. Fernanda, Marta Contente, Sérgio Vicente e Rafaella Tonani obrigada pela acolhida, pelo carinho, pelo estudo, pela troca de experiências e pelos momentos de descontração em nosso dia a dia. Vocês são pessoas especiais e merecem todo meu respeito e admiração, além de muito sucesso.

A toda minha Família, irmãos (a), avós, tios(a), sobrinhos(a), cunhados(a), minha madrasta e minha sogra que estão sempre torcendo por mim.

A todos que direta ou indiretamente contribuíram para a realização deste trabalho.

Meu eterno agradecimento, Deus os abençoe sempre! 
Resuma 

SILAMI, F.D.J. Influência dos cimentos resinosos e sistemas cerâmicos na translucidez e estabilidade de cor de restaurações laminadas. 2017. 162p. Tese (Doutorado em Reabilitação Oral). Faculdade de Odontologia de Ribeirão Preto, Universidade de São Paulo.

\section{RESUMO}

O objetivo desse estudo foi avaliar o grau de conversão, a estabilidade de cor e translucidez de cimentos resinosos para cimentação de laminados cerâmicos em duas espessuras, submetidos a diferentes protocolos de envelhecimento. Foram utilizados 270 incisivos bovinos hígidos, cujas porções radiculares foram cortadas e as porções coronárias foram incluídas em matriz de PVC (25mm de diâmetro) com resina acrílica. As faces vestibulares foram planificadas até a dentina. Foram confeccionados 240 corpos-de-prova em cerâmica prensada (IPS e-max Press e IPS e- maxZirpress, IvoclarVivadent), em duas espessuras (0,5 $\mathrm{mm}$ e 1,0 $\mathrm{mm}$ ), que foram separados aleatoriamente em dois grupos, segundo o tipo de cimento resinoso utilizado para fixação: fotoativado (Variolink II, Ivoclar/Vivadent) ou dual (Rely U 200, 3M ESPE). Após a cimentação, todas as amostras foram mantidas em estufa a $37^{\circ} \mathrm{C}$ por $24 \mathrm{~h}$, após o qual foram realizadas as leituras de cor e translucidez iniciais (Vita EasyShade, VITA). Em seguida, as amostras foram subdivididas em três grupos $(n=10)$ de acordo com o envelhecimento a que foram submetidos: Controle - sem envelhecimento; imersão em água destilada por 1 ano e fadiga termomecânica (1.200.000 ciclos, frequência de $1,3 \mathrm{~Hz}$ e ciclagem térmica de $5^{\circ} \mathrm{C} / 37^{\circ} \mathrm{C} / 55^{\circ} \mathrm{C}$ ). Como controle, foram obtidos 30 corpos-deprova de cada cimento resinoso $(0,5 \mathrm{~mm})$ e 30 dentes foram planificados e submetidos aos mesmos processos de envelhecimento, após o qual leituras finais foram realizadas. Foram analisados também o grau de conversão dos cimentos polimerizados através das restaurações (FTIR) e a linha de cimentação em cada um dos grupos ( $n=3)$, antes e depois do envelhecimento. Para isso, as amostras, selecionadas aleatoriamente, foram seccionadas no sentido longitudinal e analisadas em estereomicroscópio de dupla coordenada (Nikon Measurescope). Os dados de estabilidade de cor $(\Delta \mathrm{E})$, translucidez, grau de conversão e linha de cimentação foram submetidas à análise estatística (3-way ANOVA, Tukey, $p<0,05)$. O cimento autoadesivo apresentou maior grau de conversão que o fotoativado $(p<0,05)$ quando utilizada a cerâmica Zirpress, mas produziu maior alteração de cor $(p<0,05)$ e menor translucidez nas restaurações de E-maxPress de $0,5 \mathrm{~mm}$. A ciclagem termomecânica não foi significante para alterações óticas dos sistemas cerâmicos testados. Conclui-se que a espessura do laminado cerâmico não altera o grau de conversão dos cimentos resinosos e o tipo de cimento resinoso influencia a estabilidade de cor das restaurações cerâmicas quando submetido ao envelhecimento com água destilada.

Palavra Chave: estabilidade de cor; cimentos resinosos; facetas laminadas; translucidez; grau de conversão; linha de cimentação. 

Alstract 

SILAMI, F.D.J. Influence of resin cements luting and ceramic systems in the translucency and color stability of veneers. 2017. 162p. Thesis (PhD, Oral Rehabilitation). School of Dentistry of Ribeirão Preto, University of São Paulo.

\begin{abstract}
The aim of this study is to evaluate the degree of conversion, color stability and translucency of resin cement for luting ceramic veneers with two thicknesses submitted to different aging protocols. Two hundred and seventy bovine incisors were used, which had the root cut, and the coronal were embedded in acrylic resin into PVC rings $(25 \mathrm{~mm}$ diameter). The buccal surface was flattened until dentin. Two hundred and forty pressed ceramic veneers (IPS emax Press and IPS e-max ZirPress, IvoclarVivadent) were obtained, in two thicknesses (0,5 $\mathrm{mm}$ and $1.0 \mathrm{~mm}$ ), that were randomly separated into two groups according to the type of resin cement used for luting: light-cured (Variolink II, Ivoclar / Vivadent) or dual cured (Rely U 200, 3M ESPE). After the cementation, all samples were maintained at $37^{\circ} \mathrm{C}$ for $24 \mathrm{~h}$; after first color and translucency readings (Vita EasyShade, VITA) were made. The samples were separated into 3 groups $(n=10)$ according to the aging that was submitted: control - without aging, immersion in distilled water for one year and thermomechanical cycling (1.200.000 cycles, frequency of $1,3 \mathrm{~Hz}$ and thermal cycling of $5^{\circ} \mathrm{C} / 37^{\circ} \mathrm{C} / 55^{\circ} \mathrm{C}$ ). As the control group, 30 samples of each resin cement $(0,5 \mathrm{~mm})$ and flattened teeth were obtained and submitted to the same aging protocols. After tests, the final color and translucency readings were taken. The degree of conversion of resin cements through veneer restoration, and the cement film thickness $(n=3)$ for each group were analyzed (FTIR), before and after aging. For this, the samples were randomly selected, sectioned longitudinally and examined by a stereomicroscope (Nikon Measurescope). The results of color stability $(\Delta \mathrm{E})$, translucency, degree of conversion and cement film thicknesses were statistically analyzed (3-way, ANOVA, Tukey, $p<0.05)$. The self-adhesive cement showed the higher degree of conversion than light-activated luting cement $(p<0.05)$ when Zirpress ceramic was used, but produced higher color change $(p<0.05)$ and lower translucency in $0.5 \mathrm{~mm} E$-maxPress restorations. The thermomechanical cycling was not significant for optical changes of tested ceramic systems. It was concluded that the thickness of ceramic veneers didn't alter the degree of conversion of resin cement and the type of resin cement has influenced the color stability of ceramic restorations when submitted to water aging.
\end{abstract}

Keywords: color stability; resin cements luting; veneers; translucency; degree conversion; line cimentation 

Lista de Figuras 



\section{LISTA DE FIGURAS}

Figura 1- Dente incluído em anel PVC com resina acrílica.................................. 58

Figura 2- Superfície vestibular do dente cortada em dentina............................... $\quad 58$

Figura 3- Gotejamento de cera em matriz para confecção dos corpos-de-prova... $\quad 59$

Figura 4- Solidificação dos padrões de cera.................................................... 59

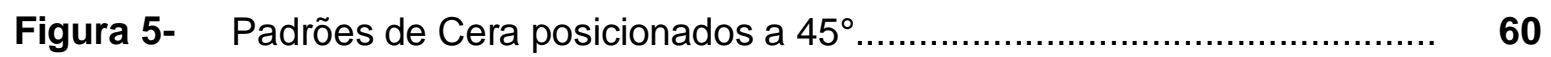

Figura 6- Padrões de cera em anel de silicone para revestimento......................... $\quad 60$

Figura 7- Revestimento posicionado para eliminação da cera.............................. $\quad 61$

Figura 8- Injeção de cerâmica....................................................................... 61

Figura 9- Revestimento resfriado após injeção da cerâmica.................................... 62

Figura 10- Corpos-de-prova de cerâmica após desinclusão..................................... 62

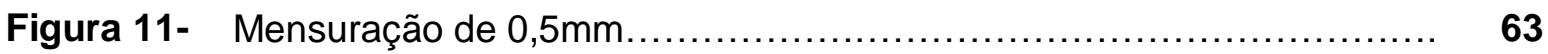

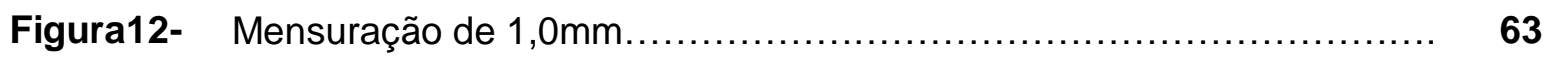

Figura 13- Imagem dos corpos-de-prova finalizados, com glaze............................ 64

Figura 14- Aplicação de ácido fluorídrico.......................................................... 64

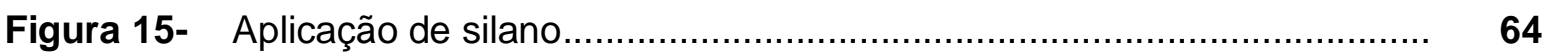

Figura 16- Jateamento com Al2O3

Figura 17- Aplicação de silano............................................................................. 64

Figura 18- Cimento autoadesivo......................................................................... 65

Figura 19- Dosagem do cimento....................................................................... 65

Figura 20- Cimento fotopolimerizável............................................................ 65

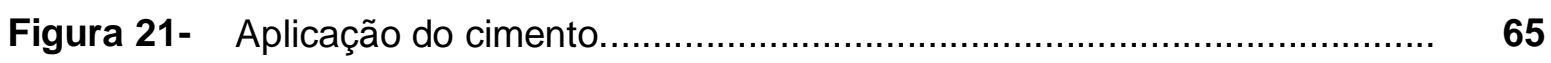

Figura 22- Aplicação ácido fosfórico......................................................... 65

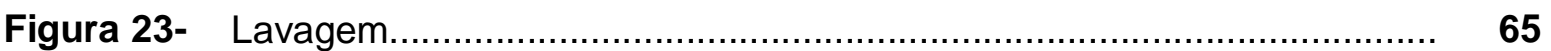

Figura 24- Aplicação do adesivo.......................................................................... 66

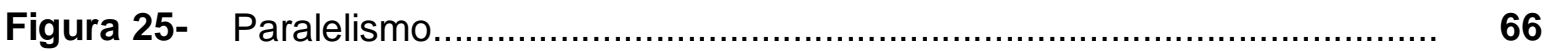

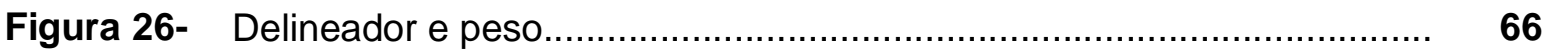

Figura 27- Remoção do excesso e fotoativação do cimento.................................... 66

Figura 28- Vita EasyShade...................................................................... 68

Figura 29- Leitura de cor e translucidez sobre fundo branco.............................. 68

Figura 30- Leitura de translucidez sobre fundo preto............................................ 68

Figura 31- Corpo de prova acondicionado para envelhecimento em água destilada

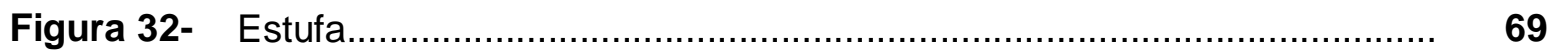

Figura 33- Equipamento de Fadiga termomecânica............................................ 70

Figura 34- Pistão sobre laminado................................................................... 70 
Figura 35- Fibra óptica e espectroradiômetro (Spectroradiometer Model USB 2000, Ocean Optimus, EUA)....................................................... 72

Figura 36- Dados coletados da irradiância espectral............................................. $\quad 72$

Figura 37- Medição do percentual de absorção de luz através da esfera integradora (Illumia, Labsphere, North Sutton, EUA)............................. $\quad 73$

Figura 38- Posicionamento do corpo-de-prova de cerâmica na esfera integradora.. $\quad 73$

Figura 39- Dispositivo FTIR e Linha de Cimentação............................................. $\quad \mathbf{7 4}$

Figura 40- Linha de cimentação posicionada no dispositivo.................................. $\quad \mathbf{7 4}$

Figura 41- Espectrômetro de infravermelho por transformado por Fourier - FTIR (Nicolet 380, ThermoNicolet, EUA)................................................... $\quad \mathbf{7 4}$

Figura 42- Técnica da transmissão................................................................... $\quad \mathbf{7 4}$

Figura 43- Sequência de preparo da amostra..................................................... $\quad 75$

Figura 44- Secção da amostra................................................................... $\quad 75$

Figura 45- Posicionamento da amostra no estereomicroscópio............................... 75

Figura 46- Corpos de prova fraturados.......................................................... 82

Figura 47- Imagem ampliada dos corpos de prova fraturados................................ $\quad \mathbf{8 2}$ 
Lista de Gráficas 



\section{LISTA DE GRÁFICOS}

Gráfico 1- Imagem ilustrativa do percentual de luz através dos materiais e espessuras testados................................................................... 79

Gráfico 2- Alteração de cor dos grupos estudados................................................ $\quad 83$

Gráfico 3- Alteração de Delta L dos grupos estudados...................................... $\quad 85$

Gráfico 4- Alteração de Delta a dos grupos estudados....................................... $\quad 88$

Gráfico 5- Alteração de Delta b dos grupos estudados....................................... 91

Gráfico 6- Alteração de translucidez dos grupos estudados.................................... 94

Gráfico 7- Comparação dos valores de espessura da linha de cimentação dos cimentos estudados. 

Lista de Tabelas 



\section{LISTA DE TABELAS}

Tabela 1- Materiais utilizados no estudo.

Tabela 2- Comparação das médias (3-way ANOVA, Tukey, $p<0,05)$ do grau de conversão dos cimentos resinosos dos para grupos estudados

Tabela 3- Comparação das médias e desvio padrão (3-way ANOVA, teste de Tukey, $p<0,05)$ da alteração de cor dos grupos estudados.

Tabela 4- Comparação das médias e desvio padrão (3-way ANOVA, teste de Tukey, $p<0,05)$ da alteração de Delta $L$ dos grupos estudados.

Tabela 5- Comparação das médias e desvio padrão (3-way ANOVA, teste de Tukey, $\mathrm{p}<0,05)$ da variação do Delta a dos grupos estudados.

Tabela 6- Comparação das médias e desvio padrão (3-way ANOVA, teste de Tukey, $p<0,05)$ da variação do Delta $b$ dos grupos estudados.

Tabela 7- Comparação das médias e desvio padrão (3-way ANOVA, teste de Tukey, $p<0,05)$ da variação de Translucidez dos grupos estudados.........

Tabela 8- Comparação das médias e desvio padrão (3-way ANOVA, teste de Tukey, $\mathrm{p}<0,05)$ da linha de cimentação $(\mu \mathrm{m})$ dos cimentos resinosos dos grupos estudados 

Sumária 

RESUMO

ABSTRACT

LISTA DE FIGURAS

LISTA DE GRÁFICOS

LISTA DE TABELAS

1. INTRODUÇÃO

2. PROPOSIÇÃO

3. MATERIAIS E MÉTODO

3.1 Preparo dos dentes

3.2 Confecção dos corpos-de-prova

3.3 Cimentação dos corpos-de-prova

3.4 Leituras de cor e translucidez $\quad 67$

3.5 Envelhecimento em água destilada $\quad 69$

3.6 Ciclagem termomecânica

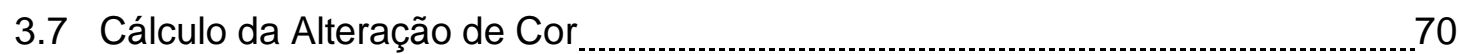

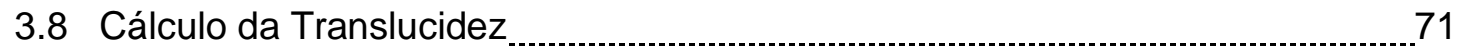

3.9 Grau de conversão 71

3.10 Linha de Cimentação

3.11 Grupos Controles 76

4. RESULTADOS

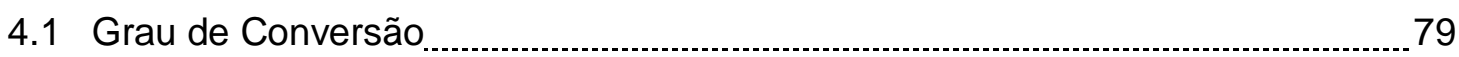

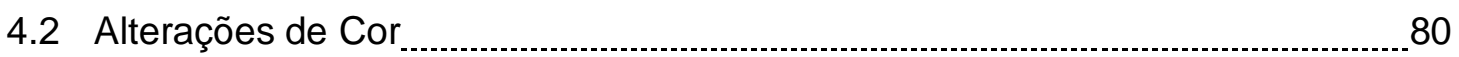

4.3 Alteração nas Coordenadas de Cor

4.4 Alteração de Translucidez 91

4.5 Linha de Cimentação

DISCUSSÃO

CONCLUSÃO

REFERÊNCIAS

APÊNDICE $\quad 127$ 

1. Introduçãa 



\section{INTRODUÇÃO}

Atualmente existe uma grande valorização da estética na sociedade, fazendo com que um grande número de pacientes procure os cirurgiões dentistas na busca de um sorriso mais bonito, um sorriso perfeito. Reabilitações estéticas são tratamentos que visam harmonizar o sorriso, através de restaurações protéticas. Segundo Radz, 2011 uma grande parte dessas restaurações são conhecidas como facetas laminadas, que são restaurações com espessura variando entre 0,5 a $0,7 \mathrm{~mm}$, cujo maior objetivo é devolver a estética do paciente.

A utilização de facetas laminadas em cerâmica consiste em uma das técnicas mais conservadoras utilizadas na Odontologia para restauração de dentes com comprometimento estético (Feire et al, 2010). Desde o seu desenvolvimento, há 30 anos, a correta interpretação de suas indicações e aplicações tem sido fundamental para propiciar adequado resultado clínico (Calamia, 1983; Gürel, 2007). Estudos em longo prazo (10 a 15 anos) demonstram que a taxa de sucesso em tratamentos com facetas laminadas é superior a 95\% (Friedman, 1998; Dumfahrt \& Schäffer, 2000). No entanto, existe dificuldade em avaliar a cor de laminados cerâmicos devido às numerosas variáveis envolvidas, como a cor da subestrutura do dente suporte (Stevenson \& Ibbetson, 2010), a espessura, a cor e o tipo de cerâmica (Della-Bona \& Anusavice, 2002, Dozic et al, 2003) e do cimento resinoso (Vichi et al, 2000;Lee et al, 2007; Chang et al, 2009), além da translucência do material restaurador evidenciado pela quantidade de reflexão e dispersão da luz (Seghiet al, 1986).

A cerâmica odontológica é o material de eleição para restaurações protéticas anteriores, pois apresenta excelentes propriedades, entre elas, estabilidade de cor (Milleding et al, 1998; Sjogreen et al, 1999; Pires-de-Souza et al, 2009), inércia química (Della Bona, 2005), translucidez (Bagis \& Turgut, 2012) e alta capacidade de mimetizar a estrutura dental (Pneumans et al, 2004; Braga et al, 2000), pois é o material restaurador que mais se assemelha as propriedades ópticas dos dentes (McLean, 1991; Wang et al, 2013). O potencial estético e a biocompatibilidade das cerâmicas podem ser considerados únicos entre os materiais restauradores dentais (Hooweg et al, 1998). 
Atualmente existe uma grande variedade de classes de cerâmicas disponíveis para distintas indicações, de acordo com seus fabricantes. Contudo não existe um único sistema cerâmico passível de ser empregado em todas as situações clínicas. Entretanto, a cerâmica prensada tem sido amplamente utilizada e indicada na confecção de laminados cerâmicos, uma vez que trata-se de uma técnica laboratorial fiel e previsível (Bahannan et al, 2016).

De acordo com Bagis \& Turgut, (2012) o método de obtenção da cerâmica não afeta a cor, croma e a translucidez, mas a diferente composição cristalina das cerâmicas pode determinar as propriedades ópticas da restauração.

Os sistemas cerâmicos são compostos por diferentes conteúdos cristalinos, que conferem resistência e por uma fase vítrea que confere cor e translucidez à cerâmica. A cerâmica a base de dissilicato de lítio tem boas propriedades mecânicas quando comparadas as cerâmicas convencionais, além de apresentar excelentes propriedades ópticas (Harada et al, 2016). No entanto, quando a cerâmica a base de dissilicato de lítio é comparada à cerâmica a base de zircônia, apresenta propriedades mecânicas inferiores, mas tem sido desempenho superior em termos de translucidez (Harada et al, 2016). Entretanto, tem sido desenvolvidas cerâmicas a base de zircônia com alto grau de translucidez (Manual e. max Zirpress, 2009), com intuito de conferir maior estética a restaurações monolíticas. No entanto, existem poucos dados na literatura da translucidez da cerâmica a base de zircônia em comparação com o material a base de dissilicato de lítio.

Apesar das excelentes propriedades estéticas das cerâmicas, uma das principais dificuldades em reconstruções estéticas com estes materiais é mimetizar a estrutura dental (Dozic et al, 2010). O dente interage com a luz de forma complexa, pois é constituído de diferentes estruturas e tecidos, em diferentes espessuras, que resulta num policromatismo. Por isso é tão difícil obter naturalidade nas restaurações a partir dos materiais que são monocromáticas (Lambrechts et al, 1990).

A longevidade da restauração é um fator muito importante no tratamento e está relacionado com a estabilidade de cor do material (Miyagawa et al,1981; Heffernan et al, 2002; Lu \& Powers, 2004, Turgut \& Bagis, 2011), uma vez que trata-se de um tratamento 
estético. Sendo assim, a estabilidade de cor é um fator adicional a ser enfrentado pelos cirurgiões dentistas que buscam sucesso e longevidade nos tratamentos realizados por eles.

Um desafio importante na estética é combinar as propriedades ópticas dos dentes naturais com restaurações (Tung et al, 2002). Clinicamente, uma combinação de cores adequada é aspecto importante em qualquer restauração dentária estética (Meijering et al, 1997). A combinação final de cores de restaurações protéticas de cerâmica em relação à dentição natural adjacente continua problemática, principalmente em facetas laminadas (Haselton et al, 2000; Gurel, 2007; Corciolani et al, 2010; Dozic et al, 2010).

Vários fatores estão envolvidos na determinação da cor de um objeto, como as características da fonte de luz sob a qual o objeto é observado; a forma como as ondas de luz incidentes são absorvidas, transmitidas ou refletidas e os efeitos do ambiente sobre o observador (Schanda, 1998; Janda et al, 2004).

A cor não é simplesmente uma propriedade da luz, mas uma resultante do efeito das ondas de luz refletida pelos materiais restauradores, podendo estes ser opacos ou translúcidos (Jonhston \& Reisbick, 1997). Associado a cor, a translucidez afeta o resultado estético das restaurações, pois é essa propriedade que confere a naturalidade à restauração (Crisp et al, 1979; Kelly et al,1996; Sorensen et al, 1999, Bagis \& Turgut, 2012). Clinicamente, quando restaurações translucentes são cimentadas sobre dentes sem alteração de cor, isso pode resultar em uma boa recuperação estética (Bargh i\& McAlister, 1998).

A translucidez ou a opacidade de um material depende da absorção e do espalhamento da luz no seu interior (Zahng et al, 2004, Lee et al, 2005 ). Dessa forma, se a maior parte da luz que passa através da cerâmica é intensamente espalhada ou refletida de forma difusa, o material terá aparência opaca, se parte da luz for espalhada e a maioria é transmitida, o material parecerá translúcido (Heffernan et al, 2002, Lee et al, 2005). As quantidades de luz absorvida, refletida e transmitida são determinadas pelo comportamento óptico do material e dependem das características microestruturais, morfológicas do material e da luz incidente (Heffernan et al, 2002, Zahng et al, 2004, Lee et al, 2005, Luo \& Zhang, 
2010). Portanto, conhecer a interação da luz com os objetos auxilia o cirurgião-dentista a selecionar a cor dos materiais restauradores de forma adequada para realização de um procedimento reabilitador onde a estética é fundamental (Schanda, 1998).

O sucesso clínico de um tratamento reabilitador protético depende em parte da técnica de cimentação utilizada (Radovic et al, 2011). Cimentos resinosos são indicados para fixação das facetas laminadas, uma vez que os preparos para essas restaurações são muito conservadores, com pouca ou nenhuma retenção (Magne et al,1999) e uma cimentação adesiva se faz necessária.

Os cimentos resinosos fotopolimerizáveis são os materiais de primeira escolha para cimentação de laminado cerâmico devido á possibilidade de o cirurgião dentista conseguir controlar o tempo do procedimento, uma vez que a polimerização está condicionada à exposição à luz (Radz, 2011). No entanto, a cimentação com esse tipo de cimento constitui um procedimento clínico de grande complexidade, incluindo muitos passos, dificultando a técnica, podendo levar a falhas e erros durante tal procedimento (De Munck et al, 2004).

Os cimentos autoadesivos surgiram no mercado com a proposta de simplificar a técnica adesiva, com a aplicação do cimento em um único passo, eliminando o tratamento prévio do substrato dentário, diminuindo assim a sensibilidade da técnica adesiva (Han, 2007; Cantoro et al, 2010; Pavan et al ,2010; Nakamura et al, 2010). Este cimento possui na sua composição monômeros acidificados mais potentes que os tradicionais, proporcionando adesão à estrutura dentinária, não sendo necessário realizar o preparo do substrato, previamente à cimentação, diminuindo dessa forma o número de passos clínicos (Sazer et al, 2014). Esses cimentos apresentam resistência de união comparada ao do cimento resinoso convencional (Walter et al, 2005).

Os cimentos fotoativados e dual possuem sistema de ativação diferentes, podendo diferir no grau de conversão dos monômeros, e ao longo do tempo no resultado final da restauração protética, referindo-se a estabilidade de cor e translucidez de laminados cerâmicos, que ainda continuam indefinidas. 
Outro fator a ser considerado é a espessura da película do cimento que, segundo Andrade et al (2000), é uma característica inerente de cada cimento e está relacionado ao tamanho e a forma das partículas, consistência e grau de polimerização. A linha de cimentação não deve ultrapassar $100 \mu \mathrm{m}$, pois uma desadaptação maior resultaria em desgaste excessivo do agente cimentante, dando origem a fraturas das margens, infiltrações e cáries secundárias (Leinfelder et al, 1989). Adabo et al (2001), encontraram valores de película de diversos cimentos resinosos muito próximos aos preconizados pela ADA (American Dental Association) e para o cimento fosfato de zinco na ordem de $25 \mu \mathrm{m}$.

A cor do cimento resinoso pode produzir alterações de cor significantes na restauração final com cerâmicas (Change et al, 2009; Xing et al, 2010), principalmente em facetas laminadas, devido sua fina espessura. Por outro lado, há estudos (Vichi et al, 2000; Azer et al, 2006) que reportam que não há influência significativa dos cimentos resinosos na cor final de materiais totalmente cerâmicos. Não há consenso na literatura sobre a influência da cor do cimento na cor final da restauração de porcelana.

Embora a translucidez das cerâmicas e a alteração de cor de cimentos resinosos têm sido avaliadas na literatura, a interação de laminados cerâmicos, cimento resinoso e estrutura dental ainda precisam ser esclarecidos, devido a um complexo comportamento óptico advindo da junção de diferentes estruturas. Dessa maneira, o objetivo do presente estudo será avaliar o grau de conversão dos cimentos resinosos quando polimerizados através de diferentes espessuras de laminados cerâmicos, a linha de cimentação produzida por cada cimento e a influência desses cimentos e dos sistemas cerâmicos na estabilidade de cor e na translucidez de facetas laminadas. 

2. Propasicãa 



\section{ProposiçÃo}

O objetivo desse estudo in vitro foi avaliar o grau de conversão, a linha de cimentação e a estabilidade de cor de cimentos resinosos submetidos a diferentes protocolos de envelhecimento e verificar a influência desses fatores na translucidez de laminados cerâmicos em diferentes espessuras.

As hipóteses testadas foram:

$\checkmark$ Não haverá diferença no grau de conversão dos cimentos em função das espessuras das restaurações

$\checkmark$ Não haverá diferença na estabilidade de cor e translucidez das restaurações em função do agente cimentante e do protocolo de envelhecimento a que foi submetido.

$\checkmark$ Restaurações mais delgadas sofrerão maior influência na alteração de cor e trnslucidez 

3. Materiais e Método 



\section{MATERIAIS E MÉTODO}

Para a realização deste estudo foram utilizados os materiais descritos na Tabela 1.

Tabela 1- Materiais utilizados no estudo.

\begin{tabular}{|c|c|c|}
\hline Nome Comercial & Composição & Fabricante \\
\hline $\begin{array}{l}\text { IPS e-maxPress } \\
\text { Cor A1 HT }\end{array}$ & $\begin{array}{l}\text { Componentes: } \mathrm{SiO}_{2} \\
\text { Componentes adicionais: } \mathrm{Li}_{2} \mathrm{O}, \mathrm{K}_{2} \mathrm{O}, \mathrm{MgO} \\
\mathrm{ZnO}, \mathrm{Al}_{2} \mathrm{O}_{3}, \mathrm{P}_{2} \mathrm{O}_{5} \text { e outros óxidos. }\end{array}$ & $\begin{array}{l}\text { Ivoclar/Vivadent AG, } \\
\text { Schaan, Liechtenstein }\end{array}$ \\
\hline $\begin{array}{l}\text { IPS e-maxZirpress } \\
\text { Cor A1 HT }\end{array}$ & $\begin{array}{c}\text { Componentes: } \mathrm{SiO}_{2} \\
\text { Componentes adicionais: } \mathrm{Li}_{2} \mathrm{O}, \mathrm{Na}_{2} \mathrm{O}, \mathrm{K}_{2} \mathrm{O} \text {, } \\
\mathrm{MgO}, \mathrm{Al}_{2} \mathrm{O}_{3}, \mathrm{CaO}, \mathrm{ZrO}_{2}, \mathrm{P}_{2} \mathrm{O}_{5} \mathrm{e} \text { outros óxidos }\end{array}$ & $\begin{array}{l}\text { Ivoclar/Vivadent AG, } \\
\text { Schaan, Liechtenstein }\end{array}$ \\
\hline $\begin{array}{l}\text { Rely-X U200 } \\
\text { Transparente }\end{array}$ & $\begin{array}{l}\text { Pasta base: fibra de vidro, ésteres ácido } \\
\text { fosfórico metacrilato, TEGDMA, sílica } \\
\text { tratada com silano e persulfato de sódio. } \\
\text { Pasta catalisadora: fibra de vidro, } \\
\text { dimetacrilato substitutivo, sílica tratada com } \\
\text { silano, P-toluenosulfonato de sódio e } \\
\text { hidróxido de cálcio. }\end{array}$ & $\begin{array}{l}\text { 3M ESPE, Seefeld, } \\
\text { Alemanha }\end{array}$ \\
\hline $\begin{array}{l}\text { Variolink II } \\
\text { Transparente }\end{array}$ & $\begin{array}{c}\text { Monômeros: Bis-GMA, UDMA, EDMA } \\
\text { Partículas de carga: sílica, vidro de bário, } \\
\text { trifluoreto de itérbio } \\
\text { Outros componentes: catalizadores, } \\
\text { estabilizadores e pigmentos }\end{array}$ & $\begin{array}{l}\text { Ivoclar/Vivadent AG, } \\
\text { Schaan, Liechtenstein }\end{array}$ \\
\hline
\end{tabular}

\subsection{Preparo dos dentes}

Para a realização deste trabalho, foram utilizados 270 incisivos bovinos recémextraídos, mantidos em solução de timol $0,1 \%$ à temperatura de $4^{\circ} \mathrm{C}$, os quais foram limpos com o auxílio de pedra pomes e água com escovas de Robinson. Dentes com manchas, trincas ou anomalias de estrutura foram excluídos do estudo.

Os dentes tiveram suas coroas e raízes separadas por meio de uma secção transversal localizada a $1 \mathrm{~mm}$ abaixo da junção amelocementária com disco diamantado flexível montado em cortadeira metalográfica de precisão (Isomet 1000, Norfolk, Massachusetts, USA).

A porção coronária dos dentes foi incluída em anel de PVC com resina acrílica (Vipi Flash, Vipi, Pirassununga, SP, Brasil, Figura 1). Em seguida, os dentes tiveram a superfície vestibular planificada até dentina, em único corte, com disco diamantado flexível montado em cortadeira metalográfica de precisão (Isomet 1000, Buehler). O disco foi posicionado em contato com a bossa vestibular e em seguida recuou-se $2 \mathrm{~mm}$ para realização e 
padronização do corte em todos os dentes. Após o aplainamento (Figura 2), os dentes foram armazenados em água destilada em estufa a $37^{\circ} \mathrm{C}$.

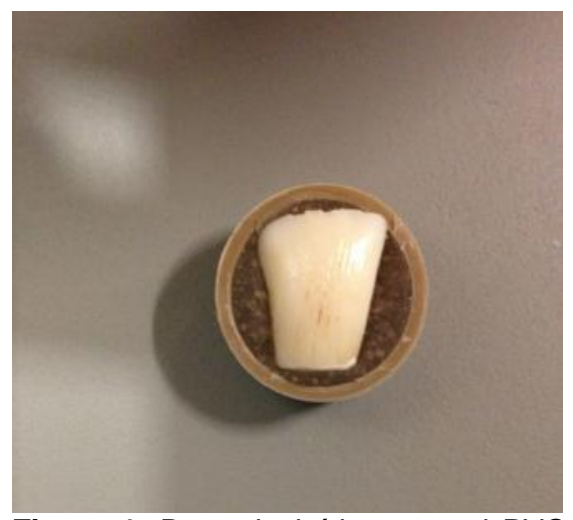

Figura 1- Dente incluído em anel PVC com resina acrílica.

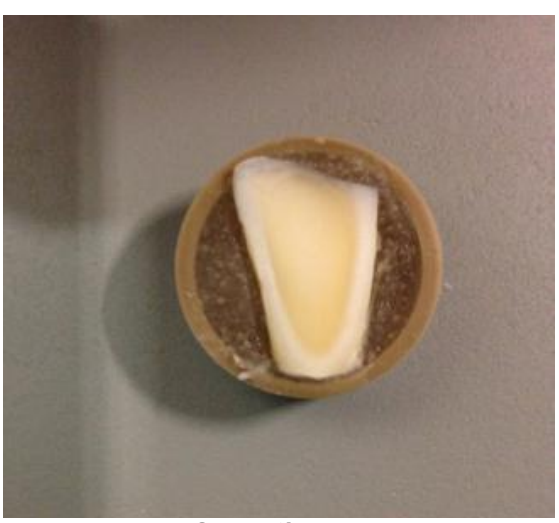

Figura 2- Superfície vestibular do dente cortada em dentina.

\subsection{Confecção dos corpos-de-prova}

A confecção dos laminados cerâmicos em duas espessuras $(0,5$ e 1,0mm) e $6 \mathrm{~mm}$ de diâmetro, foi feita pela técnica de enceramento e prensagem da cerâmica. Para isso, foi utilizada matriz de teflon com mesmas dimensões dos corpos-de-prova. A matriz de teflon é formada por duas partes: uma externa e uma interna, contendo um êmbolo, de $6 \mathrm{~mm}$ de diâmetro. Conforme a espessura do laminado cerâmico a ser obtido, foi utilizado um espaçador de mesma espessura, encaixado no êmbolo, e disposto entre as porções superior e inferior da matriz.

O enceramento dos corpos-de-prova foi feito com cera orgânica (Rainbow, FABRICANTE, Porto Ferreira, SP, Brasil), própria para enceramentos de peças a serem trabalhadas com cerâmica prensada, uma vez que, para se trabalhar com essa técnica fazse necessário que a cera seja eliminada completamente, não restando nenhum resíduo, para que não haja contaminação da cerâmica e, consequentemente, manchamento por pigmentos provenientes da não eliminação total da cera (Manual técnico IPS e-max Press, 2009). Assim, a cera foi derretida, com auxílio de uma base de metal e lamparina, e gotejada sobre a matriz de teflon previamente vaselinada (Figura 3). 


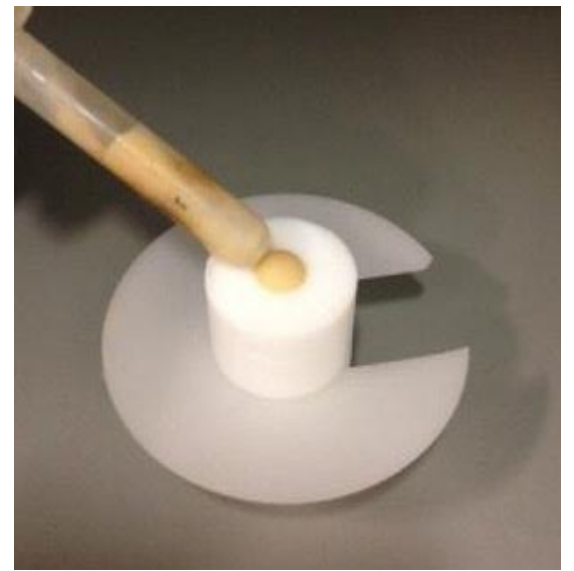

Figura 3- Gotejamento de cera em matriz para confecção dos corpos-deprova.

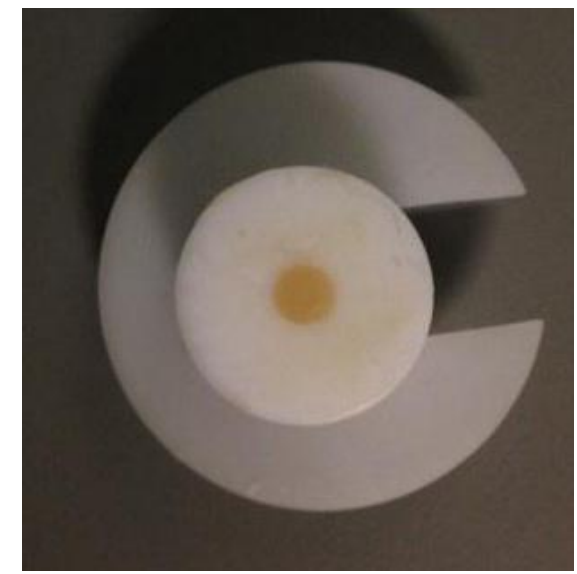

Figura 4- Solidificação dos padrões de cera.

Após a solidificação da cera (Figura 4), os padrões foram removidos e incluídos na base do anel de silicone (IPS silicone Ring, Ivoclar/Vivadent AG Schaan, Liechtenstein) com auxílio da cera orgânica (Rainbow, Porto Ferreira, SP, Brasil) e sprues confeccionados com fios de cera de $2.5 \mathrm{~mm}$ de diâmetro e $3 \mathrm{~mm}$ de comprimento. Os sprues foram posicionados sobre os corpos-de-prova com inclinação entre 45 a 60 graus (Figura 5), na direção de escoamento dos materiais cerâmicos e em seguida o cilindro do anel de silicone foi colocado sobre a base, deixando um espaço de $10 \mathrm{~mm}$ entre o corpo-de-prova e o cilindro, ocupando no máximo uma altura de $16 \mathrm{~mm}$, para deixar um espaço de $10 \mathrm{~mm}$ entre os corpos-de-prova e a abertura do anel.

O espaço entre um sprue e outro deve ser de $3 \mathrm{~mm}$. Sendo assim foram colocados 10 corpos-de-prova por anel (Figura 6). Todo o conjunto recebeu o borrifamento do spray redutor de tensão superficial (Lubrofilm, Dentaurum, Ispringen, Deutschlat). Depois de finalizado o posicionamento dos corpos-de-prova dentro do anel de silicone, o revestimento (IPS PressVEST, Ivoclar/Vivadent AG Schaan, Liechtenstein) foi dosado e manipulado seguindo as orientações do fabricante, vertido dentro do anel e aguardou-se a presa do revestimento. 


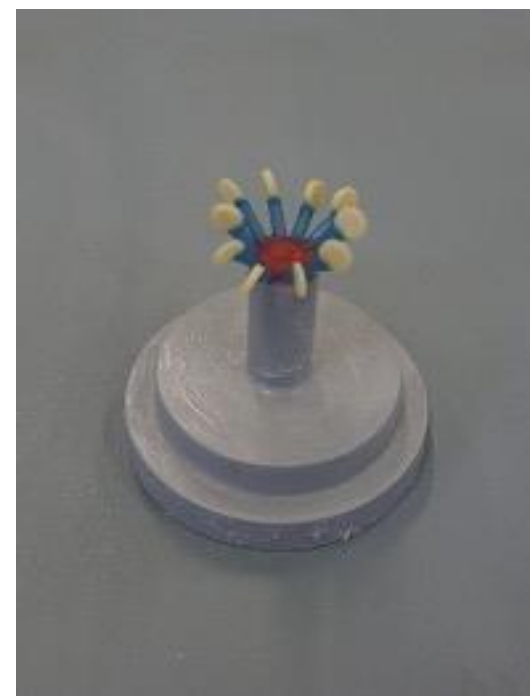

Figura 5- Padrões de Cera posicionados a $45^{\circ}$.

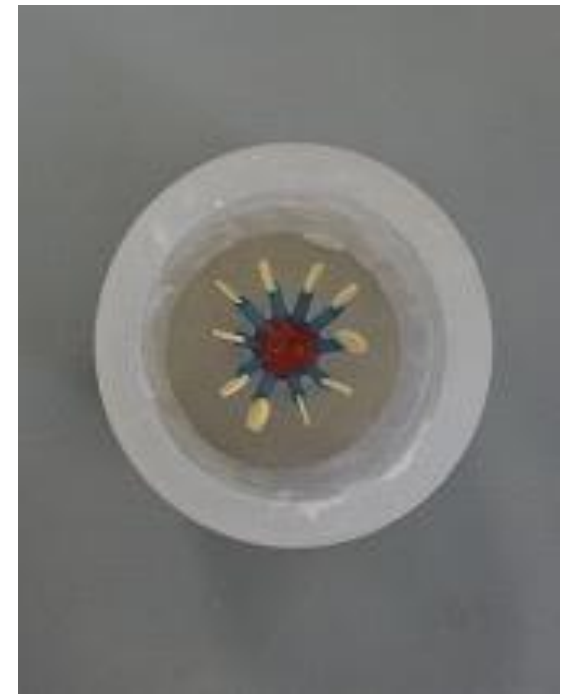

Figura 6- Padrões de cera em anel de silicone para revestimento.

Após a presa do revestimento, foi feita a remoção do anel de silicone e o revestimento foi levado ao forno (Forno EDG 3000-3P, EDG Equipamentos e Controles, São Carlos, SP, Brasil, Figura 7) para remoção da cera. A temperatura aumentou $5^{\circ} \mathrm{C}$ por minuto até alcançar $850^{\circ} \mathrm{C}$. Finalizada a remoção da cera, o revestimento foi levado imediatamente para o forno (Programat EP 5000 Ivoclar/Vivadent AG Schaan, Liechtenstein) de injeção e prensagem da cerâmica, para que não ocorresse o resfriamento do revestimento, com auxílio de uma pinça para manuseio do revestimento (Figura 8). O bloco de cerâmica frio foi posicionado sobre o revestimento quente e foi pressionado para dentro do orifício do revestimento com auxílio de um êmbolo (Aloxlvoclar/Vivadent AG Schaan, Liechtenstein) de alumina. O êmbolo foi impregnado previamente com um pó de nitrato de boro (IPS Alox Plunger Separator Ivoclar/Vivadent AG Schaan, Liechtenstein) para facilitar a prensagem da cerâmica e não permitir que a cerâmica ficasse aderida ao êmbolo.

O processo de injeção e prensagem dos blocos de cerâmica foi padronizado para cada sistema cerâmico, de acordo com a programação específica contida no software V3.3 (Manual técnico IPS e-max Press, 2009 e Manual técnico IPS e-max ZirPress, 2009). 


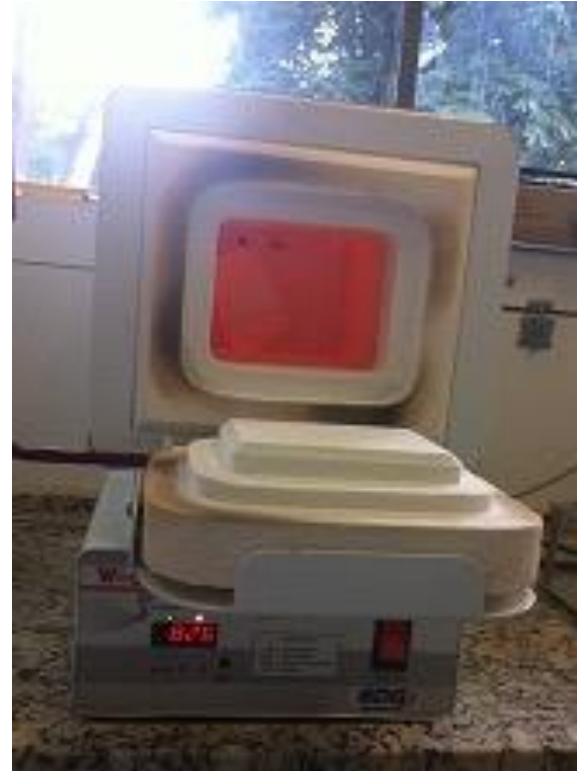

Figura 7- Revestimento posicionado para eliminação da cera.

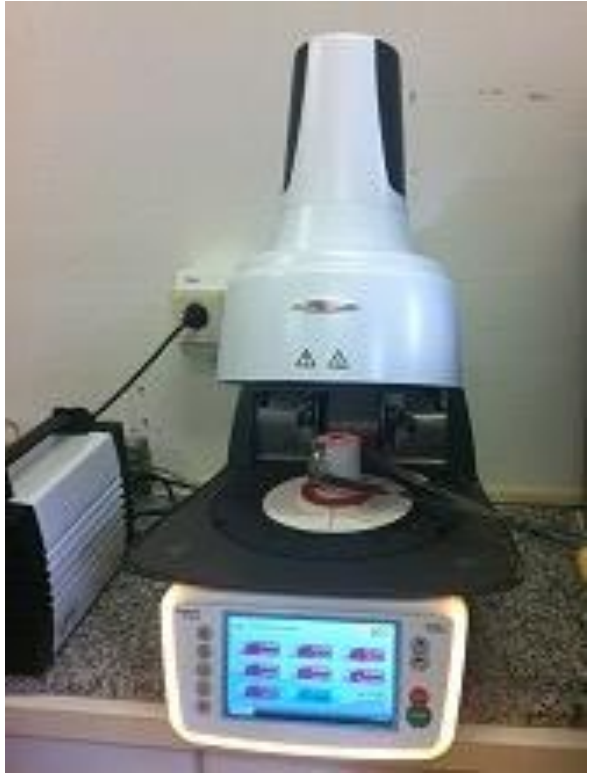

Figura 8- Injeção de cerâmica.

Foram obtidos 120 corpos-de-prova de cada cerâmica, sendo 60 corpos-de-prova na espessura de $0,5 \mathrm{~mm}$ e outros 60 na espessura de $1,0 \mathrm{~mm}$. Após o resfriamento até a temperatura ambiente (Figura 9), os corpos-de-prova foram removidos do anel de revestimento (Figura 10). O acabamento foi realizado com jateamento e microesferas de vidro para polimento (Renfert, Hilzingen, Alemanha) com 4 bar (60 psi) de pressão; para a remoção final do revestimento foi empregado somente 2 bar (30psi). Os condutos de

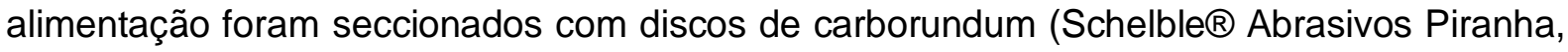
Petrópolis, RJ, Brasil) e o acabamento e polimento dos corpos-de-prova foram realizados com pedras abrasivas, brocas diamantadas e pontas de borracha específicas (EVE Ernst VetterGmbH, Pforzheim, Alemanha). 


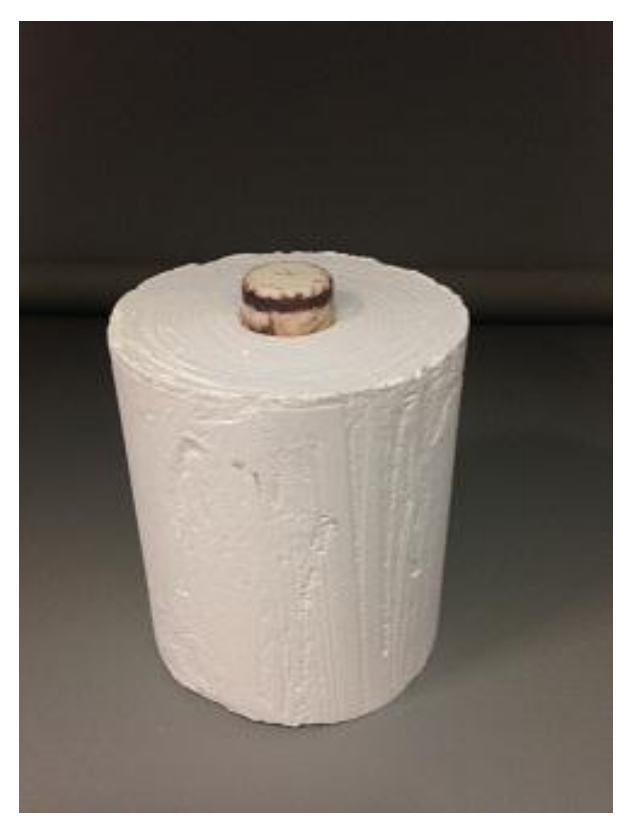

Figura 9- Revestimento resfriado após injeção da cerâmica.

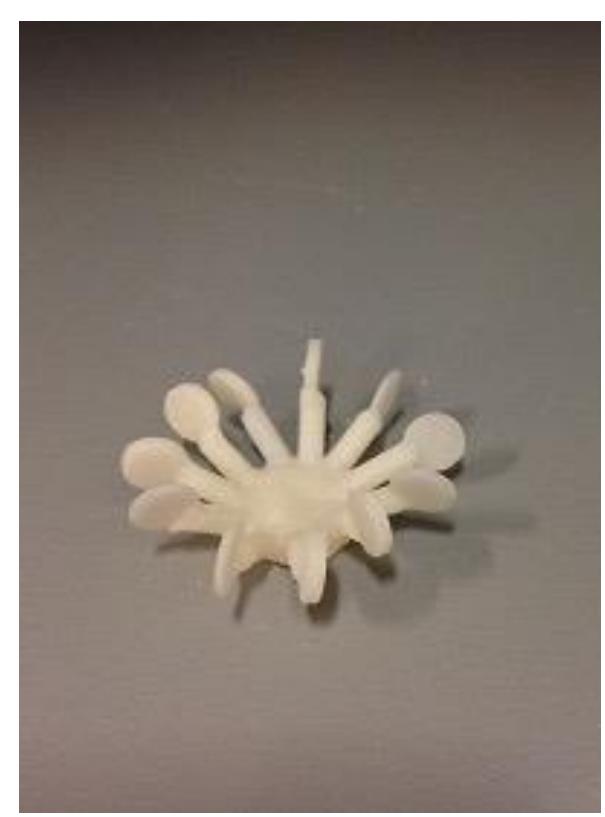

Figura 10- Corpos-de-prova de cerâmica após desinclusão.

Em seguida, os corpos-de-prova foram imersos em cuba plástica, no ultrassom (Cristófoli Equipamentos de Biossegurança LTDA, Campo Mourão, Paraná, Brasil) por 10 minutos utilizando IPS Invex Liquid (Ivoclar/Vivadent AG Schaan, Liechtenstein) para a remoção da camada da reação que é formada durante processo de injeção. Depois os corpos-de-prova foram lavados em água corrente, secos com jato de ar e jateados com $\mathrm{Al}_{2} \mathrm{O}_{3}$ (tipo 100) com 1-2 bar (15-30 psi) de pressão para remover a camada branca da reação que ocorre durante a limpeza das peças.

Os corpos-de-prova receberam polimento com lixas d'água (Norton) em ordem decrescente de granulação (600, 1000 e 1200) em politriz mecânica (Polipan-U, Panambra, São Paulo, SP, Brasil), e tiveram suas espessuras aferidas com paquímetro digital (Digimess Instrumentos de Precisão LTDA, São Paulo, SP, Brasil, Figura 11 e 12). 


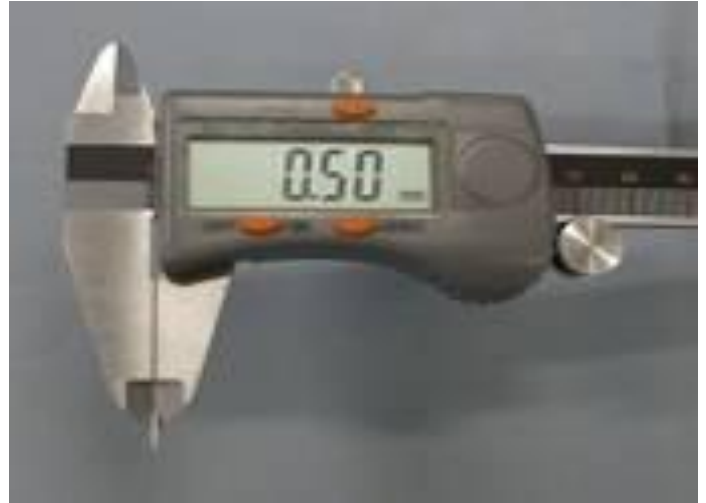

Figura 11- Mensuração de 0,5mm.

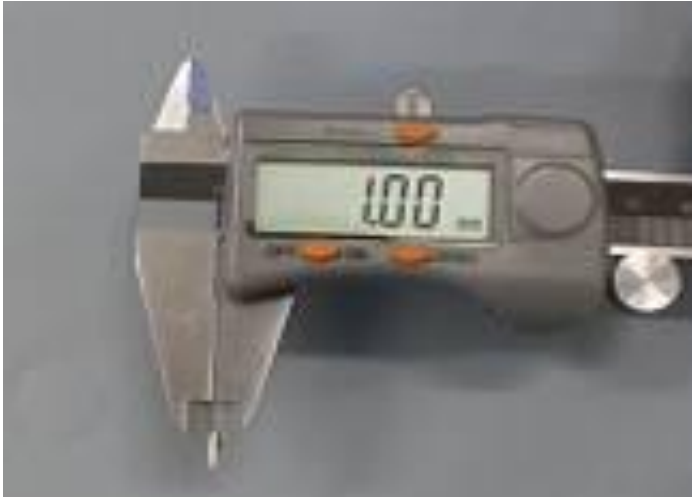

Figura12- Mensuração de 1,0mm.

O glazeamento foi realizado no mesmo forno de prensagem à temperatura de $403^{\circ} \mathrm{C}$ por 6 minutos e $770^{\circ} \mathrm{C}$ por 1,5 minutos para ambas cerâmicas (IPS e. max Press e IPS e. maxZirpress, Figura 13), seguindo as recomendações do fabricante.

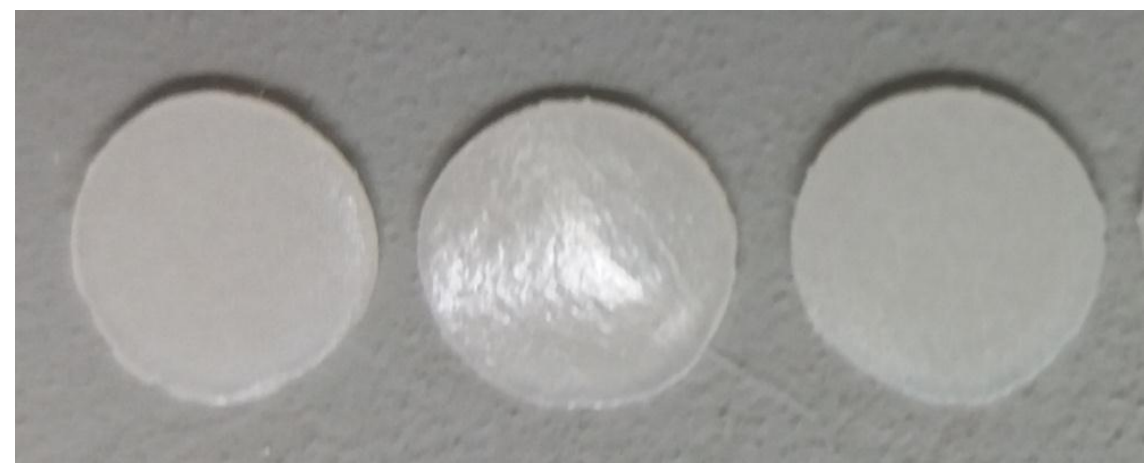

Figura 13- Imagem dos corpos-de-prova finalizados, com glaze.

\subsection{Cimentação dos corpos-de-prova}

Os corpos-de-prova de cada cerâmica foram aleatoriamente separados em grupos de 30 amostras de cada espessura para serem fixados nos dentes, utilizando 2 tipos de cimentos resinosos: um cimento fotoativado (pasta base do Variolink II, Ivoclar/Vivadent AG Schaan, Liechtenstein) e um autoadesivo (Rely-X U200, 3M do Brasil, Sumaré, São Paulo, Brasil).

Antes da cimentação, todos os corpos-de-prova foram limpos com álcool 90\%, em ultrassom (Cristófoli, Cristófoli Equipamentos de Biossegurança) por 4 minutos e em seguida receberam o tratamento da peça. Para a cerâmica IPS e.maxPress o tratamento consiste no condicionamento com ácido fluorídrico a 10\% (Condicionador de Porcelanas, Dentsply, 
Petrópolis, RJ, Brasil, Figura 14) por 20 segundos, lavagem, secagem e aplicação do silano (Monobond S, Ivoclar/Vivadent, Figura 15) por 1 minuto. Para a cerâmica IPS e.maxZirpress o tratamento da peça consiste no jateamento com óxido de alumínio (Figura 16) com pressão de 1 bar (15 psi) e aplicação de silano (Figura 17) por 60 segundos.

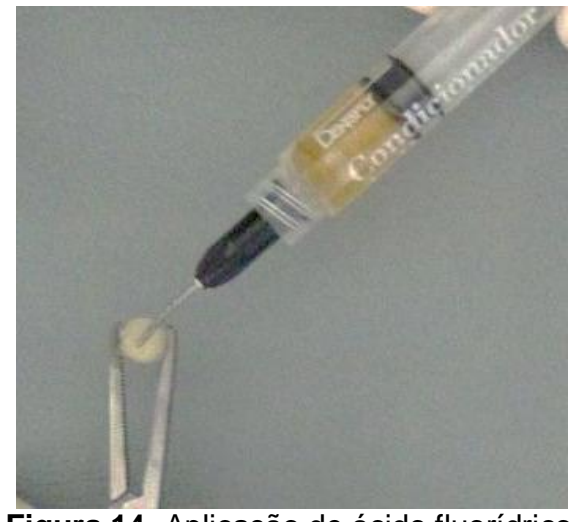

Figura 14- Aplicação de ácido fluorídrico.

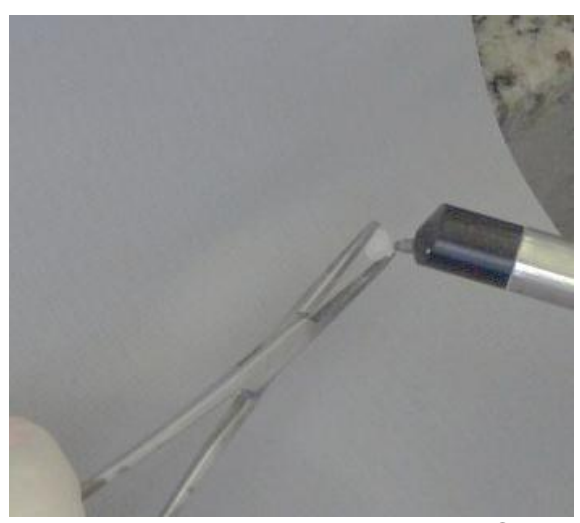

Figura 16- Jateamento com $\mathrm{Al}_{2} \mathrm{O}_{3}$.

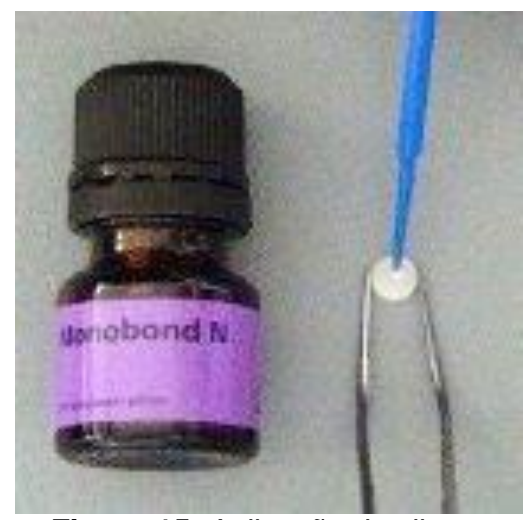

Figura 15- Aplicação de silano.

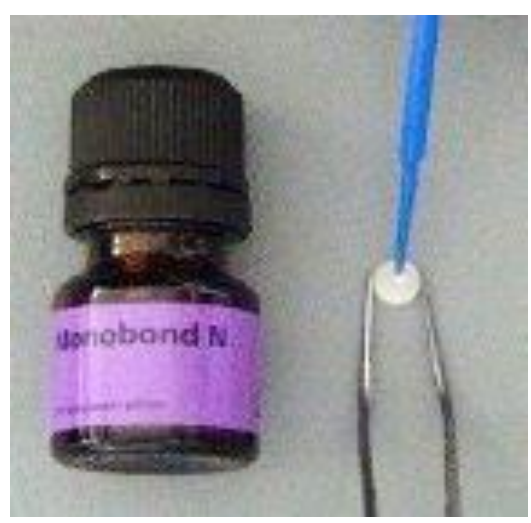

Figura 17- Aplicação de silano.

Os dentes tiveram a superfície vestibular polida em politriz mecânica (Polipan-U, Panambra, São Paulo, SP, Brasil) com lixas d'agua abrasivas (Norton, São Paulo, SP, Brasil) em granulação \#600 para formação da smear layer.

Para cimentação com cimento autoadesivo (Transparente, Figura 18), não é necessário o tratamento do substrato. Então após o preparo da peça, foram dispensadas (Figura 19) e manipuladas partes iguais da pasta base e catalisadora durante 10 segundos, conforme orientação do fabricante. Em seguida, o cimento foi aplicado sobre no centro do corpo-de-prova com uma espátula n 1 e o conjunto foi posicionado sobre a face vestibular do dente aplainado. O excesso de cimento foi removido com sonda exploradora $n \div 5$ e 
realizada fotoativação por 40 segundos (FLASHlite 1401, Discus Dental, Culver City, CA, EUA).

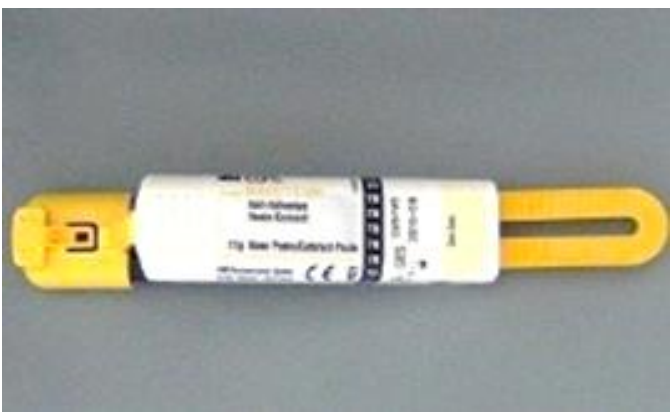

Figura 18- Cimento autoadesivo.

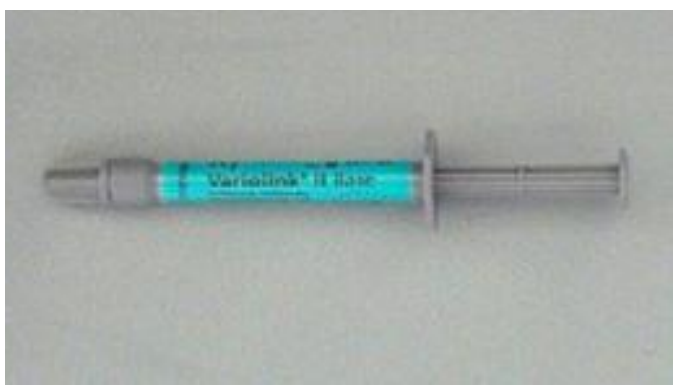

Figura 20- Cimento fotopolimerizável.

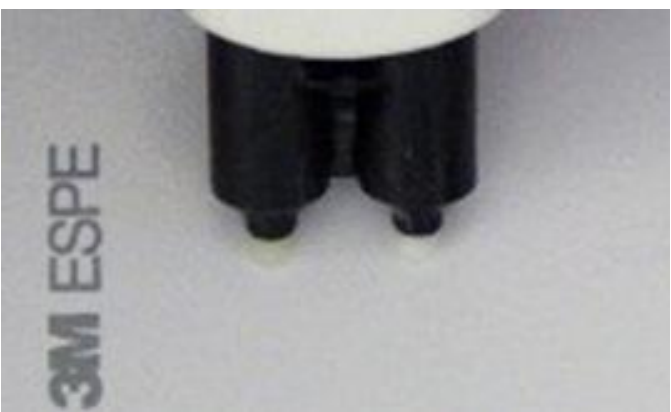

Figura 19- Dosagem do cimento.

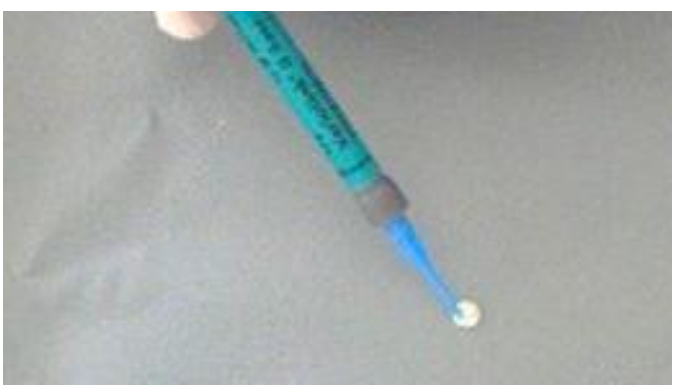

Figura 21- Aplicação do cimento.

Quando utilizado um cimento fotoativado (Transparente, Figura 20), o preparo do substrato foi previamente realizado e consistiu em condicionamento ácido do dente com ácido fosfórico a 37\% (Figura 22) por 15 segundos, lavagem com água (Figura 23) e remoção do excesso de umidade com papel absorvente, aplicação do sistema adesivo (Tetric N Bond, Ivoclar/Vivadent AG Schaan, Liechtenstein, Figura 24) sobre o dente, em seguida um leve jato de ar e fotoativação (FLASHlite 1401, Discus Dental) do adesivo por 20 segundos.

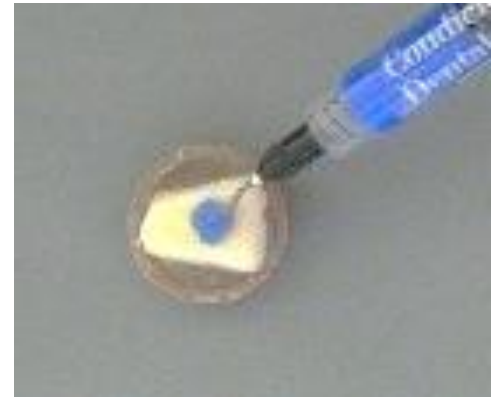

Figura 22- Aplicação ácido fosfórico.

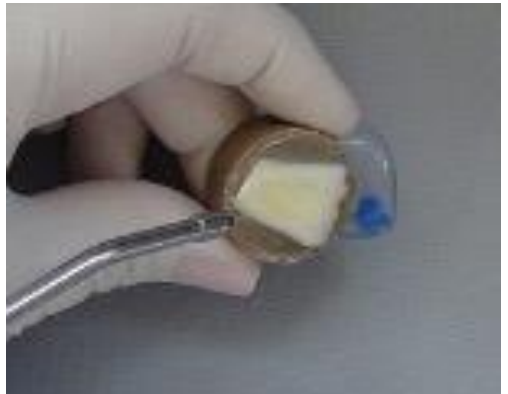

Figura 23- Lavagem.

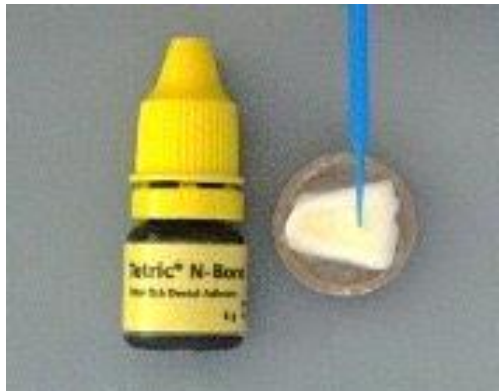

Figura 24- Aplicação do adesivo. 
Após o tratamento do substrato a pasta base do cimento foi aplicada sobre o centro do corpo-de-prova com auxílio de uma espátula no 1 e o conjunto foi assentado na face vestibular preparada do dente, conforme descrito anteriormente.

Os corpos-de-prova foram fixados nos dentes seguindo padrões de paralelismo e carga (Figuras 25 e 26). Após o posicionamento de corpo-de-prova sobre o dente foi utilizada a haste de um delineador para garantir o paralelismo na fixação da peça. Uma carga de 380 gramas foi utilizada por 5 segundos para garantir a padronização da força aplicada no procedimento de cimentação. O excesso foi delicadamente removido com sonda exploradora ํ5 e a fotoativação realizada com aparelho tipo LED (FLASHlite 1401, Discus Dental, Figura 27) por 40 segundos. Após cimentação, as amostras foram mantidas em água destilada por 24 horas a $37^{\circ} \mathrm{C}$ e em seguida, foram realizadas as primeiras leituras de cor e translucidez.

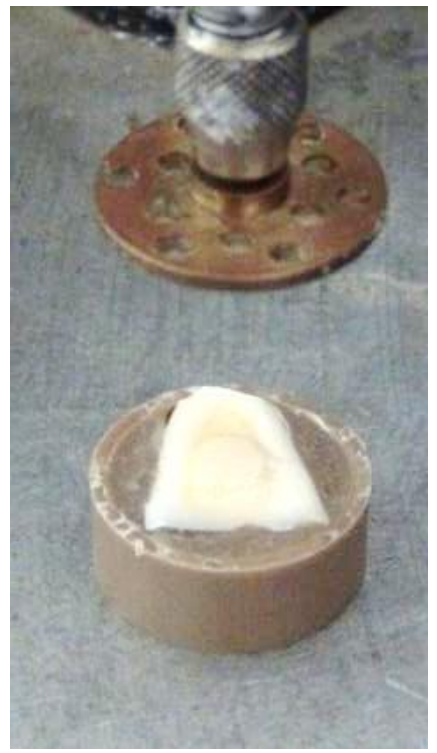

Figura 25- Paralelismo.

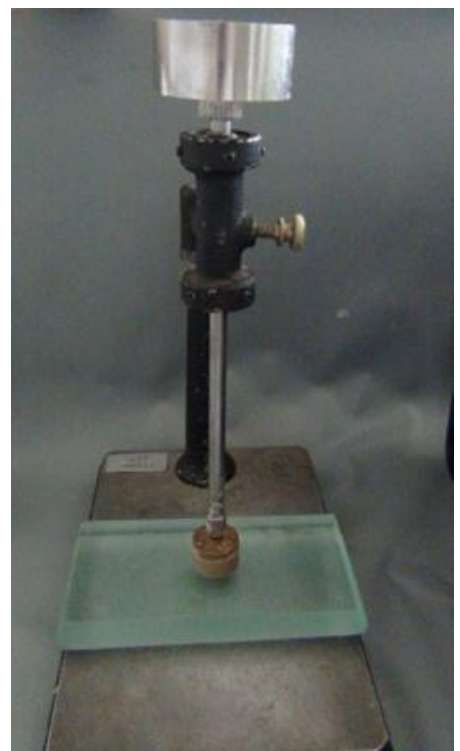

Figura 26- Delineador e peso.

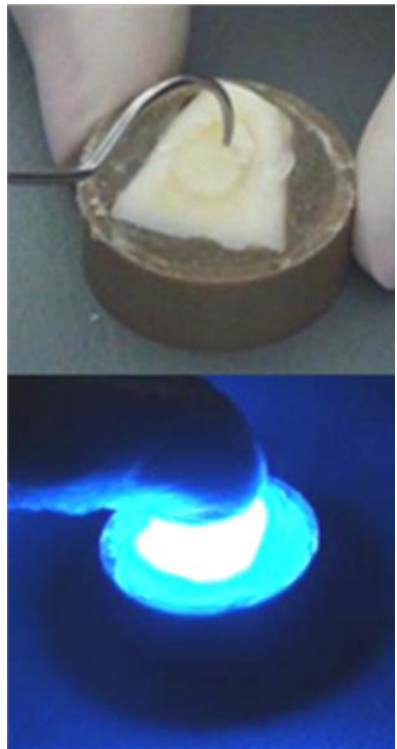

Figura 27- Remoção do excesso e fotoativação do cimento.

Também foram obtidos dois grupos-controle: um relacionado aos cimentos e outro aos dentes. Assim, foram confeccionados 10 corpos de prova de cada cimento, utilizando a mesma matriz descrita anteriormente, em uma só espessura $(0,5 \mathrm{~mm})$. Para isso, as pastas base e catalisadora do cimento autoadesivo foram dispensadas em comprimentos iguais e 
manipuladas da mesma maneira quando o cimento foi utilizado para fixação das peças, inserido na matriz e fotopolimerizado por 40 segundos. O cimento fotoativado, que é constituído apenas por uma pasta, foi inserido diretamente na mesma matriz e fotopolimerizado por 40 segundos.

Trinta dentes $(n=10)$, dos 270 selecionados, foram utilizados como grupo controle. Para isso, foram desgastados até dentina sobre os quais não foi fixado nenhum corpo de prova ou cimento. Tanto os dentes como as amostras de cimento após a obtenção, foram mantidos em saliva artificial a $37^{\circ} \mathrm{C}$ por $24 \mathrm{~h}$ e em seguida, foram realizadas as primeiras leituras de cor e translucidez.

\subsection{Leituras de cor e translucidez}

Para as leituras de cor e translucidez foi utilizado o equipamento Vita Easyshade (VITA Zahnfabrik, BadSäckingen, Alemanha, Figura 28), que possui uma ponteira digital com $6 \mathrm{~mm}$ de diâmetro, 19 fibras ópticas individuais que iluminam o material restaurador e 2 sensores espectrofotométricos capazes de ler numericamente a cor. O padrão de observação simulado pelo colorímetro Espectrofotométrico segue o sistema CIE $L^{*} a^{*} b^{*}$, recomendado pela CIE (Comission Internationale de l'Éclairage) e amplamente usado atualmente (Silami et al, 2016).

A geometria óptica de medição de cor é circular com o componente especular excluído, o que simula uma medição 45/0. O iluminante padrão é o D65 e o observador padrão de 2․ O componente especular excluído é relacionado à medição da cor na superfície da amostra, para evitar a interferência do brilho da superfície (Devigus et al., 2004a; Devigus et al., 2004b).

O Sistema de determinação de cor CIE Lab (Commission Internationale l'Éclairage, 1976) é definido por uma combinação de coordenadas cartesianas onde um ponto está associado a uma cor única. O sistema de coordenadas é composto pelo eixo $L^{*}$ coordenada de luminosidade (escala de cinza), cujos valores numéricos variam de 0 (preto) a 100 (branco). Quanto mais próximo de zero, mais escura é a cor do objeto. Os eixos a* e 
b*, são conhecidos como coordenadas de cromaticidade e variam de -128 a +128 . A coordenada $a^{*}$ designa o eixo vermelho - verde. Quando o valor para $a^{*}$ for positivo (+) mostrará desvio no sentido da tonalidade vermelha, quando o valor for negativo (-) mostrará desvio no sentido do verde. Para a coordenada $b^{*}$, os valores positivos são fornecidos para os objetos da tonalidade amarela e negativos para a tonalidade azul (Wyszecki \& Stiles, 1982).

Para o ensaio de estabilidade de cor, as mensurações da cor das amostras foram realizadas utilizando um fundo padrão branco (Mast Soluções de Qualidade, Santo André, SP, Brasil, Figura 29 e 30) e para o ensaio de translucidez, as amostras foram posicionadas sobre fundos padrão branco e preto (Mast Soluções de Qualidade, Santo André, SP, Brasil)

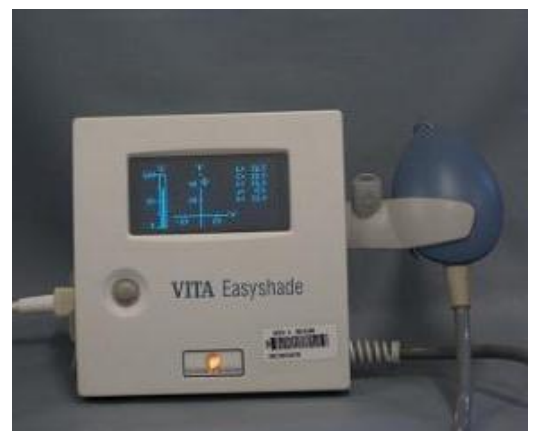

Figura 28- Vita EasyShade.

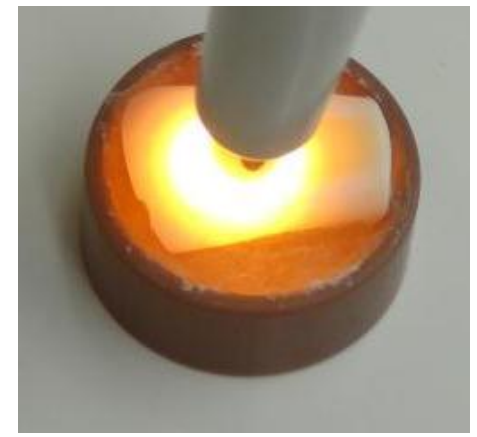
$\begin{array}{lcc}\text { Figura 29- } & \text { Leitura de cor e } \\ \text { translucidez } & \text { sobre } & \text { fundo }\end{array}$ branco.

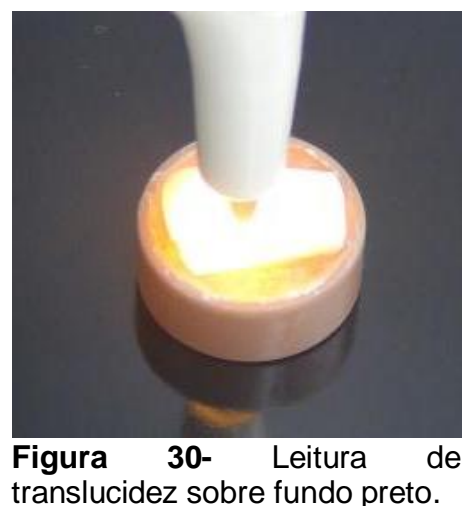

translucidez sobre fundo preto.

Após as leituras iniciais, todas as amostras (restauradas e grupos-controle) foram separadas aleatoriamente em 3 grupos $(n=10)$, conforme o tipo de envelhecimento ao qual foram submetidos. O primeiro grupo não foi submetido a nenhum tipo de envelhecimento e foi considerado Grupo controle, o segundo grupo foi mantido em água destilada, em estufa a $37^{\circ}$ C por um período de um ano e o terceiro grupo foi submetido à ciclagem termomecânica.

O desenho experimental do estudo ficou da seguinte forma: 


\begin{tabular}{|c|c|c|c|}
\hline Cerâmica & Espessura & Cimento & Tratamento \\
\hline \multirow{12}{*}{ IPS e-maxPress } & \multirow{6}{*}{$0,5 \mathrm{~mm}$} & \multirow{3}{*}{ Variolink II } & Controle \\
\hline & & & Água destilada \\
\hline & & & Ciclagem \\
\hline & & \multirow{3}{*}{ Rely-X U200 } & Controle \\
\hline & & & Água destilada \\
\hline & & & Ciclagem \\
\hline & \multirow{6}{*}{$1,0 \mathrm{~mm}$} & \multirow{3}{*}{ Variolink II } & Controle \\
\hline & & & Água destilada \\
\hline & & & Ciclagem \\
\hline & & \multirow{3}{*}{ Rely-X U200 } & Controle \\
\hline & & & Água destilada \\
\hline & & & Ciclagem \\
\hline \multirow{12}{*}{ IPS e-maxZirPress } & \multirow{6}{*}{$0,5 \mathrm{~mm}$} & \multirow{3}{*}{ Variolink II } & Controle \\
\hline & & & Água destilada \\
\hline & & & Ciclagem \\
\hline & & \multirow{3}{*}{ Rely-X U200 } & Controle \\
\hline & & & Água destilada \\
\hline & & & Ciclagem \\
\hline & \multirow{6}{*}{$1,0 \mathrm{~mm}$} & \multirow{3}{*}{ Variolink II } & Controle \\
\hline & & & Água destilada \\
\hline & & & Ciclagem \\
\hline & & \multirow{3}{*}{ Rely-X U200 } & Controle \\
\hline & & & Água destilada \\
\hline & & & Ciclagem \\
\hline
\end{tabular}

\subsection{Envelhecimento em água destilada}

Para o grupo mantido em água destilada por 1 ano, as amostras foram acondicionadas em recipientes individuais totalmente imersas (Figura 31) em água destilada e mantidas em estufa (Figura 32 ) a $37^{\circ} \mathrm{C}$ por um ano. Foram realizadas trocas de água semanais.

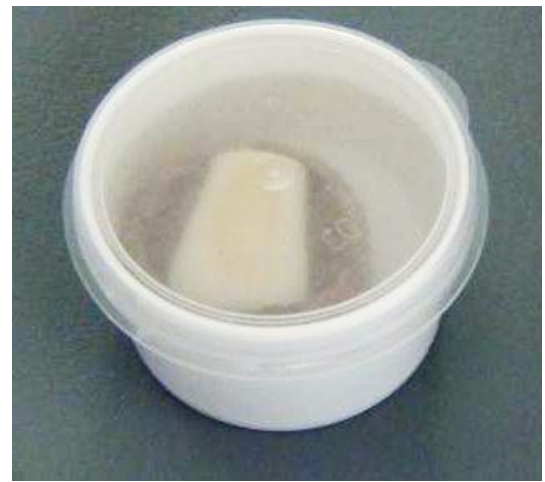

Figura 31- Corpo de prova acondicionado para envelhecimento em água destilada.

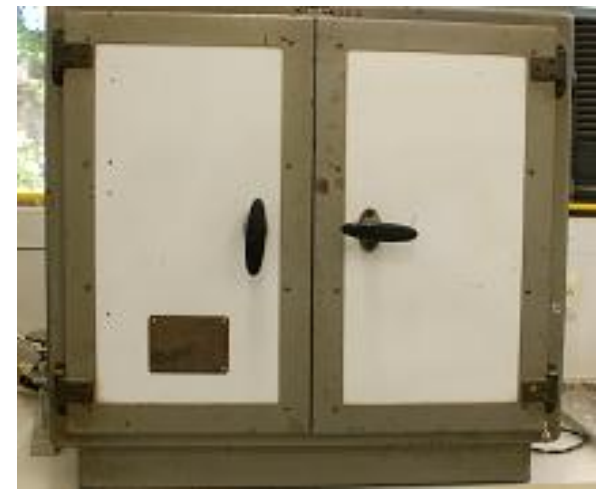

Figura 32- Estufa. 


\subsection{Ciclagem termomecânica}

A ciclagem termomecânica (ER 37000, Erios Equipamentos Técnicos e Científicos Ltda, São Paulo, SP, Brasil, Figura 33 e 34), foi realizada com força de 133N (De Boever J et al,1978; Fontijn-Tekamp FA et al, 2000) que corresponde à força de mordida de dentes anteriores em pacientes com oclusão normal. Foram realizados 1.200 .000 ciclos na frequência de $1.3 \mathrm{~Hz}$ (Komine et al, 2004) com temperatura variando de $5^{\circ} \mathrm{C}, 37^{\circ} \mathrm{C}$ e $55^{\circ} \mathrm{C}$. Cada faixa de temperatura ficou em contato com o espécime 35 segundos, sendo que cada ciclo completo do módulo térmico foi composto por 175 segundos.

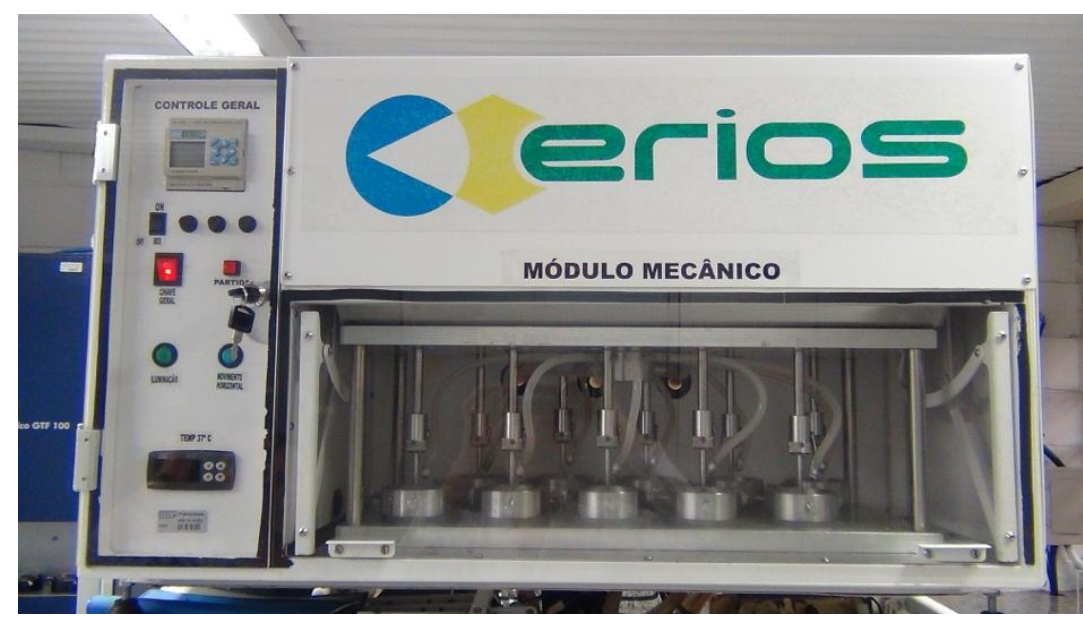

Figura 33- Equipamento de Fadiga termomecânica.

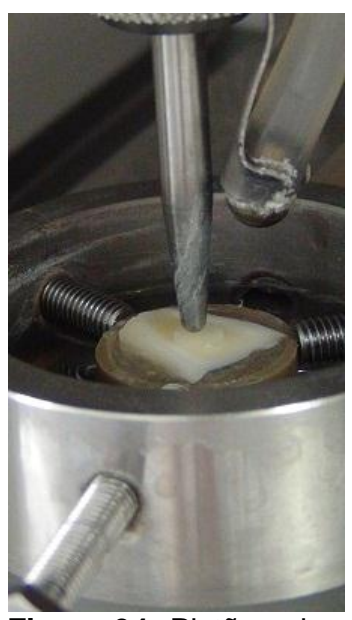

Figura 34- Pistão sobre laminado.

\subsection{Cálculo da Alteração de Cor}

Após os tratamentos dos diferentes grupos, novas leituras de cor e translucidez foram realizadas.

$\mathrm{O} \Delta \mathrm{E}$ foi calculado partir da seguinte fórmula (Pires-de-Souza et al., 2009):

$$
\Delta \mathrm{E}^{*}=\sqrt{\left(\Delta \mathrm{L}^{*}\right)^{2}+\left(\Delta \mathrm{a}^{*}\right)^{2}+\left(\Delta \mathrm{b}^{*}\right)^{2}}
$$

onde:

$\Delta \mathrm{E}^{*}=$ alteração de cor

$\Delta \mathrm{L}^{*}=$ diferença na luminosidade $\left(\mathrm{L}^{*}\right)$

$\Delta \mathrm{a}^{*}=$ diferença no eixo $\mathrm{a}^{*}$

$\Delta \mathrm{b}^{*}=$ diferença no eixo $\mathrm{b}^{*}$ 
A direção da diferença de cor é descrita pelas magnitudes e sinais algébricos dos componentes $\Delta \mathrm{L}^{*}, \Delta \mathrm{a}^{*}$ e $\Delta \mathrm{b}^{*}$ :

$$
\begin{aligned}
& \Delta \mathrm{L}^{*}=\mathrm{L}^{*} \mathrm{~F}-\mathrm{L}^{*}{ }_{\mathrm{I}} \\
& \Delta \mathrm{a}^{*}=\mathrm{a}^{*}{ }_{\mathrm{F}}-\mathrm{a}^{*}{ }_{\mathrm{I}} \\
& \Delta \mathrm{b}^{*}=\mathrm{b}^{*}{ }_{\mathrm{F}}-\mathrm{b}^{*}{ }_{\mathrm{I}}
\end{aligned}
$$

onde $L^{*}{ }_{1}, a^{*}{ }_{1}$ e $b^{*}{ }_{1}$ são referidos como medição inicial da cor e $L^{*}{ }_{F}, a^{*}{ }_{F}$ e $b^{*}{ }_{F}$ como medição final da cor.

\subsection{Cálculo da Translucidez}

A translucidez foi calculada através do Parâmetro de Translucidez (TP) que é calculado pela diferença dos valores de coordenadas de cor obtidos para uma amostra contra fundo branco e fundo preto, utilizando a seguinte fórmula (Bagis \& Turgut, 2012):

$$
T P^{*}=\sqrt{\left(L^{*}{ }_{p}-L^{*}{ }_{b}\right)^{2}+\left(a^{*}{ }_{p}-a^{*}{ }_{b}\right)^{2}+\left(b^{*}{ }_{p}-b^{*}{ }_{b}\right)^{2}}
$$

Onde $L^{*}{ }_{p}$ representa valores de luminosidade com fundo preto e $L^{*}{ }_{b}$ valores de luminosidade com fundo branco. Alterações de TP $(\Delta T P)$ foram calculados pela diferença entre os valores de TP antes e depois dos ensaios submetidos. Dessa forma, a variação de translucidez foi calculada pela seguinte fórmula:

$$
\Delta T P^{*}=T P_{\text {final }}-T_{\text {inicial }}
$$

Os dados coletados de alteração de cor e translucidez foram submetidos à análise estatística utilizando Analise de Variância (3-way ANOVA) e teste de Tukey, com nível de significância de 5\%.

\subsection{Grau de conversão}

Previamente a cimentação dos laminados cerâmicos, foi determinada a Irradiância Espectral do fotopolimerizador (FLASHlite 1401, Discus Dental) por um sensor acoplado a 
uma fibra óptica e esta ao Espectroradiômetro (Spectroradiometer Model USB 2000, Ocean Optimus, EUA, Figura 35). A ponteira do fotopolimerizador (FLASHlite 1401, Discus Dental) era posicionada em contato com o sensor e a irradiância espectral do fotopolimerizador era registrado. Os dados foram obtidos e integrados (Figura 36) determinando assim a irradiância total do fotopolimerizador no valor de $1423 \mathrm{~mW} / \mathrm{cm}^{2}$.

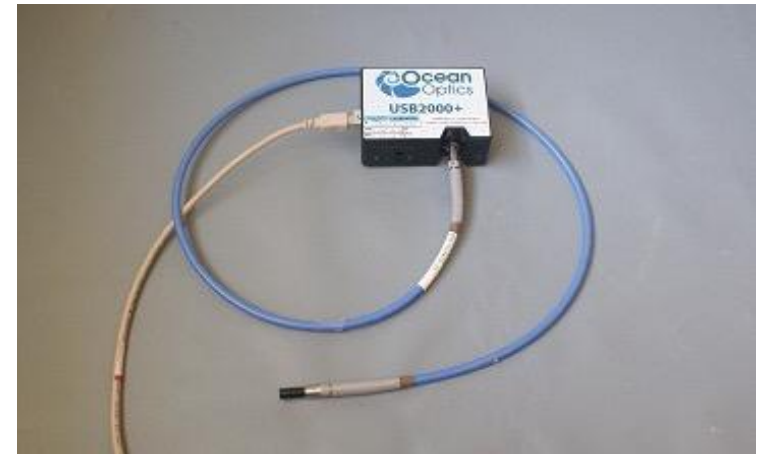

Figura 35- Fibra óptica e espectroradiômetro (Spectroradiometer Model USB 2000, Ocean Optimus, EUA).

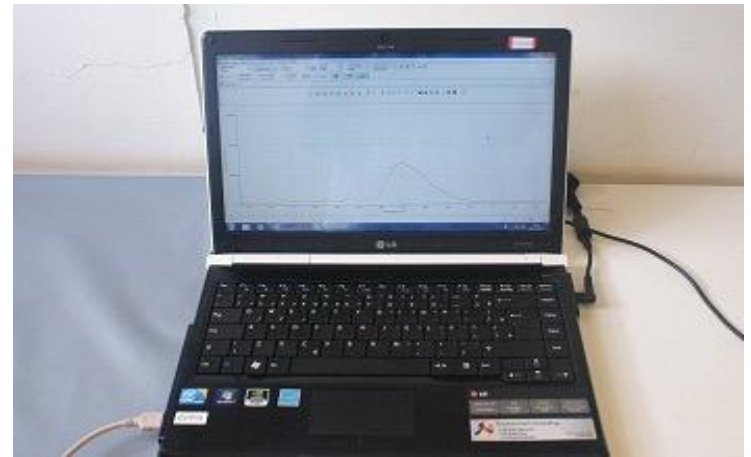

Figura 36- Dados coletados da irradiância espectral.

Para determinar o percentual de absorção da luz pelo laminado cerâmico, a potência transmitida foi medida por uma esfera integradora (Illumia, Labsphere, North Sutton, EUA). Para tal experimento a ponteira do fotopolimerizador (FLASHlite 1401, Discus Dental) foi acoplada na abertura da esfera integradora (Figura 37) e quando acionado, proporcionou a medida da potência espectral do fotopolimerizador $\left(I_{0}\right)$. Na sequência o laminado cerâmico foi posicionado na abertura da esfera (Figura 38). Com o fopolimerizador (FLASHlite 1401, Discus Dental) acionado sobre o laminado, uma nova medida potência espectral foi obtida $\left(I_{1}\right)$. A razão entre $I_{1}$ (quantidade de luz que efetivamente chega ao cimento) e $I_{0}$ (quantidade de luz que sai do fotopolimerizador) forneceu o percentual de transmissão da luz para cada sistema cerâmico e espessura do laminado. 


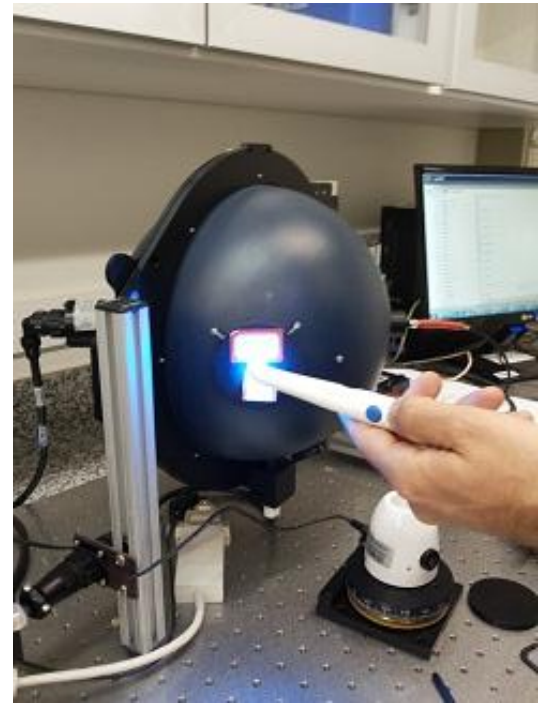

Figura 37- Medição do percentual de absorção de luz através da esfera integradora (Illumia, Labsphere, North Sutton, EUA).

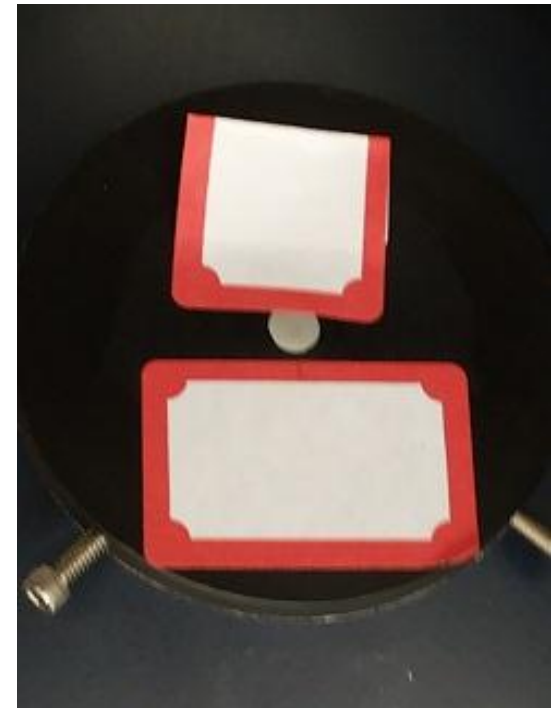

Figura 38- Posicionamento do corpo-de-prova de cerâmica na esfera integradora.

O grau de conversão dos cimentos resinosos foi avaliado com espectrômetro de infravermelho transformado por Fourier - FTIR (Nicolet 380, ThermoNicolet, EUA, Figura 41) empregando a técnica de transmissão (Figura 42). Para isso, foram confeccionados corpos de prova $(n=3)$ de dois tipos de cimento resinoso: cimento fotoativado (pasta base do Variolink II) e cimento autoadesivo (Rely-X U200). Para a realização do experimento foi utilizado filme plástico entre dente/cimento/cerâmica durante o procedimento de cimentação para que o cimento não aderisse à cerâmica e ao dente, e assim pudesse ser removido e analisado no FTIR. O cimento foi fotopolimerizado por 40 segundos com a ponteira do fotopolimerizador (FLASHlite 1401) em contato com a cerâmica. Em seguida, os corpos de prova de cimento foram removidos do contato da cerâmica e do dente e armazenados por 24h em caixas à prova de luz, a fim de evitar uma nova exposição à luz.

As amostras de cimentos polimerizadas foram acopladas a um dispositivo de acrílico composto por duas partes autorrosqueantes (Figura 39) que mantinha o corpo-de-prova do cimento em única posição (Figura 40) dentro do equipamento de FTIR. O feixe de luz passava pelo corpo-de-prova e realizava a análise por transmissão (Figura 42) com resolução de $1 \mathrm{~cm}^{-1}$. Este aparelho registra a banda de absorção das ligações aromáticas 
$(\operatorname{Abs}(\mathrm{C}-\mathrm{C}))$ e a banda de absorção das ligações duplas alifáticas $(\mathrm{Abs}(\mathrm{C}=\mathrm{C}))$. Os registros foram realizados em triplicata para cada corpo-de-prova.

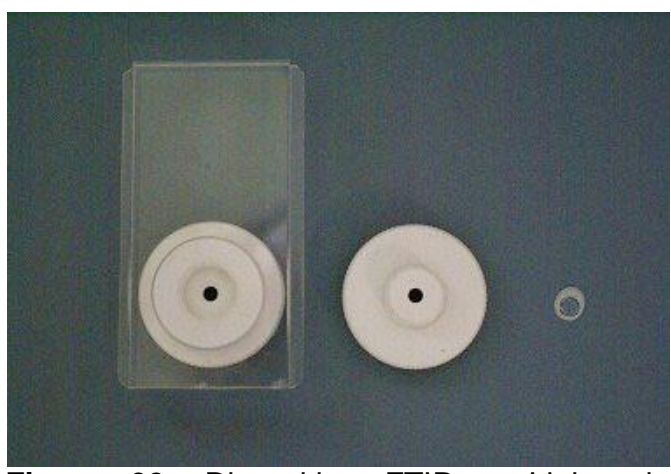

Figura 39- Dispositivo FTIR e Linha de Cimentação.

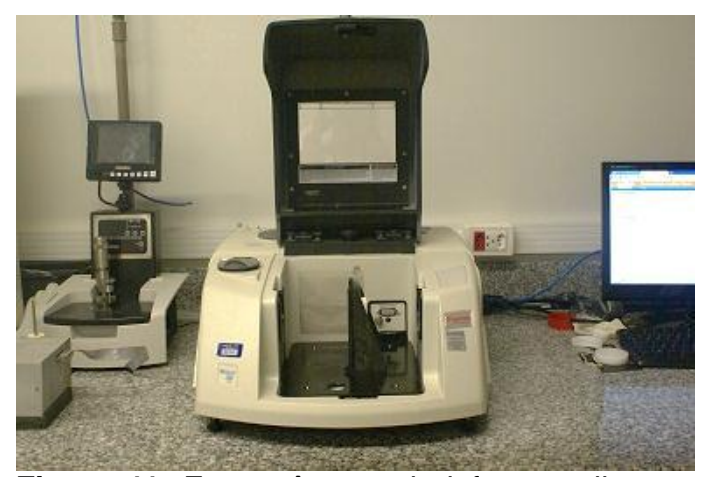

Figura 41- Espectrômetro de infravermelho por transformado por Fourier - FTIR (Nicolet 380, ThermoNicolet, EUA).

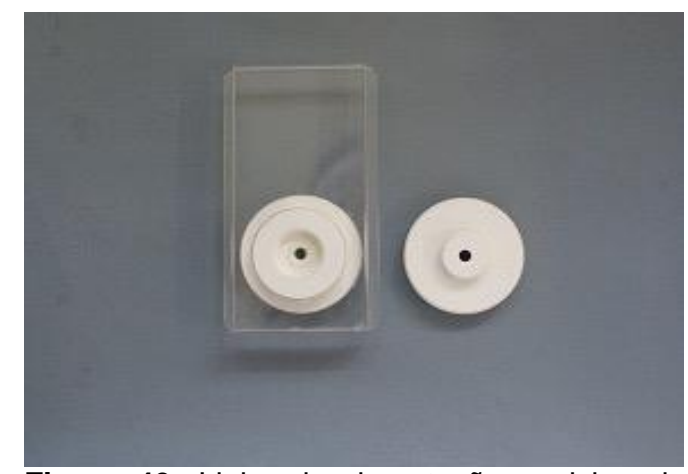

Figura 40- Linha de cimentação posicionada no dispositivo.

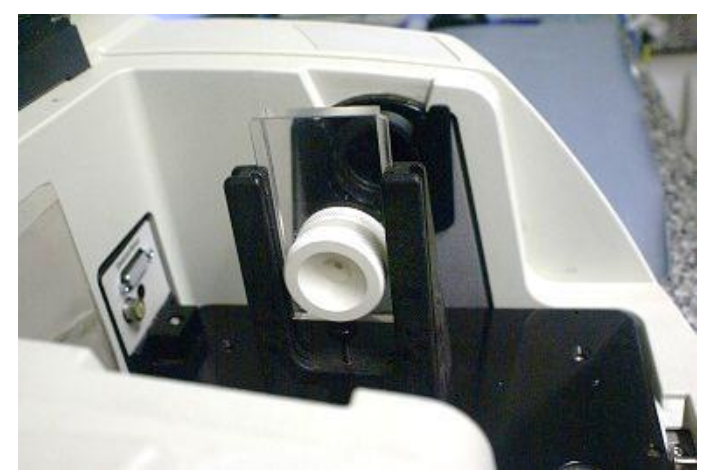

Figura 42- Técnica da transmissão.

A amostra do cimento não polimerizado foi obtido pela prensagem do cimento a partir de duas janelas de $\mathrm{CaF}_{2}$ e os mesmos procedimentos da análise do cimento polimerizado foram adotados para esta amostra.

Assim, foi calculada a percentagem de ligações duplas remanescentes (DLR) utilizando seguinte fórmula (Passos et al., 2013):

$\% \mathrm{DLR}=($ Abs $\mathrm{C}=\mathrm{C} / \mathrm{Abs} \mathrm{C}-\mathrm{C}$ para resina polimerizada $) \times 100$

(Abs $\mathrm{C}=\mathrm{C} /$ Abs $\mathrm{C}-\mathrm{C}$ para resina não polimerizada)

A percentagem do grau de conversão de ligações duplas (GC) a partir de FTIR foi calculada pela seguinte fórmula: 
Média e desvio padrão foram calculados a partir da triplicata de cada condição experimental e os dados foram submetidos à análise estatística (3-way ANOVA) e teste de Tukey com nível de significância de 95\%.

\subsection{Linha de Cimentação}

A análise da linha de cimentação foi realizada em 3 amostras de cada grupo, antes e depois do envelhecimento (controle e fadiga termomecânica). As amostras foram selecionadas aleatoriamente e seccionadas (Figura 43) ao meio com auxílio de um disco diamantado flexível montado em cortadeira metalográfica de precisão (Isomet 1000, Buehler, Figura 44). Após o seccionamento foram levadas ao estereomicroscópio de dupla coordenada (Nikon Measurescope, NipponKogaru, KK, Japão) para medição da linha de cimentação (Figura 45). Média e desvio padrão foram calculados a partir da triplicata de cada condição experimental e os dados foram submetidos à análise estatística utilizando Analise de Variância (3-way ANOVA) e teste de Tukey, com nível de significância de 95\%.

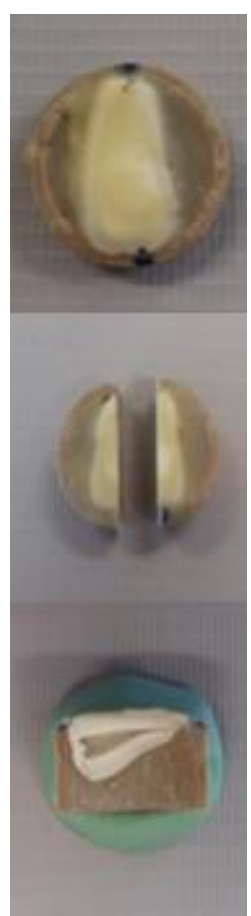

Figura 43- Sequência de preparo da amostra

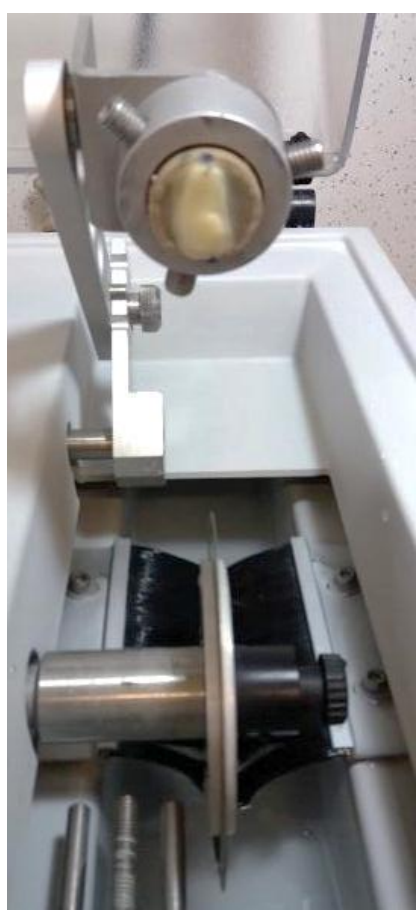

Figura 44- Secção da amostra

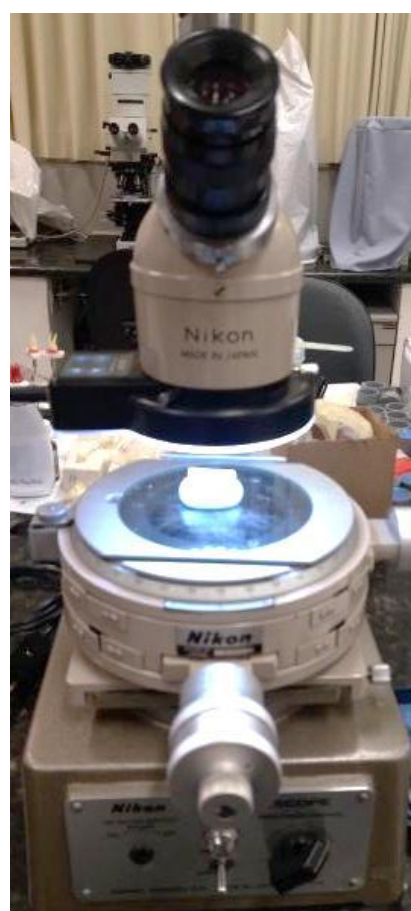

Figura 45- Posicionamento da amostra no estereomicroscópio 


\subsection{Grupos Controles}

Foram obtidos dois grupos-controle: um relacionado aos cimentos e outro aos dentes. Trinta dentes $(n=10)$ dos 270 dentes selecionados foram desgastados em dentina foram utilizados como grupo-controle, sobre os quais não foi fixado nenhum corpo de prova ou cimento. Vinte corpos de prova foram confeccionados com os dois cimentos resinosos usados no estudo ( $n=10)$ utilizando a mesma matriz descrita anteriormente, em uma só espessura $(0,5 \mathrm{~mm})$. Para isso, as pastas base e catalisadora do cimento autoadesivo foram dispensadas em comprimentos iguais e manipuladas da mesma maneira quando o cimento foi utilizado para fixação das peças. O cimento fotopolimerizável foi dispensado diretamente sobre a matriz. Em seguida os cimentos foram fotopolimerizados (FLASHlite 1401, Discus Dental) por 40 segundos sobre uma lâmina de vidro apoiados a matriz.

Tanto os dentes foram mantidos em a $37^{\circ} \mathrm{C}$ por $24 \mathrm{~h}$ e as amostras de cimentos foram mantidas em recipientes a prova de luz por $24 \mathrm{~h}$. Dentes e amostras de cimentos foram submetidos ao diferentes protocolos de envelhecimento descrito anteriormente. 
4. Resultados 



\section{REsultados}

\subsection{Grau de Conversão}

A Tabela 2 mostra a comparação das médias (3-way ANOVA, Tukey, $p<0,05)$, em porcentagem, do grau de conversão dos diferentes cimentos resinosos através de laminados em diferentes espessuras.

Tabela 2- Comparação das médias (3-way ANOVA, Tukey, $p<0,05$ ) do grau de conversão dos cimentos resinosos dos para grupos estudados.

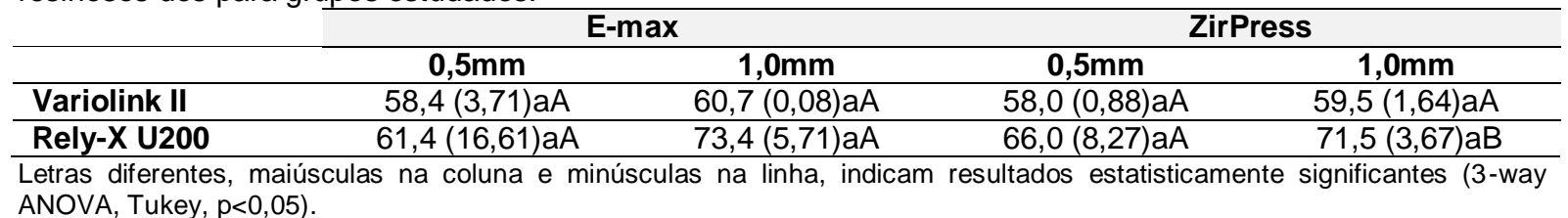

Observa-se que o cimento autoadesivo apresentou maior grau de conversão que o fotoativado $(p<0,05)$ quando utilizada a cerâmica Zirpress. Verifica-se que o tipo de sistema cerâmico é um fator significante para o grau de conversão do cimento, mas a espessura do laminado cerâmico não.

O gráfico 1 mostra percentual de transmissão da luz para cada sistema cerâmico e espessura do laminado.

Gráfico 1- Imagem ilustrativa do percentual de luz através dos materiais e espessuras testados.

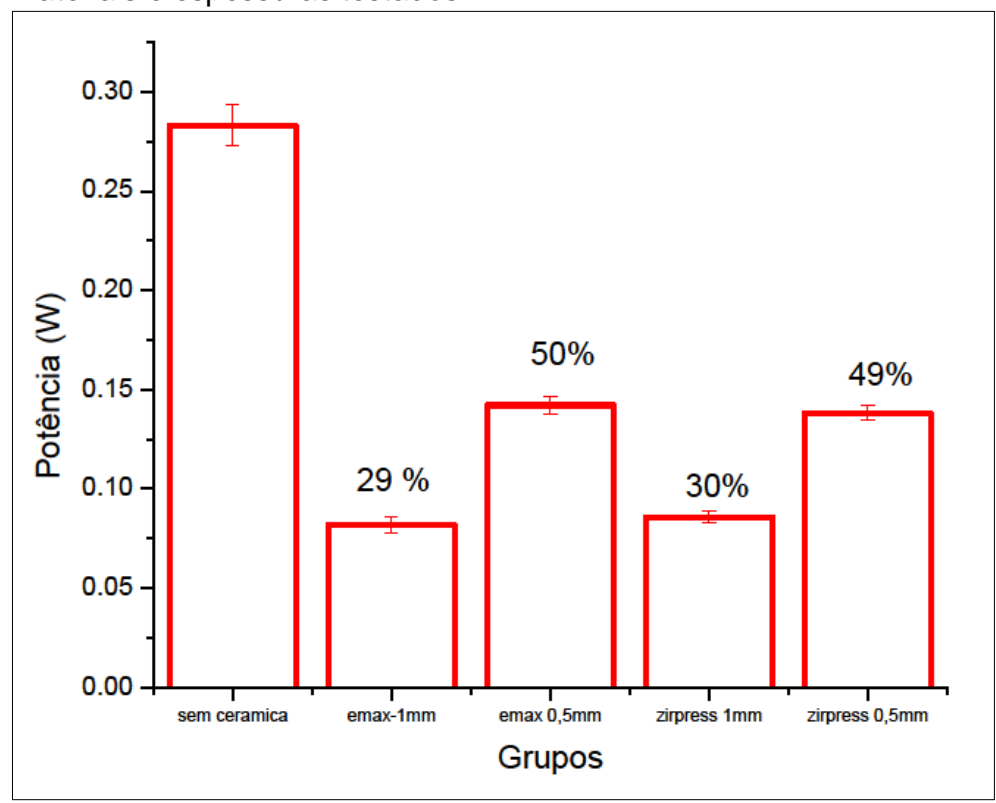


De acordo com os resultados, quanto maior a espessura do laminado, menor a transmissão de luz ao cimento resinoso. Não houve diferença estatisticamente significante ( $p>0,05)$ em relação aos sistemas cerâmicos estudados.

\subsection{Alteração de Cor}

Os resultados para alteração de cor podem ser vistos na Tabela 3 e Gráfico 2. A Tabela 3 mostra a comparação das médias (3-way ANOVA, Tukey, $p<0,05)$ da alteração de cor dos grupos, para cada tipo de cimento utilizado, em relação aos sistemas cerâmicos, espessuras dos laminados e o tipo de envelhecimento.

Tabela 3- Comparação das médias e desvio padrão (3-way ANOVA, teste de Tukey, $p<0,05)$ da alteração de cor dos grupos estudados.

\begin{tabular}{|c|c|c|c|c|c|}
\hline & & & Ciclagem & Envelhecimento & Controle \\
\hline \multirow{6}{*}{ Variolink II } & \multirow{2}{*}{ E-max } & $0,5 \mathrm{~mm}$ & $3,75(2,12) \mathrm{aA}$ & $2,74(0,87) \mathrm{aA}$ & $3,14(2,94)$ aABD \\
\hline & & $1,0 \mathrm{~mm}$ & $1,56(0,83)$ aA & $2,39(1,83) \mathrm{aA}$ & $1,03(0,30)$ aAC \\
\hline & \multirow{2}{*}{ Zirpress } & $0,5 \mathrm{~mm}$ & $1,34(0,55) \mathrm{aA}$ & $2,61(0,97) a A$ & $5,14(0,40) b B D$ \\
\hline & & $1,0 \mathrm{~mm}$ & $1,99(1,40)$ aA & $3,24(1,64)$ aA & $1,58(0,86)$ aACE \\
\hline & \multicolumn{2}{|c|}{ Cimento } & * & $3,47(0,93)$ bA & $0,88(0,34) \mathrm{aC}$ \\
\hline & \multicolumn{2}{|c|}{ Dentes } & $5,20(4,24) a B$ & $9,23(4,15) \mathrm{bB}$ & $4,07(1,89)$ aDE \\
\hline \multirow{6}{*}{$\begin{array}{c}\text { Rely-X } \\
\text { U200 }\end{array}$} & \multirow{2}{*}{ E-max } & $0,5 \mathrm{~mm}$ & $2,92(2,00)$ aAB & $4,02(1,36)$ aA & $2,74(1,61) \mathrm{aAB}$ \\
\hline & & $1,0 \mathrm{~mm}$ & $1,26(1,14)$ aA & $1,16(0,76) \mathrm{aB}$ & $0,90(0,93) \mathrm{aA}$ \\
\hline & \multirow{2}{*}{ Zirpress } & $0,5 \mathrm{~mm}$ & $1,78(1,32) a A$ & $6,46(1,71) b A$ & $1,15(0,39) a A$ \\
\hline & & $1,0 \mathrm{~mm}$ & $1,51(0,89) \mathrm{aA}$ & $3,81(1,39) b A$ & $2,97(1,61)$ abAB \\
\hline & \multicolumn{2}{|c|}{ Cimento } & * & $30,56(2,34) \mathrm{bC}$ & $1,15(0,37) \mathrm{aA}$ \\
\hline & \multicolumn{2}{|c|}{ Dentes } & $5,20(4,24) \mathrm{aB}$ & $9,23(4,15) b D$ & $4,07(1,89) \mathrm{aB}$ \\
\hline
\end{tabular}

Letras diferentes, minúsculas na linha e maiúsculas na coluna, para cada cimento testado e dentes, indicam diferença estatisticamente significante $(p<0,05)$.

${ }^{*}$ As amostras fraturaram e os resultados não foram analisados

Não houve diferença estatisticamente significante $(p<0,05)$ quando as amostras foram cimentadas com cimento Variolink II após os tratamentos testados, exceto para o grupo Zirpress $0,5 \mathrm{~mm}$, que apresentou a maior alteração de cor no grupo Controle com diferença estatisticamente significante $(p<0,05)$ em relação aos demais tratamentos, que não apresentaram diferença entre si $(p>0,05)$.

Os dentes apresentaram maior alteração de cor que os materiais testados. A alteração foi maior após envelhecimento em água destilada, resultados diferentes $(p<0,05)$ estatisticamente em relação aos grupos Controle e Ciclagem, sem diferença $(p>0,05)$ entre si. 
As amostras de cimento não resistiram à ciclagem termomecânica e fraturaram durante o ensaio (figuras 46 e 47), o que inviabilizou a comparação dos resultados dessa variável. Amostras de cimento submetidas a envelhecimento em água destilada apresentaram maior alteração de cor $(p<0,05)$ que o grupo Controle.

Quanto ao cimento Rely-X U200, grupos obtidos com cerâmica E-max Press, nas duas espessuras, não apresentaram diferença estatisticamente significante $(p>0,05)$ em função do envelhecimento a que foram submetidos. A cerâmica Zirpress, em ambas as espessuras, apresentou maior alteração de cor para os grupos submetidos ao envelhecimento com água destilada, com diferença estatisticamente significante $(p<0,05)$ em relação aos grupos submetidos à Ciclagem e Controle, que não apresentaram diferença $(p>0,05)$ entre si.

Amostras de cimento apresentaram grande alteração de cor após Envelhecimento em água destilada, resultado diferente estatisticamente $(p<0,05)$ do Controle.

Quanto à comparação dos tratamentos a que as amostras foram submetidas, nas diferentes espessuras dos sistemas cerâmicos, observou-se que espécimes de Variolink II não apresentaram diferença $(p>0,05)$ entre as espessuras nos grupos tratados. Essas amostras foram diferentes $(p<0,05)$ apenas dos dentes, que apresentaram a maior alteração de cor.

Quanto ao grupo Controle, dentes restaurados com E-max Press de $1 \mathrm{~mm}$ apresentaram diferença estatisticamente significante $(p<0,05)$ em relação ao grupo restaurado com Zirpress de $0,5 \mathrm{~mm}$, que apresentou resultado diferente estatisticamente $(p<0,05)$ de Zirpress de $1,0 \mathrm{~mm}$, mas semelhante $(p>0,05)$ a E-max Press de $1,0 \mathrm{~mm} . A$ menor alteração de cor nesse grupo ocorreu para amostras de cimento, resultado diferente $(p<0,05)$ de E-max Press e Zirpress de 0,5mm e em relação aos Dentes, que apresentaram a maior alteração de cor, resultados diferentes $(p<0,05)$ em relação aos grupos $E$-max Press e Zirpress de $1,0 \mathrm{~mm}$.

Quanto ao cimento Rely-X U200, não houve diferença estatisticamente significante $(p>0,05)$ quando as amostras restauradas com cerâmicas foram tratadas com ciclagem 
termomecânica e no grupo Controle. As diferenças $(p<0,05)$ nesses grupos ocorreram em relação aos dentes, que apresentaram maior alteração de cor, com exceção ao grupo Zirpress $1,0 \mathrm{~mm}$ no grupo Controle, que apresentou resultado semelhante $(p>0,05)$ a todos os grupos testados.

Quando as amostras foram submetidas a envelhecimento em água destilada, verificou-se que as restaurações de E-max Press de 1,0mm apresentaram menor alteração de cor, resultados diferentes estatisticamente $(p<0,05)$ de todos os outros grupos. Os grupos restaurados com lâminas não apresentaram diferença $(p>0,05)$ entre si, mas esses resultados foram diferentes $(p<0,05)$.

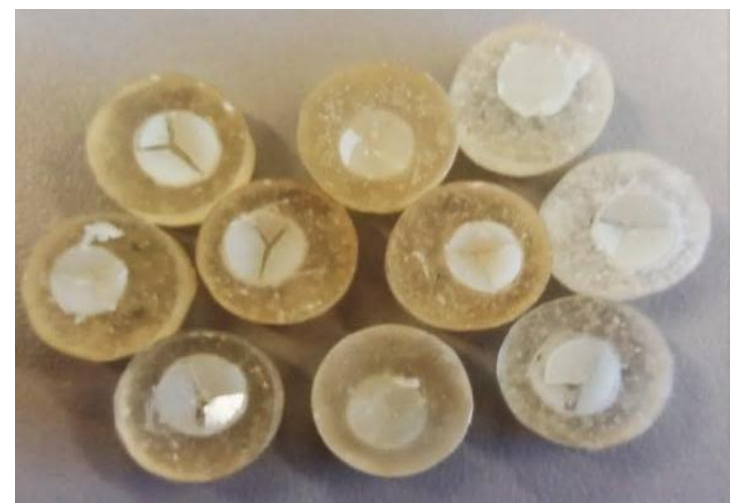

Figura 46- Corpos de prova fraturados.

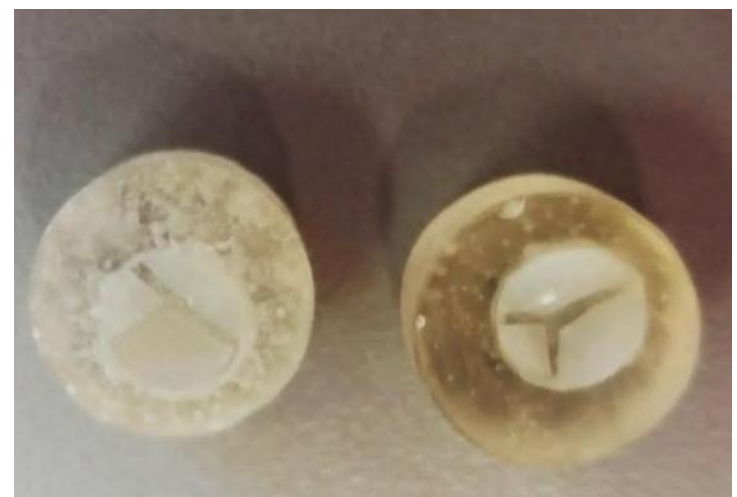

Figura 47- Imagem ampliada dos corpos de prova fraturados.

No gráfico 2 é possível visualizar a alteração de cor dos materiais testados em função dos ensaios realizados e é possível comparar o desempenho dos cimentos, para cada sistema cerâmico utilizado. 
Gráfico 2- Alteração de cor dos grupos estudados.

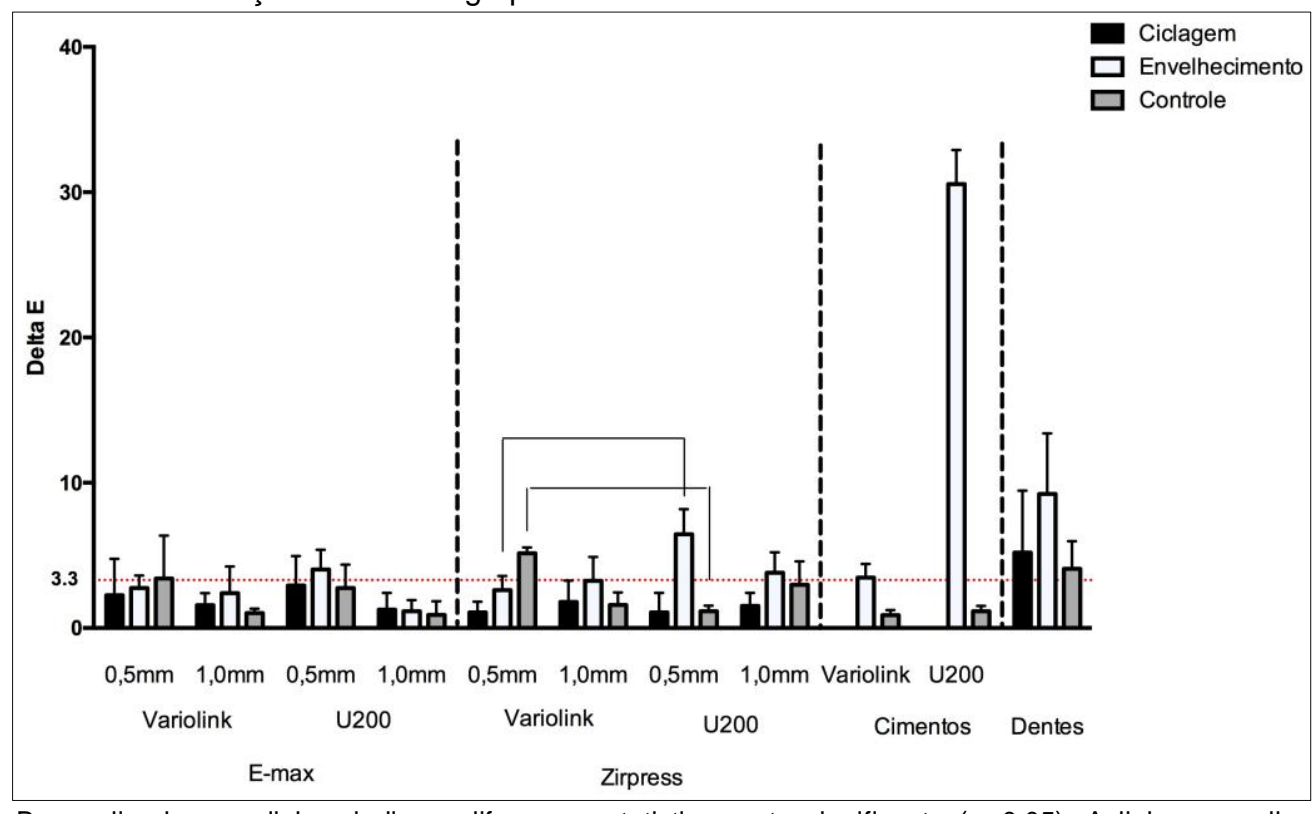

Barras ligadas por linhas indicam diferença estatisticamente significante $(p<0,05)$. A linha vermelha representa o valor crítico de alteração de cor clinicamente significante (Garcia et al, 2013; Silami et al, 2016).

Verificou-se que não houve diferença estatisticamente significante $(p>0,05)$ entre os cimentos fotoativado (Variolink II) e dual (Rely-X U200), exceto para os grupos da cerâmica Zirpress de 0,5mm, que apresentou maior alteração de cor, estatisticamente diferente $(p<0,05)$ quando cimentadas com cimento Rely-X U200 após envelhecimento em água destilada em comparação com cimento Variolink II. Este apresentou maior alteração de cor $(p<0,05)$ que Rely-X U200 no grupo Controle, quando utilizados para cimentação de peças de Zirpress 0,5mm.

\subsection{Alterações nas Coordenadas de Cor}

\section{- Delta L}

Os resultados para alteração de cor podem ser vistos na Tabela 4 e Gráfico 3.

Na Tabela 4 é possível avaliar a comparação das médias e desvios padrão (3-way ANOVA, Tukey, $p<0,05)$ da alteração de Delta $L$ dos grupos, para cada tipo de cimento utilizado, em relação aos sistemas cerâmicos, espessuras dos laminados e o tipo de envelhecimento. 
Tabela 4- Comparação das médias e desvio padrão (3-way ANOVA, teste de Tukey, $p<0,05)$ da alteração de Delta $L$ dos grupos estudados.

\begin{tabular}{|c|c|c|c|c|c|}
\hline & & & Ciclagem & Envelhecimento & Controle \\
\hline \multirow{6}{*}{ Variolink II } & \multirow{2}{*}{ E-max } & $0,5 \mathrm{~mm}$ & $-3,34(1,88) a A B$ & $-2,29(1,20) a A$ & $2,61(3,03) \mathrm{bAB}$ \\
\hline & & $1,0 \mathrm{~mm}$ & $-1,09(1,14) a b A$ & $-7,16(1,62) a A$ & $0,65(0,33) \mathrm{bBCD}$ \\
\hline & \multirow{2}{*}{ Zirpress } & $0,5 \mathrm{~mm}$ & $0,15(1,15) \mathrm{aA}$ & $-2,41(1,02) b A$ & $-4,85(0,22) \mathrm{cB}$ \\
\hline & & $1,0 \mathrm{~mm}$ & $-1,41(1,75) \mathrm{aA}$ & $-2,59(1,83) a A$ & $-1,34(0,76)$ aCD \\
\hline & \multicolumn{2}{|c|}{ Cimento } & * & $-0,30(1,31) a A$ & $-0,43(0,57)$ aCD \\
\hline & \multicolumn{2}{|c|}{ Dentes } & $-4,36(4,60) a B$ & $-6,99(4,99) b B$ & $0,13(3,40) \mathrm{cAD}$ \\
\hline \multirow{6}{*}{$\begin{array}{c}\text { Rely-X } \\
\text { U200 }\end{array}$} & \multirow{2}{*}{ E-max } & $0,5 \mathrm{~mm}$ & $-0,14(3,03) a A$ & $-3,30(1,66) a A B$ & $-0,61(1,62) a A$ \\
\hline & & $1,0 \mathrm{~mm}$ & $-0,11(1,57) a A$ & $-0,81(0,95) \mathrm{aA}$ & $-0,11(1,14) a A$ \\
\hline & \multirow{2}{*}{ Zirpress } & $0,5 \mathrm{~mm}$ & $0,23(0,77)$ aA & $-5,85(2,10) a B C$ & $-1,05(0,44) a A$ \\
\hline & & $1,0 \mathrm{~mm}$ & $0,77(1,08) a A$ & $-2,97(2,29) \mathrm{bAB}$ & $-2,47(1,67) b A$ \\
\hline & \multicolumn{2}{|c|}{ Cimento } & * & $-6,52(1,01) \mathrm{aC}$ & $-0,15(0,80) a A$ \\
\hline & \multicolumn{2}{|c|}{ Dentes } & $-4,36(4,60) a B$ & $-6,99(4,99) b C$ & $0,13(3,40) \mathrm{cA}$ \\
\hline
\end{tabular}

Letras diferentes, minúsculas na linha e maíusculas na coluna, para cada cimento testado e dentes, indicam diferença estatisticamente significante $(p<0,05)$

${ }^{*}$ As amostras fraturaram e os resultados não foram analisados

Todas as amostras submetidas a envelhecimento em água destilada apresentaram Delta L negativo, independente do cimento testado, demonstrando que houve "escurecimento" após o tratamento. Esses resultados foram diferentes estatisticamente $(p<0,05)$, quando analisado o cimento Variolink II no grupo Controle, em relação aos demais tratamentos, quando comparadas as cerâmicas E-max Press com 0,5 e 1,0mm. Para a cerâmica Zirpress, houve diferença significante $(p<0,05)$ entre todos os tratamentos quando a espessura foi de $0,5 \mathrm{~mm}$.

Quanto ao cimento Rely-X U200, os sistemas de envelhecimento não foram significantes $(p>0,05)$ para E-max Press em nenhuma das espessuras testadas e Zirpress de $0,5 \mathrm{~mm}$. Para Zirpress de 1,0 mm houve menor alteração de Delta $L$ no grupo Ciclagem, diferente estatisticamente $(p<0,05)$ dos demais grupos, que não apresentaram diferença entre si $(p>0,05)$. Não houve diferença significante $(p>0,05)$ para o Cimento isoladamente e o tipo de envelhecimento foi significante $(p<0,05)$ para todos os grupos tratados de dentes.

O tipo de cerâmica e sua espessura não foram fatores significantes $(p>0,05)$ para alteração de Delta L das amostras cimentadas com Rely-X U200. Houve diferença significante $(p<0,05)$ apenas em relação ao Cimento (para todas as amostras do Grupo Envelhecimento, com exceção de Zirpress $0,5 \mathrm{~mm}$ ) e Dentes (todas as amostras submetidas à Ciclagem e a Envelhecimento em água destilada, com exceção de Zirpress 
de $0,5 \mathrm{~mm})$. Não houve diferença estatisticamente significante $(p>0,05)$ entre amostras cimentadas com Rely-X U200 do Grupo Controle.

O gráfico 3 mostra a alteração de Delta L (luminosidade) dos materiais e cimentos testados, além do grupo de dentes, em relação aos testes realizados para cada sistema cerâmico.

Gráfico 3- Alteração de Delta L dos grupos estudados.

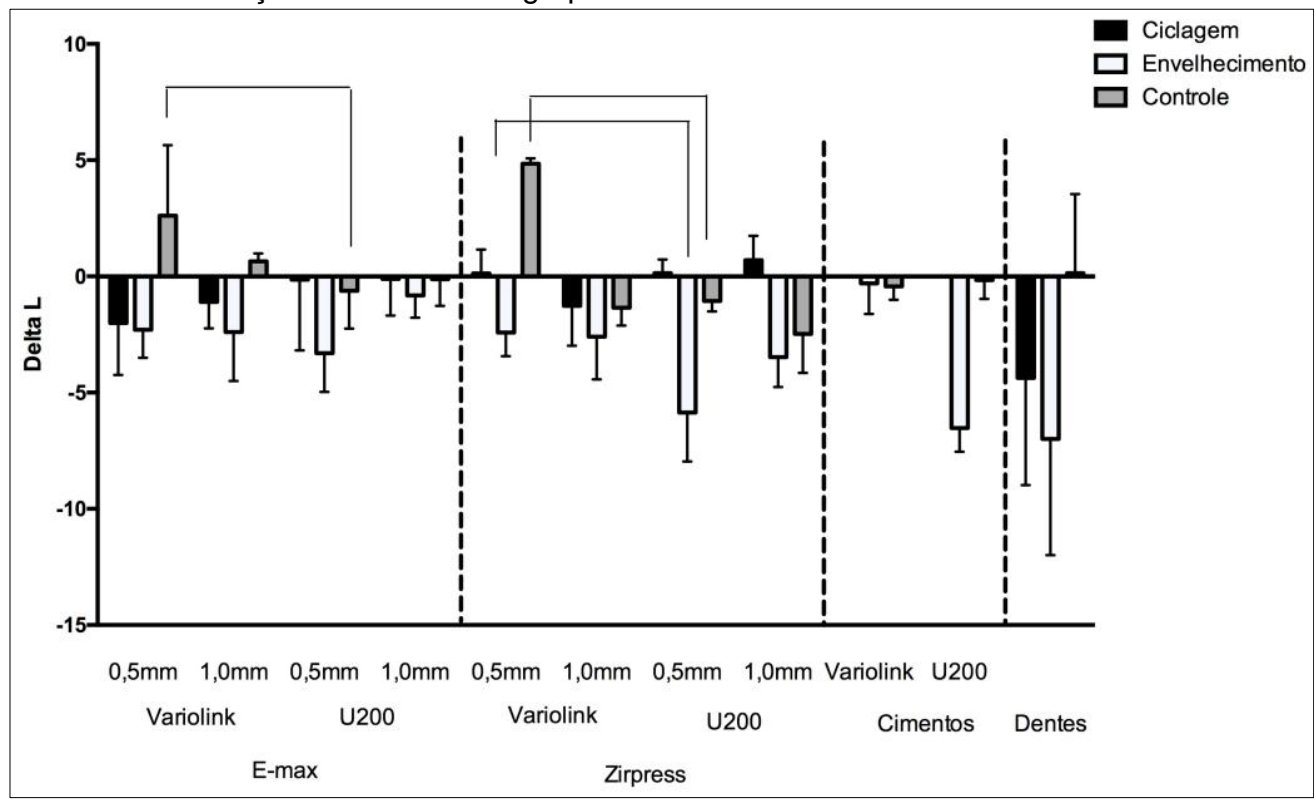

Barras ligadas por linhas indicam diferença estatisticamente significante $(p<0,05)$.

Na comparação de Delta L entre os cimentos fotoativado (Variolink II) e dual (RelyX U200), verificou-se que houve maior alteração da coordenada $L$ quando a restauração de E-max Press de 0,5mm foi cimentada com Variolink II, diferença estatisticamente significante $(p<0,05)$ em relação ao cimento Rely-X U200, no grupo Controle. Quanto à restauração com Zirpress, houve alteração de Delta $L$ significante $(p<0,05)$ na espessura de 0,5mm, quando cimentada com Rely-X U200 após Envelhecimento em água destilada e no grupo Controle, quando cimentada com Variolink II. Os demais grupos não apresentaram diferença estatisticamente significante $(p>0,05)$ entre si quando comparados em função do agente cimentante. 
Os resultados para alteração de cor podem ser vistos na Tabela 5 e Gráfico 4.

A Tabela 5 mostra a comparação das médias e desvios padrão (3-way ANOVA, Tukey, $p<0,05)$ da alteração de Delta a dos grupos, para cada tipo de cimento resinoso testado, variando o tipo de sistema cerâmico, a espessura dos laminados e o tipo de envelhecimento a que foram submetidos.

Tabela 5- Comparação das médias e desvio padrão (3-way ANOVA, teste de Tukey, p<0,05) da variação do Delta a dos grupos estudados.

\begin{tabular}{|c|c|c|c|c|c|}
\hline & & & \multirow{2}{*}{$\begin{array}{c}\text { Ciclagem } \\
-0,01(0,39) a A\end{array}$} & \multirow{2}{*}{$\begin{array}{c}\text { Envelhecimento } \\
0,80(0,27) \mathrm{bA}\end{array}$} & \multirow{2}{*}{$\begin{array}{c}\text { Controle } \\
-0,22(0,29) a A\end{array}$} \\
\hline \multirow{6}{*}{ Variolink II } & \multirow{2}{*}{ E-max } & $0,5 \mathrm{~mm}$ & & & \\
\hline & & $1,0 \mathrm{~mm}$ & $-0,32(0,17) a A$ & $0,38(0,44) \mathrm{bA}$ & $0,04(0,09) a b A$ \\
\hline & \multirow{2}{*}{ Zirpress } & $0,5 \mathrm{~mm}$ & $-0,27(0,34) a A$ & $0,35(0,21) \mathrm{aA}$ & $0,42(1,23) a A$ \\
\hline & & $1,0 \mathrm{~mm}$ & $-0,06(0,29) a A$ & $0,35(0,64)$ aA & $-0,27(0,37)$ aA \\
\hline & \multicolumn{2}{|c|}{ Cimento } & ${ }^{*}$ & $1,73(0,25) \mathrm{aB}$ & $0,33(0,22) \mathrm{bA}$ \\
\hline & \multicolumn{2}{|c|}{ Dentes } & $0,40(1,06) \mathrm{aA}$ & $1,99(1,42) b B$ & $-1,26(1,20) c B$ \\
\hline \multirow{6}{*}{ Rely-X U200 } & \multirow{2}{*}{ E-max } & $0,5 \mathrm{~mm}$ & $-0,36(0,35) a A$ & $0,16(0,24) a b A$ & $0,86(0,59) \mathrm{bA}$ \\
\hline & & $1,0 \mathrm{~mm}$ & $-0,03(0,22) a A$ & $0,24(0,13) a A$ & $-0,14(0,11) a B$ \\
\hline & \multirow{2}{*}{ Zirpress } & $0,5 \mathrm{~mm}$ & $0,04(0,33) a A$ & $0,22(0,26) \mathrm{aA}$ & $0,08(0,17) \mathrm{aAB}$ \\
\hline & & $1,0 \mathrm{~mm}$ & $-0,34(0,19) a A$ & $0,12(0,34) \mathrm{aA}$ & $0,01(0,30) \mathrm{aAB}$ \\
\hline & \multicolumn{2}{|c|}{ Cimento } & * & $2,46(1,83) \mathrm{aB}$ & $0,17(0,37) \mathrm{bAB}$ \\
\hline & \multicolumn{2}{|c|}{ Dentes } & $0,40(1,06) \mathrm{aA}$ & $1,99(1,42) b B$ & $-1,26(1,20) c C$ \\
\hline
\end{tabular}

Letras diferentes, minúsculas na linha e maíusculas na coluna, para cada cimento testado e dentes, indicam diferença estatisticamente significante $(p<0,05)$

${ }^{*}$ As amostras fraturaram e os resultados não foram analisados

Analisando-se o efeito do envelhecimento sobre o cimento Variolink II, observa-se que dentes restaurados com E-max Press, independente da espessura testada, quando submetidos a envelhecimento em água destilada, apresentaram maior alteração de Delta a, resultados diferentes estatisticamente $(p<0,05)$ das amostras submetidas à Ciclagem. Quando a espessura de $0,5 \mathrm{~mm}$ foi testada, o resultado foi diferente estatisticamente $(p<0,05)$ também do grupo Controle, o mesmo não ocorrendo $(p>0,05)$ quando a espessura era de 1,0mm, que também não apresentou diferença em relação ao grupo Ciclagem para nenhuma espessura. Não houve diferença significante $(p>0,05)$ para os tipos de envelhecimento para o grupo Zirpress, em nenhuma espessura.

Amostras de cimento Variolink II apresentaram maior alteração de Delta a $(p<0,05)$ quando submetidos a Envelhecimento em água destilada do que no grupo Controle e os dentes apresentaram diferença estatisticamente significante $(p<0,05)$ para todos os tipos de envelhecimento. 
Quanto ao cimento Rely-X U200, houve diferença estatisticamente significante $(p<0,05)$ apenas quando o laminado cerâmico foi obtido com E-max Press na espessura de $0,5 \mathrm{~mm}$, com maior alteração para o Grupo Controle, diferente estatisticamente $(p<0,05)$ do grupo Ciclagem e sem diferença $(p>0,05)$ do grupo Envelhecimento, que não apresentaram diferença $(p>0,05)$ entre si. Todos os demais grupos restaurados não apresentaram diferença $(p>0,05)$ entre os tipos de envelhecimento. O cimento e os dentes apresentaram diferença estatisticamente significante $(p<0,05)$ entre os três tipos de envelhecimento.

Comparando-se os efeitos dos tipos de envelhecimento sobre os diferentes sistemas cerâmicos e espessuras, para cada tipo de cimento, verificou-se que a Ciclagem não foi fator significante para alteração de Delta a nos grupos testados $(p>0,05)$. Quando submetido ao Envelhecimento em água destilada, não houve diferença entre os sistemas cerâmicos em nenhuma espessura $(p>0,05)$; houve apenas diferença significante $(p<0,05)$ em relação aos respectivos cimentos e aos dentes. Quanto ao Grupo Controle, quando utilizado o cimento Variolink II, observou-se que houve diferença significante $(p<0,05)$ dos dentes em relação a todos os outros grupos testados.

Quanto ao cimento Rely-X U200, houve diferença significante $(p<0,05)$ entre os grupos restaurados com E-max Press de 0,5 e 1,0mm. Não foram observadas outras diferenças $(p>0,05)$ em relação aos grupos restaurados e ao cimento. Todos os grupos, porém, apresentaram diferença estatisticamente significante $(p<0,05)$ em relação aos dentes.

O gráfico 4 mostra a alteração de Delta a (cromaticidade no eixo verde-vermelho) dos materiais e cimentos testados, além do grupo de dentes, em relação aos testes realizados para cada sistema cerâmico. 
Gráfico 4- Alteração de Delta a dos grupos estudados.

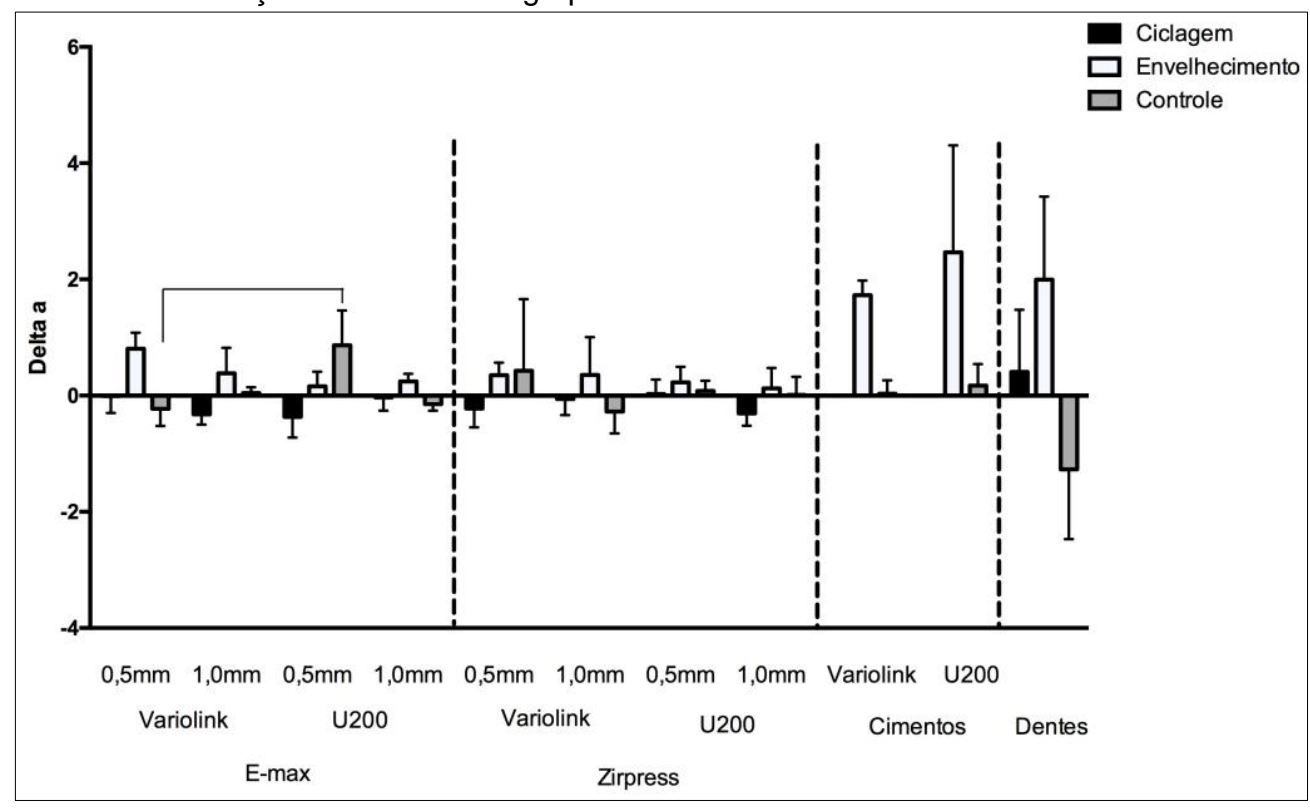

Barras ligadas por linhas indicam diferença estatisticamente significante $(p<0,05)$.

Na comparação de Delta a entre os cimentos resinosos, verificou-se que houve diferença estatisticamente significante $(p<0,05)$ para as amostras de $E$-max Press de 0,5mm do Grupo Controle, sendo que a maior alteração ocorreu após cimentação com cimento Rely-X U200. Para todas as demais comparações não houve diferença estatisticamente significante $(p>0,05)$ em função do cimento resinoso.

\section{- Delta b}

Os resultados para alteração de cor podem ser vistos na Tabela 6 e Gráfico 5.

A Tabela 6 mostra a comparação das médias e desvios padrão (3-way ANOVA, Tukey, $p<0,05)$ da alteração de Delta b para cada tipo de cimento testado, comparando os sistemas cerâmicos, as espessuras dos laminados e o tipo de envelhecimento a que foram submetidos. 
Tabela 6- Comparação das médias e desvio padrão (3-way ANOVA, teste de Tukey, $p<0,05$ ) da variação do Delta b dos grupos estudados.

\begin{tabular}{|c|c|c|c|c|c|}
\hline & & & Ciclagem & Envelhecimento & Controle \\
\hline \multirow{6}{*}{ Variolink II } & & $0,5 \mathrm{~mm}$ & $-1,43(1,35)$ aA & $0,75(0,65) \mathrm{bA}$ & $0,27(064) a b A$ \\
\hline & E-max & $1,0 \mathrm{~mm}$ & $-0,13(0,78) a A B$ & $-0,50(1,20) a A$ & $0,75(0,23)$ aA \\
\hline & & $0,5 \mathrm{~mm}$ & $0,67(0,53) a A B$ & $0,48(0,77)$ aA & $1,20(0,28) a A$ \\
\hline & Zirpress & $1,0 \mathrm{~mm}$ & $-0,07(1,02) a A B$ & $-0,36(1,68) a A$ & $-0,50(064) a A$ \\
\hline & \multicolumn{2}{|c|}{ Cimento } & * & $2,64(1,13) a B$ & $0,50(0,33) \mathrm{bA}$ \\
\hline & \multicolumn{2}{|c|}{ Dentes } & $1,04(1,77) \mathrm{aB}$ & $3,38(3,70) b B$ & $-1,67(2,01) \mathrm{cB}$ \\
\hline \multirow{6}{*}{ Rely-X U200 } & \multirow{2}{*}{ E-max } & $0,5 \mathrm{~mm}$ & $1,26(1,50) \mathrm{aA}$ & $1,02(1,18) \mathrm{aA}$ & $0,39(2,56)$ aA \\
\hline & & $1,0 \mathrm{~mm}$ & $0,00(0,75) \mathrm{aA}$ & $0,19(0,55)$ aA & $0,09(0,63) \mathrm{aAB}$ \\
\hline & \multirow{2}{*}{ Zirpress } & $0,5 \mathrm{~mm}$ & $-0,10(2,19) a A$ & $2,00(1,54) b A C$ & $1,20(0,28) a A B$ \\
\hline & & $1,0 \mathrm{~mm}$ & $-0,07(1,02)$ aA & $0,15(0,55) \mathrm{abA}$ & $-0,50(0,64) b A B$ \\
\hline & \multicolumn{2}{|c|}{ Cimento } & * & $29,69(2,26) \mathrm{aB}$ & $0,80(0,22) b A$ \\
\hline & \multicolumn{2}{|c|}{ Dentes } & $1,04(1,77) \mathrm{aA}$ & $3,33(3,70) b C$ & $-1,67(2,01) \mathrm{cB}$ \\
\hline
\end{tabular}

Letras diferentes, minúsculas na linha e maiúsculas na coluna, para cada cimento testado e dentes, indicam diferença estatisticamente significante $(p<0,05)$.

Analisando-se os resultados dos grupos de laminados cimentados com cimento Variolink II, verifica-se que o tipo de envelhecimento não causou alteração de Delta b significativa $(p>0,05)$, independente do sistema cerâmico e da espessura do laminado, com exceção do laminado de 0,5mm com E-max Press, em que houve maior alteração de Delta b após ciclagem, resultado estatisticamente significante $(p<0,05)$ em relação ao grupo envelhecido em água destilada e semelhante $(p>0,05)$ em relação ao grupo Controle. Estes grupos não apresentaram diferença estatisticamente significante $(p>0,05)$ entre si.

Quando o cimento Rely-X U200 foi utilizado para cimentação dos laminados cerâmicos, verificou-se que o sistema cerâmico E-max Press não apresentou diferença estatisticamente significante $(p>0,05)$ em relação ao tipo de envelhecimento. Porém, Zirpress com $0,5 \mathrm{~mm}$ de espessura apresentou diferença estatisticamente significante $(p<0,05)$ após Envelhecimento em água destilada, resultado diferente dos demais grupos, que não apresentaram diferença $(p>0,05)$ entre si. Com 1,0mm, o laminado de Zirpress apresentou alteração significativa $(p<0,05)$ de Delta $b$ no grupo Controle, em relação aos demais grupos, que não apresentaram diferença $(p>0,05)$ entre si. Tanto o grupo de cimento quanto o de dentes manteve a diferença estatisticamente significante $(p<0,05)$ entre os tipos de envelhecimento, independente do tipo de cimento utilizado.

Comparando-se o desempenho dos materiais testados em relação aos envelhecimentos a que foram submetidos, verificou-se que restaurações de E-max Press 
de $0,5 \mathrm{~mm}$ submetidas à Ciclagem obtiveram resultados diferentes estatisticamente $(p<0,05)$ em relação aos dentes e não apresentou diferenças $(p>0,05)$ em relação a nenhum outro grupo, que não apresentaram diferenças $(p>0,05)$ entre si. Amostras cimentadas com RelyX U200 submetidas à Ciclagem não apresentaram diferença estatisticamente significante $(p>0,05)$ nos valores de Delta $b$.

Quando submetidas a Envelhecimento em água destilada, as restaurações cimentadas com Variolink II não apresentaram diferença estatisticamente significante $(p>0,05)$ entre si, mas houve diferença $(p<0,05)$ em relação aos grupos Cimento e Dentes, sem diferença entre si $(p>0,05)$. Comportamento semelhante foi encontrado nos laminados cerâmicos cimentados com Rely-X U200, porém o grupo de Dentes apresentou resultados semelhantes $(p>0,05)$ ao grupo Zirpress $0,5 \mathrm{~mm}$.

Quanto ao grupo Controle, amostras com Variolink II não apresentaram diferenças $(p>0,05)$ entre si, em função do sistema cerâmico e da espessura testada, nem em relação ao cimento testado isoladamente. Porém, houve diferença $(p<0,05)$ de todos os grupos em relação aos Dentes. Quando cimentados com Rely-X U200, houve diferença estatisticamente significante $(p<0,05)$ entre amostras de $E$-max Press de $0,5 \mathrm{~mm}$ em relação ao grupo de dentes, sem diferença $(p>0,05)$ em relação aos demais grupos, que não apresentaram diferenças $(p>0,05)$ entre si. O grupo que utilizou o cimento apresentou diferença significante $(p<0,05)$ em relação ao grupo de Dentes.

O gráfico 5 mostra a alteração de Delta b (cromaticidade no eixo azul-amarelo) dos materiais e cimentos testados, além do grupo de dentes, em relação aos testes realizados para cada sistema cerâmico. 
Gráfico 5- Alteração de Delta b dos grupos estudados.

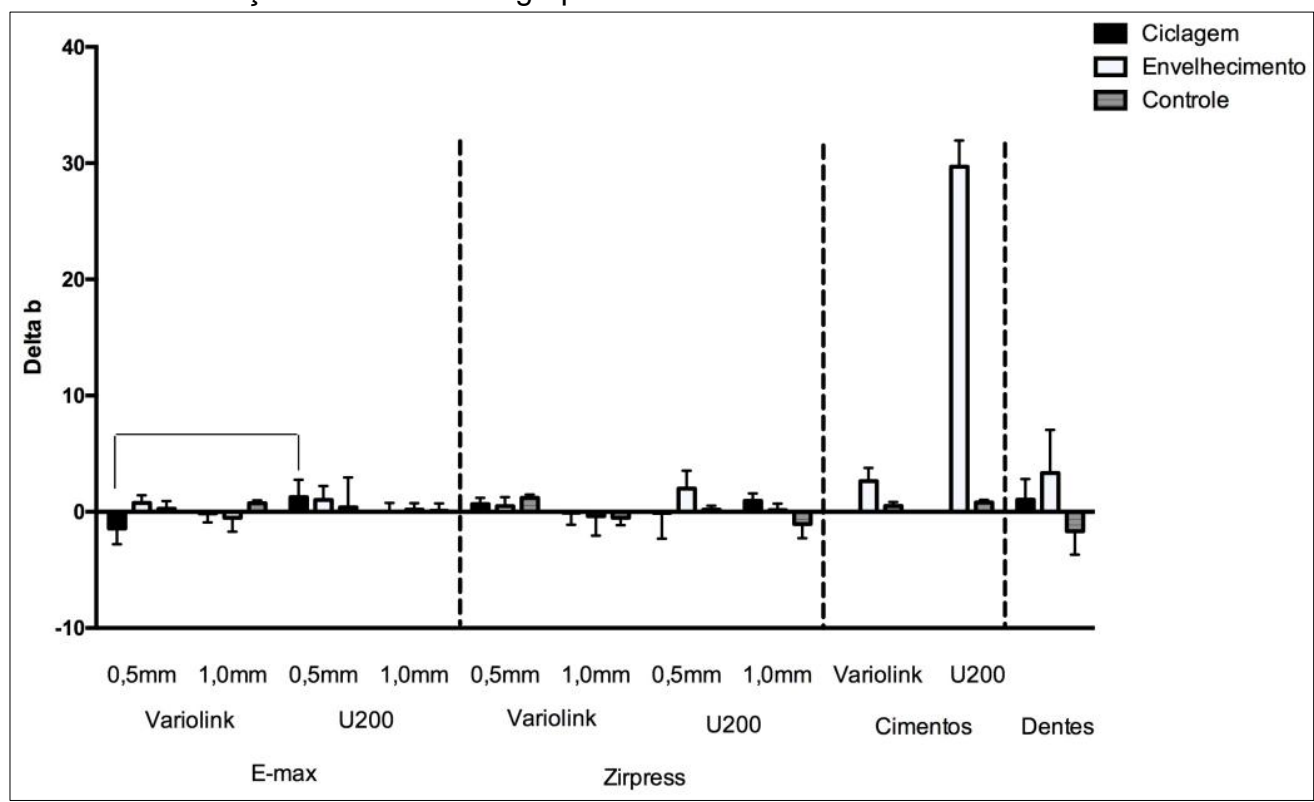

Barras ligadas por linhas indicam diferença estatisticamente significante $(p<0,05)$.

Comparando os resultados para os cimentos testados, é possível observar diferença estatisticamente significante $(p<0,05)$ somente para o grupo E-max Press na espessura de 0,5mm após ciclagem. Os outros grupos não apresentaram diferença estatisticamente significante $(p>0,05)$ em relação ao agente cimentante.

\subsection{Alteração de Translucidez}

Os resultados para alteração de cor podem ser vistos na Tabela 7 e Gráfico 6.

A Tabela 7 mostra a comparação das médias e desvios padrão (3-way ANOVA, Tukey, $\quad p<0,05)$ da alteração de translucidez para cada tipo de cimento testado, comparando os sistemas cerâmicos, as espessuras dos laminados e o tipo de envelhecimento a que foram submetidos. 
Tabela 7- Comparação das médias e desvio padrão (3-way ANOVA, teste de Tukey, $p<0,05$ ) da variação de Translucidez dos grupos estudados.

\begin{tabular}{|c|c|c|c|c|c|}
\hline \multirow{7}{*}{ Variolink II } & \multirow{3}{*}{ E-max } & & Ciclagem & Envelhecimento & Controle \\
\hline & & $0,5 \mathrm{~mm}$ & $-0,04(0,30) a A$ & $0,04(0,54) a A B$ & $0,03(0,33) a A$ \\
\hline & & $1,0 \mathrm{~mm}$ & $-0,05(0,29) a A$ & $0,54(0,38) a A$ & $0,01(0,27) \mathrm{aA}$ \\
\hline & \multirow{2}{*}{ Zirpress } & $0,5 \mathrm{~mm}$ & $0,07(0,38) a A$ & $0,04(0,19) \mathrm{aAB}$ & $0,26(1,17) \mathrm{aA}$ \\
\hline & & $1,0 \mathrm{~mm}$ & $0,29(0,37) a A$ & $-0,44(0,39) a B$ & $0,04(0,09)$ aA \\
\hline & \multicolumn{2}{|c|}{ Cimento } & * & $-1,90(0,97) \mathrm{aC}$ & $-0,44(0,41) b A$ \\
\hline & \multicolumn{2}{|c|}{ Dentes } & $0,46(1,33) a A$ & $-0,55(1,48) b B$ & $-0,20(1,04) a b A$ \\
\hline \multirow{6}{*}{ Rely-X U200 } & \multirow{2}{*}{ E-max } & $0,5 \mathrm{~mm}$ & $0,09(0,31) a A$ & $-0,19(0,43) a A B$ & $-1,21(2,96) b A$ \\
\hline & & $1,0 \mathrm{~mm}$ & $0,02(0,42) a A$ & $0,24(0,26) a A$ & $0,21(0,46) \mathrm{aB}$ \\
\hline & \multirow{2}{*}{ Zirpress } & $0,5 \mathrm{~mm}$ & $0,48(0,46) a A$ & $0,11(0,29) \mathrm{aAB}$ & $0,05(0,13) a B$ \\
\hline & & $1,0 \mathrm{~mm}$ & $0,09(0,26) \mathrm{aA}$ & $0,00(0,18)$ aAB & $0,00(0,08) a B$ \\
\hline & \multicolumn{2}{|c|}{ Cimento } & * & $-1,04(0,57) a B$ & $-0,51(0,51)$ aAB \\
\hline & \multicolumn{2}{|c|}{ Dentes } & $0,46(1,33) \mathrm{aA}$ & $-0,55(1,48) b A$ & $-0,20(1,04) a b A B$ \\
\hline
\end{tabular}

Letras diferentes, minúsculas na linha e maíusculas na coluna, para cada cimento testado e dentes, indicam diferença estatisticamente significante $(p<0,05)$.

Comparando os tratamentos realizados para cada tipo de sistema cerâmico e espessura de restauração, verificou-se que não houve diferença estatisticamente significante $(p>0,05)$ para nenhum grupo de restaurações cimentadas com Variolink II. As diferenças $(p<0,05)$, com esse tipo de cimento, ocorreram entre as amostras de cimento e dentes, sendo que, nesse último grupo, amostras submetidas à Ciclagem apresentaram alteração de translucidez diferente $(p<0,05)$ dos grupos submetidos a Envelhecimento em água destilada. O grupo Controle apresentou resultados semelhantes $(p>0,05)$ a todos os grupos.

Quanto ao cimento Rely-X U200, a maior alteração de translucidez ocorreu com cerâmica E-max Press de 0,5mm no grupo Controle, resultado estatisticamente diferente $(p<0,05)$ dos demais tipos de envelhecimento, que não apresentaram diferenças $(p>0,05)$ entre si. Outra diferença significante ocorreu no grupo de Dentes. Amostras submetidas à Ciclagem apresentaram diferença estatisticamente significante $(p<0,05)$ em relação ao grupo envelhecido em água destilada e ambos não apresentaram diferença $(p>0,05)$ em relação ao grupo Controle. Todos os demais grupos não apresentaram diferença estatisticamente significante $(p>0,05)$ em função do tipo de envelhecimento a que foram submetidos.

Analisando-se o efeito de cada envelhecimento sobre os diferentes sistemas cerâmicos e espessuras de laminados cerâmicos, cimentados com cada um dos cimentos 
resinosos, verificou-se que amostras submetidas à Ciclagem não apresentaram diferença estatisticamente significante $(p>0,05)$ em nenhum dos grupos. $O$ mesmo ocorreu para amostras do grupo Variolink II submetidas a envelhecimento Controle. Quando utilizado o cimento Rely-X U200 com esse tipo de envelhecimento, houve diferença estatisticamente significante $(p<0,05)$ entre amostras de $0,5 \mathrm{~mm}$ de $E$-max Press e todos os demais grupos restaurados com laminados, que não apresentaram diferença significante $(p>0,05)$ entre si. Também não houve diferença $(p>0,05)$ dos grupos restaurados com os grupos Cimentos e Dentes, semelhantes $(p>0,05)$ entre si.

Quando submetidas a Envelhecimento em água destilada, amostras de Zirpress de 1,0mm cimentadas com Variolink II apresentaram diferença estatisticamente significante $(p<0,05)$ de laminados de E-max Press com 1,0mm. Este grupo, por sua vez, apresentou diferença $(p<0,05)$ em relação ao grupo Dentes, que não apresentou diferença $(p>0,05)$ em relação a nenhum outro grupo, a não ser o grupo obtido com o cimento isoladamente, que apresentou diferença estatisticamente significante $(p<0,05)$ em relação a todos os grupos com Variolink II.

Quando analisados os resultados com Rely-X U200, verificou-se diferença estatisticamente significante $(p>0,05)$ entre o grupo E-max Press de $1,0 \mathrm{~mm}$ e amostras de cimentos; estes apresentaram resultados diferentes $(p<0,05)$ do grupo Dentes. Para todas as outras comparações, não houve diferença estatisticamente significante $(p>0,05)$.

O gráfico 6 mostra a alteração de translucidez dos materiais e cimentos testados, além do grupo de dentes, em relação aos testes realizados para cada sistema cerâmico. 
Gráfico 6- Alteração de translucidez dos grupos estudados.

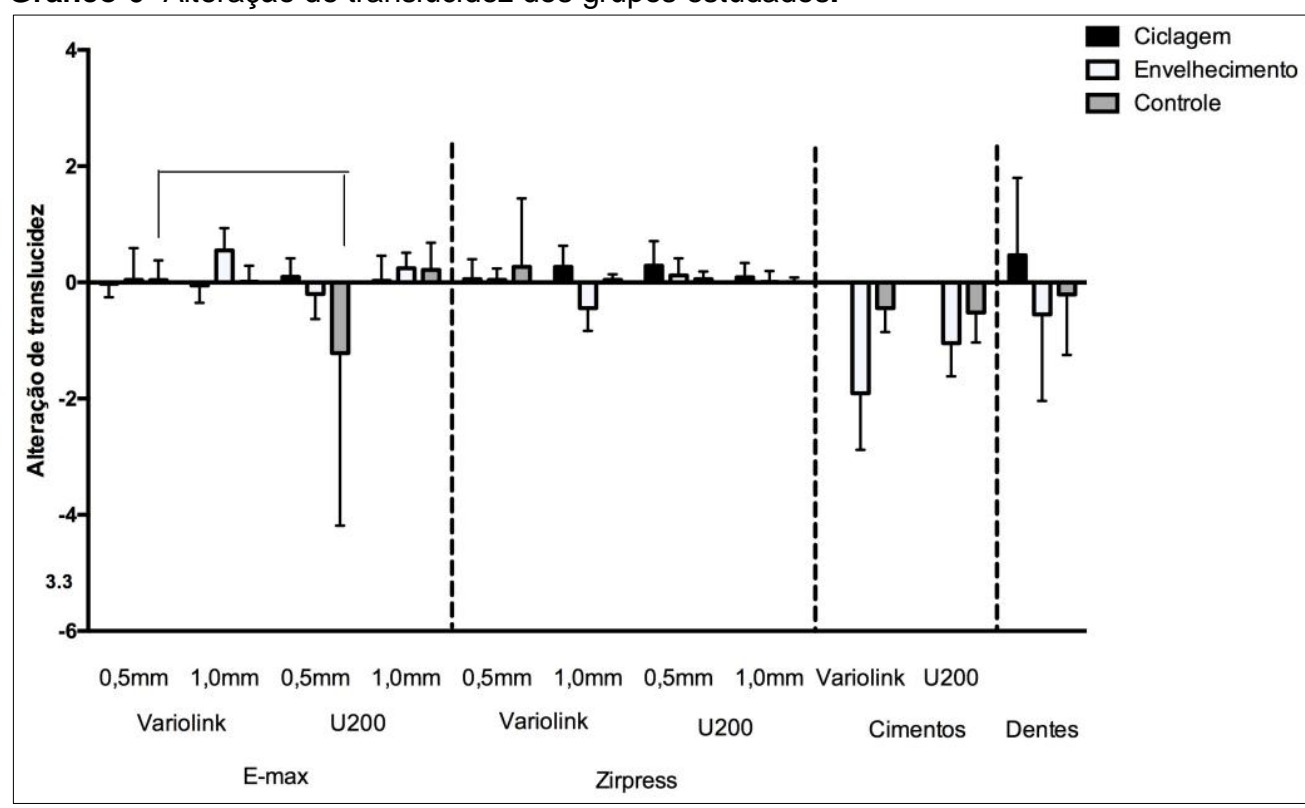

Barras ligadas por linhas indicam diferença estatisticamente significante $(p<0,05)$.

Comparando os resultados para os cimentos testados, é possível verificar uma maior perda na translucidez do grupo E-max Press com espessura de 0,5mm quando utilizado o cimento Rely-X U200 no Grupo Controle, com diferença estaticamente significante $(p<0,05)$ entre os dois. Nos demais grupos não houve diferença estatisticamente significante $(p>0,05)$.

\subsection{Linha de Cimentação}

A Tabela 8 mostra a comparação das médias e desvio padrão (3-way ANOVA, teste de Tukey, $p<0,05)$ dos valores de espessura da linha de cimentação produzida pelos cimentos resinosos para cada cerâmica, nas diferentes espessuras.

Tabela 8- Comparação das médias e desvio padrão (3-way ANOVA, teste de Tukey, $p<0,05$ ) da linha de cimentação $(\mu \mathrm{m})$ dos cimentos resinosos dos grupos estudados.

\begin{tabular}{|c|c|c|c|c|c|}
\hline & & & & & \\
\hline \multirow{3}{*}{ Variolink II } & \multirow{2}{*}{ E-max } & $0,5 \mathrm{~mm}$ & $66,22(18,30) a A$ & $72,11(24,24)$ aA & $46,66(11,05) \mathrm{aA}$ \\
\hline & & $1,0 \mathrm{~mm}$ & $41,55(6,85) \mathrm{aA}$ & $51,55(31,96) \mathrm{aA}$ & $24,33(7,26) \mathrm{aA}$ \\
\hline & Zirpress & $\begin{array}{l}0,5 \mathrm{~mm} \\
1,0 \mathrm{~mm}\end{array}$ & $\begin{array}{l}40,77(15,38) \text { aA } \\
49,44(20,00) \text { aA }\end{array}$ & $\begin{array}{c}70,33(11,15) \mathrm{aA} \\
47,00(3,84) \mathrm{aA}\end{array}$ & $\begin{array}{l}48,11(14,00) \text { aA } \\
56,33(18,77) \text { aA }\end{array}$ \\
\hline \multirow{3}{*}{$\begin{array}{l}\text { Rely-X } \\
\text { U200 }\end{array}$} & \multirow{2}{*}{ E-max } & $0,5 \mathrm{~mm}$ & $76,22(60,94)$ aA & $40,11(3,56)$ aA & $58,88(30,50)$ aA \\
\hline & & $1,0 \mathrm{~mm}$ & $59,88(21,55)$ aA & $38,88(6,76) a A$ & $33,77(6,04) \mathrm{aA}$ \\
\hline & Zirpress & $\begin{array}{l}0,5 \mathrm{~mm} \\
1,0 \mathrm{~mm}\end{array}$ & $\begin{array}{l}47,88(7,12) \text { aA } \\
66,00(6,43) \text { aA }\end{array}$ & $\begin{array}{l}85,88(27,19) \text { aA } \\
53,77(16,64) \text { aA }\end{array}$ & $\begin{array}{l}70,00(16,67) \mathrm{aA} \\
44,88(15,81) \mathrm{aA}\end{array}$ \\
\hline
\end{tabular}

Letras diferentes, maiúsculas na coluna e minúsculas na linha, indicam resultados estatisticamente significantes (3-way ANOVA, Tukey, $p<0,05)$. 
Os sistemas cerâmicos, as diferentes espessuras dos laminados cerâmicos e o tipo de envelhecimento não influenciaram a espessura da linha de cimentação produzida em ambos os cimentos resinosos, fotoativado e autoadesivo, não havendo diferença estatisticamente significante $(p>0,05)$ entre os grupos.

O gráfico 7 apresenta os valores de espessura da linha de cimentação produzidos pelos cimentos fotoativado e autoadesivo.

Gráfico 7- Comparação dos valores de espessura da linha de cimentação dos cimentos estudados.

\section{Linha de Cimentação}

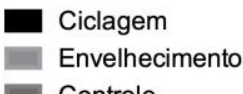

Controle

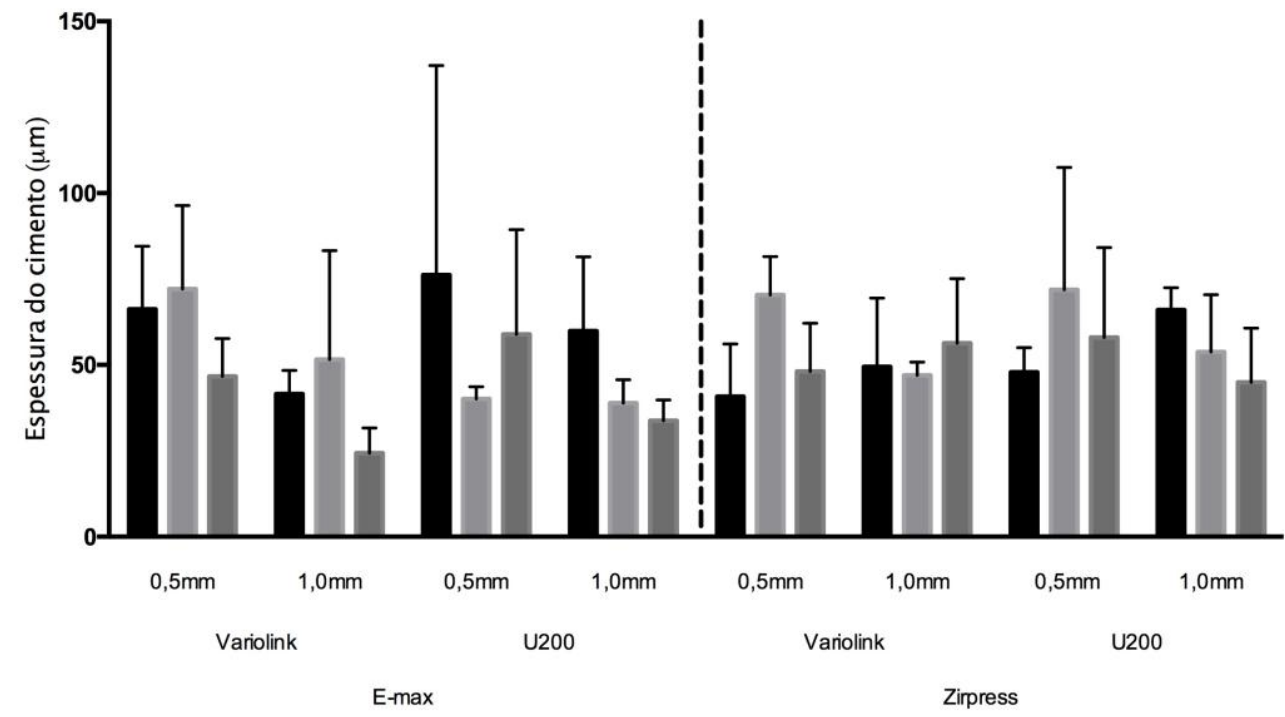

De acordo com os valores apresentados da linha de cimentação, produzida pelos cimentos estudados, não houve diferença estatisticamente significante $(p>0,05)$ entre os cimentos resinosos fotoativado e dual. 

5. Discussãa 



\section{DISCUSSÃO}

O objetivo desse estudo foi avaliar grau de conversão, estabilidade de cor e translucidez de laminados cerâmicos em diferentes espessuras cimentados com dois tipos de cimentos resinosos submetidos a diferentes protocolos de envelhecimento. Partiu-se de 3 hipóteses: 1) Não haveria diferença no grau de conversão dos cimentos em função das espessuras das restaurações; 2) Não haveria diferença na estabilidade de cor e translucidez das restaurações em função do agente cimentante e do protocolo de envelhecimento a que foi submetido e 3) Restaurações mais delgadas sofreriam maior influência na alteração de cor e translucidez.

Os resultados do estudo indicam que a primeira hipótese pode ser totalmente aceita, pois a grau de conversão dos cimentos resinosos não variou em função da espessura da restauração. A segunda hipótese pode ser parcialmente aceita, pois houve alteração de cor e translucidez de restaurações em função do agente cimentante e do protocolo de envelhecimento. A terceira hipótese pode ser parcialmente aceita, pois restaurações de $0,5 \mathrm{~mm}$ apresentaram resultados semelhantes $(p>0,05)$ às de $1,0 \mathrm{~mm}$. As exceções ocorreram quando restaurações de E-maxpress foram cimentadas com Rely-X U200. A alteração de cor significante $(p<0,05)$ ocorreu quando as amostras foram submetidas a Envelhecimento em água destilada e a alteração de translucidez quando submetidas ao grupo Controle.

Neste estudo, foram utilizados dois tipos de cimentos resinosos para cimentação de laminados cerâmicos, um adesivo e fotoativado e outro autoadesivo e dual. O grau de conversão analisada por espectrofotômetro de infravermelho transformado por Fourier (FTIR), utilizado neste estudo, é um método usado para analisar a quantidade dos monômeros convertidos em polímeros de alguns materiais odontológicos, dentre eles os cimentos resinosos (Passos et al, 2013). O grau de conversão dos cimentos resinosos depende do tipo de cerâmica, microestrutura e espessura da cerâmica sobre o cimento (Soares et al, 2006; Lee et al, 2008), que pode alterar a quantidade de luz que é transmitida 
ao cimento resinoso através da restauração durante a fotopolimerização (Heffernan et al, 2002), além do sistema de ativação do cimento resinoso.

Para verificar a influência da espessura e do tipo de cerâmica no grau de conversão dos cimentos resinosos, a quantidade de luz absorvida pelo laminado cerâmico foi previamente determinada através de uma esfera integradora (Illumia, Labsphere, North Sutton, EUA), obtida pela diferença de potência entre a luz que sai do fotopolimerizador e a luz que efetivamente chega ao cimento resinoso. Essa analise mostrou que, apesar de menor quantidade de luz chegar ao cimento resinoso através do laminado cerâmico mais espesso (gráfico 1), essa diferença não foi capaz de influenciar o grau de conversão desses cimentos; ou seja, a quantidade de luz que chegou ao cimento resinoso foi suficiente para a conversão dos monômeros.

Isso pode ser explicado pela influência e modulação do grau de conversão, que está relacionada com a densidade de potência, comprimento de onda e tempo de exposição da fonte luminosa (Spinell et al, 2009). A irradiância total do fotopolimerizador usado neste estudo foi de $1423 \mathrm{~mW} / \mathrm{cm}^{2}$, ou seja, uma alta irradiância, de forma que mesmo que somente $30 \%$ da luz passe através da restauração, é o suficiente para a conversão de monômeros da matriz polimérica. A irradiância sugerida para obter uma polimerização eficaz do cimento resinoso, deve ser superior a $400 \mathrm{~mW} / \mathrm{cm}^{2}$. O protocolo de polimerização tem um efeito crítico no grau de conversão e é um fator que influencia o desempenho do material (Pereira et al, 2010), de forma que quanto maior a densidade de energia aplicada sobre o material resinoso, maior é o grau de conversão (Puppin-Rontani et al, 2012). Segundo Zhang \& Wang (2011) quanto mais espessa a restauração, maior tempo e densidade de potência serão necessários para uma correta polimerização do cimento.

Como os fatores coloração e técnicas de confecção da cerâmica podem influenciar a translucidez das cerâmicas, que por sua vez impactam no grau de conversão dos cimentos (Baldissara et al, 2010; Zhang \& Wang et al, 2011; kilinc et al, 2011), esses fatores foram padronizados no presente estudo, no qual foram usados somente cerâmicas prensadas na mesma cor e translucidez (A1 HT). Os resultados mostram que o tipo de 
cerâmica foi um fator significante no grau de conversão dos cimentos resinosos, quando obtidas restaurações de $1 \mathrm{~mm}$, de forma que a cerâmica a base de zircônia apresentou maior grau de conversão para o cimento autoadesivo/dual.

Isso pode ser explicado pela diferença de translucidez inerente ao próprio material. A translucidez da cerâmica a base de dissilicato de lítio é maior devido à menor quantidade cristalina em comparação com a cerâmica a base de zircônia (Baldissara et al, 2010); assim observa-se um maior grau de conversão para o cimento dual quando utilizada a cerâmica a base de zircônia na espessura de $1 \mathrm{~mm}$, o qual além da polimerização pela luz tem a polimerização química em comparação com o cimento fotoativado que apresenta apenas a polimerização por luz, o que confirma a sua dependência da radiação (Hooshmand et al, 2009).

Neste estudo "in vitro" optou-se pela utilização de dentes bovinos, pois a quantidade de dentes necessária para o estudo (260) era inviável dentro dos aspectos éticos caso optássemos por dentes humanos. Entretanto, os dentes bovinos apresentam propriedades estruturais e ópticas semelhantes aos dentes humanos (Pecho et al, 2012). O substrato escolhido para cimentação dos laminados cerâmicos foi a dentina, uma vez que o preparo para facetas laminadas somente em esmalte é muito restrito na clínica, sendo possível apenas em casos bem específicos. Uma das principais indicações para o tratamento com facetas laminadas é paciente portador de múltiplas restaurações em resina composta que sofrem descoloração e desarmonizam o sorriso. A remoção dessas restaurações para o tratamento sem dúvida irá expor a dentina. Além disso, a dentina é a melhor indicação do cimento autoadesivo (Rely-X U200) conforme utilizado no estudo (Hitz et al, 2012).

Sem dúvida, a cerâmica é o melhor material para confecção dos laminados cerâmicos, devido suas propriedades ópticas muito semelhantes aos dentes (Radz, 2011).

Diferentes sistemas cerâmicos estão indicados para obtenção da restauração laminada em cerâmica, como a cerâmica sinterizada, a cerâmica injetada e prensada e ainda a fresada pelo sistema CAD/CAM. A estrutura e cor da cerâmica podem ser influenciadas pelo técnico durante a confecção das peças em cerâmica sinterizada, uma 
vez que a manipulação e dosagem do pó e líquido podem influenciar a estrutura final da cerâmica (Bahannan et al, 2016). Sendo assim, a experiência e habilidade do técnico interferem no resultado final da restauração (Bahannan et al, 2016). Por isso, neste estudo optou-se por usar a cerâmica injetada e prensada, eliminando a distorção na cor que poderia ser causada pelo próprio operador.

Quanto à composição química da cerâmica, o dentista deve ter conhecimento necessário dos diferentes tipos de cerâmicas e suas características para melhor seleção de material em cada caso específico. A literatura apresenta indicações de material para facetas laminadas de acordo com a necessidade estética e funcional do paciente (FonsFont et al, 2006): Tipo 1A-facetas que não serão expostas a carga funcional e dentes sem alteração de cor; 1B-facetas não serão expostas a carga funcional e dentes com alteração de cor; 2 facetas serão expostas a carga funcional. Para o tipo $1 \mathrm{~A}$ é indicado o uso de cerâmicas feldspáticas ou reforçadas por dissilicato de lítio e leucita. No tipo 1B são indicadas cerâmicas e cimentos com alto grau de opacidade, como a base de óxido de alumínio e zircônia para mascarar a cor do substrato (Fons-Font et al, 2006). No entanto, nas cerâmicas a base de zircônia é esperada uma diminuição da translucidez e consequentemente um maior comprometimento estético no resultado final da restauração (Harata et al, 2016). Para superar essa desvantagem e melhorar a estética das restaurações protéticas, as empresas têm lançado no mercado, cerâmicas a base de zircônia com diferentes graus de translucidez, variando a quantidade de conteúdo cristalino da cerâmica e composição (Manual Zirpress, 2009).

A estabilidade de cor foi analisada no laminado cerâmico sobre o cimento e sobre dentina, porque a alteração de uma ou ambas as estruturas poderiam interferir na cor da restauração final (Stevenson \& Ibbetson, 2010; Chang et al, 2009). Assim, a fim de isolar a variável responsável pela alteração de cor, foram incluídos no design do estudo dois grupos controles: um só com o substrato dental, em que os dentes foram aplainados e as leituras de cor foram realizadas sem qualquer laminado ou cimento sobre o substrato; e por amostras de cimento. 
Os grupos controles também foram submetidos aos diferentes tipos de envelhecimento. O grupo controle dos Cimentos não resistiu ao ensaio de ciclagem termomecânica, fraturando todos os corpos-de-prova. A fratura se deu por conta da fina espessura do corpo-de-prova $(0,5 \mathrm{~mm})$ e a ausência de proteção do cimento pelo laminado cerâmico. Clinicamente, a união do cimento resinoso com a cerâmica diminui as forças de impactos sofridos durante a mastigação (Gurel, 2007). Além disso, a dentina também é resiliente (Gurel, 2007) e colabora para minimizar o dano.

Considera-se, porém, que clinicamente a espessura do cimento é muito menor que a testada, tendo no máximo $120 \mu \mathrm{m}$ (Ukon et al, 2004; Freire et al, 2016). A espessura de 0,5mm para amostras do grupo controle dos Cimentos foi escolhida, pois, apesar de ser bem maior do que a linha de cimentação produzida pelo cimento durante a cimentação dos laminados cerâmicos, essa seria a espessura mínima capaz de permitir colher resultados confiáveis para analise da cor das amostras (Silami et al, 2016; Kilinc et al, 2011).

Com intuito de se determinar de forma precisa as propriedades ópticas dos materiais restauradores, como cor, opacidade e brilho, espectrofotômetros têm sido utilizados para mensuração desses parâmetros. Estes equipamentos permitem adequada interpretação dos resultados (Molenaar et al, 1999), apresentando melhor desempenho quando comparados à avaliação visual (Wee et al, 2002). Porém, a mensuração da cor em espectrofotômetros pode diferir conforme as configurações dos instrumentos (Wee et al, 2002).

Seghi et al. (1989a) analisaram instrumentos auxiliares no processo de escolha da cor de uma restauração e concluíram que os resultados que tinham melhor relação com a percepção visual foram aqueles fornecidos pelo sistema CIE Lab, independentemente do aparelho testado. Além disso, colorímetros portáteis facilitaram o trabalho de leitura.

Seghi et al. (1989b) avaliaram espectrofotômetros e colorímetros em relação a avaliação visual por examinadores treinados de cerâmicas translúcidas e concluíram que a análise de cor com instrumentos era uma importante ferramenta na avaliação de cor de cerâmicas odontológicas e que o desenvolvimento de espectrofotômetros para aplicação na 
clínica odontológica como instrumento auxiliar na escolha da cor de restaurações dentais era necessário.

Estudos mais recentes indicam que os espectrofotômetros são capazes de acertar de forma mais precisa a cor, com a mínima diferença de cor, quando comparada a mensuração de cor pelo método visual por estudantes e clínicos (Bahannan, 2014; Alsalch et al, 2012) auxiliando, dessa forma, seleção de cor na clínica diária.

A espessura média de um laminado cerâmico é $0,7 \mathrm{~mm}$ (Radz et al, 2011). No entanto, nem sempre é possível obter laminados em espessuras exatas na clínica. Por isso, é importante conhecer a interação do cimento com diferentes espessuras da restauração. Dessa forma, para o presente estudo foram selecionadas espessuras de 0,5 e 1,0mm.

O diâmetro do corpo-de-prova do laminado utilizado foi de $6 \mathrm{~mm}$, não sendo necessário a confecção de guias de padronização para leituras de cor, uma vez que a ponteira do espectrofotômetro usado tem o mesmo diâmetro, e assim era possível a colocação da ponteira centralizada no corpo-de-prova sempre na mesma posição, como realizado em estudos anteriores (Dozic et al, 2003; Bahannan et al, 2016), não comprometendo a leitura da cor, uma vez que a parte iluminante da ponteira é menor $(5 \mathrm{~mm})$.

Como limites de aceitabilidade de alteração de cor foram usados os seguintes índices (Silami et al, 2016; Garcia et al, 2013):

- $\Delta \mathrm{E}<1$ - Alteração de cor não detectada pelo olho humano;

- $\Delta \mathrm{E} \leq 3,3$ - Alteração de cor detectada pelo olho humano, mas considerada aceitável;

- $\Delta \mathrm{E}>3,3$ - Alteração de cor detectada pelo olho humano e considerada clinicamente inaceitável, sendo indicada a substituição do material restaurador por motivos estéticos.

A alteração de cor dos grupos Cimento foi maior que dos grupos restaurados no envelhecimento com água, com índices acima do clinicamente aceitável $(\Delta \mathrm{E}>3,3)$. Quando o cimento não está protegido pelo dente e cerâmica, toda a área de cimento e substrato é 
exposta homogeneamente ao método de envelhecimento, potencializando seus efeitos, justificando os altos valores de alteração de cor e perda de translucidez dos grupos controle dos Cimentos. Os dentes também apresentaram altos índices de alteração de cor, acima do clinicamente aceitável $(\Delta \mathrm{E}>3,3)$ para o envelhecimento em água e ciclagem termomecânica devido à ausência de proteção pelo laminado cerâmico e ao manchamento íntrinseco e extrínseco sofrido pelo dente (Hattab et al, 1999) durante o envelhecimento.

Nota-se que o método de envelhecimento não foi um fator significante na alteração de cor das restaurações cimentadas com o cimento fotoativado Variolink II. Para o cimento autoadesivo/dual Rely-X U200 o envelhecimento com água produziu maior alteração de cor nas cerâmicas a base de zircônia em ambas as espessuras em comparação com a ciclagem termomecânica e controle.

Os cimentos resinosos convencionais, como o Variolink II, apresentam valores mais baixos de sorção de água e solubilidade que os cimentos autoadesivos, como o Rely-X U200 (Liu et al, 2011; Petropoulou et al, 2015). Os cimentos resinosos autoadesivos contêm grupos ácidos, tais como carboxílicos e fosfatados na sua composição, que são os monômeros funcionais responsáveis pela adesão do cimento à estrutura dentária. Esses monômeros são sensivelmente mais hidrofílicos que os monômeros BisGMA e TEGDMA presentes na composição dos cimentos adesivos (Petropoulou et al, 2015), justificando a ausência do efeito do envelhecimento com água destilada nos grupos cimentados com o cimento convencional Variolink II e a mudança de cor em alguns grupos restaurados com o cimento autoadesivo Rely-X U200, independente da espessura. Esses achados são diferentes dos encontrados em estudos que utilizaram o envelhecimento artificial acelerado (Silami et al, 2016; Oliveira et al, 2015). Este método, porém, produz alterações de cor muito mais significativas aos materiais a base de resina, acima do limite clinicamente aceitável, devido à presença da luz UV-B que é absorvida por aminas, que estão na composição do material, criando moléculas com elevada energia. Essas moléculas ativas podem reagir com o oxigênio ou com outros grupos aromáticos e aumentar a absorção de luz, especialmente na região verde-azul do espectro eletromagnético resultando numa 
mudança de cor para o amarelo e vermelho das amostras (Ruyter et al, 1987; Oliveira et al 2015).

Quando comparados os cimentos, o cimento Rely-X U200 produziu maior alteração de cor nas restaurações com E-maxPress de $0,5 \mathrm{~mm}$ do que o cimento Variolink II. Isso pode ser justificado, pois o cimento Rely-X U200 é dual e sua descoloração está relacionada à oxidação da amina terciária presente na sua composição (Lu \& Power, 2004; Almeida et al, 2015). Os cimentos resinosos de cura dual contêm distintas aminas, uma reage com o peróxido benzoíla (polimerização química) e outro com a canforoquinona (polimerização pela luz, física). A amina do sistema redox (polimerização química) é aromática e mais propensa à degradação. A amina do sistema polimerizado pela luz é alifática e mais estável quimicamente, tendendo a causar menos variação de cor (Akgungor et al, 2005; Almeida et al, 2015).

A translucidez da restauração é importante para a mimetização dos dentes naturais, uma vez que as estruturas mineralizadas dos dentes apresentam translucidez inerente à sua estrutura biológica, sendo necessário reproduzir essa característica (Lee et al, 2007). No entanto, a translucidez do sistema cerâmico, pode sofrer influência do cimento resinoso, como demonstram os resultados obtidos para o laminado E-maxpress de $0,5 \mathrm{~mm}$ quando cimentado com cimento Rely-X U200 em comparação com o cimento Variolink II. Isso é justificado pelo fato da cerâmica a base de zircônia ser menos translúcida que a cerâmica a base de dissilicato de lítio (Baldissara et al, 2010), pois a translucidez é proporcional à composição das cerâmicas, sendo que as com maior conteúdo cristalino apresentam menor translucidez (Antonson \& Anusavice, 2011). Dessa forma a influência do cimento resinoso é maior na cerâmica a base de dissilicato de lítio.

A menor espessura também contribuiu para que essa influência fosse percebida. $O$ cimento Rely-X U200 apresentou variação negativa de Delta b (tom mais "amarelado") depois dos testes a que foram submetidos (tabela 6), o que pode ter influenciado na translucidez do material cerâmico. 
Outro fato que pode ter influenciado a perda de translucidez das amostras de Emax Press de $0,5 \mathrm{~mm}$ foi a maior alteração de Delta a (Tabela 5) quando cimentado com cimento Rely-X U200, no grupo controle, com tendência à predominância do tom vermelho.

É possível notar resultados homogêneos da translucidez nos grupos submetidos à ciclagem, independente da cerâmica e espessura utilizada. Isto pode ser explicado pela composição da cerâmica Zirpress, que possui fluorapatita prensada com oxido de zircônio. Esses cristais estão presentes em diferentes tamanhos (Manual Zirpress, 2009) resultando na otimização das propriedades ópticas como a translucidez. A fim de aumentar a translucidez da zircônia, poros e impurezas devem ser removidas, pois eles criam diferentes índices de refração que leva a dispersão óptica com redução da translucidez (Yamashita et al 2011; Harada et al, 2016). A alumina que aumenta as propriedades mecânicas da zircônia é a impureza mais comum (Harada et al, 2016).

Alguns estudos corroboram os resultados encontrados, quando afirmam que a melhoria das propriedades do pó e a modificação dos seus métodos de prensagem podem aumentar a translucidez da zircônia (Yamashita et al, 2011, Harada et al, 2016). Entretanto, o tamanho da partícula e a temperatura de sinterização também influenciam na translucidez da zircônia (Zhang, 2014; Harada et al, 2016). Quanto maior a temperatura de sinterização, maior os tamanhos dos grãos e, consequentemente, maior translucidez terá a cerâmica (Harada et al, 2016) pois quando a luz passa através da zircônia ela pode dispersar nos limites de grão. Quando o tamanho do grão é menor acorrerá maior dispersão pela maior quantidade de grãos (Harada et al, 2016). Neste estudo a temperatura de sinterização utilizada foi de $910^{\circ} \mathrm{C}$. Geralmente, a temperatura de sinterização das zircônias convencionais fica em torno de 1200 à $1350^{\circ} \mathrm{C}$. Assim, a alta translucidez da zircônia pode requerer temperaturas mais altas de sinterização para obter grãos maiores (Harad et al, 2016).

A adaptação marginal, descrita por Holmes em 1989, como o preenchimento do gap presente entre a parede interna da restauração e a parede externa do preparo deve medir em torno de 25 a $40 \mu \mathrm{m}$ de espessura, espaço suficiente para o escoamento do agente 
cimentante. Os valores de linha de cimentação mensurados neste estudo, variaram de $24 \mu \mathrm{m}$ à $85 \mu \mathrm{m}$, sem diferença entre os cimentos resinoso utilizados na pesquisa. Para cimentação realizada com cimentos resinosos esses valores são considerados "clinicamente aceitáveis", podendo a linha de cimentação medir até 120um (Ukon et al, 2004, Freire et al, 2016).

Os protocolos de envelhecimento em água destilada e ciclagem não foram capazes de degradar o cimento presente na linha de cimentação. Isso pode ser explicado pela proteção do laminado cerâmico sobre o cimento e pela capacidade de se aderirem tanto ao dente quanto à restauração (Manso et al, 2011), além da menor solubilidade do cimento resinoso (Pegoraro et al, 2007) em comparação a outros cimentos.

Estudos mostram que fatores como método de confecção das restaurações cerâmicas, a configuração da margem da coroa, o espaço do cimento e a técnica de cimentação podem levar a diferentes resultados de adaptação ou linha de cimentação $(\mathrm{Ng}$ et al, 2014; Reich et al, 2008). Não obstante, os resultados encontrados no presente estudo demonstram que apesar das variáveis testadas (cimento resinoso e técnica de cimentação), a linha de cimentação apresentou-se semelhante em todos os grupos. Isso pode ser explicado pela padronização do procedimento de cimentação utilizada no estudo, através do uso da haste de um delineador e a carga de 380 gramas, viabilizando a técnica e eliminando a interferência humana em estudos in vitro (Silami et al, 2016). Dessa forma, assegurou-se que as alterações que poderiam ocorrer no estudo seriam advindas de outros fatores que não a espessura do cimento.

Depois de adequadamente cimentado, o laminado cerâmico torna-se parte integral da estrutura do dente e sofre parte das tensões de carga aplicadas durante o ciclo mastigatório. O cimento é submetido às diversas forças mastigatórias, ciclos térmicos, e influenciado pelo efeito hidrolítico da água e diferentes químicas presentes na boca (Cotert et al, 2009; Aboushelib et al, 2012). A ciclagem termomecânica simula a mastigação, e de acordo com os resultados encontrados, os cimentos são eficientes na estabilidade de cor de restaurações ao longo do tempo, pois não houve diferença na alteração de cor e 
translucidez nos grupos restaurados quando usada a ciclagem termomecânica como um método de envelhecimento.

A simplificação da cimentação com cimentos autoadesivos, visando melhorar a sensibilidade técnica é uma inquestionável conquista. No entanto, não deve ser mais importante do que a manutenção da cor em tratamentos estéticos. Sendo assim, é sempre importante aliar características do cimento e do material restaurador, com vistas nos melhores resultados em longo prazo.

Devemos considerar também que a cimentação depende do tratamento da peça para uma adequada adesão do cimento ao laminado cerâmico. Estudos têm investigado a influência de diferentes tipos de tratamento da superfície na rugosidade (Kara et al, 2012) e resistência de união (Barghi et al, 2000; Yavuz et al, 2013). A alteração da rugosidade pode alterar as propriedades ópticas dos dentes, entre elas a translucidez. Considerando-se que restaurações cada vez mais finas têm sido utilizadas em tratamentos estéticos para harmonizações do sorriso é importante avaliar a influência do tratamento de superfície nas propriedades ópticas de diferentes cerâmicas cimentadas com cimentos simplificados ou não. 

6. Conclusãa 



\section{CONCLUSÃo}

Considerando-se os resultados do estudo foi possível concluir que:

1. Laminados cerâmicos com maior espessura não diminuíram o grau de conversão dos cimentos resinosos;

2. O cimento dual obteve maior grau de conversão que o cimento fotoativado quando utilizado sob o sistema Zirpress;

3. A linha de cimentação produzida pelos dois cimentos foi semelhante e não interferiu nas propriedades ópticas dos sistemas cerâmicos estudados;

4. A ciclagem termomecânica não foi significante para alterações de cor e translucidez dos sistemas cerâmicos cimentados com cimentos fotoativado e dual/autoadesivo;

5. O tipo de cimento resinoso influencia a estabilidade de cor dos sistemas cerâmicos quando submetido a envelhecimento em água destilada;

6. Os protocolos de envelhecimento (ciclagem termomecânica e envelhecimento em água destilada) alteraram a cor e a translucidez dos substratos e dos cimentos;

7. A alteração de cor dos sistemas cerâmicos, em função do envelhecimento, ocorreu devido à diminuição da coordenada $L$ para ambos os métodos testados. As alterações nas demais coordenada seguiram direções de cromaticidade segundo o método de envelhecimento utilizado. 

Referências 



\section{REFERÊNCIAS}

Aboushelib MN, Elmahy WA, Ghazy MH. Internal adaptation, marginal accuracy and microleakage of a pressable versus a machinable ceramic laminate veneers. J Dent. 2012;40(8):670-7.

Aguiar TR, Francescantonio MD, Arrais CAG, Ambrosano GMB, Davanzo C, Giannini M. Infuence of curing mode and time on degree of conversion of one conventionla and two selfahesive resin cements. Operative Dentistry. 2010;35(3):295-299.

Akgungor G, Akkayan B, Gaucher H. Influence of ceramic thickness and polymerization mode of a resin luting agent on early bond strength and durability with a lithium disilicatebased ceramic system. J Prosthetic Dent. 2005;94:234-41.

Algahtani MQ, Aljurais RM, Alshaafi MM. The effects of different shades of resin luting cement on color of ceramic veneers. Dent Mater J. 2012;31:354-361.

Almeida JR, Schmitt GU, Kaizer MR, Boscato N, Moraes RR. Resin-based luting agents and color stability of bonded ceramic veneers. J Prosthethic Dent. 2015;114(2):272-7.

AISaleh S, Labban M, AlHariri M, et al: Evaluation of self shade matching ability of dental students using visual and instrumental means. J Dent 2012;40s:e82-e87

Antonson SA, Anusavice KJ. Contrast ratio of veneering and core ceramics as a function of thickness. In J Prosthodont. 2011;14:316-320.

Azer SS, Ayash GM, Johnston WM, Khalil MF, Rosentiel SF. Effect of esthetic core shades on the final color of IPS Empress all-ceramic crowns. J Prosthetic Dent. 2006 Dec;96:397401.

Baldissara P, Llukacej A, Ciocca L Valandro FL, Scotti R. Translucency of zircônia copings made with diferente CAD/CAM systems. J Prosthetic Dent. 2010;104:6-12.

Bagis B, Turgut S. Optical properties of current ceramics systems for laminate venners. J Dent. 2013 Aug;41Suppl 3:e24-30.

Bahannan SA: Shade matching quality among dental students using visual and instrumental methods. J Dent 2014;42:48-52

Barghi N, McAlister E. Porcelain for veneers. J Esthet Dent. 1998;10(4):191-7. 
Braga RR, Ballester RY, Daronch M. Influence of time and adhesive system on the extrusion shear strength between feldspathic porcelain and bovine dentin. Dent Mater 2000;16:303-10.

Calamia JR. Etched porcelain facial veneers: a new treatment modality based on scientific and clinical evidence. N Y J Dent. 1983 Sep-Oct; 53(6):255-9.

Chang J, Da Silva JD, Sakai M, Kristiansen J, Ishikawa-Nagai S. The optical effect of composite luting cement on all ceramic crowns. J Dent. 2009 Dec;37:937-43.

CIE (Commission Internationale de I'Eclairage).Colorimetry technical report.CIE Pub. №15, 2nd ed. Vienna, Austria:Bureau Central da La CIE, 1986, [corrected reprint 1986].

Cantoro A, Coracci C, Conigli I, Magni E, Polimeni A, Ferrari M. Influence of ultrasound application on inlays luting with self-adhesive resin cements. Clin Oral Invest. 2011;15:61723.

Corciolani G, Vichi A, Louca C, Ferrari M. Influence of layering thickness on the color parameters of a ceramic system. Dent Mater. 2010 Aug; 26(8):737-42.

Crisp S, Abel G, Wilson AD. The quantitative measurement of the opacity of aesthetic dental filling materials. J Am Dent Assoc. 1979; 58:1585-96.

DeBoever, J.A.; Mccall, W.D.; Holden, S.; Ash, M.M. Functional occlusal forces: an investigation by telemetry. J Prosthet Dent; 1978;40:326-33.

Della-Bona A. Characterizing ceramics and the interfacial adhesion to resin: II- The relationship of surface treatment, bond strength, interfacial toughnesss and fractografy. $J$ Appl Oral Sci. 2005 Jun;13(1):1-9

Della Bona A, Anusavice JK. Microstructure, composition and eitching topography of dental ceramics. Int. J. Prosthodont. 2002;15(2):159-67.

Devigus A, Lombardi G. Shading Vita In-ceram YZ substructures: influence on value and chroma, part II. Int J Comput Dent. 2004 Oct; 7(4):379-88.

Devigus A, Lombardi G. Shading Vita YZ substructures: influence on value and chroma, part I. Int J Comput Dent. 2004 Jul; 7(3):293-301. 
De Munck J, Vargas M, Van Landuyt K, Hikita K, Lambrechts P,Van Meerbeek B. Bonding of an auto-adhesive luting material to enamel and dentin. Dent Mater. 2004 Dec; 20(10):963-71.

Dozic A, Kleverlaan J, Meegdes M, van der Zel J, Feilzer AJ. The influence of porcelain layer thickness on the final shade of ceramic restorations. J Prosthetic Dent. 2003 Dec;90:563-70.

Dozic A, Tsagkari M, Khashayar G, Aboushelib M. Color management of porcelain veneers: influence of dentin and resin cement colors. Quintessence Int. 2010 Jul-Aug; 41(7):567-73.

Dumfahrt $H$, Schäffer $H$. Porcelain laminate veneers. A retrospective evaluation after 1 to 10 years of service: Part II--Clinical results. Int J Prosthodont. 2000 Jan-Feb; 13(1):9-18.

Feire A, Archegas LR. Porcelain laminate venner on a highty discolored tooth: a case report. Journal of Canadian Dental Association. 2010;76:126-7.

Fontijn-Tekamp, F.A.; Slagter, A.P.; Van der Bilt, A.; et al. Biting and chewing in overdentures, full dentures, and natural dentitions.J Dent Res. 2010;.79:1519-1524.

Fons-Font A, solá-Ruíz M, Granell-Ruíz M, labaig-Rueda C, Martínez-Gonzáles A. Choice of ceramic for use in treatment with porcelain laminate veneers. Med Oral Patol Cir Bucal. 2006;11(3):E297-302.

Friedman MJ. A 15-year review of porcelain veneer failure--a clinician's observations. CompendContinEducDent. 1998 Jun; 19(6):625-8.

Garcia LaF, Mundim FM, Pires-de-Souza FdeC, Puppin Rontani RM, Consani S. Effect of artificial accelerated aging on the optical properties and monomeric conversion of composites used after expiration date. Gen Dent 2013;61:1-5.

Guada GB, Gonçalves LS, Correr AB, Moraes RR, Sinhoreti MAC, Correr-sobrinho L. Luting glass ceramic restorations using a self-adhesive resin cement under different dentin conditions. J Appl Oral Sci.2010;18;244-248.

Gürel G. Porcelain laminate veneers: minimal tooth preparation by design. Dent Clin North Am. 2007 Apr;51(2):419-31.

Ham L, Okamoto A, Fukushima M, Okiji T. Evaluation of physical properties and surface degradation of self-adhesive resin cements. Dental Mater J. 2007;26(6);906-12. 
Haselton DR, Diaz-Arnold AM, Hillis SL. Clinical assessment of high-strength all-ceramic crowns. J Prosthetic Dent. 2000 Apr;83(4):396-401.

Hattab FN, Qudeimat MA, al-Rimawi HS. Dental discoloration: an overview. J Esthetic Dent. 1999;11(6):291-310.

Heffernan MJ, Aquilino SA, Diaz-Arnold AM, Haselton DR, Stanford SM, Vargas MA. Relative translucency of six all-ceramic system.Part I: Core Materials. J Prosthet Dent 2002a; 88(1):4-9.

Heffernan MJ, Aquilino SA, Diaz-Arnold AM, Haselton DR, Stanford SM, Vargas MA. Relative translucency of six all-ceramic system.Part II: Core and venner materials. J Prosthet Dent 2002b; 88(1):10-15.

Hekimoglu C, Anil N, Etikan I. Effect of accelerated aging on the color stability of cemented laminate veneers. Int J Prosthodont. 2000 Jan-Feb; 13(1):29-33.

Homes JR, Bayne SC, Holland GA, Sulik WD. Considerations in measurement of marginal fit. J Prosthetic Dent.1992;67(5):594-9.

Hoodshmand T, Mahmoodi N, keshvad A. Microhardness of a resin cement polymerized by light-emiting diode and halogen lights through ceramic. J Prosthodont. 2009;18:411-416.

International Organization for Standardization. ISO no. 4049:2000, Dentistry-polymer-based filling, restorative and luting materials.

Janda R, Roulet JF, Latta M, Steffin G, Ruttermann S. Color stability of resin-based filling materials after aging when cured with plasma or halogen light. Eur J Oral Sc. 2005 Jun;113:251-7.

Jonhston WM, Reisbick MH. Color and translucency changes during and after curing of esthetic restorative materials. Dent Mater. 1997 Mar;13(2):89-97.

Kelly JR, Nishimura I, Campbell SD. Ceramics in dentistry: historical roots and current perspectives. Journal of Prosthetic Dentistry. 1996;75:18-32.

Kilinc E, Antonson SA, Hardigam PC, Kesercioglu A. Resin cement color stability and its influence on the final shade of all-ceramics. J Dent 2011;39:30-36. 
Kilinc E, Antonson SA, Hardigam PC, Kesercioglu A. The effect of ceramic restoration shade and thickness on the polymerization of light and dual-cured resin cements. Operative Dentistry. 2011;36(6):661-669.

Komine F, Tomic M, Strub JR. Influence of different adhesive resin cements the fractura strength of aluminum oxide ceramic posterior crows. J Prosthet Dent. 2004 Oct;92(4):35964.

Lambrechts P, Willems G, Vanherle G, Braem M. Aesthetic limits of light-cured composite resins in anterior teeth. Int Dent J. 1990 Jun;40(3):149-58.

Lee IB, An W, Chang J, Um CM. Influence of ceramic thickness and curing mode on the polymerization shrinkage kinetics of dual-cured resin cement. Dent Mater. 2008 Aug;24(8):1141-7.

Lee YK, Cha HS, Ahn JS.Layered color of all-ceramic core and veneer ceramics. J Prosthetic Dent. 2007 May;97:279-86.

LeeYK. Comparasion of CIELAB Delta $E\left({ }^{*}\right)$ and CIEDE2000 color-differences after polymerization and thermocycling of resin composites. Dent Mater. 2005;21(7):678-82.

Lee Y.K, Lim BS, Kim CW. Difference in the colour and colour change of dental resin composites by the background. J Oral Rehabil. 2005 Mar;32:227-233.

Liu Q, meng X, Yoshiba K, Luo X. Bond degradation behavior of self-adhesive cement and convencional resin cements bonded to silanized ceramic. J prosthetic Dent. 2011;105:17784.

Lu H, Powers JM. Color stability of resin cements after accelerated aging. American JournalofDentistry. 2004;17:354-8.

Luo XP, Zhang L. Effect of venner in techniqueson color and translucency of Y-TZP. JournalofProsthodontics. 2010;10:465-70.

Luo MR, Gui G, Rigg B. The development of the CIE 2000 colour-diffrence formula: CIEDE2000. Color Research and Application. 20001;26(5):340-50.

Manual técnico IPS e-max Press e IPS e-maxZirPress, Ivoclar/vivadent, 2009.

Magne P, Known KR,Besler C, Hodges JS, Douglas WH. J Prosthet Dent 1999;81:327-34. 
Manso AP, Silva NR, Bonfante EA, Pegoraro TA, Dias RA, Carvalho RM. Cements and adhesives for all-ceramic restorations. Dent Clin North Am. 2011;55(2):311-32.

McLean JW. The science and art of dental ceramics.Oper Dent. 1991;16(4):149-56.

Meijering AC, Roeters FJ, Mulder J, Creugers NH. Patients' satisfaction with different types of veneer restorations. J Dent. 1997 Nov; 25(6):493-7.

Melo Freire CA, Borges GA, caldas D, Santos RS, Ignácio AS, Mazur RF. Marginal and quality of interfaces in lithium disilicate crows-Influence of manufacturing and cementation techniques. Oper Dent. 2016: Nov 1 [Epub ahead of print]

Milleding P, Haag P, Neroth B, Renz I. Two years of clinical experience with Procera titanium crowns. Int JProsthodont.1998 May-Jun; 11:224-32.

Molenaar R, Ten Bosch JJ, Zijp JR. Determination of Kubelka-Munk scattering and absorption coefficients by diffuse illumination.Appl Opt. 1999 Apr; 38(10):2068-77

Miyagawa Y, Powers JM, O'Brien WJ. Optical properties of direct restorative materials.J Dent Res. 1981;60:890-4

Nakamura T, Wakabayashi K, Kinuta S, Nishida H, Yatani M.H, Mechanical properties of new self-adhesive resin-based cement. Journal of Prosthodontic Research. 2010;54:59-64.

$\mathrm{Ng}$ J, Ruse D, Wyatt C. A comparison of the marginal fit of crowns fabricated with digital and conventional methods. Journal of Prosthetic Dentistry.2014:112(3):555-60.

Oliveira DC, Ayres AO, Rocha MG, PUppin Rontani RM, Ferracane JL, Sinhoreti MA. Effect of diferente in vitro aging methods on color stability of a dental resin-based composite using CIELAB and CIEDE2000 color-diffrence fromulas. 2015;27(5):322-30.

Ozturk N, Usumez A, Usumez S, Ozturk B. Degree of conversion and surfasse hardness od resin cement cured with different curing units. Quintessence Int 2005;36:771-777.

Pavan S., Berger S.,.Bedran-Russo A. K. B., The effect of dentin pretreatment on the microtensile bond strength of self-adhesive resin cements. The Journal of Prosthetic Dentistry. 2010;104(4):258-264.

Passos SP, Kimpara ET, Bottino MA,Santos GC Jr, Rizkalla AS. Effect of ceramic shade on the degree of conversion of a dual-cure resin cement analyzed by FTIR.Dent Mater.2013 Mar;29(3):317-23. 
Pecho OE,GhineaR, lonescu AM, CardonaJde L, Paravina RD, Pérez Mdel M. Color and translucency of zirconia ceramics, human dentine and bovine dentine. J Dent. 2012 Dec;40 Suppl 2:e34-40.

Pegoraro TA, Da Siva NRFA, Carvalho RM. Cements for use in Esthetic Dentistry. Dent Clin North Am. 2007;51(2):453-71.

Pereira SG, Fulgencio R, Nune TG, Toledano M, Osorio R, Carvalho RM. Effect of curing protocol on the polymerization of dual cured resin cements. Dental Materials. 2010;26:71018

Perez Mdel M, Saleh A, Yebra A, Pulgar R. Study of the variation between CIELAB delta E* and CIEDE2000 color-differences of resin composites. Dent Mater J. 2007 Jan;26(1):21-8.

Petropoulou A, Vrochari AD, Hellwig E, Stampf S, Polydorou O. Water sorpion and water solubility of self-etching and self-adhesive resin cements. $J$ Prosthethic Dent. 2015;115(5):674-9.

Pires-de-Souza FdeC, Casemiro LA, Garcia Lda F, Cruvinel DR. Color stability of dental ceramics submitted to artificial accelerated aging after repeated firings. J Prosthetic Dent. 2009 Jan; 101(1):13-8.

Puppin-Ronatani RM, Dineli Rg, de Paula AB, Fucio SBP, Ambrosano GMB, Pascon FM. Indepth polymerization of a self-adhesive dual-cured resin cement. Operative Dent. 2012;37(2):188-194.

Radz GM. Minimum thickness anterior porcelain restorations. Dent Clin North Am 2011;55:353-370.

Reich S, Gozdowski S, Trentzsch L, Frankenberger R, Lohbauer U. Marginal fit of heatpressed vs.CAD/CAM processed all-ceramic onlays using a milling unit propttype. Operative Dentistry. 2008;33(6):644-50.

Saker S, Alhzzawi A, Ozcan M. Adhesive strength of self-adhesive resins to lithium disilicate ceramic and dentin: effect of dentin chelanting agents. Odontology. 2016 Jan;104(1):53-9.

Schanda JD. Colorimetry. In: DeCusatis C. Handbook of applied photometry. 2 ed. New York: Optical Society of America Springer-Verlag; 1998. p. 327-412. 
Seghi RR, Hewlett ER, Kim J. Visual and instrumental colorimetric assessments of small color differences on translucent dental porcelain. J Dent Res. 1989 Dec; 68(12):1760-4.

Seghi RR, Johnston WM, O'Brien WJ. Performance assessment of colorimetric devices on dental porcelains. J Dent Res. 1989 Dec; 68(12):1755-9.

Seghi RR, Johnston WM, O'Brien WJ. Spectrophotometric analysis of color differences between porcelain systems.J Prosthetic Dent.1986 Jul;56:35-40.

Shahin R, Kern M. Effect on fair-abrasion on the retention of zircônia ceramic crows luted with diferente cements before and after artificial aging. Dent Mater. 2010 Sep;26(9):922-8.

Silami FDJ, Tonani R, Alandia-Roman CC, Pires-de-Souza FdeCP. Influence of diferente types of resin luting agents on color stability of ceramic laminate veneers subjected to accelerated artificial aging. Braz Dent J. 2016;27(1):95-100.

Sjögren G, Lantto R, Tillberg A. Clinical evaluation of all-ceramic crowns (Dicor) in general practice. J Prosthet Dent 1999 Mar; 81(3):277-84.

Stevenson B, Ibbetson R. The effect of the substructure on the colour of samples/restorations veneered with ceramic: A literature review. J Dent. 2010 May; 38:361368.

Soares Cj, Silva NR, Fonseca RB. Influence of the feldspsthic ceramic thickness and shade on the microhardness of dual resin cement. Oper Dent. 2006 May-Jun;31(3):384-9.

Sorensen JA, Cruz M, Mito WT, Raffliner O, Meredith HR, Foser HP.A clinical investigation o three-unit fixed partial dentures fabricated with a lithium disilicate glass-ceramic. Pratical Procedures and Aesthetic Dentistry.1999;11:95-106.

Spinell T, Schedle A, Watts DC. Polymerization shrinkage kinetics of dimethacrylate resincements. Dent Mater 2009;25:1058-66.

Tung FF,Goldstein Gr, Jang S, Hittelman E. The repeatability of an intraoral dental colorimeter. J Prosthet Dent 2002;88:585-90.

Turgut S, Bagis B. Color stability of laminate venner materials. Journal of Prosthetic Dentistry. 2011;39:57-64.

Vichi A, Ferrari M, Davidson CL. Influence of ceramic and cement thickness on the masking of various types of opaque posts. J Prosthetic Dent. 2000 Apr;83:412-417. 
Vichi A, Fraioli A, Davidson CL, Ferrari M. Influence of thickness on color in multi-layering technique. Dent Mater. 2007 Dec; 23(12):1584-9.

Xing W, Jiang T, Ma X, Liang S, Wang Z, Sa Y, Wang Y. Evaluation of the esthetic effect of resin cements and try-in pastes on ceramic veneers.J Dent. 2010 May; 38(s2):87-94.

Walter R, Miguez PA, Pereira PN. Microtensile bond strength of luting materials to coronal and root dentin. J EsthetRestor Dent. 2005 May; 17(3):165-71.

Wang F, Takahashi $\mathrm{H}$, Iwasaki N. Translucency of dental ceramics with different thicknesses. J Prosthet Dent. 2013 Jul;110(1):14-20.

Wee AG, Monaghan P, Johnston WM. Variation in color between intended matched shade and fabricated shade of dental porcelain. J Prosthetic Dent. 2002 Jun; 87(6):657-66.

Wyszwcki G, Stiles WS. Color science: concepts and methods, quantitative data and formulae. 2 ed. New York: John Wiley\& Sons; 1982 p. 112-23.

Ukon S, Ishikawa M, Tohyama M, Sato H. Determination of the fabricating conditions for the preferable marginal and internal adaptation of the mica crystal castable ceramic crown. Dent Mater J 2004;23(1):53-62.

Zahng Y, Griggs JA, Benham BS. Influence of powder/liquid mixing radio on porosity and translucency of dental porcelains. J Prosthet Dent 2004;91:128-35.

Zhang $X$ \& Wang F. Hardness of resin cement cured under different thickness of lithium disilicate-based ceramic. Chin Med J. 2011; 124(22):3762-3767. 

Apêndices 

Os quadros 1 ao 11 constam as leituras iniciais de cor, sobre fundo preto e fundo branco, dos corpos de prova que foram submetidos a envelhecimento em água destilada.

Quadro I- Leituras de cor das amostras de E-max Press na espessura de 0,5mm cimentados com cimento fotoativado.

\begin{tabular}{|c|c|c|c|c|c|c|c|c|c|c|c|c|c|c|c|c|c|c|c|}
\hline \multicolumn{20}{|c|}{ E-max Press 0,5mm Variolink II } \\
\hline \multirow{2}{*}{$\begin{array}{c}\text { Corpo de } \\
\text { Prova }\end{array}$} & \multirow{2}{*}{ EIXO } & \multicolumn{4}{|c|}{ LEITURA INICIA Branco } & \multicolumn{4}{|c|}{ LEITURA INICIAL Preto } & \multicolumn{4}{|c|}{ LEITURA Final Branco } & \multicolumn{4}{|c|}{ LEITURA FINAL Preto } & \multicolumn{2}{|c|}{$\Delta$} \\
\hline & & LI1 & $\mathrm{LI} 2$ & LI3 & MEDIA LI & LF1 & LF2 & LF3 & MEDIA LF & LI1 & LI2 & LI3 & MEDIA LI & LF1 & LF2 & LF3 & MEDIA LF & $\Delta \mathrm{E}$ & $\Delta T$ \\
\hline \multirow{3}{*}{. } & $\mathbf{L}$ & 95,3 & 95,6 & 95,2 & 95,36 & 95,3 & 95,6 & 95,6 & 95,5 & 91,6 & 92,4 & 92 & 92 & 93 & 92,2 & 93,10 & 92,77 & \multirow{3}{*}{3,62} & \multirow{3}{*}{0,43} \\
\hline & $\mathbf{a}$ & $-1,6$ & $-1,6$ & $-1,6$ & $-1,6$ & $-1,6$ & $-1,6$ & $-1,4$ & \begin{tabular}{|l|}
$-1,53$ \\
\end{tabular} & $-0,7$ & $-0,8$ & $-0,7$ & -0.73 & $\begin{array}{l}-0,8 \\
\end{array}$ & $-0,7$ & $-0,8$ & $\begin{array}{l}-0,77 \\
\end{array}$ & & \\
\hline & b & 19,1 & 18,8 & 18,6 & 18,83 & 18,7 & 18,8 & 19,9 & 19,13 & 19,9 & 19,8 & 19,8 & 19,83 & 19,9 & 20 & $-0,77$ & 19,87 & & \\
\hline \multirow{3}{*}{2} & L & 91 & 91,1 & 90,9 & 91 & 90,9 & 90,7 & 91,2 & 90,93 & 89 & 89,7 & 88,9 & 89,2 & 89,3 & 89,3 & 88,8 & 89,13 & \multirow{3}{*}{2,14} & \multirow{3}{*}{0,15} \\
\hline & $a$ & $-1,8$ & $-1,8$ & $-1,8$ & $-1,8$ & $-1,8$ & $-1,8$ & $-1,7$ & $-1,76$ & -1 & $-0,9$ & -1 & $-0,97$ & $-1,3$ & -1 & -1 & $-1,10$ & & \\
\hline & b & 15,9 & 16 & 15,9 & 15,93 & 15,8 & 15,7 & 16,1 & 15,86 & 16,7 & 17 & 16,5 & 16,73 & 16,3 & 16,7 & 16,6 & 16,53 & & \\
\hline \multirow{3}{*}{3} & L & 98,4 & 98,4 & 98,4 & 98,4 & 98,5 & 98,5 & 98,4 & 98,46 & 95,1 & 95,2 & 95,2 & 95,17 & 95,2 & 95,3 & 95,4 & 95,3 & \multirow{3}{*}{3,50} & \multirow{3}{*}{0,26} \\
\hline & $\mathbf{a}$ & $-0,7$ & $-0,6$ & $-0,7$ & $-0,66$ & $-0,7$ & 0,7 & $-0,7$ & $-0,7$ & 0,4 & 0,5 & 0,5 & 0,47 & 0,6 & 0,6 & 0,6 & 0,6 & & \\
\hline & b & 21,6 & 21,6 & 21,2 & 21,46 & 21,2 & 21,2 & 21,5 & 21,3 & 21,9 & 22,2 & 22,2 & 95,17 & 22,4 & 22,6 & 22,7 & 22,57 & & \\
\hline \multirow{3}{*}{4} & $\mathbf{L}$ & 98,7 & 98,3 & 98,3 & 98,43 & 98,7 & 99,5 & 99,5 & 99,23 & 95,2 & 95,3 & 95,4 & 95,3 & 95,3 & 95,4 & 95,3 & 95,33 & \multirow{3}{*}{3,17} & \multirow{3}{*}{$-0,83$} \\
\hline & $a$ & $-0,8$ & $-0,8$ & $-0,7$ & $-0,76$ & $-0,9$ & $-0,9$ & $-0,9$ & $-0,9$ & $-0,4$ & $-0,2$ & $-0,2$ & $-0,27$ & $-0,2$ & $-0,2$ & $-0,2$ & $-0,20$ & & \\
\hline & b & 21,1 & 21,2 & 21,1 & 21,13 & 20,5 & 20,5 & 20,6 & 20,53 & 20,8 & 21,3 & 21,4 & 21,17 & 21,3 & 21,4 & 21,3 & 21,33 & & \\
\hline \multirow{3}{*}{5} & $\mathbf{L}$ & 95,9 & 95,9 & 96,1 & 95,96 & 95,7 & 95,8 & 95,8 & 95,76 & 94,3 & 93,8 & 94 & 94,03 & 94,1 & 93,7 & 94,1 & 93,97 & \multirow{3}{*}{2,38} & \multirow{3}{*}{$-0,14$} \\
\hline & $\mathbf{a}$ & $-0,8$ & $-0,9$ & -1 & $-0,9$ & -1 & -1 & -1 & -1 & 0,20 & 0,20 & 0,20 & 0,20 & 0,2 & 0,2 & 0,3 & 0,23 & & \\
\hline & b & 20,3 & 19,8 & 19,7 & 19,93 & 19,9 & 19,9 & 19,9 & 19,9 & 20,9 & 20,9 & 20,5 & 20,77 & 20,4 & 20,6 & 21,2 & 20,73 & & \\
\hline & L & 95,5 & 95,6 & 95,9 & 95,66 & 95,8 & 95,7 & 95,8 & 95,76 & 95,1 & 92,4 & 92,8 & 93,43 & 92,5 & 92,3 & 92,7 & 92,5 & & \\
\hline 6 & $\mathbf{a}$ & $-0,1$ & 0 & $-0,1$ & $-0,66$ & $-0,1$ & $-0,1$ & $-0,1$ & $-0,1$ & 0,80 & 0,90 & 1,10 & 0,93 & 1,1 & 1,1 & 1,1 & 1,10 & 2,45 & 0,77 \\
\hline & b & 22,4 & 22,6 & 22,5 & 22,5 & 22,4 & 22,2 & 22,1 & 22,23 & 22 & 22,8 & 0,93 & 22,67 & 23,2 & 23,2 & 23 & 21,13 & & \\
\hline & $L$ & 93,6 & 94 & 93,5 & 92,13 & 94,2 & 94,8 & 94,6 & 94,53 & 92,1 & 92,1 & 92,4 & 92,2 & 92,2 & 91,9 & 92,5 & 92,2 & & \\
\hline 7 & $\mathbf{a}$ & $-1,1$ & $-1,1$ & $-1,2$ & $-1,13$ & $-1,1$ & $-1,1$ & $-1,1$ & $-1,1$ & $-0,9$ & $-0,9$ & $-0,9$ & $-0,90$ & -1 & $-0,9$ & -1 & $-0,97$ & 1,59 & $-0,84$ \\
\hline & b & 19,4 & 19,7 & 19,5 & 19,53 & 19,6 & 20,1 & 20 & 19,9 & 19,9 & 20,1 & 20 & 20,0 & 19,9 & 20,1 & 20,1 & 20,03 & & \\
\hline & $\mathbf{L}$ & 93,8 & 93,2 & 93,3 & 93,43 & 93,2 & 93,4 & 93,7 & 93,43 & 93,1 & 92,9 & 93,1 & 93,03 & 93,5 & 93,6 & 94 & 93,70 & & \\
\hline 8 & $a$ & $-1,3$ & $-1,3$ & $-1,4$ & $\begin{array}{l}-1,33 \\
\end{array}$ & $-1,4$ & $-1,4$ & $-1,4$ & $-1,4$ & $-0,6$ & $-0,3$ & $-0,4$ & $-0,43$ & $-0,6$ & $-0,5$ & -0.6 & $-0,57$ & 2,23 & 0,54 \\
\hline & b & 15,7 & 15,3 & 15,1 & 15,36 & 15 & 15 & 15,2 & 15,06 & 17 & 17,7 & 17,4 & 17,37 & 16,8 & 17 & 16,8 & 16,87 & & \\
\hline & L & 94,3 & 94,4 & 94,5 & 94,4 & 94,7 & 94,5 & 94,4 & 94,53 & 93,5 & 93,3 & 93,2 & 93,33 & 93,4 & 93,2 & 93,5 & 93,37 & & \\
\hline 9 & $\mathbf{a}$ & $-1,2$ & $-1,1$ & $-1,2$ & $-1,16$ & $-1,2$ & $-1,2$ & $-1,2$ & $-1,2$ & $-0,4$ & $-0,3$ & $-0,4$ & $-0,37$ & $-0,4$ & $-0,4$ & $-0,4$ & $-0,40$ & 2,08 & $-0,19$ \\
\hline & b & 19,8 & 19,9 & 19,9 & 19,86 & 19,6 & 19,6 & 19,6 & 19,6 & 21,6 & 21,3 & 21,5 & 21,47 & 21,5 & 21,6 & 21,6 & 21,57 & & \\
\hline & L & 96,5 & 96,8 & 96,8 & 96,7 & 96,7 & 96,7 & 96,9 & 96,76 & 92,5 & 92 & 92,6 & 92,37 & 92,5 & 92,8 & 92,8 & 92,70 & & \\
\hline 10 & $a$ & $-1,3$ & $-1,3$ & $-1,4$ & $-1,33$ & $-1,3$ & $-1,3$ & $-1,4$ & $-1,33$ & $-0,6$ & $-0,6$ & $-0,7$ & $-0,63$ & $-0,8$ & $-0,8$ & $-0,8$ & $-0,80$ & 4,39 & 0,29 \\
\hline & b & 18,2 & 18,4 & 18,1 & 18,23 & 18,3 & 18,2 & 18 & 18,16 & 18,1 & 18,1 & 18,4 & 18,20 & 18 & 18,1 & 18,2 & 18,10 & & \\
\hline
\end{tabular}


Quadro 2- Leituras de cor das amostras de E-max Press na espessura de 1,0mm cimentados com cimento fotoativado.

\begin{tabular}{|c|c|c|c|c|c|c|c|c|c|c|c|c|c|c|c|c|c|c|c|}
\hline \multicolumn{20}{|c|}{ E-max Press 1,0mm Variolink II } \\
\hline \multirow{2}{*}{$\begin{array}{c}\text { Corpo de } \\
\text { Prova }\end{array}$} & \multirow{2}{*}{ EIXO } & \multicolumn{4}{|c|}{ LEITURA INICIA Branco } & \multicolumn{4}{|c|}{ LEITURA INICIAL Preto } & \multicolumn{4}{|c|}{ LEITURA Final Branco } & \multicolumn{4}{|c|}{ LEITURA FINAL Preto } & \multicolumn{2}{|c|}{$\Delta$} \\
\hline & & LI1 & LI2 & LI3 & MEDIA LI & LF1 & LF2 & LF3 & MEDIA LF & LI1 & LI2 & LI3 & MEDIA LI & LF1 & LF2 & LF3 & MEDIA LF & $\Delta \mathrm{E}$ & $\Delta \mathrm{T}$ \\
\hline \multirow{3}{*}{1} & $\mathbf{L}$ & 89,2 & 89,2 & 89,2 & 89,2 & 91,1 & 91,3 & 91,2 & 89,33 & 88,2 & 88,6 & 89,2 & 88,67 & 89,4 & 90,1 & 89,1 & 89,53 & \multirow{3}{*}{0,64} & \multirow{3}{*}{0,8} \\
\hline & $\mathbf{a}$ & 0,1 & 1 & 0,1 & 0,4 & 0,1 & 0,1 & 0,1 & 0,1 & 0,3 & 0,5 & 0,5 & 0,43 & 0,6 & 0,6 & 0,6 & 0,60 & & \\
\hline & b & 20,9 & 20,9 & 21,1 & 20,96 & 21,1 & 21 & 20,9 & 21 & 20,4 & 21 & 21,2 & 20,87 & 21,2 & 21,5 & 21 & 21,23 & & \\
\hline \multirow{3}{*}{2} & $L$ & 92,8 & 93 & 93,1 & 92,96 & 92,7 & 92,6 & 92,7 & 92,7 & 91,9 & 93,1 & 94,2 & 93,07 & 93,9 & 94,5 & 94,6 & 94,33 & \multirow{3}{*}{1,17} & \multirow{3}{*}{0,98} \\
\hline & $a$ & $-1,7$ & $-1,7$ & $-1,7$ & $-1,7$ & $-1,7$ & $-1,7$ & $-1,7$ & $-1,7$ & $-0,5$ & $-0,7$ & $-0,9$ & $-0,70$ & $-0,8$ & $-0,9$ & $-0,9$ & $-0,87$ & & \\
\hline & b & 16,4 & 16,5 & 16,6 & 16,5 & 16,3 & 16,4 & 16,4 & 16,36 & 17,1 & 17 & 17,2 & 17,10 & 17,2 & 17,1 & 17 & 17,10 & & \\
\hline \multirow{3}{*}{3} & $\mathbf{L}$ & 92,6 & 91,9 & 91,9 & 92,13 & 91,7 & 91,7 & 91,9 & 91,76 & 92 & 92,3 & 92,4 & 92,23 & 92,6 & 94,1 & 94,1 & 93,60 & \multirow{3}{*}{1,04} & \multirow{3}{*}{1} \\
\hline & $a$ & $-0,9$ & $-0,9$ & -1 & $-0,93$ & $-0,9$ & $-0,9$ & -1 & $-0,93$ & $-0,2$ & $-0,2$ & $-0,3$ & $-0,23$ & $-0,3$ & $-0,5$ & $\begin{array}{c}-0,4 \\
\end{array}$ & $-0,40$ & & \\
\hline & b & 19 & 18,9 & 18,8 & 18,9 & 18,1 & 18,9 & 18,9 & 18,63 & 19,5 & 19,6 & 19,9 & 19,67 & 19,6 & 18,9 & 19,1 & 19,220 & & \\
\hline \multirow{3}{*}{4} & $L$ & 91,4 & 91,2 & 91,7 & 91,43 & 92,4 & 91,6 & 91,4 & 91,8 & 89 & 89,6 & 89,9 & 89,5 & 90 & 89,5 & 89,9 & 89,8 & \multirow{3}{*}{2,09} & \multirow{3}{*}{0} \\
\hline & $a$ & $-0,8$ & $-0,8$ & $-0,8$ & $-0,8$ & $-0,8$ & $-0,8$ & $-0,8$ & $-0,8$ & $-0,1$ & $-0,1$ & $-0,2$ & $-0,13$ & $-0,2$ & $-0,1$ & $\begin{array}{l}-0,2 \\
\end{array}$ & $-0,17$ & & \\
\hline & b & 19 & 19,1 & 19,1 & 19,06 & 19,1 & 19,1 & 19 & 19,06 & 18,4 & 18,7 & 18,8 & 18,63 & 18,6 & 18,9 & 19 & 18,83 & & \\
\hline \multirow{3}{*}{5} & $L$ & 94,1 & 93,8 & 93,8 & 93,9 & 93,9 & 93,5 & 93,7 & 93,7 & 90,9 & 90,8 & 91 & 90,9 & 91 & 91,7 & 91,3 & 91,33 & \multirow{3}{*}{3,13} & \multirow{3}{*}{0,32} \\
\hline & $\mathbf{a}$ & -1 & -1 & -1 & -1 & -1 & -1 & -1 & -1 & $-0,6$ & $-0,5$ & $-0,6$ & $-0,57$ & $-0,5$ & $-0,5$ & $-0,5$ & $-0,50$ & & \\
\hline & b & 18,1 & 17,9 & 17,8 & 17,93 & 18 & 18 & 18 & 18 & 16,9 & 17,2 & 17,4 & 17,17 & 17,1 & 17,8 & 17,5 & 17,47 & & \\
\hline & $L$ & 94 & 94,4 & 93,9 & 94,1 & 94,3 & 94,1 & 94,4 & 95,76 & 91,6 & 91,7 & 91,7 & 91,67 & 91,7 & 91,7 & 91,8 & 91,8 & & \\
\hline 6 & $a$ & $-0,4$ & $-0,5$ & $-0,4$ & $-0,43$ & 0,4 & $\begin{array}{l}-0,4 \\
\end{array}$ & $\begin{array}{l}-4 \\
\end{array}$ & $-1,33$ & $-0,3$ & $-0,3$ & $-0,3$ & $-0,30$ & $-0,3$ & $\begin{array}{l}-3 \\
\end{array}$ & $\begin{array}{l}-1,4 \\
\end{array}$ & $-1,23$ & 2,67 & 0,01 \\
\hline & b & 20 & 20,1 & 20,2 & 20,1 & 20,3 & 20,4 & 20,3 & 20,33 & 18,9 & 19,1 & 19 & 19,00 & 19 & 19,2 & 19,3 & 19,17 & & \\
\hline & $\mathbf{L}$ & 94 & 93,9 & 94 & 92,13 & 93,9 & 94 & 94,1 & 94 & 88,1 & 88 & 88,7 & 88,27 & 89,2 & 88,8 & 89,1 & 89,03 & & \\
\hline 7 & $\mathbf{a}$ & $-0,6$ & $-0,6$ & $-0,6$ & $-0,6$ & $-0,7$ & $-0,7$ & $-0,7$ & $-0,7$ & $-1,4$ & $-1,2$ & $-1,2$ & $-1,27$ & $-1,3$ & $-1,1$ & $-1,2$ & $-1,20$ & 6,64 & 0,73 \\
\hline & $b$ & 18,4 & 18,2 & 18,2 & 18,26 & 18,1 & 18,2 & 18,1 & 18,13 & 14,7 & 15 & 15,1 & 14,93 & 15,1 & 15,7 & 15,4 & 15,40 & & \\
\hline & $\mathbf{L}$ & 90,6 & 90,3 & 90,4 & 90,43 & 90,4 & 90,4 & 90,4 & 90,4 & 89,7 & 90,9 & 90,1 & 90,23 & 90 & 90,1 & 90 & 90,03 & & \\
\hline 8 & $a$ & $-1,6$ & $-1,6$ & $-1,6$ & $-1,6$ & $-1,7$ & $-1,7$ & $-1,7$ & $-1,7$ & $-1,2$ & $-1,2$ & $-1,2$ & $-1,20$ & $-1,2$ & $-1,1$ & $-1,2$ & $-1,17$ & 0,83 & 0,18 \\
\hline & b & 14,6 & 14,8 & 14,6 & 14,66 & 14,6 & 14,7 & 14,7 & 14,66 & 15,2 & 15,4 & 15,5 & 15,37 & 15,5 & 15,6 & 15,6 & 15,57 & & \\
\hline & $L$ & 97 & 96,9 & 96,9 & 96,93 & 96,8 & 96,8 & 97,2 & 96,93 & 92,6 & 93,2 & 93,4 & 93,07 & 93,3 & 93,6 & 93,6 & 93,5 & & \\
\hline 9 & $\mathbf{a}$ & $-0,9$ & $-0,8$ & $-0,8$ & $\begin{array}{l}-0,83 \\
\end{array}$ & $-0,8$ & $-0,9$ & $-0,8$ & $-0,83$ & $-0,5$ & $-0,4$ & $-0,3$ & $-0,40$ & $-0,2$ & $-0,2$ & $-0,2$ & $-0,20$ & 3,99 & 0,74 \\
\hline & b & 20,1 & 20,1 & 20,2 & 20,13 & 20,2 & 20,1 & 20,1 & 20,13 & 18,9 & 19,2 & 19,6 & 19,23 & 19,9 & 19,8 & 19,7 & 19,80 & & \\
\hline & $L$ & 93 & 93,1 & 93,2 & 93,1 & 93,1 & 93,1 & 93 & 93,1 & 91,3 & 91,5 & 91,5 & 91,43 & 91,9 & 92,6 & 92 & 92,17 & & \\
\hline 10 & $a$ & $-1,3$ & $-1,2$ & $-1,2$ & $\begin{array}{l}-1,23 \\
\end{array}$ & $-1,3$ & $-1,3$ & $-1,3$ & $\begin{array}{l}-1,3 \\
\end{array}$ & $\begin{array}{l}-09 \\
\end{array}$ & $-0,8$ & $-0,8$ & $\begin{array}{c}-0,83 \\
\end{array}$ & $-0,8$ & $-0,8$ & $-0,8$ & $-0,80$ & 1,79 & 0,71 \\
\hline & b & 17,9 & 18 & 18 & 17,96 & 18 & 17,9 & 17,9 & 17,93 & 17,4 & 17,5 & 17,5 & 17,47 & 17,7 & 17,9 & 17,7 & 17,77 & & \\
\hline
\end{tabular}


Quadro 3- Leituras de cor das amostras de E-max Press na espessura de 0,5mm cimentados com cimento autoadesivo.

\begin{tabular}{|c|c|c|c|c|c|c|c|c|c|c|c|c|c|c|c|c|c|c|c|}
\hline \multicolumn{20}{|c|}{ E-max Press 0,5mm Rely-X U200 } \\
\hline \multirow{2}{*}{$\begin{array}{c}\text { Corpo de } \\
\text { Prova }\end{array}$} & \multirow{2}{*}{ EIXO } & \multicolumn{4}{|c|}{ LEITURA INICIA Branco } & \multicolumn{4}{|c|}{ LEITURA INICIAL Preto } & \multicolumn{4}{|c|}{ LEITURA Final Branco } & \multicolumn{4}{|c|}{ LEITURA FINAL Preto } & \multicolumn{2}{|c|}{$\Delta$} \\
\hline & & LI1 & $\mathrm{LI} 2$ & LI3 & MEDIA LI & LF1 & LF2 & LF3 & MEDIA LF & LI1 & LI2 & LI3 & MEDIA LI & LF1 & LF2 & LF3 & MEDIA LF & $\Delta \mathrm{E}$ & $\Delta T$ \\
\hline \multirow{3}{*}{1} & $\mathbf{L}$ & 94,3 & 94,7 & 95,3 & 94,76 & 95,4 & 95,8 & 95,7 & 95,63 & 91,9 & 91,9 & 91,9 & 91,90 & 92,3 & 93 & 92,9 & 92,73 & \multirow{3}{*}{3,66} & \multirow{3}{*}{$-0,02$} \\
\hline & $\mathbf{A}$ & $-0,9$ & $-0,8$ & $-0,8$ & $-0,83$ & $-0,8$ & $-0,9$ & $-0,9$ & $-0,86$ & $-0,7$ & $-0,6$ & $-0,6$ & $-0,63$ & $-0,6$ & $-0,5$ & $-0,5$ & $-0,53$ & & \\
\hline & B & 20,6 & 20,9 & 21,1 & 20,86 & 21,1 & 21 & 21,1 & 21,1 & 23 & 23,2 & 23,2 & 23,13 & 23,1 & 21,4 & 21,7 & 21,4 & & \\
\hline \multirow{3}{*}{2} & $\mathbf{L}$ & 87,9 & 87,9 & 88,6 & 88,13 & 88,3 & 90,3 & 89,4 & 89,33 & 87,7 & 86,9 & 87,2 & 87,20 & 87 & 86,7 & 87,2 & 86,97 & \multirow{3}{*}{2,05} & \multirow{3}{*}{$-1,01$} \\
\hline & A & $-1,1$ & $-1,2$ & $-1,1$ & $-1,13$ & $-1,2$ & $-0,9$ & $-1,2$ & $-1,1$ & $-0,7$ & $-0,9$ & $-0,8$ & $-0,80$ & $-0,7$ & $-0,7$ & $-0,7$ & $-0,70$ & & \\
\hline & B & 18,4 & 18,1 & 18,8 & 18,43 & 18,1 & 20,2 & 18,5 & 18,93 & 20,8 & 19,7 & 20,2 & 20,23 & 20 & 20 & 20,3 & 20,10 & & \\
\hline \multirow{3}{*}{3} & $\mathbf{L}$ & 91,7 & 91,8 & 92 & 91,83 & 91,9 & 92,3 & 92 & 92,06 & 89,3 & 89,6 & 89,2 & 89,37 & 89,5 & 89,1 & 89,4 & 89,33 & \multirow{3}{*}{3,52} & \multirow{3}{*}{$-0,15$} \\
\hline & A & $-0,7$ & $-0,6$ & $-0,7$ & $-0,66$ & $-0,7$ & 0,8 & $-0,6$ & $-0,7$ & $-0,5$ & $-0,5$ & $-0,5$ & $-0,50$ & $-0,4$ & $-0,5$ & $-0,5$ & $-0,47$ & & \\
\hline & B & 21,3 & 21,1 & 21 & 21,13 & 21,2 & 20,6 & 21,3 & 21,03 & 23,5 & 24 & 23,4 & 23,63 & 23,3 & 23,3 & 23,6 & 23,53 & & \\
\hline \multirow{3}{*}{4} & $\mathbf{L}$ & 95,9 & 95,9 & 96,1 & 95,96 & 96 & 97,2 & 96 & 99,23 & 90,2 & 89,2 & 88,9 & 89,43 & 89,1 & 89,2 & 89,6 & 89,3 & \multirow{3}{*}{6,60} & \multirow{3}{*}{$-0,08$} \\
\hline & A & -1 & -1 & -1 & -1 & -1 & $-1,1$ & -1 & $-1,03$ & -1 & -1 & $-0,9$ & $-0,97$ & $-1,1$ & -1 & -1 & $-1,03$ & & \\
\hline & B & 18,8 & 18,8 & 18,9 & 18,83 & 18,9 & 18,8 & 19,1 & 18,93 & 20,2 & 19,8 & 19,3 & 19,77 & 19,2 & 19,4 & 19,7 & 19,43 & & \\
\hline \multirow{3}{*}{5} & $\mathbf{L}$ & 96,3 & 96,2 & 96,4 & 95,96 & 96,6 & 96,8 & 96,8 & 96,73 & 93,5 & 92,9 & 93,3 & 93,23 & 92,9 & 93 & 93,4 & 93,10 & \multirow{3}{*}{3,09} & \multirow{3}{*}{0,61} \\
\hline & $\mathbf{A}$ & $-0,3$ & $-0,3$ & $\begin{array}{l}-0,3 \\
\end{array}$ & $-0,3$ & $-0,3$ & $-0,4$ & $-0,4$ & $-0,36$ & $-0,6$ & $-0,6$ & $-0,7$ & $\begin{array}{l}-0,63 \\
\end{array}$ & $-0,8$ & $-0,7$ & $-0,6$ & $-0,70$ & & \\
\hline & B & 22,2 & 22,1 & 22,2 & 22,16 & 22,1 & 21,9 & 22 & 22 & 23,9 & 21,6 & 21,3 & 22,27 & 19,2 & 21,1 & 21,3 & 21,20 & & \\
\hline & $\mathbf{L}$ & 96,4 & 96,2 & 96,2 & 96,26 & 96,8 & 96,9 & 97,4 & 97,03 & 94,5 & 94,6 & 94,7 & 94,6 & 94,7 & 94,8 & 95 & 94,83 & & \\
\hline 6 & A & $-0,2$ & $-0,1$ & 0 & $-0,1$ & $-0,1$ & 0 & $-0,1$ & $-0,06$ & 0,2 & 0,2 & 0,4 & 0,27 & 0,1 & 0,1 & 0,2 & 0,13 & 2,29 & $-0,49$ \\
\hline & B & 25,2 & 25,3 & 25,5 & 25,33 & 25,7 & 25,9 & 25,5 & 25,7 & 26,3 & 26,9 & 27,4 & 26,87 & 26,4 & 26,8 & 26,7 & 26,63 & & \\
\hline & $\mathbf{L}$ & 99,7 & 99,9 & 99,9 & 99,83 & 100,6 & 100,2 & 100,7 & 100,5 & 95,4 & 95 & 95,1 & 95,17 & 95,1 & 95 & 95,3 & 95,13 & & \\
\hline 7 & A & $-0,5$ & $-0,6$ & $-0,6$ & $-0,56$ & $-0,7$ & $-0,5$ & $-0,6$ & $-0,6$ & $-0,2$ & $-0,2$ & $-0,2$ & $-0,20$ & $-0,3$ & $-0,2$ & $-0,4$ & $-0,30$ & 4,69 & $-0,56$ \\
\hline & B & 24,4 & 24,4 & 24,4 & 24,4 & 24,1 & 24,7 & 24,5 & 24,43 & 24,5 & 24,7 & 24,8 & 24,67 & 24,5 & 24,7 & 24,8 & 24,67 & & \\
\hline & $\mathbf{L}$ & 96,1 & 95,9 & 96,1 & 96,03 & 96 & 95,9 & 96,2 & 96,03 & 93,2 & 93,2 & 93,2 & 93,20 & 93 & 93,4 & 93,2 & 93,2 & & \\
\hline 8 & A & -1 & $-1,1$ & $-1,1$ & $-1,06$ & $-1,1$ & $-1,1$ & $-1,1$ & $-1,1$ & -1 & -1 & $-1,1$ & $-1,03$ & $-1,1$ & $-1,1$ & $-1,1$ & $-1,10$ & 2,96 & $-0,02$ \\
\hline & B & 20,5 & 19,9 & 20 & 20,13 & 20 & 19,9 & 20 & 19,96 & 21,1 & 19 & 21 & 21,00 & 20,8 & 20,9 & 20,9 & 20,87 & & \\
\hline & $\mathbf{L}$ & 93,9 & 93,9 & 94,2 & 94 & 94,2 & 94,2 & 94,3 & 94,23 & 90,9 & 91,1 & 913 & 91,1 & 91,1 & 91,1 & 91,4 & 91,2 & & \\
\hline 9 & A & $\begin{array}{l}-1,6 \\
\end{array}$ & $-1,6$ & $-1,6$ & $-1,6$ & $-1,6$ & $\begin{array}{l}-1,6 \\
\end{array}$ & $\begin{array}{l}-1,5 \\
\end{array}$ & $\begin{array}{l}-1,56 \\
\end{array}$ & $-1,1$ & $-1,1$ & $-1,1$ & $-1,10$ & $\begin{array}{l}-1,2 \\
\end{array}$ & $\begin{array}{l}-1,2 \\
\end{array}$ & $-1,1$ & $-1,17$ & 3,30 & $-0,30$ \\
\hline & B & 17,3 & 17,4 & 17,3 & 17,33 & 17,5 & 17,8 & 17,8 & 17,7 & 18,6 & 19 & 18,9 & 18,83 & 18,7 & 18,8 & 18,8 & 18,77 & & \\
\hline & $\mathbf{L}$ & 97,1 & 95,4 & 95,7 & 96,7 & 95 & 95,5 & 95,5 & 95,33 & 92,5 & 89,9 & 90,6 & 91 & 90,3 & 90,7 & 90,6 & 90,53 & & \\
\hline 10 & A & 0,6 & 0,6 & 0,6 & 0,6 & $-0,6$ & $-0,6$ & $-0,6$ & $-0,6$ & 0,1 & 0,8 & 0,7 & 0,53 & 0,8 & 0,8 & 0,8 & 0,80 & 5,28 & 0,04 \\
\hline & B & 27,3 & 27,1 & 27,1 & 27,16 & 26,8 & 27 & 27,3 & 27,03 & 25,1 & 25,8 & 26,1 & 25,67 & 26,3 & 26,2 & 26,2 & 26,23 & & \\
\hline
\end{tabular}


Quadro 4- Leituras de cor das amostras de E-max Press na espessura de 1,0mm cimentados com cimento autoadesivo.

\begin{tabular}{|c|c|c|c|c|c|c|c|c|c|c|c|c|c|c|c|c|c|c|c|}
\hline \multicolumn{20}{|c|}{ E-max Press 1,0mm Rely-X U200 } \\
\hline \multirow{2}{*}{$\begin{array}{c}\text { Corpo de } \\
\text { Prova }\end{array}$} & \multirow{2}{*}{ EIXO } & \multicolumn{4}{|c|}{ LEITURA INICIA Branco } & \multicolumn{4}{|c|}{ LEITURA INICIAL Preto } & \multicolumn{4}{|c|}{ LEITURA Final Branco } & \multicolumn{4}{|c|}{ LEITURA FINAL Preto } & \multicolumn{2}{|c|}{$\Delta$} \\
\hline & & LI1 & LI2 & LI3 & MEDIA LI & LF1 & LF2 & LF3 & MEDIA LF & LI1 & LI2 & LI3 & MEDIA LI & LF1 & LF2 & LF3 & MEDIA LF & $\Delta \mathrm{E}$ & $\Delta T$ \\
\hline \multirow[t]{3}{*}{1} & $\mathbf{L}$ & 90,2 & 90,1 & 90,1 & 90,13 & 90,3 & 90,4 & 90,5 & 90,4 & 90,2 & 90,4 & 89,7 & 90,10 & 90 & 90,1 & 903 & 90,13 & \multirow{3}{*}{0,37} & \multirow{3}{*}{0,07} \\
\hline & $\mathbf{A}$ & $-0,7$ & $-0,7$ & $-0,7$ & $-0,7$ & $-0,7$ & $-0,7$ & $-0,7$ & $-0,7$ & $-0,6$ & $-0,5$ & $-0,5$ & $-0,53$ & $-0,6$ & $-0,5$ & $-0,5$ & $-0,53$ & & \\
\hline & B & 18,3 & 18,3 & 18,2 & 18,26 & 18,4 & 18,5 & 18,5 & 18,46 & 18,7 & 18,7 & 18,4 & 18,60 & 18,8 & 19,1 & 19,1 & 19,00 & & \\
\hline \multirow[t]{3}{*}{2} & $\mathbf{L}$ & 92,1 & 92 & 92 & 92,03 & 92 & 92,1 & 92,1 & 92,06 & 89,4 & 89,5 & 89,4 & 89,43 & 89,1 & 89,2 & 89,8 & 89,37 & \multirow{3}{*}{2,74} & \multirow{3}{*}{0,17} \\
\hline & $a$ & $-0,6$ & $-0,6$ & $-0,6$ & $-0,6$ & $-0,6$ & $-0,6$ & $-0,6$ & $-0,6$ & $-0,7$ & $-0,6$ & $-0,6$ & $-0,63$ & $-0,7$ & $-0,7$ & $-0,7$ & $-0,70$ & & \\
\hline & b & 19,8 & 19,9 & 19,8 & 19,83 & 19,8 & 19,9 & 19,9 & 19,86 & 18,9 & 19 & 19 & 18,97 & 19 & 19,1 & 19,4 & 19,17 & & \\
\hline \multirow[t]{3}{*}{3} & $\mathbf{L}$ & 90,8 & 90,6 & 90,6 & 90,66 & 90,8 & 90,7 & 90,9 & 90,8 & 90,7 & 90,7 & 90,3 & 90,57 & 90,8 & 90,4 & 90,8 & 90,67 & \multirow{3}{*}{0,74} & \multirow{3}{*}{$-0,04$} \\
\hline & $a$ & $-0,2$ & $-0,1$ & $-0,1$ & $-0,13$ & $-0,1$ & $-0,1$ & $-0,2$ & $-0,13$ & 0,2 & 0,2 & 0,3 & 0,23 & 0,3 & 0,3 & 0,2 & 0,27 & & \\
\hline & b & 21 & 20,9 & 21,1 & 21 & 21 & 20,9 & 20,9 & 20,93 & 21,8 & 21,8 & 21,3 & 21,63 & 21,7 & 21,4 & 21,7 & 21,60 & & \\
\hline \multirow[t]{3}{*}{4} & $\mathbf{L}$ & 92,1 & 92,2 & 91,9 & 92,06 & 92,1 & 92,2 & 92,1 & 92,13 & 91,9 & 91,7 & 91,9 & 91,83 & 92,3 & 92,3 & 92,6 & 92,40 & \multirow{3}{*}{0,68} & \multirow{3}{*}{0,66} \\
\hline & $a$ & $-0,8$ & $-0,8$ & $-0,8$ & $-0,8$ & $-0,8$ & $-0,8$ & $-0,8$ & $-0,8$ & $-0,5$ & $-0,5$ & $-0,5$ & $-0,50$ & $-0,5$ & $-0,5$ & $-0,5$ & $-0,50$ & & \\
\hline & b & 19,4 & 19,3 & 19,2 & 19,3 & 19,3 & 19,3 & 19,4 & 19,33 & 19,9 & 19,8 & 19,9 & 19,87 & 20,3 & 20,2 & 20,5 & 20,33 & & \\
\hline \multirow[t]{3}{*}{5} & $\mathbf{L}$ & 88,4 & 88,6 & 88,4 & 88,46 & 88,2 & 88,2 & 88,3 & 88,23 & 87,5 & 87,2 & 87,2 & 87,30 & 87,3 & 87,4 & 87,4 & 87,37 & \multirow{3}{*}{1,34} & \multirow{3}{*}{$-0,16$} \\
\hline & $a$ & $-0,5$ & $-0,5$ & $-0,5$ & $-0,5$ & $-0,6$ & $-0,6$ & $-0,6$ & $-0,6$ & $-0,3$ & $-0,2$ & $-0,2$ & $-0,23$ & $-0,2$ & $-0,2$ & $-0,2$ & $-0,20$ & & \\
\hline & b & 19,3 & 19,5 & 19,3 & 19,33 & 19,1 & 19,4 & 19,5 & 19,33 & 20,1 & 20 & 19,8 & 19,97 & 19,9 & 20 & 19,8 & 19,90 & & \\
\hline 6 & $\mathbf{L}$ & 93,4 & 94 & 93,5 & 93,63 & 93,5 & 93,4 & 93,5 & 93,46 & 93,6 & 93,4 & 93,7 & 93,57 & 93,6 & 93,9 & 93,9 & 93,80 & & \\
\hline & $a$ & $-0,7$ & $-0,7$ & $-0,7$ & $-0,7$ & $-0,7$ & $-0,7$ & $-0,7$ & $-0,7$ & $-0,4$ & $-0,3$ & -03 & $-0,33$ & $-0,3$ & $-0,3$ & $-0,3$ & $-0,30$ & 0,50 & 0,10 \\
\hline & b & 19,8 & 19,7 & 19,8 & 19,8 & 19,9 & 19,8 & 19,7 & 19,8 & 20,1 & 20 & 20,20 & 20,10 & 20,2 & 20,1 & 20,4 & 20,23 & & \\
\hline 7 & $\mathrm{~L}$ & 91,8 & 92,4 & 92 & 92,06 & 92,7 & 92,3 & 92,1 & 92,36 & 92 & 92,2 & 92,4 & 92,20 & 92,7 & 92,8 & 93,4 & 92,97 & & \\
\hline & $a$ & $-0,7$ & $-0,7$ & $-0,7$ & $-0,7$ & $-0,7$ & $-0,7$ & $-0,7$ & $-0,7$ & $-0,8$ & $-0,5$ & $-0,5$ & $-0,60$ & $-0,5$ & $-0,5$ & $-0,5$ & $-0,50$ & 0,85 & 0,63 \\
\hline & b & 17,9 & 18,2 & 18,2 & 18,1 & 18,4 & 18,2 & 18,1 & 18,23 & 18,8 & 18,9 & 19,1 & 18,93 & 19,3 & 19,5 & 19,7 & 19,50 & & \\
\hline 8 & $\mathbf{L}$ & 91,6 & 91,7 & 91,9 & 91,73 & 92 & 92,1 & 92 & 92,03 & 90,6 & 90,8 & 90,3 & 90,57 & 91,1 & 90,7 & 91,3 & 91,03 & & \\
\hline & $a$ & $-0,9$ & $-0,9$ & $-0,9$ & $-0,9$ & $-0,9$ & $-0,9$ & $-0,9$ & $-0,9$ & $-0,6$ & $-0,5$ & $-0,5$ & $-0,53$ & $-0,4$ & $-0,5$ & $-0,5$ & $-0,47$ & 1,32 & 0,28 \\
\hline & b & 17,7 & 17,6 & 17,9 & 17,73 & 17,9 & 17,8 & 17,8 & 17,83 & 17,2 & 17,2 & 17,3 & 17,23 & 17,5 & 17,5 & 17,8 & 17,60 & & \\
\hline 9 & L & 90,1 & 90,1 & 90,6 & 90,26 & 90,6 & 90,3 & 90,4 & 90,43 & 89,9 & 89,3 & 89,3 & 89,50 & 89,2 & 89,5 & 89,7 & 89,47 & & \\
\hline & $a$ & $-0,6$ & $-0,6$ & $-0,6$ & $-0,6$ & $-0,6$ & $-0,6$ & $-0,6$ & $-0,6$ & $-0,4$ & $-0,4$ & $-0,3$ & 0,37 & $-0,2$ & $-0,2$ & $-0,1$ & $-0,17$ & 0,86 & 0,33 \\
\hline & b & 19,4 & 19,2 & 19,2 & 19,26 & 19,3 & 19,4 & 19,5 & 19,4 & 19,7 & 19,4 & 19,6 & 19,57 & 19,7 & 20,1 & 20,4 & 20,07 & & \\
\hline & $\mathbf{L}$ & 89,9 & 89,9 & 89,9 & 89,9 & 90,1 & 90 & 90 & 90,03 & 87,7 & 87,6 & 87,9 & 87,73 & 87,4 & 87,8 & 87,7 & 87,63 & & \\
\hline 10 & $\mathbf{a}$ & $-0,1$ & 0 & $-0,1$ & 0,06 & 0 & $-0,1$ & $-0,1$ & $-0,06$ & 0,3 & 0,2 & 0,2 & 0,23 & 0,4 & 0,4 & 0,4 & 0,40 & 2,20 & 0,39 \\
\hline & b & 20,7 & 20,6 & 20,4 & 20,56 & 20,8 & 20,7 & 20,7 & 20,73 & 20,3 & 20,5 & 20,1 & 20,30 & 20,6 & 21,1 & 20,9 & 20,87 & & \\
\hline
\end{tabular}


Quadro 5- Leituras de cor das amostras de ZirPress na espessura de 0,5mm cimentados com cimento fotoativado.

\begin{tabular}{|c|c|c|c|c|c|c|c|c|c|c|c|c|c|c|c|c|c|c|c|}
\hline \multicolumn{20}{|c|}{ ZirPress $0,5 \mathrm{~mm}$ Variolink II } \\
\hline \multirow{2}{*}{$\begin{array}{l}\text { Corpo de } \\
\text { Prova }\end{array}$} & \multirow{2}{*}{ EIXO } & \multicolumn{4}{|c|}{ LEITURA INICIA Branco } & \multicolumn{4}{|c|}{ LEITURA INICIAL Preto } & \multicolumn{4}{|c|}{ LEITURA Final Branco } & \multicolumn{4}{|c|}{ LEITURA FINAL Preto } & \multicolumn{2}{|c|}{$\Delta$} \\
\hline & & LI1 & $\mathrm{LI} 2$ & LI3 & MEDIA LI & LF1 & LF2 & LF3 & MEDIA LF & LI1 & $\mathrm{L} 12$ & $\mathrm{LI3}$ & MEDIA LI & LF1 & LF2 & LF3 & MEDIA LF & $\Delta \mathrm{E}$ & $\Delta \mathrm{T}$ \\
\hline \multirow[t]{3}{*}{1} & $\mathbf{L}$ & 91,3 & 91,3 & 91,3 & 91,3 & 91,4 & 91,2 & 91,4 & 91,33 & 88,5 & 88,7 & 88,8 & 88,67 & 88,6 & 88,8 & 88,7 & 88,70 & \multirow{3}{*}{2,64} & \multirow{3}{*}{0,07} \\
\hline & $\mathbf{a}$ & $-1,6$ & $-1,6$ & $-1,6$ & $-1,6$ & $-1,6$ & $-1,6$ & $-1,6$ & $-1,6$ & $-1,4$ & $-1,3$ & $-1,4$ & $-1,37$ & $-1,4$ & $-1,4$ & $-1,4$ & $-1,40$ & & \\
\hline & b & 21,2 & 21 & 21,3 & 21,16 & 20,8 & 21,2 & 21,2 & 21,06 & 20,7 & 21,3 & 21,6 & 21,20 & 21,1 & 21 & 21 & 21,03 & & \\
\hline \multirow[t]{3}{*}{2} & $\mathbf{L}$ & 90,7 & 90,8 & 90,5 & 90,66 & 90,7 & 90,6 & 90,7 & 90,66 & 88,7 & 88,6 & 88,6 & 88,63 & 88,6 & 88,6 & 88,4 & 88,53 & \multirow{3}{*}{2,38} & \multirow{3}{*}{$-0,20$} \\
\hline & $a$ & $-0,2$ & $\begin{array}{l}-0,3 \\
\end{array}$ & $-0,1$ & $-0,2$ & $-0,1$ & $-0,2$ & $-0,1$ & $-0,13$ & 0,1 & 0,1 & 0,1 & 0,10 & 0,1 & 0,1 & 0,1 & 0,10 & & \\
\hline & b & 25,8 & 25,5 & 26,3 & 25,86 & 26,4 & 26 & 26,1 & 26,16 & 27,2 & 27 & 27,0 & 27,07 & 27,1 & 27,1 & 27,1 & 27,10 & & \\
\hline \multirow[t]{3}{*}{3} & $\mathbf{L}$ & 93,6 & 93,6 & 93,6 & 93,6 & 93,6 & 93,8 & 93,6 & 93,66 & 89,9 & 90,2 & 90,2 & 90,10 & 90,4 & 90,6 & 90,8 & 90,6 & \multirow{3}{*}{3,91} & \multirow{3}{*}{0,20} \\
\hline & $a$ & $-1,6$ & $-1,6$ & $-1,6$ & $-1,6$ & $-1,6$ & $-1,7$ & $-1,6$ & $-1,63$ & -1 & -1 & -1 & -1 & -1 & $-1,1$ & $-1,1$ & $-1,07$ & & \\
\hline & b & 19,8 & 19,7 & 19,8 & 19,76 & 19,6 & 19,2 & 19,4 & 19,4 & 21,6 & 21,3 & 21,3 & 21,40 & 21,6 & 21,8 & 21,6 & 21,67 & & \\
\hline \multirow[t]{3}{*}{4} & $\mathbf{L}$ & 91,7 & 91,8 & 92,3 & 91,93 & 91,8 & 91,8 & 92,2 & 91,93 & 90,2 & 90,3 & 90,6 & 90,37 & 90,6 & 90,6 & 90,7 & 90,63 & \multirow{3}{*}{2,16} & \multirow{3}{*}{0,26} \\
\hline & $a$ & $-1,1$ & $-1,1$ & $-1,1$ & $-1,1$ & -1 & -1 & $-1,1$ & $-1,03$ & $-0,3$ & $-0,3$ & $-0,4$ & $-0,33$ & $-0,4$ & $-0,3$ & $-0,4$ & $-0,37$ & & \\
\hline & b & 226 & 22,5 & 22,3 & 22,46 & 22,5 & 22,6 & 22,6 & 22,56 & 23,7 & 23,8 & 23,7 & 23,73 & 23,5 & 23,4 & 23,5 & 23,47 & & \\
\hline \multirow[t]{3}{*}{5} & L & 92,3 & 92,3 & 92,3 & 92,3 & 92,4 & 92,5 & 92,4 & 92,43 & 91,1 & 91 & 91,2 & 91,10 & 90,9 & 91 & 91 & 90,97 & \multirow{3}{*}{1,55} & \multirow{3}{*}{$-0,08$} \\
\hline & $a$ & $-1,4$ & $-1,4$ & $-1,4$ & $-1,4$ & $-1,5$ & $-1,4$ & $-1,5$ & $-1,46$ & -1 & $-0,9$ & $-0,9$ & $-0,93$ & -1 & -1 & $-0,8$ & $-0,93$ & & \\
\hline & b & 22,1 & 21,8 & 21,9 & 21,93 & 22 & 22,2 & 21 & 21,73 & 22,9 & 22,5 & 23 & 22,80 & 22,7 & 22,6 & 22,8 & 22,7 & & \\
\hline 6 & $\mathbf{L}$ & 94,5 & 94,4 & 94,6 & 94,5 & 94,6 & 94,6 & 94,7 & 9463 , & 92,4 & 92 & 91,9 & 92,1 & 92 & 92,1 & 92 & 92,03 & & \\
\hline & $\mathbf{a}$ & 0,1 & 0,1 & 0 & 0,06 & 0,1 & 0,1 & 0,1 & 0,1 & 0,5 & 0,4 & 0,4 & 0,43 & 0,4 & 0,3 & 0,4 & 0,37 & 2,43 & $-0,05$ \\
\hline & b & 27,5 & 27,3 & 26,7 & 27,16 & 27,5 & 27,3 & 27,4 & 27,4 & 27,8 & 26,7 & 26,6 & 27,03 & 27,1 & 26,6 & 26,8 & 26,83 & & \\
\hline 7 & $\mathbf{L}$ & 93,4 & 93,4 & 93,6 & 93,46 & 93,5 & 93,4 & 93,4 & 93,43 & 93 & 92,2 & 91,9 & 92,37 & 92,4 & 92,1 & 92,2 & 92,23 & & \\
\hline & $\mathbf{a}$ & $-1,2$ & $-1,2$ & $-1,1$ & $-1,16$ & $-1,2$ & $-1,2$ & $-1,2$ & $-1,2$ & -1 & $-0,9$ & $-0,9$ & $-0,93$ & -1 & $-0,9$ & $-0,9$ & $-0,93$ & 1,19 & 0,31 \\
\hline & b & 21 & 20,9 & 21,7 & 21,2 & 21,7 & 21,5 & 21,1 & 21,43 & 19,9 & 20,8 & 21,7 & 20,8 & 20,7 & 21,5 & 21,8 & 21,33 & & \\
\hline 8 & $\mathbf{L}$ & 92,8 & 93,2 & 93,2 & 93,06 & 93,2 & 92,6 & 92,6 & 92,8 & 90 & 90,1 & 89,9 & 90 & 89,7 & 89,6 & 90 & 89,77 & & \\
\hline & $\mathbf{a}$ & $-0,9$ & -1 & -1 & $-0,96$ & $-0,9$ & -1 & -1 & $-0,96$ & $-0,6$ & $-0,6$ & $-0,6$ & $-0,60$ & $-0,6$ & $-0,6$ & $-0,6$ & $-0,6$ & 3,13 & $-0,25$ \\
\hline & b & 23,9 & 23,9 & 24 & 23,93 & 24,5 & 24,2 & 24,3 & 24,33 & 24,4 & 24,6 & 24,3 & 24,43 & 24,4 & 24,4 & 24,5 & 24,43 & & \\
\hline 9 & $\mathbf{L}$ & 88,7 & 88,8 & 89,3 & 88,93 & 89,4 & 88,7 & 88,7 & 88,93 & 86,8 & 86,4 & 86,7 & 86,63 & 86,6 & 87 & 87 & 86,87 & & \\
\hline & $a$ & 0,4 & 0,3 & 0,3 & 0,33 & 0,3 & 0,4 & 0,4 & 0,36 & 0,4 & 0,4 & 0,4 & 0,40 & 0,5 & 0,5 & 0,5 & 0,5 & 2,38 & $-0,05$ \\
\hline & b & 25,7 & 25,4 & 25,5 & 25,53 & 25,8 & 25,9 & 25,8 & 25,83 & 26,2 & 26 & 26,2 & 26,13 & 26 & 26,2 & 26,3 & 26,17 & & \\
\hline & $L$ & 91,5 & 91,1 & 92 & 91,53 & 91,1 & 91,3 & 91,2 & 91,2 & 86,8 & 87,3 & 87,5 & 87,2 & 87,8 & 87,6 & 87,8 & 87,73 & & \\
\hline 10 & $a$ & 0 & 0 & 0 & 0 & 0 & 0 & 0 & 0 & 0,10 & 0,10 & 0,10 & 0,10 & 0 & 0,1 & 0,3 & 0,13 & 4,39 & 0,21 \\
\hline & b & 25,8 & 26,4 & 25,7 & 25,96 & 26,1 & 25,7 & 25,9 & 25,9 & 25,4 & 25,3 & 25,1 & 25,27 & 24,7 & 25,8 & 25,7 & 25,40 & & \\
\hline
\end{tabular}


Quadro 6- Leituras de cor das amostras de ZirPress na espessura de 1,0mm cimentados com cimento fotoativado.

\begin{tabular}{|c|c|c|c|c|c|c|c|c|c|c|c|c|c|c|c|c|c|c|c|}
\hline \multicolumn{20}{|c|}{ ZirPress 1,0mm Variolink II } \\
\hline \multirow{2}{*}{$\begin{array}{c}\text { Corpo- } \\
\text { de-Prova }\end{array}$} & \multirow{2}{*}{ EIXO } & \multicolumn{4}{|c|}{ LEITURA INICIA Branco } & \multicolumn{4}{|c|}{ LEITURA INICIAL Preto } & \multicolumn{4}{|c|}{ LEITURA Final Branco } & \multicolumn{4}{|c|}{ LEITURA FINAL Preto } & \multicolumn{2}{|c|}{$\Delta$} \\
\hline & & LI1 & LI2 & LI3 & MEDIA LI & LF1 & LF2 & LF3 & MEDIA LF & Ll1 & LI2 & LI3 & MEDIA LI & LF1 & LF2 & LF3 & MEDIA LF & $\Delta \mathrm{E}$ & $\Delta \mathbf{T}$ \\
\hline \multirow[t]{3}{*}{1} & $\mathbf{L}$ & 94,1 & 94,3 & 94,1 & 94,16 & 95,7 & 95 & 95,2 & 95,3 & 87,7 & 87,6 & 88,1 & 87,80 & 87,7 & 88,1 & 88,2 & 88,00 & \multirow{3}{*}{6,73} & \multirow{3}{*}{$-1,12$} \\
\hline & $\mathbf{a}$ & 0,2 & 0,2 & 0,1 & 0,16 & 0,4 & 0,4 & 0,4 & 0,4 & 2,1 & 2,2 & 2,1 & 2,13 & 2,4 & 2,3 & 2,3 & 2,33 & & \\
\hline & b & 25,9 & 26,3 & 25,9 & 26,03 & 27 & 26,8 & 26,9 & 26,9 & 25,1 & 25,1 & 25,1 & 25,10 & 25,4 & 25,4 & 25 & 25,27 & & \\
\hline \multirow[t]{3}{*}{2} & $\mathbf{L}$ & 89,1 & 89,4 & 89,26 & 89,26 & 89,4 & 89,4 & 89,3 & 89,36 & 86,6 & 86,9 & 86,3 & 86,60 & 86,3 & 86,4 & 86,6 & 86,43 & \multirow{3}{*}{3,16} & \multirow{3}{*}{0,04} \\
\hline & $\mathbf{a}$ & 0,3 & 0,3 & 0,3 & 0,3 & 0,2 & 0,2 & 0,2 & 0,2 & 0,3 & 0,2 & 0,1 & 0,20 & 0,2 & 0,2 & 0,2 & 0,20 & & \\
\hline & b & 24,8 & 25,1 & 25,2 & 25,03 & 25,2 & 25,2 & 25,3 & 25,23 & 23,4 & 23,4 & 23,2 & 23,33 & 23,1 & 23,1 & 23,1 & 23,10 & & \\
\hline \multirow[t]{3}{*}{3} & $L$ & 84,6 & 84,8 & 85 & 84,8 & 84,9 & 84,9 & 85,4 & 85,06 & 83,5 & 83,2 & 83,8 & 83,50 & 82,8 & 82,7 & 82,9 & 82,80 & \multirow{3}{*}{1,33} & \multirow{3}{*}{0,10} \\
\hline & $a$ & 0,6 & 0,5 & 0,5 & 0,53 & 0,5 & 0,4 & 0,4 & 0,43 & 0,3 & 0,4 & 0,4 & 0,37 & 0,3 & 0,3 & 0,4 & 0,33 & & \\
\hline & b & 22,1 & 22,1 & 22 & 22,03 & 22,6 & 22,5 & 22,7 & 22,6 & 21,6 & 22 & 22 & 21,87 & 21,7 & 21,8 & 21,9 & 21,80 & & \\
\hline \multirow[t]{3}{*}{4} & $\mathbf{L}$ & 91,9 & 91,9 & 92,1 & 91,96 & 92,6 & 92,4 & 92,5 & 92,5 & 88,4 & 88,3 & 88,6 & 88,43 & 88,9 & 88,8 & 88,9 & 88,87 & \multirow{3}{*}{4,26} & \multirow{3}{*}{$-0,61$} \\
\hline & $a$ & 0,4 & 0,3 & 0,3 & 0,33 & 0,6 & 0,5 & 0,6 & 0,56 & 0,1 & 0,1 & 0 & 0,07 & 0 & 0 & 0 & 0 & & \\
\hline & b & 26 & 25,7 & 25,8 & 25,83 & 26,8 & 26,6 & 26,8 & 26,73 & 23,4 & 23,5 & 23,5 & 23,47 & 23,9 & 23,5 & 23,4 & 23,60 & & \\
\hline \multirow[t]{3}{*}{5} & $\mathbf{L}$ & 88,4 & 88,6 & 88,5 & 88,5 & 89 & 88,9 & 89,3 & 93,7 & 87 & 86,7 & 86,9 & 86,87 & 86,6 & 86,8 & 88,9 & 86,70 & \multirow{3}{*}{1,76} & \multirow{3}{*}{$-0,53$} \\
\hline & $a$ & 0,1 & 0,1 & 0,2 & 0,13 & 0,3 & 0,2 & 0,3 & 0,26 & 0,5 & 0,4 & 0,5 & 0,47 & 0,5 & 0,4 & 0,4 & 0,43 & & \\
\hline & b & 24,2 & 24 & 24,2 & 24,2 & 24,7 & 24,4 & 25 & 24,7 & 23,8 & 23,1 & 24 & 23,63 & 23,7 & 23,4 & 23,3 & 23,47 & & \\
\hline 6 & $\mathbf{L}$ & 89,8 & 89,9 & 90 & 89,9 & 90,7 & 90,8 & 90,6 & 90,7 & 90,7 & 90,2 & 89,9 & 90,27 & 90,5 & 90,4 & 90,6 & 90,5 & & \\
\hline & a & $-0,1$ & $-0,1$ & $-0,1$ & $-0,1$ & $-0,2$ & $-0,2$ & $-0,2$ & $-0,2$ & 0,6 & 0,6 & 0,7 & 0,63 & 0,6 & 0,7 & 0,6 & 0,63 & 4,05 & $-0,74$ \\
\hline & b & 21,1 & 21,2 & 21,2 & 21,16 & 21,8 & 21,8 & 21,8 & 21,8 & 25,4 & 24,9 & 25,1 & 25,13 & 25,1 & 25,6 & 25,2 & 25,30 & & \\
\hline 7 & $\mathbf{L}$ & 91,5 & 91,7 & 91,7 & 91,63 & 92,2 & 92,3 & 92,3 & 92,26 & 90,1 & 89,8 & 89,8 & 89,90 & 90,2 & 90 & 90,2 & 90,13 & & \\
\hline & $\mathbf{a}$ & $-1,2$ & $-1,2$ & $-1,1$ & $-1,16$ & $-1,1$ & $-1,1$ & $-1,1$ & $-1,1$ & $-0,7$ & $-0,7$ & $-0,7$ & $-0,70$ & $-0,5$ & $-0,7$ & $-0,6$ & $-0,6$ & 1,80 & $-0,39$ \\
\hline & b & 21,9 & 21,8 & 22 & 21,9 & 21,9 & 22,2 & 22,2 & 22,1 & 22 & 21,8 & 21,9 & 21,90 & 21,9 & 22,1 & 22 & 22 & & \\
\hline 8 & L & 87,1 & 87,5 & 87,8 & 87,46 & 88,1 & 88,1 & 88,2 & 88,13 & 83,4 & 83,5 & 83,4 & 83,43 & 83,1 & 83,5 & 83,5 & 83,37 & & \\
\hline & $\mathbf{a}$ & $-1,1$ & $-0,9$ & -1 & $-0,9$ & $-0,9$ & $-0,9$ & $-0,9$ & $-0,9$ & $-0,7$ & $-0,8$ & $-0,8$ & $-0,77$ & $-0,9$ & $-0,9$ & $-0,9$ & $-0,90$ & 4,15 & $-0,81$ \\
\hline & b & 19,6 & 19,9 & 19,9 & 19,8 & 20,5 & 20,4 & 20,6 & 20,5 & 18,6 & 19 & 18,9 & 18,83 & 18,8 & 18,9 & 19 & 18,90 & & \\
\hline 9 & $\mathbf{L}$ & 92,7 & 93,4 & 92,8 & 92,96 & 92,7 & 93,1 & 93,1 & 92,96 & 89,7 & 89,9 & 89,7 & 89,77 & 89,9 & 90,2 & 90,4 & 90,17 & & \\
\hline & $\mathbf{a}$ & $-0,6$ & $-0,7$ & $-0,6$ & $-0,63$ & $-0,5$ & $-0,5$ & $-0,5$ & $-0,5$ & $-0,6$ & $-0,7$ & $-0,7$ & $-0,67$ & $-0,7$ & $-0,7$ & $-0,6$ & $-0,67$ & 3,27 & $-0,21$ \\
\hline & b & 22,9 & 22,5 & 22,5 & 22,63 & 23,1 & 23,1 & 23,5 & 23,23 & 21,9 & 22 & 22 & 21,97 & 21,7 & 22 & 22,2 & 21,97 & & \\
\hline & $\mathbf{L}$ & 89,8 & 90 & 90 & 89,93 & 89,8 & 89,9 & 89,8 & 89,83 & 88,2 & 87,9 & 88 & 88,03 & 88,3 & 88,3 & 88 & 88,20 & & \\
\hline 10 & $a$ & $-0,6$ & $-0,7$ & $-0,7$ & $-0,66$ & $-0,7$ & $-0,7$ & $-0,6$ & $\begin{array}{l}-0,66 \\
\end{array}$ & $\begin{array}{l}-0,3 \\
\end{array}$ & $\begin{array}{l}-0,3 \\
\end{array}$ & $-0,2$ & $-0,27$ & $-0,3$ & $-0,3$ & $-0,3$ & $-0,30$ & 1,96 & $-0,17$ \\
\hline & b & 21,8 & 22 & 22,1 & 21,96 & 22,3 & 22,2 & 22,4 & 22,3 & 21,8 & 21,8 & 21,6 & 21,73 & 21,5 & 21,8 & 21,7 & 21,67 & & \\
\hline
\end{tabular}


Quadro 7- Leituras de cor das amostras de ZirPress na espessura de 0,5mm cimentados com cimento autoadesivo.

\begin{tabular}{|c|c|c|c|c|c|c|c|c|c|c|c|c|c|c|c|c|c|c|c|}
\hline \multicolumn{20}{|c|}{ ZirPress 0,5mm Rely-X U200 } \\
\hline \multirow{2}{*}{$\begin{array}{c}\text { Corpo de } \\
\text { Prova }\end{array}$} & \multirow{2}{*}{ EIXO } & \multicolumn{4}{|c|}{ LEITURA INICIA Branco } & \multicolumn{4}{|c|}{ LEITURA INICIAL Preto } & \multicolumn{4}{|c|}{ LEITURA Final Branco } & \multicolumn{4}{|c|}{ LEITURA FINAL Preto } & \multicolumn{2}{|c|}{$\Delta$} \\
\hline & & LI1 & $\mathrm{LI} 2$ & LI3 & MEDIA LI & LF1 & LF2 & LF3 & MEDIA LF & LI1 & LI2 & LI3 & MEDIA LI & LF1 & LF2 & LF3 & MEDIA LF & $\Delta \mathrm{E}$ & $\Delta T$ \\
\hline \multirow[t]{3}{*}{1} & $\mathbf{L}$ & 90,3 & 90,3 & 90,3 & 90,3 & 90,5 & 90,5 & 90,4 & 90,46 & 86,5 & 86,6 & 86,3 & 86,47 & 86 & 86,3 & 86,5 & 86,27 & \multirow{3}{*}{4,54} & \multirow{3}{*}{$-0,12$} \\
\hline & $\mathbf{a}$ & $-0,6$ & $-0,6$ & $-0,7$ & $-0,63$ & $-0,7$ & $-0,6$ & $-0,7$ & $-0,66$ & $-0,4$ & $-0,4$ & $-0,4$ & $-0,40$ & $-0,2$ & $-0,2$ & $-0,3$ & $-0,23$ & & \\
\hline & b & 23,9 & 23,8 & 23,6 & 23,76 & 23,5 & 23,3 & 23,4 & 23,4 & 25,7 & 26,8 & 25,9 & 26,13 & 25,8 & 26,3 & 26,1 & 26,07 & & \\
\hline \multirow[t]{3}{*}{2} & $\mathbf{L}$ & 95,8 & 95,9 & 95,7 & 95,8 & 95,8 & 95,8 & 95,8 & 95,8 & 92 & 92 & 92 & 92,00 & 92,2 & 92 & 92,3 & 92,17 & \multirow{3}{*}{4,81} & \multirow{3}{*}{$-0,15$} \\
\hline & $\mathbf{a}$ & -1 & -1 & -1 & -1 & $-1,1$ & $-1,1$ & $-1,1$ & $-1,1$ & $-0,5$ & $-0,4$ & $-0,4$ & $-0,43$ & $-0,4$ & $-0,5$ & $-0,5$ & $-0,47$ & & \\
\hline & b & 24,2 & 24,3 & 24 & 24,16 & 23,9 & 23,4 & 24 & 23,76 & 27,3 & 27,2 & 26,7 & 27,07 & 26,9 & 27,2 & 26,5 & 26,87 & & \\
\hline \multirow[t]{3}{*}{3} & $\mathbf{L}$ & 93,7 & 93,5 & 93,3 & 93,5 & 93,4 & 93,6 & 93,5 & 93,5 & 87,4 & 84,8 & 84,6 & 85,6 & 85 & 84 & 84,5 & 84,50 & \multirow{3}{*}{7,91} & \multirow{3}{*}{0,84} \\
\hline & $\mathbf{a}$ & $\begin{array}{l}-0,3 \\
\end{array}$ & $\begin{array}{l}-0,3 \\
\end{array}$ & $\begin{array}{l}-0,3 \\
\end{array}$ & $\begin{array}{l}-0,3 \\
\end{array}$ & $\begin{array}{l}-0,2 \\
\end{array}$ & $\begin{array}{l}-0,2 \\
\end{array}$ & $\begin{array}{l}-0,2 \\
\end{array}$ & $-0,2$ & $-0,1$ & $-0,1$ & $-0,2$ & $-0,13$ & $-0,1$ & 0 & 0 & $\begin{array}{l}-0,03 \\
\end{array}$ & & \\
\hline & b & 22,9 & 22,9 & 22,7 & 22,83 & 23 & 23,2 & 23,2 & 23,13 & 23 & 23,3 & 23,2 & 23,17 & 23,5 & 23,4 & 23,6 & 23,50 & & \\
\hline \multirow[t]{3}{*}{4} & L & 96,8 & 97,1 & 97,2 & 97,03 & 97,1 & 97,6 & 97,2 & 97,3 & 90,6 & 90,2 & 90,3 & 90,37 & 90 & 90,4 & 90,2 & 90,20 & \multirow{3}{*}{6,93} & \multirow{3}{*}{$-0,08$} \\
\hline & $a$ & $-0,4$ & $-0,2$ & $-0,2$ & $-0,26$ & $\begin{array}{l}-0,3 \\
\end{array}$ & $\begin{array}{l}-0,3 \\
\end{array}$ & $-0,2$ & $-0,26$ & 0,2 & $-0,1$ & 0 & 0,03 & $-0,1$ & $-0,1$ & 0 & $\begin{array}{l}-0,03 \\
\end{array}$ & & \\
\hline & b & 26,5 & 26,2 & 26,2 & 26,3 & 26,7 & 26,4 & 26,4 & 26,5 & 28,5 & 27,8 & 28,2 & 28,17 & 27,7 & 27,9 & 28,4 & 28,00 & & \\
\hline \multirow[t]{3}{*}{5} & $\mathbf{L}$ & 92,9 & 93,1 & 93,5 & 93,16 & 93 & 93,2 & 93,1 & 93,1 & 87,1 & 87,3 & 87,4 & 87,27 & 87,3 & 86,4 & 87,3 & 87,00 & \multirow{3}{*}{6,30} & \multirow{3}{*}{0,22} \\
\hline & $a$ & 0,4 & 0,4 & 0,4 & 0,4 & 0,4 & 0,4 & 0,4 & 0,4 & 0,5 & 0,5 & 0,4 & 0,47 & 0,4 & 0,6 & 0,4 & 0,47 & & \\
\hline & b & 28,2 & 28,2 & 28,4 & 28,26 & 28,5 & 28,5 & 28,3 & 28,43 & 30,9 & 30,3 & 30,2 & 30,47 & 30,9 & 30,9 & 30,5 & 30,77 & & \\
\hline 6 & L & 91,5 & 91,6 & 91,7 & 91,6 & 91,7 & 91,7 & 91,8 & 91,73 & 87 & 86,8 & 86,6 & 86,8 & 86,9 & 87,1 & 87,1 & 87,03 & & \\
\hline & $\mathbf{a}$ & $-1,3$ & $-1,3$ & $-1,3$ & $-1,3$ & $-1,3$ & $-1,3$ & $-1,3$ & $-1,3$ & $-0,9$ & $-0,9$ & $-0,9$ & $-0,90$ & -1 & -1 & -1 & $-1,00$ & 5,23 & 0,10 \\
\hline & b & 20,5 & 20,4 & 20,1 & 20,33 & 20,4 & 20,6 & 20,7 & 20,56 & 22,6 & 22,3 & 22,2 & 22,37 & 22,7 & 22,8 & 22,4 & 22,63 & & \\
\hline 7 & $\mathbf{L}$ & 92,9 & 93,1 & 92,6 & 92,96 & 92,9 & 92,9 & 92,9 & 92,9 & 89,6 & 89,6 & 89,3 & 89,50 & 89,3 & 89,5 & 89,3 & 89,37 & & \\
\hline & $\mathbf{a}$ & 0,2 & 0,1 & 0,2 & 0,16 & 0,2 & 0,2 & 0,2 & 0,2 & 0,5 & 0,5 & 0,5 & 0,50 & 0,5 & 0,5 & 0,6 & 0,53 & 4,47 & 0,07 \\
\hline & b & 27,4 & 27,2 & 27,4 & 27,33 & 27,3 & 27,4 & 27,6 & 27,43 & 30,5 & 29,6 & 30,3 & 30,13 & 30 & 29,9 & 30,1 & 30,00 & & \\
\hline 8 & $\mathbf{L}$ & 96,2 & 96,3 & 96,1 & 96,2 & 96 & 96 & 96,2 & 96,06 & 88,4 & 87,9 & 88,1 & 88,13 & 88,1 & 88,2 & 87,6 & 87,97 & & \\
\hline & $\mathbf{a}$ & $-0,7$ & $-0,6$ & $-0,7$ & $-0,66$ & $-0,7$ & $-0,7$ & $-0,6$ & $-0,66$ & $-0,5$ & $-0,4$ & $-0,4$ & $-0,43$ & $-0,5$ & $-0,4$ & $-0,4$ & $-0,43$ & 8,09 & 0,27 \\
\hline & b & 25,7 & 26,5 & 25,9 & 26,03 & 25,9 & 26 & 26,3 & 26,06 & 26,4 & 26,7 & 26,6 & 26,57 & 26,1 & 26,5 & 26 & 26,20 & & \\
\hline 9 & $\mathbf{L}$ & 94,8 & 94,9 & 94,9 & 94,86 & 94,9 & 95,1 & 94,9 & 94,96 & 90,5 & 90,2 & 90,3 & 90,33 & 90,2 & 89,8 & 90,2 & 90,07 & & \\
\hline & $\mathbf{a}$ & $-1,2$ & $-1,1$ & $\begin{array}{l}-1,2 \\
\end{array}$ & $-1,16$ & $-1,1$ & $-1,1$ & $-1,1$ & $-1,13$ & $-0,7$ & $-0,8$ & $-0,8$ & $-0,77$ & $-0,8$ & $-0,7$ & $-0,7$ & $-0,73$ & 6,89 & 0,17 \\
\hline & b & 23,4 & 24,1 & 23,4 & 23,63 & 23,8 & 23,5 & 23,9 & 23,73 & 28,9 & 28,8 & 28,7 & 28,80 & 28,9 & 29,1 & 28,9 & 28,97 & & \\
\hline & $\mathbf{L}$ & 96,2 & 96,2 & 96,2 & 96,2 & 96,3 & 96,4 & 96,3 & 96,33 & 87,1 & 86,6 & 86,3 & 86,67 & 86,7 & 87,2 & 86,8 & 86,90 & & \\
\hline & $\mathbf{a}$ & 0,6 & 0,6 & 0,7 & 0,63 & 0,7 & 0,6 & 0,7 & 0,66 & 0,2 & 0,2 & 0,2 & 0,20 & 0,2 & 0,4 & 0,3 & 0,30 & 9,55 & $-0,13$ \\
\hline 10 & b & 26,4 & 26,6 & 27,1 & 26,7 & 27,1 & 27,3 & 27,3 & 27,23 & 26,7 & 26,5 & 26,3 & 26,50 & 26,5 & 27,3 & 26,7 & 26,83 & & \\
\hline
\end{tabular}


Quadro 8- Leituras de cor das amostras de ZirPress na espessura de 1,0mm cimentados com cimento autoadesivo.

\begin{tabular}{|c|c|c|c|c|c|c|c|c|c|c|c|c|c|c|c|c|c|c|c|}
\hline \multicolumn{20}{|c|}{ ZirPress 1,0mm Rely-X U200 } \\
\hline \multirow{2}{*}{$\begin{array}{c}\text { Corpo de } \\
\text { Prova }\end{array}$} & \multirow{2}{*}{ EIXO } & \multicolumn{4}{|c|}{ LEITURA INICIA Branco } & \multicolumn{4}{|c|}{ LEITURA INICIAL Preto } & \multicolumn{4}{|c|}{ LEITURA Final Branco } & \multicolumn{4}{|c|}{ LEITURA FINAL Preto } & \multicolumn{2}{|c|}{$\Delta$} \\
\hline & & LI1 & LI2 & LI3 & MEDIA LI & LF1 & LF2 & LF3 & MEDIA LF & LI1 & LI2 & LI3 & MEDIA LI & LF1 & LF2 & LF3 & MEDIA LF & $\Delta \mathrm{E}$ & $\Delta T$ \\
\hline \multirow[t]{3}{*}{1} & $\mathbf{L}$ & LI1 & LI2 & LI3 & MEDIA LI & LF1 & LF2 & LF3 & MEDIA LF & 80,7 & 81,1 & 81 & 80,93 & 80,7 & 81,2 & 81,1 & 81,00 & \multirow{3}{*}{6,86} & \multirow{3}{*}{$-0,26$} \\
\hline & $\mathbf{a}$ & 87,8 & 87,6 & 87,7 & 87,7 & 87,9 & 88,1 & 87,9 & 87,96 & $-0,4$ & $-0,4$ & $-0,4$ & $-0,40$ & $-0,4$ & $-0,4$ & $-0,4$ & $-0,40$ & & \\
\hline & b & $-0,1$ & $-0,2$ & $-0,2$ & $-0,16$ & $-0,2$ & $-0,2$ & $-0,2$ & $-0,2$ & 21,9 & 22,2 & 22 & 22,03 & 22,2 & 22,1 & 22,0 & 22,10 & & \\
\hline \multirow[t]{3}{*}{2} & $\mathbf{L}$ & 23,2 & 23,1 & 23,1 & 23,13 & 23 & 22,9 & 22,8 & 22,9 & 86,7 & 86,9 & 86,9 & 86,83 & 87 & 87 & 87,5 & 87,17 & \multirow{3}{*}{2,99} & \multirow{3}{*}{0} \\
\hline & $\mathbf{a}$ & 89,9 & 89,7 & 89,8 & 89,8 & 90 & 90,1 & 90,3 & 90,13 & 2,5 & 2,5 & 2,6 & 2,53 & 2,7 & 2,6 & 2,7 & 2,67 & & \\
\hline & b & 2,5 & 2,5 & 2,6 & 2,53 & 2,7 & 2,7 & 2,6 & 2,66 & 29,5 & 29,4 & 29,8 & 29,57 & 29,8 & 29,8 & 30,1 & 29,90 & & \\
\hline \multirow[t]{3}{*}{3} & $\mathbf{L}$ & 29,2 & 29,1 & 29,2 & 29,16 & 29,4 & 29,4 & 29,7 & 29,5 & 84,8 & 84,5 & 85,1 & 84,8 & 85,1 & 84,6 & 84,8 & 84,83 & \multirow{3}{*}{2,54} & \multirow{3}{*}{$-0,08$} \\
\hline & $\mathbf{a}$ & 87,4 & 87,1 & 87,4 & 87,3 & 87,2 & 87,1 & 87,1 & 87,13 & $-0,7$ & $-0,7$ & $-0,7$ & $-0,70$ & $-0,8$ & $-0,7$ & $-0,7$ & $-0,73$ & & \\
\hline & b & $-0,6$ & $-0,6$ & $-0,7$ & $-0,63$ & $-0,7$ & $-0,7$ & $-0,7$ & $-0,7$ & 24 & 24 & 23,8 & 23,93 & 24,2 & 24 & 24 & 24,07 & & \\
\hline \multirow[t]{3}{*}{4} & $\mathbf{L}$ & 23,6 & 23,3 & 23,5 & 23,46 & 23,3 & 23,4 & 23,3 & 23,33 & 84,9 & 84,8 & 84,6 & 84,77 & 84,8 & 84,9 & 84,7 & 84,80 & \multirow{3}{*}{4,17} & \multirow{3}{*}{$-0,33$} \\
\hline & $\mathbf{a}$ & 88,8 & 88,7 & 89,3 & 88,93 & 89,6 & 88,9 & 89,3 & 82,26 & 0,7 & 0,7 & 0,9 & 0,77 & 0,8 & 0,8 & 0,7 & 0,77 & & \\
\hline & b & 0,7 & 0,7 & 0,6 & 0,66 & 0,5 & 0,6 & 0,6 & 0,56 & 26,4 & 26,1 & 26,4 & 26,30 & 26,4 & 26,1 & 26,2 & 26,23 & & \\
\hline \multirow[t]{3}{*}{5} & $\mathbf{L}$ & 26,3 & 26,5 & 26 & 26,26 & 25,9 & 26,2 & 26,1 & 26,06 & 86 & 85,7 & 86,1 & 85,93 & 86,1 & 86,3 & 86,6 & 86,33 & \multirow{3}{*}{3,30} & \multirow{3}{*}{0,25} \\
\hline & $\mathbf{a}$ & 89,2 & 89,2 & 89,3 & 89,23 & 89,2 & 89,2 & 89,2 & 89,2 & $-0,3$ & $-0,2$ & $-0,2$ & $-0,23$ & $-0,2$ & $-0,3$ & $-0,3$ & $-0,27$ & & \\
\hline & b & $-0,2$ & $\begin{array}{l}-0,2 \\
\end{array}$ & $\begin{array}{l}-0,3 \\
\end{array}$ & $-0,23$ & $\begin{array}{l}-0,2 \\
\end{array}$ & $-0,3$ & $\begin{array}{l}-0,2 \\
\end{array}$ & $-0,23$ & 23,6 & 24,1 & 24 & 23,90 & 24,1 & 24 & 24 & 24,03 & & \\
\hline 6 & $\mathbf{L}$ & 24,1 & 24,1 & 23,9 & 24,03 & 24,1 & 23,4 & 24,1 & 23,86 & 89,2 & 89,6 & 89,1 & 89,30 & 89,3 & 89,7 & 89,5 & 89,50 & & \\
\hline & $\mathbf{a}$ & 91,6 & 91,5 & 91,5 & 91,53 & 91,5 & 91,4 & 91,4 & 91,43 & $-0,2$ & $-0,2$ & $-0,1$ & $-0,17$ & $-0,2$ & $-0,1$ & $-0,1$ & $-0,13$ & 2,25 & 0,12 \\
\hline & b & 0 & 0 & 0 & 0 & $-0,1$ & 0 & 0 & $-0,03$ & 24,2 & 24,7 & 24,5 & 24,47 & 24,5 & 24,5 & 24,7 & 24,57 & & \\
\hline 7 & $L$ & 24,1 & 24,4 & 24,4 & 24,3 & 24,3 & 24,3 & 24,4 & 24,33 & 88,4 & 87,9 & 87,7 & 88,00 & 88 & 87,9 & 87,7 & 87,87 & & \\
\hline & $\mathbf{a}$ & 90,9 & 90,8 & 91,1 & 90,93 & 90,9 & 90,9 & 90,9 & 90,9 & $-0,4$ & $-0,5$ & $-0,3$ & $-0,40$ & $-0,4$ & $-0,4$ & -03 & $-0,37$ & 3,01 & $-0,02$ \\
\hline & b & $-0,7$ & $-0,7$ & $-0,8$ & $-0,73$ & $-0,7$ & $-0,7$ & $-0,6$ & $-0,66$ & 24,1 & 23,7 & 24 & 23,93 & 24 & 24,1 & 24,1 & 24,07 & & \\
\hline 8 & $\mathbf{L}$ & 23,5 & 23,3 & 23,2 & 23,33 & 23,4 & 23,6 & 23,6 & 23,53 & 87,3 & 87,2 & 87,2 & 87,23 & 87,2 & 87,9 & 87,2 & 87,43 & & \\
\hline & $\mathbf{a}$ & 90,4 & 90,5 & 90,6 & 90,5 & 90,6 & 90,5 & 90,6 & 90,56 & 0,2 & 0 & 0 & 0,07 & 0 & 0,2 & 0,1 & 0,10 & 3,44 & 0,11 \\
\hline & b & $-0,3$ & $-0,3$ & $-0,3$ & $-0,3$ & $-0,3$ & $-0,3$ & $-0,3$ & $-0,3$ & 24,9 & 24,8 & 24,8 & 24,83 & 24,6 & 25,2 & 24,8 & 24,87 & & \\
\hline 9 & L & 23,9 & 23,9 & 23,7 & 23,83 & 23,9 & 23,9 & 23,9 & 23,9 & 87,9 & 87,8 & 87,9 & 87,87 & 87,8 & 88,2 & 88,2 & 88,07 & & \\
\hline & $\mathbf{a}$ & 91,6 & 91,6 & 91,7 & 91,63 & 91,8 & 91,7 & 91,7 & 91,73 & 0,3 & 0,4 & 0,4 & 0,37 & 0,3 & 0,3 & 0,3 & 0,30 & 3,77 & 0,11 \\
\hline & b & 0,2 & 0,2 & 0,1 & 0,16 & 0,2 & 0,1 & 0,1 & 0,13 & 24,9 & 24,9 & 24,6 & 24,80 & 24,6 & 24,9 & 24,8 & 24,77 & & \\
\hline & L & 24,9 & 25 & 24,6 & 24,83 & 25 & 24,7 & 24,8 & 24,83 & 88,4 & 88,3 & 88,8 & 88,50 & 88,7 & 88,8 & 88,9 & 88,80 & & \\
\hline 10 & $a$ & 91,7 & 91,2 & 91 & 91,3 & 90,8 & 91,5 & 91,2 & 91,16 & 0,1 & 0,2 & 0,2 & 0,17 & 0,1 & 0,1 & 0,2 & 0,13 & 2,82 & 0,18 \\
\hline & b & 0,4 & 0,5 & 0,5 & 0,46 & 0,4 & 0,4 & 0,5 & 0,43 & 26,7 & 26,8 & 27,1 & 26,87 & 27 & 27 & 27 & 27,00 & & \\
\hline
\end{tabular}


Quadro 9- Leituras de cor das amostras do Cimento fotoativado (grupo controle).

\begin{tabular}{|c|c|c|c|c|c|c|c|c|c|c|c|c|c|c|c|c|c|c|c|}
\hline \multicolumn{20}{|c|}{$\begin{array}{c}\text { Controle Variolink II } \\
\end{array}$} \\
\hline \multirow{2}{*}{$\begin{array}{c}\text { Corpo de } \\
\text { Prova }\end{array}$} & \multirow{2}{*}{ EIXO } & \multicolumn{4}{|c|}{ LEITURA INICIA Branco } & \multicolumn{4}{|c|}{ LEITURA INICIAL Preto } & \multicolumn{4}{|c|}{ LEITURA Final Branco } & \multicolumn{4}{|c|}{ LEITURA FINAL Preto } & \multicolumn{2}{|c|}{$\Delta$} \\
\hline & & Ll1 & $\mathrm{L} 12$ & $\mathrm{LI} 3$ & MEDIA LI & LF1 & LF2 & LF3 & MEDIA LF & LI1 & $\mathrm{LI} 2$ & LI3 & MEDIA LI & LF1 & LF2 & LF3 & MEDIA LF & $\Delta \mathrm{E}$ & $\Delta \mathbf{T}$ \\
\hline \multirow[t]{3}{*}{1} & $\mathbf{L}$ & 99,7 & 99,9 & 100 & 99,86 & 76,9 & 77 & 77 & 76,96 & 99 & 99,2 & 99,1 & 99,10 & 77,9 & 70,1 & 78,1 & 75,37 & \multirow{3}{*}{ 3,21 } & \multirow{3}{*}{0,64} \\
\hline & $\mathbf{a}$ & $-5,7$ & $-5,7$ & $-5,7$ & $-5,7$ & $-5,2$ & $-5,3$ & $-5,3$ & $-5,26$ & $-3,7$ & $\begin{array}{l}3,7 \\
\end{array}$ & $\begin{array}{l}-3,6 \\
\end{array}$ & $\begin{array}{l}-3,67 \\
\end{array}$ & $\begin{array}{l}-3,9 \\
\end{array}$ & -4 & $\begin{array}{l}-3,9 \\
\end{array}$ & $\begin{array}{l}-3,93 \\
\end{array}$ & & \\
\hline & b & 13 & 13 & 13,1 & 13,03 & 3,2 & 3,2 & 3,2 & 3,2 & 15,2 & 15,5 & 15,5 & 15,40 & 5,7 & 6 & 6 & 5,90 & & \\
\hline \multirow[t]{3}{*}{2} & $\mathbf{L}$ & 100,2 & 100,2 & 100,2 & 100,2 & 76,2 & 76,3 & 76,3 & 76,26 & 98,1 & 98,1 & 98,2 & 98,13 & 76 & 75,9 & 76,1 & 76,00 & \multirow{3}{*}{2,82} & \multirow{3}{*}{$-1,81$} \\
\hline & $a$ & $-5,7$ & $-5,7$ & $-5,7$ & $-5,7$ & $\begin{array}{l}-5,2 \\
\end{array}$ & $-5,1$ & $-5,2$ & $-5,16$ & $-4,1$ & $-4,1$ & -4 & $-4,07$ & $-4,2$ & $-4,1$ & $-4,1$ & $-4,13$ & & \\
\hline & b & 12,6 & 12,5 & 12,6 & 12,56 & 3 & 3 & 3,1 & 3,03 & 13,5 & 135 & 13,7 & 13,57 & 4,3 & 4,4 & 4,5 & 4,40 & & \\
\hline \multirow[t]{3}{*}{3} & $\mathbf{L}$ & 99,6 & 99,8 & 99,7 & 99,7 & 76 & 75,9 & 75,9 & 75,93 & 98,5 & 98,6 & 99,3 & 98,80 & 77,4 & 77,6 & 77,5 & 77,50 & \multirow{3}{*}{3,10} & \multirow{3}{*}{$-2,49$} \\
\hline & $a$ & $-5,8$ & $-5,8$ & $-5,8$ & $-5,8$ & $-5,3$ & $-5,3$ & $-5,3$ & $-5,3$ & $-4,1$ & $-3,9$ & $-3,9$ & $-3,97$ & $-4,2$ & $-4,2$ & $\begin{array}{l}-4,1 \\
\end{array}$ & $-4,17$ & & \\
\hline & b & 12,3 & 12,3 & 12,4 & 12,33 & 2,7 & 2,9 & 2,7 & 2,76 & 14,1 & 14,7 & 15,2 & 14,67 & 5,5 & 5,6 & 5,8 & 5,63 & & \\
\hline \multirow[t]{3}{*}{4} & $\mathbf{L}$ & 100,2 & 100,1 & 100 & 100,1 & 76,1 & 75,8 & 76 & 75,96 & 98,7 & 98,9 & 99 & 98,87 & 77,3 & 77,4 & 77,4 & 77,37 & \multirow{3}{*}{3,40} & \multirow{3}{*}{$-2,80$} \\
\hline & $\mathbf{a}$ & $-5,9$ & $-5,8$ & $-5,8$ & $-5,83$ & $-5,3$ & $-5,3$ & $-5,3$ & $-5,3$ & -4 & -4 & $\begin{array}{l}-3,8 \\
\end{array}$ & $\begin{array}{l}-3,93 \\
\end{array}$ & -4 & -4 & -4 & $-4,00$ & & \\
\hline & b & 12,3 & 12,3 & 12,2 & 12,26 & 2,9 & 2,6 & 2,6 & 2,7 & 14,3 & 14,9 & 15,2 & 14,80 & 6 & 6,2 & 6,3 & 6,17 & & \\
\hline \multirow[t]{3}{*}{5} & $\mathbf{L}$ & 100,3 & 100,4 & 100,6 & 100,43 & 76 & 75,9 & 76,2 & 76,03 & 99,5 & 99,4 & 99,4 & 99,43 & 77,1 & 77,5 & 77,7 & 77,43 & \multirow{3}{*}{3,45} & \multirow{3}{*}{$-2,53$} \\
\hline & $a$ & $-5,8$ & $-5,8$ & $-5,8$ & $-5,8$ & $-5,3$ & $-5,3$ & $-5,3$ & $-5,3$ & $-3,9$ & $-0,8$ & $-3,7$ & $-3,80$ & $-3,8$ & $-3,7$ & $-3,8$ & $-3,77$ & & \\
\hline & b & 12,4 & 12,5 & 12,4 & 12,43 & 2,7 & 2,7 & 2,7 & 2,7 & 15 & 15 & 15,2 & 15,07 & 5,9 & 6,3 & 6,2 & 6,13 & & \\
\hline 6 & $\mathbf{L}$ & 99,3 & 99,5 & 99,5 & 99,43 & 75,8 & 75,5 & 75,6 & $\begin{array}{l}75,63 \\
\end{array}$ & 98,7 & 99,3 & 99,4 & 99,13 & 77,3 & 77,5 & 77,5 & 77,43 & & \\
\hline & $\mathbf{a}$ & -6 & -6 & -6 & -6 & $-5,6$ & $-5,5$ & $-5,5$ & $-5,53$ & $-4,2$ & $-4,1$ & $-4,1$ & $-4,13$ & $-4,2$ & $-4,2$ & $-4,2$ & $-4,20$ & 3,30 & $-2,24$ \\
\hline & b & 11,6 & 11,7 & 11,7 & 11,66 & 2,2 & 2,1 & 2,2 & 2,16 & 13,7 & 14,7 & 14,7 & 14,37 & 5,5 & 5,6 & 5,8 & 5,63 & & \\
\hline 7 & $\mathbf{L}$ & 99,7 & 99,7 & 99,6 & 99,66 & 76,3 & 76,3 & 76,1 & 76,23 & 98,9 & 98,9 & 99,2 & 99,00 & 77 & 77,3 & 77,4 & 77,23 & & \\
\hline & $a$ & $-5,8$ & $-5,8$ & $-5,7$ & $-5,76$ & $\begin{array}{l}-5,3 \\
\end{array}$ & $-5,3$ & $-5,2$ & $-5,26$ & $-4,4$ & $\begin{array}{l}-4,4 \\
\end{array}$ & $-4,4$ & $-4,40$ & $-4,3$ & $\begin{array}{l}-4,3 \\
\end{array}$ & $\begin{array}{l}-4,3 \\
\end{array}$ & $\begin{array}{l}-4,30 \\
\end{array}$ & 1,82 & $-1,68$ \\
\hline & b & 12,3 & 12,3 & 12,4 & 12,33 & 3,1 & 3,2 & 3,1 & 3,13 & 13,2 & 13,3 & 13,5 & 13,33 & 4,3 & 4,5 & 4,6 & 4,47 & & \\
\hline 8 & $\mathbf{L}$ & 99 & 98,9 & 98,8 & 98,9 & 75 & 74,7 & 74,9 & 74,86 & 99,2 & 99,1 & 99 & 99,10 & 77,5 & 77,7 & 77,6 & 77,60 & & \\
\hline & $a$ & $-5,6$ & $-5,7$ & $-5,7$ & $-5,66$ & $-5,2$ & $-5,2$ & $-5,2$ & $-5,2$ & -4 & $\begin{array}{l}-3,9 \\
\end{array}$ & $-3,8$ & $-3,90$ & -4 & $-3,9$ & $\begin{array}{l}-3,9 \\
\end{array}$ & $\begin{array}{l}-3,93 \\
\end{array}$ & 4,65 & $-2,52$ \\
\hline & b & 11 & 11 & 11 & 11 & 1,8 & 1,7 & 1,7 & 1,73 & 15 & 15,5 & 15,4 & 15,30 & 6,4 & 6,5 & 6,5 & 6,47 & & \\
\hline 9 & $\mathbf{L}$ & 100 & 99,8 & 99,9 & 99,9 & 76,1 & 76,1 & 76,1 & 76,1 & 101 & 101,3 & 100,9 & 101,07 & 79,3 & 79,7 & 79,2 & 79,40 & & \\
\hline & $a$ & $-5,6$ & $-5,6$ & $-5,6$ & $-5,6$ & $-5,2$ & $-5,2$ & $-5,1$ & $-5,26$ & -4 & -4 & -4 & $-4,00$ & $-4,3$ & $-4,3$ & $-4,3$ & $-4,30$ & 3,79 & $-1,90$ \\
\hline & b & 12,2 & 12,4 & 12,1 & 12,23 & 2,8 & 2,8 & 2,8 & 2,8 & 15,4 & 15,5 & 15,5 & 15,47 & 5,8 & 6 & 58 & 5,87 & & \\
\hline & $\mathbf{L}$ & 98,9 & 98,8 & 98,8 & 98,83 & 74,5 & 74,5 & 74,6 & 74,53 & 100,7 & 101,7 & 101,7 & 101,37 & 78,7 & 78,8 & 78,7 & 78,73 & & \\
\hline 10 & $a$ & $-5,7$ & $-5,6$ & $-5,6$ & $-5,63$ & $-5,2$ & $-5,3$ & $-5,3$ & $-5,26$ & $-4,7$ & $-4,1$ & $\begin{array}{l}-4,2 \\
\end{array}$ & $\begin{array}{l}-4,33 \\
\end{array}$ & $\begin{array}{l}-4,2 \\
\end{array}$ & $-4,2$ & $\begin{array}{l}-4,2 \\
\end{array}$ & $-4,20$ & 5,19 & $-1,73$ \\
\hline & b & 10,7 & 10,7 & 10,7 & 10,7 & 1,5 & 1,5 & 1,5 & 1,5 & 13,6 & 15,7 & 15,8 & 15,03 & 6,1 & 6,5 & 6,3 & 6,30 & & \\
\hline
\end{tabular}


Quadro 10- Leituras de cor das amostras do Cimento autoadesivo (grupos controle).

\begin{tabular}{|c|c|c|c|c|c|c|c|c|c|c|c|c|c|c|c|c|c|c|c|}
\hline \multicolumn{20}{|c|}{ Controle Rely-X U200 } \\
\hline \multirow{2}{*}{$\begin{array}{c}\text { Corpo de } \\
\text { Prova }\end{array}$} & \multirow{2}{*}{ EIXO } & \multicolumn{4}{|c|}{ LEITURA INICIA Branco } & \multicolumn{4}{|c|}{ LEITURA INICIAL Preto } & \multicolumn{4}{|c|}{ LEITURA Final Branco } & \multicolumn{4}{|c|}{ LEITURA FINAL Preto } & \multicolumn{2}{|c|}{$\Delta$} \\
\hline & & LI1 & LI2 & LI3 & MEDIA LI & LF1 & LF2 & LF3 & MEDIA LF & LI1 & LI2 & LI3 & MEDIA LI & LF1 & LF2 & LF3 & MEDIA LF & $\Delta \mathrm{E}$ & $\Delta T$ \\
\hline \multirow[t]{3}{*}{1} & $\mathbf{L}$ & 102,3 & 102,2 & 102,2 & 102,23 & 79,5 & 79 & 79 & 79,16 & 94,9 & 95,1 & 95,2 & 95,07 & 75,9 & 75,8 & 76,3 & 76,00 & \multirow{3}{*}{28,25} & \multirow{3}{*}{$-0,77$} \\
\hline & $\mathbf{a}$ & $-4,5$ & $-4,4$ & $-4,4$ & $-4,43$ & $-3,6$ & $-3,5$ & $-3,5$ & $-3,53$ & $-1,9$ & $-1,7$ & $-1,8$ & $-1,80$ & $-4,1$ & $-4,1$ & $-4,1$ & $-4,10$ & & \\
\hline & b & 14,9 & 14,9 & 14,9 & 14,9 & 5,7 & 5,6 & 5,6 & 5,63 & 41,9 & 42,3 & 42,1 & 42,10 & 27,4 & 27,5 & 27,7 & 27,53 & & \\
\hline \multirow[t]{3}{*}{2} & $\mathbf{L}$ & 98,8 & 98,8 & 98,5 & 98,7 & 75,2 & 75,1 & 75,4 & 75,23 & 91,2 & 91,5 & 91,6 & 91,43 & 71,9 & 72,3 & 72,9 & 72,37 & \multirow{3}{*}{30,86} & \multirow{3}{*}{$-1,13$} \\
\hline & $\mathbf{a}$ & $-4,9$ & $-4,8$ & $-4,8$ & $\begin{array}{l}-4,83 \\
\end{array}$ & -4 & $-3,9$ & -4 & $-3,96$ & $-1,9$ & $-1,8$ & $-1,7$ & $-1,80$ & $-4,4$ & $-4,3$ & $-4,3$ & $\begin{array}{l}-4,33 \\
\end{array}$ & & \\
\hline & b & 13,2 & 13,2 & 13,2 & 13,2 & 3,5 & 3,6 & 3,5 & 3,53 & 42,6 & 43,2 & 43,3 & 43,03 & 28 & 28,3 & 28,4 & 28,23 & & \\
\hline \multirow[t]{3}{*}{3} & $\mathbf{L}$ & 97,1 & 96,8 & 97,2 & 97,03 & 72,3 & 72,5 & 72,6 & 72,46 & 92,4 & 92,2 & 92,4 & 92,33 & 72,2 & 72,5 & 72,7 & 72,47 & \multirow{3}{*}{27,82} & \multirow{3}{*}{$-1,88$} \\
\hline & $a$ & -5 & -5 & $-5,1$ & $-5,03$ & $-4,2$ & $-4,2$ & $-4,2$ & $-4,2$ & $-3,3$ & $-3,3$ & $-3,2$ & $-3,27$ & $-5,3$ & $-5,2$ & $-5,2$ & $-5,23$ & & \\
\hline & b & 10,8 & 10,8 & 10,9 & 10,83 & 0,8 & 0,8 & 0,9 & 0,83 & 38,1 & 38,3 & 38,2 & 38,20 & 23,4 & 23,9 & 23,9 & 23,73 & & \\
\hline \multirow[t]{3}{*}{4} & L & 100,9 & 100 & 100,1 & 100,33 & 75,3 & 75,4 & 75,3 & 75,33 & 93,7 & 94 & 94,3 & 94,00 & 73,8 & 737 & 73,8 & 73,77 & \multirow{3}{*}{30,80} & \multirow{3}{*}{$-1,25$} \\
\hline & $a$ & -5 & $-4,9$ & -5 & $-4,96$ & -4 & -4 & -4 & -4 & -2 & -2 & -2 & $-2,00$ & $-4,4$ & $-4,3$ & $-4,3$ & \begin{tabular}{|l|}
$-4,33$ \\
\end{tabular} & & \\
\hline & b & 12,7 & 12,6 & 12,6 & 12,63 & 2,8 & 2,9 & 3 & 2,9 & 42,4 & 42,7 & 42,8 & 42,63 & 27 & 27,2 & 27,2 & 27,13 & & \\
\hline \multirow[t]{3}{*}{5} & L & 101 & 100,9 & 100,9 & 100,93 & 77,3 & 77,3 & 77,5 & 77,36 & 94,5 & 94 & 93,7 & 94,00 & 74,8 & 74,8 & 74,1 & 74,57 & \multirow{3}{*}{31,66} & \multirow{3}{*}{$-0,37$} \\
\hline & $a$ & $-4,9$ & $-4,8$ & $-4,8$ & $-4,83$ & -4 & $-4,1$ & $-4,1$ & $-4,06$ & $-1,4$ & $-1,3$ & -1 & $-1,23$ & $-4,1$ & -4 & $-3,8$ & $-3,97$ & & \\
\hline & b & 13,1 & 13,6 & 13,6 & 13,43 & 4,3 & 4,3 & 4,3 & 4,3 & 43,9 & 44,1 & 44,4 & 44,13 & 28,8 & 29 & 28,8 & 28,87 & & \\
\hline 6 & $\mathbf{L}$ & 100 & 99,7 & 99,8 & 99,83 & 76,5 & 77 & 76,7 & 76,73 & 91,1 & 91,8 & 92,8 & 91,90 & 72,1 & 72,2 & 72,4 & 72,23 & & \\
\hline & a & $-4,5$ & $-4,6$ & $-4,6$ & $-4,56$ & -3 & $-3,7$ & $-3,7$ & $-3,46$ & $-1,7$ & $-1,4$ & $-1,3$ & $-1,47$ & -4 & -4 & -4 & $-4,00$ & 31,51 & $-0,02$ \\
\hline & b & 13,2 & 13,6 & 13,3 & 13,36 & 3,8 & 4,1 & 4 & 3,96 & 43,1 & 43,8 & 44,2 & 43,70 & 28,6 & 28,5 & 28,6 & 28,57 & & \\
\hline 7 & L & 98,9 & 98,2 & 98,4 & 98,46 & 74,1 & 73,9 & 74,2 & 74,06 & 92,8 & 93,2 & 93,4 & 93,13 & 72,8 & 72,5 & 72,7 & 72,67 & & \\
\hline & $a$ & $-5,1$ & $-5,3$ & $-5,1$ & $-5,16$ & $-4,3$ & $-4,3$ & $-4,3$ & $-4,3$ & $-3,1$ & $-2,9$ & $-2,8$ & $-2,93$ & $-4,9$ & $-4,9$ & -5 & $-4,93$ & 29,67 & $-0,82$ \\
\hline & b & 11,1 & 10,9 & 11,2 & 11,06 & 1,3 & 1,4 & 1,4 & 1,36 & 39,8 & 40,3 & 40,4 & 40,17 & 25,2 & 25,1 & 25,2 & 25,17 & & \\
\hline 8 & $\mathbf{L}$ & 100,4 & 100,5 & 100,3 & 100,4 & 76 & 76,5 & 76,3 & 76,26 & 92,9 & 92,5 & 93,2 & 92,87 & 73,9 & 74 & 73,9 & 73,93 & & \\
\hline & a & $-4,8$ & $-4,8$ & $-4,8$ & $-4,8$ & -4 & $-3,9$ & -4 & $-3,9$ & $-1,7$ & $-1,5$ & $-1,6$ & $-1,60$ & $-4,1$ & $-4,1$ & $-4,1$ & $-4,10$ & 30,81 & $-1,72$ \\
\hline & b & 12,9 & 13 & 13 & 12,96 & 3,5 & 3,8 & 3,5 & 3,6 & 42,5 & 42,7 & 42,8 & 42,67 & 27,8 & 27,9 & 27,8 & 27,83 & & \\
\hline 9 & L & 99,6 & 99,6 & 99,7 & 99,63 & 75,2 & 75,3 & 75,4 & 75,3 & 93,4 & 93,6 & 93,9 & 93,63 & 73,3 & 73,5 & 73,6 & 73,47 & & \\
\hline & $a$ & $-5,2$ & $-5,2$ & $-5,2$ & $-5,2$ & $-4,4$ & $-4,3$ & $-4,3$ & $-4,33$ & $-3,3$ & $-3,1$ & -3 & $-3,13$ & $-5,1$ & $-5,1$ & -5 & $-5,07$ & 28,39 & $-1,16$ \\
\hline & b & 11,7 & 11,7 & 11,7 & 11,7 & 1,8 & 1,8 & 1,9 & 1,83 & 38,8 & 39,5 & 39,8 & 39,37 & 24,4 & 24,6 & 24,6 & 24,53 & & \\
\hline & $\mathbf{L}$ & 97,6 & 97,9 & 97,6 & 97,7 & 74,6 & 74,9 & 75,2 & 74,9 & 91,3 & 91,8 & 91,7 & 91,60 & 74,2 & 73,9 & 73,8 & 73,97 & & \\
\hline 10 & a & $-5,2$ & $-5,3$ & $-5,3$ & $-5,26$ & $-4,3$ & $-4,2$ & $-4,3$ & $-4,26$ & $-0,3$ & 0 & 0 & $-0,10$ & $-3,1$ & $-3,2$ & $-3,1$ & $-3,10$ & 35,93 & $-1,34$ \\
\hline & b & 10,6 & 10,5 & 10,6 & 10,56 & 2,2 & 2,3 & 2,5 & 2,33 & 45 & 46,1 & 45,7 & 45,60 & 31,4 & 31,2 & 31,2 & 31,27 & & \\
\hline
\end{tabular}


Quadro 11- Leituras de cor das amostras dos dentes (grupo controle)

\begin{tabular}{|c|c|c|c|c|c|c|c|c|c|c|c|c|c|c|c|c|c|c|c|}
\hline \multicolumn{20}{|c|}{ Controle Dentes } \\
\hline \multirow{2}{*}{$\begin{array}{c}\text { Corpo de } \\
\text { Prova }\end{array}$} & \multirow{2}{*}{ EIXO } & \multicolumn{4}{|c|}{ LEITURA INICIA Branco } & \multicolumn{4}{|c|}{ LEITURA INICIAL Preto } & \multicolumn{4}{|c|}{ LEITURA Final Branco } & \multicolumn{4}{|c|}{ LEITURA FINAL Preto } & \multicolumn{2}{|c|}{$\Delta$} \\
\hline & & LI1 & LI2 & LI3 & MEDIA LI & LF1 & LF2 & LF3 & MEDIA LF & LI1 & LI2 & LI3 & MEDIA LI & LF1 & LF2 & LF3 & MEDIA LF & $\Delta \mathrm{E}$ & $\Delta T$ \\
\hline \multirow[t]{3}{*}{1} & $\mathbf{L}$ & 90,6 & 91 & 90,8 & 90,8 & 90,3 & 89,9 & 89,7 & 89,96 & 81,5 & 81,6 & 80,8 & 81,30 & 80,7 & 80,1 & 81,2 & 80,67 & \multirow{3}{*}{11,32} & \multirow{3}{*}{$-0,28$} \\
\hline & a & $-0,2$ & $-0,2$ & $-0,2$ & $-0,2$ & $-0,2$ & $-0,3$ & $-0,3$ & $-0,26$ & 2,8 & 2,5 & 3,1 & 2,80 & 2,8 & 2,9 & 2,7 & 2,80 & & \\
\hline & b & 29,5 & 29,5 & 29,4 & 29,46 & 29,4 & 28,9 & 28,7 & 29 & 35,2 & 34,2 & 35,1 & 34,83 & 35,2 & 35 & 35 & 35,07 & & \\
\hline \multirow[t]{3}{*}{2} & $\mathbf{L}$ & 97,7 & 97 & 96,8 & 97,16 & 97,8 & 97,8 & 97,4 & 97,66 & 90,2 & 89,8 & 88,5 & 89,50 & 90,4 & 90,1 & 91,5 & 90,67 & \multirow{3}{*}{6,54} & \multirow{3}{*}{0,30} \\
\hline & $\mathbf{a}$ & 0 & $-0,1$ & $\begin{array}{l}-0,2 \\
\end{array}$ & $-0,1$ & $-0,2$ & $-0,2$ & $-0,2$ & $-0,2$ & 2,2 & 2 & 1,8 & 2,00 & 1,9 & 1,9 & 1,7 & 1,83 & & \\
\hline & b & 27,2 & 27,8 & 27,3 & 27,43 & 28,7 & 28,3 & 28 & 28,33 & 33,4 & 32,8 & 31,9 & 32,70 & 33,5 & 33,3 & 33,2 & 33,33 & & \\
\hline \multirow[t]{3}{*}{3} & $\mathbf{L}$ & 96,5 & 96,9 & 96,6 & 96,66 & 96,8 & 96,8 & 96,7 & 96,76 & 88,2 & 83,8 & 82,3 & 84,77 & 82,8 & 82,6 & 81,3 & 82,23 & \multirow{3}{*}{13,15} & \multirow{3}{*}{2,34} \\
\hline & $a$ & 0 & 0 & $-0,1$ & $-0,03$ & 0 & $-0,1$ & $-0,2$ & $-0,1$ & 3,1 & 3 & 3 & 3,03 & 3,2 & 3,2 & 3,1 & 3,17 & & \\
\hline & b & 30,8 & 31,7 & 30,3 & 30,93 & 31,1 & 30,6 & 30,2 & 30,63 & 37,2 & 35,1 & 34,5 & 35,60 & 35,5 & 35 & 33,9 & 34,80 & & \\
\hline \multirow[t]{3}{*}{4} & L & 89,2 & 89 & 89 & 89,06 & 90 & 89,1 & 90 & 89,7 & 83,7 & 84,7 & 84,3 & 84,23 & 84,1 & 84,2 & 83,8 & 84,00 & \multirow{3}{*}{6,23} & \multirow{3}{*}{$-0,34$} \\
\hline & $a$ & $-0,8$ & $-0,7$ & $-0,7$ & $\begin{array}{l}-0,73 \\
\end{array}$ & $\begin{array}{l}-0,8 \\
\end{array}$ & $\begin{array}{l}-0,8 \\
\end{array}$ & $-0,8$ & $-0,8$ & 0,4 & 0,2 & 0,3 & 0,30 & 0,4 & 0,4 & 0,5 & 0,43 & & \\
\hline & b & 26,6 & 26,4 & 27,2 & 26,73 & 27,4 & 26,4 & 26,9 & 26,9 & 30,4 & 30,9 & 30,3 & 30,53 & 30,2 & 30,4 & 30,5 & 30,37 & & \\
\hline \multirow[t]{3}{*}{5} & L & 99,6 & 99 & 99,6 & 99,4 & 99,2 & 99,5 & 99,2 & 99,3 & 89,3 & 88,3 & 88,4 & 88,67 & 88,2 & 88,5 & 88,3 & 88,33 & \multirow{3}{*}{10,80} & \multirow{3}{*}{$-0,19$} \\
\hline & $a$ & 0,6 & 0,5 & 0,6 & 0,56 & 0,6 & 0,6 & 0,7 & 0,63 & 1 & 1,1 & 1,3 & 1,13 & 1,1 & 1 & 1,2 & 1,10 & & \\
\hline & b & 29,5 & 29 & 29,6 & 29,36 & 29,6 & 29,9 & 30,3 & 29,93 & 30,3 & 30,3 & 30,7 & 30,43 & 30,1 & 30,1 & 30,5 & 30,23 & & \\
\hline 6 & $\mathbf{L}$ & 83,2 & 83,2 & 84,2 & 83,53 & 85,3 & 85,5 & 85,1 & 85,3 & 72,2 & 72,5 & 71,8 & 72,17 & 71,9 & 73,9 & 72,5 & 72,77 & & \\
\hline & a & $-0,4$ & $-0,4$ & $-0,4$ & $-0,4$ & $-0,5$ & $-0,5$ & $-0,5$ & $-0,5$ & 3,7 & 3,7 & 3,8 & 3,73 & 3,5 & 3,6 & 3,9 & 3,67 & 15,8 & $-1,53$ \\
\hline & b & 22,3 & 22,5 & 23,1 & 22,63 & 23,6 & 24,1 & 24 & 23,9 & 32,9 & 32,7 & 328 & 32,80 & 32,2 & 33,4 & 33,5 & 33,03 & & \\
\hline 7 & L & 92,2 & 92,4 & 92 & 92,2 & 92,5 & 92,8 & 92,2 & 92,5 & 82,1 & 82 & 81,4 & 81,83 & 81,4 & 81,4 & 81,3 & 81,37 & & \\
\hline & $a$ & 0,3 & 0,3 & 0,3 & 0,3 & 0,3 & 0,3 & 0,3 & 0,3 & 4,2 & 4 & 4,3 & 4,17 & 4,3 & 4,3 & 4,3 & 4,30 & 12,36 & $-0,73$ \\
\hline & b & 30,9 & 30,8 & 31,3 & 31 & 32 & 32,5 & 32,1 & 32,2 & 36,7 & 36,4 & 36,4 & 36,50 & 36,7 & 36,5 & 36,7 & 36,63 & & \\
\hline 8 & L & 90,2 & 89,4 & 87,7 & 89,1 & 84 & 86 & 86,3 & 85,43 & 84,3 & 84,3 & 84,4 & 84,33 & 84,2 & 84,2 & 84,4 & 84,27 & & \\
\hline & a & 0,4 & 0,5 & 0,6 & 0,5 & 0,8 & 0,7 & 0,3 & 0,6 & 0,8 & 0,8 & 0,8 & 0,80 & 0,7 & 0,8 & 0,8 & 0,77 & 4,94 & $-3,53$ \\
\hline & b & 30,6 & 30,7 & 31,1 & 30,8 & 31,7 & 31,7 & 31,7 & 31,7 & 29,5 & 29,7 & 29,4 & 29,53 & 29,2 & 29,3 & 29,4 & 29,30 & & \\
\hline 9 & L & 91 & 91,3 & 89,6 & 90,63 & 88,8 & 89,6 & 88,8 & 89,06 & 94,8 & 94,5 & 94,9 & 94,73 & 94,6 & 94,6 & 94,3 & 94,50 & & \\
\hline & $a$ & 1,3 & 1,3 & 1,4 & 1,33 & 1,3 & 1,4 & 1,4 & 1,36 & 2,3 & 2 & 2,2 & 2,17 & 2,2 & 2,3 & 2,2 & 2,23 & 4,33 & $-1,31$ \\
\hline & b & 33,9 & 33,9 & 34,5 & 34,1 & 34,7 & 34,6 & 34,5 & 34,6 & 35,5 & 34,7 & 35,5 & 35,23 & 35,6 & 35,7 & 35,1 & 35,47 & & \\
\hline & $\mathbf{L}$ & 85,8 & 85,5 & 85,6 & 85,63 & 84,7 & 85,2 & 85,4 & 85,1 & 82,6 & 83 & 82,6 & 82,73 & 82,3 & 82,7 & 81,9 & 82,30 & & \\
\hline 10 & a & 2,1 & 2,1 & 2,1 & 2,1 & 2,1 & 2 & 2 & 2,03 & 3,2 & 3,2 & 3,1 & 3,17 & 3,3 & 3,2 & 3,5 & 3,33 & 3,87 & $-0,25$ \\
\hline & b & 39,5 & 39,5 & 39,6 & 39,5 & 39,3 & 38,8 & 38,9 & 39 & 37,3 & 37,4 & 36,8 & 37,17 & 36,9 & 36,9 & 37,3 & 37,03 & & \\
\hline
\end{tabular}


Os quadros 12 ao 22 constam as leituras iniciais de cor, sobre fundo preto e fundo branco, dos corpos de prova que foram submetidos a fadiga termomecânica.

Quadro 12- Leituras de cor das amostras de E-max Press na espessura de 0,5mm cimentados com cimento fotoativado.

\begin{tabular}{|c|c|c|c|c|c|c|c|c|c|c|c|c|c|c|c|c|c|c|c|}
\hline \multicolumn{20}{|c|}{ E-max Press 0,5mm Variolink II } \\
\hline \multirow{2}{*}{$\begin{array}{c}\text { Corpo } \\
\text { de Prova }\end{array}$} & \multirow{2}{*}{ EIXO } & \multicolumn{4}{|c|}{ LEITURA INICIA Branco } & \multicolumn{4}{|c|}{ LEITURA INICIAL Preto } & \multicolumn{4}{|c|}{ LEITURA Final Branco } & \multicolumn{4}{|c|}{ LEITURA FINAL Preto } & \multicolumn{2}{|c|}{$\Delta$} \\
\hline & & LI1 & Ll2 & LI3 & MEDIA LI & LF1 & LF2 & LF3 & MEDIA LF & LI1 & LI2 & $\mathrm{LI} 3$ & MEDIA LI & LF1 & LF2 & LF3 & MEDIA LF & $\Delta \mathrm{E}$ & $\Delta \mathrm{T}$ \\
\hline \multirow{3}{*}{1} & $\mathbf{L}$ & 92,5 & 92,2 & 92,9 & 92,53 & 92,4 & 92,6 & 92,8 & 92,60 & & & & & & & & & & \\
\hline & $\mathbf{a}$ & $-1,8$ & $-1,8$ & $-1,8$ & $-1,8$ & $-1,8$ & $-1,8$ & $-1,8$ & $-1,80$ & & & & & & & & & & \\
\hline & b & 15,9 & 16 & 15,8 & 15,9 & 16 & 15,9 & 15,7 & 15,87 & & & & & & & & & & \\
\hline \multirow{3}{*}{2} & $\mathbf{L}$ & 94,7 & 94,5 & 94,9 & 94,70 & 94,6 & 94,6 & 94,8 & 94,67 & & & & & & & & & & \\
\hline & $\mathbf{a}$ & $-0,6$ & $-0,6$ & $-0,6$ & $-0,6$ & $-0,6$ & $-0,5$ & $-0,6$ & $-0,57$ & & & & & & & & & & \\
\hline & b & 24,1 & 23,5 & 23,6 & 23,73 & 24,5 & 24,3 & 24,2 & 24,33 & & & & & & & & & & \\
\hline \multirow{3}{*}{3} & $\mathbf{L}$ & 94,7 & 95,5 & 94,7 & 94,97 & 94,9 & 94,8 & 95,3 & 95,00 & 93,3 & 93 & 93,7 & 93,33 & 93,2 & 93,4 & 93,5 & 93,37 & & \\
\hline & $a$ & $-1,7$ & $-1,7$ & $-1,6$ & $-1,67$ & $-1,7$ & $-1,7$ & $-1,7$ & $-1,70$ & $-1,1$ & -1 & $-1,1$ & $-1,07$ & $-1,1$ & $-1,1$ & $-1,2$ & $-1,13$ & 1,81 & 0,04 \\
\hline & b & 19 & 18,9 & 19,2 & 19,03 & 19 & 18,7 & 19 & 18,90 & 19,5 & 19,2 & 19,9 & 19,53 & 19,9 & 19,8 & 19,4 & 19,70 & & \\
\hline \multirow{3}{*}{4} & L & 98,7 & 98,1 & 99,1 & 98,63 & 98,7 & 99,1 & 98,4 & 98,73 & 95,5 & 95,4 & 96,2 & 95,7 & 95,8 & 95,3 & 95,1 & 95,40 & & \\
\hline & a & $-0,5$ & $-0,4$ & $-0,5$ & $-0,47$ & $-0,4$ & $-0,5$ & $-0,4$ & $-0,43$ & $-0,2$ & $-0,1$ & $-0,3$ & $-0,20$ & $-0,2$ & $-0,1$ & $-0,1$ & $-0,13$ & 3,02 & 0,46 \\
\hline & b & 23,9 & 24 & 24 & 23,97 & 24,1 & 24,1 & 23,9 & 24,03 & 23,3 & 23,4 & 23,2 & 23,30 & 23,4 & 23,9 & 24,1 & 23,8 & & \\
\hline \multirow{3}{*}{5} & $\mathbf{L}$ & 96,3 & 96,5 & 96,7 & 96,50 & 96,5 & 96,6 & 96,4 & 96,50 & & & & & & & & & & \\
\hline & a & $-1,5$ & $-1,5$ & $-1,5$ & $-1,50$ & $-1,6$ & $-1,6$ & $-1,6$ & $-1,60$ & & & & & & & & & & \\
\hline & b & 21 & 21,2 & 21,3 & 21,17 & 20,3 & 204 & 20,5 & 20,40 & & & & & & & & & & \\
\hline \multirow{3}{*}{6} & L & 92 & 91,6 & 92 & 91,87 & 91,8 & 91,9 & 91,9 & 91,87 & 91 & 91,1 & 90,8 & 90,97 & 91,2 & 91,2 & 91,3 & 91,23 & & \\
\hline & $\mathbf{a}$ & 0,3 & 0,3 & 0,3 & 0,30 & 0,3 & 0,3 & 0,3 & 0,30 & 0,2 & 0,1 & 0,2 & 0,17 & 0,2 & 0,2 & 0,1 & 0,17 & 1,17 & $-0,13$ \\
\hline & b & 26,1 & 26,1 & 26,1 & 26,10 & 25,3 & 25,9 & 25,9 & 25,70 & 25,3 & 25,4 & 25,4 & 25,37 & 25,3 & 25,3 & 25,5 & 25,37 & & \\
\hline \multirow{3}{*}{7} & $\mathrm{~L}$ & 99,3 & 98,9 & 99,1 & 99,07 & 99,6 & 99 & 99,2 & 99,27 & & & & & & & & & & \\
\hline & $a$ & $-1,1$ & $-1,2$ & $-1,3$ & $-1,20$ & $-1,3$ & $-1,1$ & $-1,3$ & $-1,23$ & & & & & & & & & & \\
\hline & b & 21,2 & 20,7 & 20,4 & 20,77 & 20,3 & 21,2 & 20,3 & 20,60 & & & & & & & & & & \\
\hline \multirow{3}{*}{8} & $L$ & 98,9 & 98,4 & 99,1 & 98,77 & 98,6 & 98,1 & 99,3 & 98,67 & 93,5 & 94,5 & 94,7 & 94,23 & 94,5 & 94,4 & 93,8 & 94,23 & & \\
\hline & $\mathbf{a}$ & 0 & 0 & 0 & 0 & $-0,1$ & $-0,1$ & $-0,1$ & $-0,10$ & $-0,2$ & $-0,3$ & $-0,3$ & $-0,27$ & $-0,4$ & $-0,4$ & $-0,3$ & $-0,37$ & 5,22 & $-0,26$ \\
\hline & b & 27,2 & 26,8 & 27,1 & 27,03 & 26,5 & 26,5 & 26,8 & 26,60 & 24,6 & 24,3 & 24,5 & 24,47 & 24,4 & 24,3 & 24,2 & 24,30 & & \\
\hline \multirow{3}{*}{9} & $\mathbf{L}$ & 101,7 & 101,9 & 101,7 & 101,77 & 103,5 & 102,3 & 101,8 & 102,53 & 95,7 & 95,8 & 96,1 & 95,87 & 103,5 & 102,3 & 101,8 & 102,53 & & \\
\hline & $\mathbf{a}$ & $-0,6$ & $-0,8$ & $-0,8$ & $-0,73$ & $-0,9$ & $-0,8$ & $-0,7$ & $-0,80$ & $-1,2$ & $-1,2$ & $-1,3$ & $-1,23$ & $-0,9$ & $-0,8$ & $-0,7$ & $-0,80$ & 6,67 & $-0,43$ \\
\hline & b & 24,5 & 24,8 & 24,7 & 24,67 & 24,1 & 24,5 & 24,7 & 24,43 & 21,8 & 21,5 & 21,5 & 21,60 & 24,1 & 24,5 & 247 & 24,43 & & \\
\hline \multirow{3}{*}{10} & $\mathbf{L}$ & 97,3 & 98,1 & 98 & 97,80 & 97,6 & 98,2 & 97,6 & 97,80 & 93,6 & 93,6 & 93,6 & 93,60 & 97,6 & 98,2 & 97,6 & 97,80 & \multirow{3}{*}{4,68} & \multirow{3}{*}{0,08} \\
\hline & $\mathbf{a}$ & $-0,7$ & $-0,8$ & $-0,8$ & $-0,77$ & $-0,7$ & $-0,7$ & $-0,7$ & $-0,70$ & $-0,8$ & $-0,8$ & $-0,8$ & $-0,80$ & $-0,7$ & $-0,7$ & $-0,7$ & $-0,70$ & & \\
\hline & b & 21,5 & 21,2 & 21,3 & 21,33 & 21,2 & 21,2 & 21,2 & 21,20 & 19,1 & 19,3 & 19,4 & 19,27 & 21,2 & 21,2 & 21,2 & 21,20 & & \\
\hline
\end{tabular}


Quadro 13- Leituras de cor das amostras de E-max Press na espessura de 1,0mm cimentados com cimento fotoativado.

\begin{tabular}{|c|c|c|c|c|c|c|c|c|c|c|c|c|c|c|c|c|c|c|c|}
\hline \multicolumn{20}{|c|}{ E-max Press 1,0mm Variolink II } \\
\hline \multirow{2}{*}{$\begin{array}{l}\text { Corpo de } \\
\text { Prova }\end{array}$} & \multirow{2}{*}{ EIXO } & \multicolumn{4}{|c|}{ LEITURA INICIA Branco } & \multicolumn{4}{|c|}{ LEITURA INICIAL Preto } & \multicolumn{4}{|c|}{ LEITURA Final Branco } & \multicolumn{4}{|c|}{ LEITURA FINAL Preto } & \multicolumn{2}{|c|}{$\Delta$} \\
\hline & & LI1 & $\mathrm{LI} 2$ & LI3 & MEDIA LI & LF1 & LF2 & LF3 & MEDIA LF & Ll1 & $\mathrm{Ll} 2$ & LI3 & MEDIA LI & LF1 & LF2 & LF3 & MEDIA LF & $\Delta \mathrm{E}$ & $\Delta \mathrm{T}$ \\
\hline \multirow[t]{3}{*}{1} & $\mathbf{L}$ & 91,6 & 91,2 & 91,8 & 91,53 & 92,2 & 92,3 & 92 & 92,17 & 92 & 92,1 & 91,9 & 92,00 & 92,2 & 92,1 & 92,5 & 92,27 & \multirow{3}{*}{0,83} & \multirow{3}{*}{$-0,32$} \\
\hline & $\mathbf{a}$ & -1 & $-0,9$ & -1 & $-0,97$ & -1 & -1 & -1 & $-1,00$ & $-1,3$ & $-1,3$ & $-1,3$ & $-1,30$ & $-1,3$ & $-1,3$ & $-1,3$ & $-1,30$ & & \\
\hline & b & 18,6 & 18,6 & 18,5 & 18,57 & 18.5 & 18.6 & 18.6 & 18.57 & 19,5 & 18,9 & 19,1 & 19,17 & 19 & 19 & 19 & 19,00 & & \\
\hline \multirow[t]{3}{*}{2} & $\mathbf{L}$ & 93,3 & 93,5 & 94 & 93,6 & 93,6 & 93,7 & 93,7 & 93,67 & 92,6 & 92,9 & 93,2 & 92,90 & 93,3 & 93,3 & 93,5 & 93,37 & \multirow{3}{*}{0,86} & \multirow{3}{*}{0,49} \\
\hline & $\mathbf{a}$ & $-1,1$ & $-1,2$ & $-1,2$ & $-1,17$ & $-1,1$ & $-1,1$ & $-1,1$ & $-1,10$ & $-1,5$ & $-1,5$ & $-1,5$ & $-1,50$ & $-1,5$ & $-1,5$ & $-1,5$ & $-1,50$ & & \\
\hline & b & 18,1 & 18,2 & 17,9 & 18,07 & 18,2 & 18,1 & 18 & 18,10 & 17,5 & 17,7 & 17,9 & 17,70 & 18 & 18,1 & 18,1 & 18,07 & & \\
\hline \multirow[t]{3}{*}{3} & $\mathbf{L}$ & 92 & 91,7 & 92,3 & 92,00 & 92,4 & 92,5 & 91,7 & 92,20 & 89,3 & 89,7 & 89,8 & 89,60 & 89,4 & 89,4 & 90,2 & 89,67 & \multirow{3}{*}{2,69} & \multirow{3}{*}{$-0,17$} \\
\hline & a & $-0,5$ & $-0,5$ & $-0,5$ & $-0,50$ & $-0,5$ & $-0,5$ & $-0,5$ & $-0,50$ & $-1,1$ & $-1,1$ & $-1,2$ & $-1,13$ & $-1,1$ & $-1,1$ & $-1,2$ & $-1,13$ & & \\
\hline & b & 19,2 & 19,4 & 19,5 & 19,37 & 19,6 & 19,5 & 19,4 & 19,50 & 18,2 & 18,4 & 18,4 & 18,33 & 18,4 & 18,4 & 18,3 & 18,37 & & \\
\hline \multirow[t]{3}{*}{4} & L & 91,4 & 92 & 91,1 & 91,50 & 91,3 & 91,2 & 91,8 & 91,43 & 91,6 & 91,8 & 91,5 & 91,63 & 91,9 & 92,1 & 92 & 92,00 & \multirow{3}{*}{0,35} & \multirow{3}{*}{0,18} \\
\hline & $a$ & -11 & $-1,1$ & $-1,2$ & $-1,13$ & $-1,2$ & $-1,2$ & $-1,2$ & $-1,20$ & $-1,4$ & $-1,4$ & $-1,5$ & $\begin{array}{l}-1,43 \\
\end{array}$ & $-1,4$ & $-1,5$ & $-1,5$ & $-1,47$ & & \\
\hline & b & 17,7 & 18 & 17,7 & 17,80 & 17,6 & 17,6 & 17,7 & 17,63 & 17,9 & 18 & 17,9 & 17,93 & 17,9 & 17,8 & 18,1 & 17,93 & & \\
\hline \multirow[t]{3}{*}{5} & $\mathbf{L}$ & 91,4 & 91,5 & 91,6 & 91,50 & 92,7 & 91,9 & 92,4 & 92,33 & 89,5 & 89,3 & 89,4 & 89,40 & 89,4 & 89,5 & 89,5 & 89,47 & \multirow{3}{*}{2,54} & \multirow{3}{*}{$-0,55$} \\
\hline & $a$ & $-0,7$ & $-0,7$ & $-0,7$ & $-0,70$ & $-0,8$ & $-0,8$ & $-0,8$ & $-0,80$ & $\begin{array}{l}-1,3 \\
\end{array}$ & $-1,3$ & $-1,3$ & $-1,30$ & $-1,4$ & $-1,6$ & $-1,6$ & $-1,53$ & & \\
\hline & b & 19,7 & 19,7 & 19,9 & 19,77 & 19,8 & 19,7 & 20 & 19,83 & 18,5 & 18,3 & 18,6 & 18,47 & 18,7 & 18,7 & 18,5 & 18,63 & & \\
\hline 6 & $\mathbf{L}$ & 93,8 & 93,9 & 93,7 & 93,80 & 93,5 & 93,7 & 94,2 & 93,80 & 91,7 & 92,4 & 92,4 & 92,17 & 92,3 & 92,4 & 92,6 & 92,43 & & \\
\hline & $\mathbf{a}$ & $-0,8$ & $-0,8$ & $-0,7$ & $-0,77$ & $-0,8$ & $-0,7$ & $-0,8$ & $-0,77$ & $-0,9$ & $-0,9$ & $-0,9$ & $-0,90$ & $-0,9$ & $-0,9$ & $-0,9$ & $-0,90$ & 1,66 & 0,20 \\
\hline & b & 21 & 21,1 & 21,2 & 21,10 & 21,2 & 21,2 & 21,2 & 21,20 & 20,6 & 21 & 20,9 & 20,83 & 21 & 20,9 & 21 & 20,97 & & \\
\hline 7 & $\mathbf{L}$ & 94 & 94 & 93,5 & 93,83 & 93,4 & 94 & 93,6 & 93,67 & 92,4 & 92,4 & 92,8 & 92,53 & 92,5 & 92,6 & 92,3 & 92,47 & & \\
\hline & $a$ & -1 & -1 & $-0,9$ & $-0,97$ & $-0,9$ & -1 & -1 & $-0,97$ & $-1,2$ & $-1,2$ & $-1,2$ & $-1,20$ & $-1,3$ & $-1,2$ & $-1,2$ & $-1,23$ & 1,36 & $-0,08$ \\
\hline & b & 18,7 & 18,6 & 18,9 & 18,73 & 18,4 & 18,7 & $\begin{array}{l}18,6 \\
\end{array}$ & 18,57 & 18,5 & 18,3 & 18,4 & 18,40 & 18,1 & 18,2 & 18,5 & 18,27 & & \\
\hline 8 & $\mathbf{L}$ & 92,1 & 92,4 & 92 & 92,17 & 92,5 & 92,6 & 92,2 & 92,43 & 92,6 & 82,5 & 83,2 & 92,77 & 92,6 & 93,3 & 92,7 & 92,87 & & \\
\hline & $a$ & $-0,6$ & $-0,7$ & $-0,6$ & $-0,63$ & $-0,7$ & $-0,6$ & $-0,7$ & $-0,67$ & $-0,7$ & $-0,7$ & $-0,7$ & $-0,70$ & $-0,7$ & $-0,7$ & $-0,7$ & $-0,70$ & 0,87 & $-0,13$ \\
\hline & b & 20,2 & 19,9 & 19,9 & 20,00 & 20 & 19,9 & 20,1 & 20,00 & 20,4 & 20,6 & 20,9 & 20,63 & 20,6 & 20,8 & 20,8 & 20,73 & & \\
\hline 9 & $\mathbf{L}$ & 93,4 & 92,9 & 92,8 & 93,03 & 93,4 & 93 & 93,3 & 93,23 & 91 & 91,2 & 91,1 & 91,10 & 91,2 & 91,2 & 90,9 & 91,1 & & \\
\hline & $a$ & 91,6 & 91,2 & 91,8 & 91,53 & 92,2 & 92,3 & 92 & 92,17 & 92 & 92,1 & 91,9 & 92,00 & 92,2 & 92,1 & 92,5 & 92,27 & 2,16 & $-0,23$ \\
\hline & L & -1 & $-0,9$ & -1 & $-0,97$ & -1 & -1 & -1 & $-1,00$ & $-1,3$ & $-1,3$ & $-1,3$ & $-1,30$ & $-1,3$ & $-1,3$ & $-1,3$ & $-1,30$ & & \\
\hline & $a$ & 18,6 & 18,6 & 18,5 & 18,57 & 18.5 & 18.6 & 18.6 & 18.57 & 19,5 & 18,9 & 19,1 & 19,17 & 19 & 19 & 19 & 19,00 & & \\
\hline 10 & b & 93,3 & 93,5 & 94 & 93,6 & 93,6 & 93,7 & 93,7 & 93,67 & 92,6 & 92,9 & 93,2 & 92,90 & 93,3 & 93,3 & 93,5 & 93,37 & 2,36 & $-0,10$ \\
\hline & L & $-1,1$ & $-1,2$ & $-1,2$ & $-1,17$ & $-1,1$ & $-1,1$ & $-1,1$ & $-1,10$ & $-1,5$ & $-1,5$ & $-1,5$ & $-1,50$ & $-1,5$ & $-1,5$ & $-1,5$ & $-1,50$ & & \\
\hline
\end{tabular}


Quadro 14- Leituras de cor das amostras de E-max Press na espessura de 0,5mm cimentados com cimento autoadesivo.

\begin{tabular}{|c|c|c|c|c|c|c|c|c|c|c|c|c|c|c|c|c|c|c|c|}
\hline \multicolumn{20}{|c|}{ E-max Press 0,5mm Rely-X U200 } \\
\hline \multirow{2}{*}{$\begin{array}{l}\text { Corpo de } \\
\text { Prova }\end{array}$} & \multirow{2}{*}{ EIXO } & \multicolumn{4}{|c|}{ LEITURA INICIA Branco } & \multicolumn{4}{|c|}{ LEITURA INICIAL Preto } & \multicolumn{4}{|c|}{ LEITURA Final Branco } & \multicolumn{4}{|c|}{ LEITURA FINAL Preto } & \multicolumn{2}{|c|}{$\Delta$} \\
\hline & & LI1 & $\mathrm{LI} 2$ & LI3 & MEDIA LI & LF1 & LF2 & LF3 & MEDIA LF & Ll1 & $\mathrm{Ll} 2$ & LI3 & MEDIA LI & LF1 & LF2 & LF3 & MEDIA LF & $\Delta \mathrm{E}$ & $\Delta \mathrm{T}$ \\
\hline \multirow[t]{3}{*}{1} & $\mathbf{L}$ & 97,3 & 97,4 & 97,6 & 97,43 & 97,1 & 97,2 & 97,2 & 97,17 & 90,1 & 90,2 & 90 & 90,10 & 90,1 & 90,2 & 90,3 & 90,20 & \multirow{3}{*}{7,73} & \multirow{3}{*}{$-0,10$} \\
\hline & $\mathbf{a}$ & 0,9 & 0,9 & 0,9 & 0,90 & 1 & 1 & 1 & 1,00 & 0 & $-0,1$ & $-0,1$ & $-0,07$ & $-0,2$ & 0 & $-0,1$ & $-0,10$ & & \\
\hline & b & 28,1 & 28,2 & 28,2 & 28,17 & 28,3 & 28,3 & 28,2 & 28,27 & 26 & 26 & 25,8 & 25,93 & 25,8 & 26 & 25,5 & 25,77 & & \\
\hline \multirow[t]{3}{*}{2} & $\mathbf{L}$ & 91,5 & 90,8 & 91,5 & 91,27 & 90,9 & 90,6 & 91,1 & 90,87 & 93,2 & 93,3 & 93,4 & 93,30 & 93,3 & 93,3 & 93,2 & 93,27 & \multirow{3}{*}{2,72} & \multirow{3}{*}{$-0,20$} \\
\hline & $a$ & 0,4 & 0,5 & 0,6 & 0,50 & 0,7 & 0,3 & 0,6 & 0,53 & 0,3 & 0,4 & 0,2 & 0,30 & 0,2 & 0,3 & 0,4 & 0,30 & & \\
\hline & b & 22,4 & 22,8 & 22 & 22,40 & 22,1 & 22,9 & 22,4 & 22,47 & 24,2 & 24,6 & 23,8 & 24,20 & 23,7 & 24,1 & 24,2 & 24 & & \\
\hline \multirow[t]{3}{*}{3} & $\mathbf{L}$ & 91,4 & 91,6 & 91,5 & 91,50 & 91,5 & 91,6 & 91,5 & 91,53 & 90,4 & 90,9 & 90,7 & 90,67 & 90,5 & 90,8 & 90,8 & 90,70 & \multirow{3}{*}{1,13} & \multirow{3}{*}{0,04} \\
\hline & $a$ & 1 & 1 & 1 & 1,00 & 0,9 & 0,9 & 0,9 & 0,90 & 0,7 & 0,5 & 0,5 & 0,57 & 0,5 & 0,5 & 0,5 & 0,50 & & \\
\hline & b & 26,6 & 26,5 & 26,7 & 26,60 & 26,5 & 26,5 & 26,7 & 26,57 & 27,2 & 27,4 & 27,1 & 27,23 & 27,3 & 27,5 & 27,3 & 27,37 & & \\
\hline \multirow[t]{3}{*}{4} & L & 88,8 & 89 & 88,9 & 88,90 & 89,2 & 89,1 & 88,6 & 88,97 & 88,8 & 89,4 & 89,2 & 89,13 & 89,9 & 89 & 89 & 89,30 & \multirow{3}{*}{1,44} & \multirow{3}{*}{$-0,38$} \\
\hline & $\mathbf{a}$ & -1 & -1 & $-0,9$ & $-0,97$ & $-0,8$ & $-0,8$ & $-0,9$ & $\begin{array}{l}-0,83 \\
\end{array}$ & $\begin{array}{l}-1,3 \\
\end{array}$ & $\begin{array}{ll}-1,1 \\
\end{array}$ & $-1,2$ & $-1,20$ & $\begin{array}{l}-1,3 \\
\end{array}$ & $-1,3$ & $-1,3$ & $-1,30$ & & \\
\hline & b & 16,6 & 162 & 16,8 & 16,53 & 17 & 17,2 & 17,1 & 17,10 & 17,4 & 18,2 & 18,2 & 17,93 & 18,2 & 17,8 & 17,6 & 17,87 & & \\
\hline \multirow[t]{3}{*}{5} & $\mathbf{L}$ & 89,5 & 89,8 & 89 & 8943 & 89,3 & 89,1 & 88,8 & 89,07 & 91 & 90,8 & 91,2 & 91,00 & 90 & 89,8 & 90,3 & 90,03 & \multirow{3}{*}{2,39} & \multirow{3}{*}{0,50} \\
\hline & $a$ & 0 & 0,1 & 0 & 0,03 & 0 & 0 & 0,1 & 0,03 & $-0,1$ & 0 & 0 & $-0,03$ & 0 & 0,1 & 0 & 0,03 & & \\
\hline & b & 21,4 & 21,6 & 21,3 & 21,43 & 21,2 & 21,2 & 21 & 21,13 & 23,3 & 23,1 & 23,3 & 23,23 & 23,2 & 23,3 & 23,3 & 23,27 & & \\
\hline 6 & $L$ & 95,2 & 95,2 & 95,2 & 95,20 & 94,9 & 94,7 & 95,4 & 95,00 & 95,2 & 95,2 & 95,2 & 95,20 & 96,5 & 96,6 & 96,7 & 96,60 & & \\
\hline & $a$ & $-0,2$ & 0,2 & $-0,2$ & $-0,07$ & $-0,1$ & $-0,1$ & $-0,2$ & $-0,13$ & $-0,2$ & 0,2 & $-0,2$ & $-0,07$ & $-0,9$ & $-0,9$ & $-0,9$ & $-0,90$ & 2,02 & $-0,04$ \\
\hline & b & 18,7 & 18,6 & 18,9 & 18,73 & 18,9 & 18,9 & 18,5 & 18,77 & 18,7 & 18,6 & 189 & 18,73 & 20 & 20,2 & 20,2 & 20,13 & & \\
\hline 7 & $\mathbf{L}$ & 96 & 95,8 & 95,8 & 95,87 & 95,7 & 95,7 & 95,8 & 95,73 & 96 & 95,8 & 95,8 & 95,87 & 98,5 & 98,7 & 98,9 & 98,70 & & \\
\hline & $a$ & $-0,3$ & $-0,3$ & $-0,3$ & $-0,30$ & $-0,2$ & $-0,2$ & $-0,3$ & $-0,23$ & $-0,3$ & $-0,3$ & $-0,3$ & $-0,30$ & $\begin{array}{l}-0,3 \\
\end{array}$ & $-0,4$ & $-0,4$ & $\begin{array}{l}-0,37 \\
\end{array}$ & 4,53 & 0,19 \\
\hline & b & 21,8 & 21,9 & 21,7 & 21,80 & 21,7 & 22,1 & 21,9 & 21,90 & 21,8 & 21,9 & 21,7 & 21,80 & 25,1 & 24,9 & 24,9 & 24,97 & & \\
\hline 8 & $\mathbf{L}$ & 93,7 & 93,8 & 94,1 & 93,87 & 94,3 & 94 & 94 & 94,10 & 93,7 & 93,8 & 94,1 & 93,87 & 95,6 & 95,6 & 95,7 & 95,63 & & \\
\hline & $a$ & $-0,2$ & $-0,2$ & $-0,2$ & $-0,20$ & $-0,3$ & $-0,3$ & $-0,3$ & $-0,30$ & $\begin{array}{l}-0,2 \\
\end{array}$ & $-0,2$ & $-0,2$ & $-0,20$ & $-0,6$ & $-0,6$ & $-0,6$ & $-0,60$ & 2,48 & 0 \\
\hline & b & 23,2 & 23,6 & 23,5 & 23,43 & 23,5 & 23,1 & 23,7 & 23,43 & 23,2 & 23,6 & 23,5 & 23,43 & 25,4 & 25,3 & 25,3 & 25,33 & & \\
\hline 9 & $\mathbf{L}$ & 92,3 & 92,2 & 92,4 & 92,3 & 92,3 & 92,3 & 92,6 & 92,40 & 92,3 & 92,2 & 92,4 & 92,30 & 89,1 & 89,1 & 89,2 & 89,13 & & \\
\hline & $a$ & $-0,6$ & $-0,6$ & $-0,7$ & $-0,63$ & $-0,7$ & $-0,7$ & $-0,7$ & $-0,70$ & $-0,6$ & $-0,6$ & $-0,7$ & $-0,63$ & $-0,3$ & $-0,3$ & $-0,3$ & $-0,30$ & 3,71 & 0,67 \\
\hline & b & 20 & 20 & 19,8 & 19,93 & 20 & 19,8 & 19,6 & 19,80 & 20 & 20 & 19,8 & 19,93 & 23,1 & 23,1 & 232 & 23,13 & & \\
\hline & $\mathbf{L}$ & 93 & 92,9 & 92,8 & 92,90 & 92,9 & 93 & 93,1 & 93,00 & 93 & 92,9 & 92,8 & 92,90 & 91,9 & 91,9 & 91,5 & 91,77 & & \\
\hline 10 & $a$ & $-0,3$ & $-0,3$ & $-0,3$ & $-0,30$ & $-0,4$ & $-0,3$ & $-0,3$ & \begin{tabular}{|c|}
$-0,33$ \\
\end{tabular} & $-0,3$ & $-0,3$ & $-0,3$ & $-0,30$ & $-0,7$ & $-0,7$ & $-0,6$ & $-0,67$ & 1,16 & 0,28 \\
\hline & b & 21,3 & 21,2 & 21,4 & 21,30 & 21,3 & 21,7 & 21,7 & 21,57 & 21,3 & 21,2 & 21,4 & 21,30 & 21,9 & 22 & 22,5 & 22,13 & & \\
\hline
\end{tabular}


Quadro 15- Leituras de cor das amostras de E-max Press na espessura de 1,0mm cimentados com cimento autoadesivo.

\begin{tabular}{|c|c|c|c|c|c|c|c|c|c|c|c|c|c|c|c|c|c|c|c|}
\hline \multicolumn{20}{|c|}{ E-max Press 1,0mmRely-X U200 } \\
\hline \multirow{2}{*}{$\begin{array}{l}\text { Corpo de } \\
\text { Prova }\end{array}$} & \multirow[b]{2}{*}{ EIXO } & \multicolumn{4}{|c|}{ LEITURA INICIA Branco } & \multicolumn{4}{|c|}{ LEITURA INICIAL Preto } & \multicolumn{4}{|c|}{ LEITURA Final Branco } & \multicolumn{4}{|c|}{ LEITURA FINAL Preto } & \multicolumn{2}{|c|}{$\Delta$} \\
\hline & & LI1 & $\mathrm{LI} 2$ & LI3 & MEDIA LI & LF1 & LF2 & LF3 & MEDIA LF & LI1 & $\mathrm{LI} 2$ & LI3 & MEDIA LI & LF1 & LF2 & LF3 & MEDIA LF & $\Delta \mathrm{E}$ & $\Delta \mathrm{T}$ \\
\hline \multirow[t]{3}{*}{1} & $\mathbf{L}$ & 93,1 & 93,2 & 24,5 & 23,60 & 94,8 & 94,8 & 93,9 & 94,50 & 96,1 & 97 & 96,8 & 96,63 & 95,1 & 95,2 & 95,2 & 95,17 & \multirow{3}{*}{3,09} & \multirow{3}{*}{0,61} \\
\hline & a & $-0,8$ & $-0,8$ & $-0,9$ & $-0,83$ & $-0,9$ & $-0,9$ & $-0,9$ & $-0,90$ & -1 & $-1,2$ & $-1,2$ & $-1,13$ & -1 & -1 & -1 & $-1,00$ & & \\
\hline & b & 19 & 19 & 18,7 & 18,90 & 18,8 & 19 & 19,3 & 19,03 & 19,3 & 19,3 & 19,6 & 19,40 & 19,9 & 19,7 & 19,7 & 19,77 & & \\
\hline \multirow[t]{3}{*}{2} & L & 93,3 & 93,7 & 93,4 & 93,47 & 93,8 & 94,2 & 93,9 & 93,97 & 92,8 & 92,8 & 92,7 & 92,77 & 92,8 & 92,8 & 92,9 & 92,83 & \multirow{3}{*}{0,77} & \multirow{3}{*}{$-0,39$} \\
\hline & $\mathbf{a}$ & $-0,8$ & $-0,8$ & $-0,8$ & $-0,80$ & $-0,8$ & $-0,8$ & $-0,8$ & $-0,80$ & $-0,9$ & -1 & -1 & $-0,97$ & -1 & -1 & -1 & $-1,00$ & & \\
\hline & b & 19,2 & 19,3 & 19,7 & 19,40 & 19,5 & 19,7 & 19,6 & 19,60 & 19,3 & 19 & 19,1 & 19,13 & 19,2 & 19,2 & 19,4 & 19,27 & & \\
\hline \multirow[t]{3}{*}{3} & $L$ & 90,4 & 90,3 & 89,9 & 90,20 & 90 & 90,2 & 90,2 & 90,13 & 89,5 & 90 & 89,6 & 89,70 & 90,9 & 90,3 & 90,4 & 90,53 & \multirow{3}{*}{0,08} & \multirow{3}{*}{0,76} \\
\hline & $a$ & $-0,2$ & $-0,1$ & $-0,1$ & $-0,13$ & $-0,1$ & $-0,1$ & $-0,1$ & $-0,10$ & $-0,2$ & $-0,3$ & $-0,3$ & $-0,27$ & $-0,3$ & $-0,3$ & $-0,3$ & $-0,30$ & & \\
\hline & b & 20,6 & 20,7 & 21,3 & 20,87 & 20,9 & 20,9 & 20,9 & 20,90 & 20,9 & 20,6 & 20,7 & 20,73 & 20,6 & 20,5 & 20,7 & 20,60 & & \\
\hline \multirow[t]{3}{*}{4} & L & 94,1 & 94 & 94 & 94,03 & 94,3 & 94,2 & 948 & 94,43 & 93,5 & 93,2 & 93,8 & 93,50 & 93,1 & 93,9 & 93 & 93,33 & \multirow{3}{*}{0,74} & \multirow{3}{*}{$-0,18$} \\
\hline & $a$ & $\begin{array}{ll}-1,2 \\
\end{array}$ & $-1,1$ & $-1,1$ & $-1,13$ & $-1,2$ & $-1,2$ & $-1,3$ & $\begin{array}{l}-1,23 \\
\end{array}$ & $-1,4$ & $\begin{array}{l}-1,3 \\
\end{array}$ & $-1,3$ & $\begin{array}{l}-1,33 \\
\end{array}$ & $\begin{array}{l}-1,3 \\
\end{array}$ & $-1,3$ & $-1,3$ & $-1,30$ & & \\
\hline & b & 17,8 & 17,9 & 17,9 & 17,87 & 18 & 18 & 17,8 & 17,93 & 17,2 & 17,5 & 17,5 & 17,40 & 17,3 & 17,8 & 17,6 & 17,57 & & \\
\hline \multirow[t]{3}{*}{5} & $\mathbf{L}$ & 92,5 & 92,5 & 92,4 & 92,47 & 92,4 & 92,6 & 92,5 & 92,50 & 94,1 & 94 & 94,7 & 94,27 & 94 & 94,7 & 94,7 & 94,47 & \multirow{3}{*}{2,29} & \multirow{3}{*}{0,16} \\
\hline & $a$ & $-0,8$ & $-0,8$ & $-0,8$ & $-0,80$ & $-0,8$ & $-0,8$ & $-0,8$ & $-0,80$ & $-0,6$ & $-0,6$ & $-0,7$ & $-0,63$ & $-0,6$ & $-0,7$ & $-0,7$ & $-0,67$ & & \\
\hline & b & 20,1 & 20,3 & 20,2 & 20,20 & 20,2 & 20,2 & 20,1 & 20,17 & 21,5 & 21,8 & 21,5 & 21,60 & 21,7 & 21,6 & 21,6 & 21,63 & & \\
\hline 6 & $\mathbf{L}$ & 93,2 & 94,2 & 94,1 & 93,83 & 93,3 & 93,8 & 94,4 & 93,83 & 92,6 & 93,5 & 92,6 & 92,60 & 93,7 & 91,7 & 93,6 & 93,00 & & \\
\hline & a & -1 & $-0,9$ & -1 & $-0,97$ & -1 & $-0,9$ & $-1,1$ & $-1,00$ & $-0,9$ & $-0,9$ & -1 & $-0,93$ & -1 & $-0,8$ & $-0,9$ & $-0,90$ & 0,96 & 0,06 \\
\hline & b & 18,3 & 18,2 & 18,2 & 18,23 & 18,1 & 18,3 & 18,4 & 18,27 & 18,4 & 18,6 & 18,4 & 18,47 & 18,6 & 18,4 & 18,5 & 18,50 & & \\
\hline 7 & $\mathbf{L}$ & 93 & 93,1 & 93 & 93,03 & 93,4 & 92,8 & 93,2 & 93,13 & 92,9 & 92,8 & 93,1 & 92,93 & 92,9 & 93 & 93,5 & 93,13 & & \\
\hline & a & $-1,5$ & $-1,5$ & $-1,5$ & $-1,50$ & $-1,5$ & $-1,5$ & $-1,5$ & $-1,50$ & $-1,6$ & $-1,5$ & $-1,5$ & $-1,53$ & $-1,5$ & $-1,5$ & $-1,5$ & $-1,50$ & 0,20 & 0,14 \\
\hline & b & 18 & 18,1 & 18,1 & 18,07 & 18,1 & 18 & 17,8 & 17,97 & 17,9 & 17,9 & 17,9 & 17,90 & 18,1 & 18,1 & 18,1 & 18,10 & & \\
\hline 8 & $\mathbf{L}$ & 93,5 & 93,7 & 93,9 & 93,70 & 93,9 & 94,1 & 94,4 & 94,13 & 93,4 & 93,6 & 93,6 & 93,53 & 93,6 & 93,6 & 93,4 & 93,53 & & \\
\hline & $a$ & $-0,9$ & $-0,8$ & $-0,8$ & $-0,83$ & $-0,8$ & $-0,8$ & $-0,8$ & $-0,80$ & $-0,8$ & $-0,8$ & $-0,8$ & $-0,80$ & $-0,8$ & $-0,8$ & $-0,8$ & $-0,80$ & 0,20 & $-0,43$ \\
\hline & b & 19,7 & 20 & 20 & 19,90 & 20,2 & 20,2 & 20,2 & 20,20 & 19,8 & 19,7 & 19,9 & 19,80 & 20 & 19,7 & 20 & 19,90 & & \\
\hline 9 & $L$ & 92,6 & 92,9 & 92,4 & 92,63 & 91,1 & 91,4 & 91,2 & 91,23 & 92,1 & 93 & 92,2 & 92,43 & 93,5 & 92,2 & 95,9 & 93,87 & & \\
\hline & $\mathbf{a}$ & -1 & $-1,1$ & -1 & $-1,03$ & $-0,9$ & $-0,9$ & $-0,9$ & $-0,90$ & $-0,5$ & $-0,7$ & $-0,5$ & $-0,57$ & $-0,7$ & $-0,5$ & -1 & $-0,73$ & 0,76 & 0,10 \\
\hline & b & 20,2 & 19,7 & 19,9 & 19,93 & 19,9 & 19,9 & 19,8 & 19,87 & 21 & 20,1 & 20,4 & 20,50 & 20,2 & 20,3 & 19,7 & 20,07 & & \\
\hline & L & 97,8 & 97,8 & 97,8 & 97,80 & 97,8 & 98,9 & 99 & 98,57 & 95 & 94,9 & 95 & 94,97 & 95 & 95,1 & 95,5 & 95,20 & & \\
\hline 10 & $\mathbf{L}$ & 93,1 & 93,2 & 24,5 & 23,60 & 94,8 & 94,8 & 93,9 & 94,50 & 96,1 & 97 & 96,8 & 96,63 & 95,1 & 95,2 & 95,2 & 95,17 & 0,80 & $-0,54$ \\
\hline & $a$ & $-0,8$ & $-0,8$ & $-0,9$ & $-0,83$ & $-0,9$ & $-0,9$ & $-0,9$ & $-0,90$ & -1 & $-1,2$ & $-1,2$ & $-1,13$ & -1 & -1 & -1 & $-1,00$ & & \\
\hline
\end{tabular}


Quadro 16- Leituras de cor das amostras de ZirPress na espessura de 0,5mm cimentados com cimento fotoativado.

\begin{tabular}{|c|c|c|c|c|c|c|c|c|c|c|c|c|c|c|c|c|c|c|c|}
\hline \multicolumn{20}{|c|}{ ZirPress 0,5mm Variolink II } \\
\hline \multirow{2}{*}{$\begin{array}{l}\text { Corpo de } \\
\text { Prova }\end{array}$} & \multirow[b]{2}{*}{ EIXO } & \multicolumn{4}{|c|}{ LEITURA INICIA Branco } & \multicolumn{4}{|c|}{ LEITURA INICIAL Preto } & \multicolumn{4}{|c|}{ LEITURA Final Branco } & \multicolumn{4}{|c|}{ LEITURA FINAL Preto } & \multicolumn{2}{|c|}{$\Delta$} \\
\hline & & LI1 & $\mathrm{LI} 2$ & LI3 & MEDIA LI & LF1 & LF2 & LF3 & MEDIA LF & LI1 & $\mathrm{LI} 2$ & LI3 & MEDIA LI & LF1 & LF2 & LF3 & MEDIA LF & $\Delta \mathrm{E}$ & $\Delta \mathrm{T}$ \\
\hline \multirow{3}{*}{1} & $\mathbf{L}$ & 90,1 & 89,9 & 90,6 & 90,17 & 90,4 & 89,8 & 90,6 & 90,27 & 92,1 & 91,5 & 91,3 & 91,63 & 91,5 & 91,5 & 91,5 & 91,50 & \multirow{3}{*}{1,76} & \multirow{3}{*}{0,40} \\
\hline & $\mathbf{a}$ & 0,4 & 0,5 & 0,3 & 0,40 & 0,2 & 0,3 & 0,2 & 0,23 & $-0,4$ & $-0,3$ & $-0,2$ & $-0,30$ & 0,1 & 0 & 0,1 & 0,07 & & \\
\hline & b & 23,1 & 23,7 & 23,8 & 23,53 & 23,7 & 23,8 & 23,5 & 23,67 & 23,9 & 24,3 & 24,4 & 24,20 & 24,8 & 24,8 & 24,5 & 24,7 & & \\
\hline \multirow{3}{*}{2} & $\mathbf{L}$ & 92,5 & 92,4 & 92,6 & 92,50 & 92,3 & 92,8 & 92,5 & 92,53 & 93,8 & 94,3 & 94,5 & 94,20 & 94,4 & 93,5 & 93,2 & 93,2 & \multirow{3}{*}{2,39} & \multirow{3}{*}{$-0,01$} \\
\hline & $\mathbf{a}$ & 0,8 & $-0,8$ & $-0,8$ & $-0,27$ & $-0,8$ & $-0,8$ & $-0,8$ & $-0,80$ & $-0,8$ & $-0,8$ & -1 & $-0,87$ & -1 & $-0,8$ & $-0,6$ & $-0,80$ & & \\
\hline & b & 23,5 & 23,9 & 23,9 & 23,77 & 24,1 & 23,6 & 23,4 & 23,70 & 25,5 & 25,4 & 25,1 & 25,33 & 25,5 & 25,3 & 25 & 25,17 & & \\
\hline \multirow{3}{*}{3} & $L$ & 94,6 & 94,5 & 94,5 & 94,53 & 94,6 & 94,7 & 94,8 & 94,70 & 93,2 & 93,3 & 93,1 & 93,20 & 93,1 & 93,3 & 93,2 & 93,20 & \multirow{3}{*}{1,38} & \multirow{3}{*}{0,48} \\
\hline & $a$ & 0,6 & 0,6 & 0,6 & 0,60 & 0,5 & 0,5 & 0,5 & 0,50 & 1 & 0,9 & 0,8 & 0,90 & 0,7 & 0,8 & 0,7 & 0,73 & & \\
\hline & b & 28,6 & 28,7 & 28,8 & 28,70 & 28,6 & 28,7 & 28,6 & 28,63 & 28,9 & 28,9 & 28,8 & 28,87 & 28,2 & 28,4 & 28 & 28,2 & & \\
\hline \multirow{3}{*}{4} & L & 91,6 & 91,4 & 91,3 & 91,43 & 91,2 & 91,5 & 91,6 & 91,43 & & & & & & & & & & \\
\hline & $a$ & 1,2 & $-1,1$ & $-1,1$ & $-0,33$ & $-1,1$ & $-1,1$ & -1 & $-1,07$ & & & & & & & & & & \\
\hline & b & 20,1 & 20,5 & 20,3 & 20,30 & 20,7 & 21,2 & 21,3 & 21,07 & & & & & & & & & & \\
\hline \multirow{3}{*}{5} & $\mathbf{L}$ & 90,2 & 90,1 & 90,2 & 90,17 & 90,3 & 90,1 & 90,2 & 90,20 & 90,7 & 90,9 & 90,8 & 90,80 & 90,7 & 90,9 & 90,6 & 90,73 & \multirow{3}{*}{0,70} & \multirow{3}{*}{0,27} \\
\hline & $a$ & 0 & 0 & $-0,1$ & $-0,03$ & 0 & 0 & $-0,1$ & $-0,03$ & $-0,2$ & $-0,3$ & $-0,3$ & $-0,27$ & $-0,3$ & $-0,3$ & $-0,2$ & $-0,27$ & & \\
\hline & b & 24,1 & 23,5 & 23,4 & 23,67 & 23,6 & 23,6 & 23,5 & 23,57 & 24,1 & 24 & 23,5 & 23,87 & 23,4 & 23,5 & 23,6 & 23,50 & & \\
\hline \multirow{3}{*}{6} & $\mathbf{L}$ & 93,2 & 92,9 & 92,9 & 93,00 & 93 & 93,1 & 93,2 & 93,10 & 92,1 & 92,1 & 92,2 & 92,13 & 92,2 & 92,3 & 92,3 & 92,27 & \multirow{3}{*}{1} & \\
\hline & a & 0,8 & 0,9 & 0,9 & 0,87 & 0,9 & 0,8 & 0,8 & 0,83 & 0,4 & 0,4 & 0,3 & 0,37 & 0,4 & 0,4 & 0,3 & 0,37 & & 0,13 \\
\hline & b & 27,6 & 28,2 & 28,3 & 28,03 & 28,6 & 28 & 27,9 & 28,17 & 28,2 & 28 & 27,9 & 28,03 & 28,3 & 28,5 & 28,1 & 28,30 & & \\
\hline & $\mathbf{L}$ & 91,7 & 91,8 & 91,6 & 91,70 & 91,8 & 91,8 & 91,4 & 91,67 & 92,3 & 92,1 & 92,6 & 92,23 & 92,4 & 91,7 & 91,8 & 91,97 & & \\
\hline 7 & a & 0,3 & 0,3 & 0,2 & 0,27 & 0,3 & 0,2 & 0,2 & 0,23 & 0,4 & 0,4 & 0,3 & 0,37 & 0,3 & 0,4 & 0,4 & 0,37 & 1,08 & $-0,77$ \\
\hline & b & 26,6 & 26,7 & 26,4 & 26,57 & 23,2 & 26,7 & 26,6 & 25,5 & 27,9 & 27,4 & 27,2 & 27,50 & 27,1 & 27,9 & 27,9 & 27,63 & & \\
\hline & $\mathbf{L}$ & 90,7 & 90,9 & 90,6 & 90,73 & 90,7 & 90,6 & 91 & 90,77 & & & & & & & & & & \\
\hline 8 & $a$ & 0,6 & 0,6 & 0,6 & 0,60 & 0,5 & 0,6 & 0,7 & 0,60 & & & & & & & & & & \\
\hline & b & 26 & 26 & 25,8 & 25,93 & 25,9 & 26,3 & 26,4 & 26,20 & & & & & & & & & & \\
\hline & L & 89,9 & 89,5 & 89,8 & 89,73 & 89,7 & 89,6 & 89,4 & 89,57 & 88,8 & 88,4 & 88,6 & 88,60 & 88,9 & 88,9 & 88,1 & 88,97 & & \\
\hline 9 & $\mathbf{a}$ & 0 & 0 & 0 & 0 & 0 & 0 & 0,0 & 0,03 & $-0,3$ & $-0,2$ & $-0,2$ & $-0,23$ & $\begin{array}{l}-0,3 \\
\end{array}$ & $\begin{array}{l}-0,3 \\
\end{array}$ & 0,3 & 0,10 & 1,60 & 0,06 \\
\hline & b & 25 & 25,5 & 25,2 & 25,23 & 25,5 & 25,4 & 25,7 & 25,53 & 26,2 & 26,3 & 26,5 & 26,33 & 26,4 & 26,5 & 26,4 & 26,43 & & \\
\hline & $\mathbf{L}$ & 87,9 & 88,1 & 87,9 & 87,97 & 87,9 & 88 & 87,8 & 87,90 & 88,3 & 88,3 & 88,1 & 88,23 & 88,1 & 88 & 88 & 88,03 & & \\
\hline 10 & $a$ & 0,7 & 0,6 & 0,7 & 0,67 & 0,7 & 0,8 & 0,7 & 0,73 & 0,3 & 0,3 & 0,3 & 0,30 & 0,3 & 0,3 & 0,4 & 0,33 & 0,86 & 0 \\
\hline & b & 27,1 & 26,7 & 27,2 & 27,00 & 27,1 & 27,5 & 27 & 27,20 & 27,8 & 27,6 & 27,8 & 27,73 & 27,6 & 27,8 & 28,1 & 27,83 & & \\
\hline
\end{tabular}


Quadro 17- Leituras de cor das amostras de ZirPress na espessura de 1,0mm cimentados com cimento fotoativado.

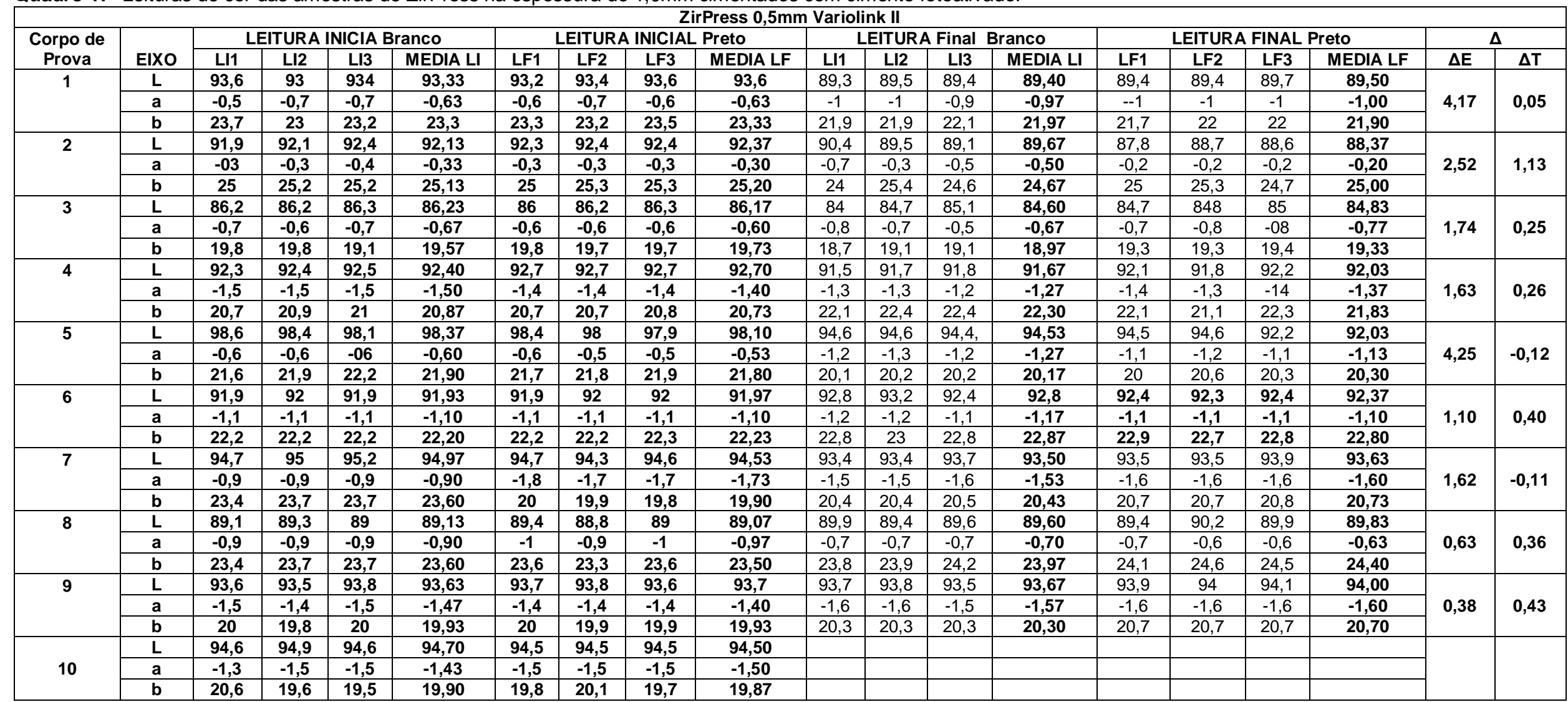


Quadro 18- Leituras de cor das amostras de ZirPress na espessura de 0,5mm cimentados com cimento autoadesivo.

\begin{tabular}{|c|c|c|c|c|c|c|c|c|c|c|c|c|c|c|c|c|c|c|c|}
\hline \multicolumn{20}{|c|}{ ZirPress 0,5mm Rely-X U200 } \\
\hline \multirow{2}{*}{$\begin{array}{l}\text { Corpo de } \\
\text { Prova }\end{array}$} & \multirow[b]{2}{*}{ EIXO } & \multicolumn{4}{|c|}{ LEITURA INICIA Branco } & \multicolumn{4}{|c|}{ LEITURA INICIAL Preto } & \multicolumn{4}{|c|}{ LEITURA Final Branco } & \multicolumn{4}{|c|}{ LEITURA FINAL Preto } & \multicolumn{2}{|c|}{$\Delta$} \\
\hline & & LI1 & $\mathrm{LI} 2$ & LI3 & MEDIA LI & LF1 & LF2 & LF3 & MEDIA LF & LI1 & LI2 & LI3 & MEDIA LI & LF1 & LF2 & LF3 & MEDIA LF & $\Delta \mathrm{E}$ & $\Delta \mathbf{T}$ \\
\hline \multirow[t]{3}{*}{1} & $\mathbf{L}$ & 91,3 & 90,5 & 91 & 90,93 & 91,1 & 90,9 & 91 & 91,00 & 90,5 & 91 & 91,5 & 91,00 & 91,6 & 91,5 & 91,4 & 91,50 & \multirow{3}{*}{0,55} & \multirow{3}{*}{0,50} \\
\hline & $\mathbf{a}$ & $-0,1$ & 0 & $-0,1$ & $-0,07$ & $-0,1$ & $-0,2$ & $-0,2$ & $-0,17$ & $-0,1$ & $-0,2$ & $-0,3$ & $-0,20$ & $-0,3$ & $-0,3$ & $-0,3$ & $-0,30$ & & \\
\hline & b & 26,6 & 26,7 & 26,6 & 26,63 & 26,8 & 27 & 26,7 & 26,83 & 26,9 & 27,3 & 27,3 & 27,17 & 27,8 & 27,7 & 27,6 & 27,70 & & \\
\hline \multirow[t]{3}{*}{2} & L & 91,7 & 91,6 & 91,6 & 91,63 & 92 & 92,1 & 91,4 & 91,83 & 91,2 & 91,5 & 92 & 91,57 & 92 & 92,4 & 91,9 & 92,10 & \multirow{3}{*}{1,01} & \multirow{3}{*}{0,41} \\
\hline & $\mathbf{a}$ & $-1,4$ & $-1,4$ & $-1,4$ & $-1,40$ & $\begin{array}{l}-1,3 \\
\end{array}$ & $-1,4$ & $-1,4$ & $-1,37$ & $\begin{array}{l}-1,3 \\
\end{array}$ & $-1,2$ & $-1,4$ & $-1,30$ & $-1,4$ & $-1,5$ & $-1,5$ & $-1,47$ & & \\
\hline & b & 22,5 & 22,5 & 22,6 & 22,53 & 22,5 & 22,7 & 22,7 & 22,63 & 23,2 & 23,8 & 23,6 & 23,53 & 23,9 & 23,8 & 23,8 & 23,83 & & \\
\hline \multirow[t]{3}{*}{3} & $L$ & 92,9 & 92,5 & 92,6 & 92,67 & 92,6 & 92,4 & 92,5 & 92,50 & 92,8 & 93,1 & 93,3 & 93,07 & 93,2 & 93,5 & 93,5 & 93,33 & \multirow{3}{*}{0,92} & \multirow{3}{*}{$-0,24$} \\
\hline & $a$ & $-0,9$ & $-0,8$ & $-0,9$ & $-0,87$ & $-0,9$ & $-0,9$ & -09 & $-0,90$ & $-0,8$ & $-0,9$ & $-0,9$ & $-0,87$ & $-0,9$ & -1 & $-0,9$ & $-0,93$ & & \\
\hline & b & 24 & 24,3 & 24,1 & 24,13 & 23,3 & 23,6 & 23,7 & 23,53 & 24,8 & 25,1 & 25 & 24,97 & 25,2 & 25,1 & 25,4 & 25,23 & & \\
\hline \multirow[t]{3}{*}{4} & L & 86 & 86 & 85,8 & 85,93 & 85,8 & 85,5 & 86 & 85,77 & 87,6 & 87,8 & 87,5 & 87,63 & 87,5 & 87,6 & 87,5 & 87,53 & \multirow{3}{*}{2,9} & \multirow{3}{*}{0,37} \\
\hline & $a$ & $\begin{array}{l}-0,8 \\
\end{array}$ & $-0,6$ & -07 & $-0,70$ & $-0,7$ & $-0,7$ & $-0,8$ & $\begin{array}{l}-0,73 \\
\end{array}$ & $-0,2$ & $-0,2$ & $-0,3$ & $-0,23$ & $-0,3$ & $\begin{array}{l}-0,3 \\
\end{array}$ & $-0,3$ & $-0,30$ & & \\
\hline & b & 25,4 & 25,5 & 25 & 25,30 & 25,5 & 25,3 & 25,2 & 25,33 & 27,8 & 27,9 & 27,1 & 27,6 & 27,4 & 27,3 & 26,5 & 27,07 & & \\
\hline \multirow[t]{3}{*}{5} & $\mathbf{L}$ & 88,2 & 88,5 & 87,2 & 87,97 & 87,7 & 87,8 & 87,6 & 87,70 & & & & & & & & & & \\
\hline & $a$ & $-1,9$ & $-1,9$ & $-1,8$ & $-1,87$ & $-1,8$ & $-1,8$ & $-1,9$ & $\begin{array}{l}-1,83 \\
\end{array}$ & & & & & & & & & & \\
\hline & b & 16,1 & 16,4 & 15,9 & 16,13 & 15,8 & 15,8 & 15,9 & 15,83 & & & & & & & & & & \\
\hline \multirow[t]{3}{*}{6} & $\mathbf{L}$ & 91,5 & 91,8 & 91,7 & 91,67 & 91,6 & 91,8 & 92 & 91,80 & & & & & & & & & & \\
\hline & $\mathbf{a}$ & $-0,5$ & $-0,5$ & $-0,4$ & $-0,47$ & $-0,4$ & $-0,5$ & $-0,6$ & $-0,50$ & & & & & & & & & & \\
\hline & b & 25,1 & 25,4 & 25,3 & 25,27 & 25 & 25,2 & 25,4 & 25,2 & & & & & & & & & & \\
\hline \multirow[t]{3}{*}{7} & $\mathbf{L}$ & 91 & 90,4 & 90,4 & 90,6 & 90,6 & 89,9 & 90,4 & 90,30 & & & & & & & & & & \\
\hline & $\mathbf{a}$ & $-1,6$ & $-1,8$ & $-1,8$ & $-1,73$ & $-1,9$ & $-1,8$ & $-1,8$ & $-1,83$ & & & & & & & & & & \\
\hline & b & 18 & 17,8 & 17,9 & 17,90 & 17,6 & 17,5 & 17,7 & 17,60 & & & & & & & & & & \\
\hline 8 & $\mathbf{L}$ & 93,8 & 93,6 & 93,5 & 93,63 & 93,5 & 93,8 & 93,8 & 93,70 & 93,2 & 93,2 & 93 & 93,13 & 92,5 & 92,9 & 92,5 & 92,63 & & \\
\hline & $\mathbf{a}$ & 0,5 & 0,5 & 0,6 & 0,53 & 0,6 & 0,5 & 0,5 & 0,53 & 0 & 0,1 & 0,1 & 0,07 & 0,1 & 0,2 & 0,1 & 0,13 & 3,93 & 0,66 \\
\hline & b & 30,5 & 30,2 & 30,5 & 30,40 & 30,5 & 30,2 & 30,3 & 30,33 & 26,4 & 26,5 & 26,7 & 26,53 & 27,3 & 27,3 & 26,7 & 27,10 & & \\
\hline 9 & $\mathbf{L}$ & 91,2 & 91,2 & 91,2 & 91,2 & 91,2 & 91,2 & 91 & 91,13 & & & & & & & & & & \\
\hline & $\mathbf{a}$ & -2 & $-1,9$ & $-1,8$ & $-1,90$ & $-1,8$ & -2 & $-1,9$ & $-1,90$ & & & & & & & & & & \\
\hline & b & 16,9 & 16,6 & 16,9 & 16,80 & 17 & 16,5 & 16,6 & 16,70 & & & & & & & & & & \\
\hline & $\mathbf{L}$ & 92,9 & 92,5 & 92,8 & 92,73 & 92,4 & 92,6 & 92,7 & 92,57 & 93,6 & 91,8 & 92,2 & 92,53 & 90 & 92,4 & 91,5 & 91,30 & & \\
\hline 10 & $a$ & $-0,9$ & $-0,7$ & $-0,8$ & $-0,80$ & $-0,7$ & $-0,8$ & $-0,6$ & $-0,70$ & $-0,4$ & $-0,6$ & $-0,5$ & $-0,50$ & $\begin{array}{l}-0,7 \\
\end{array}$ & $-0,5$ & $-0,5$ & $-0,57$ & 1,45 & 1,18 \\
\hline & b & 25,5 & 26,6 & 26,9 & 26,33 & 36,5 & 26,2 & 26,8 & 26,50 & 25,2 & 25,4 & 24,2 & 24,93 & 24 & 24,5 & 24,1 & 24,20 & & \\
\hline
\end{tabular}


Quadro 19- Leituras de cor das amostras de ZirPress na espessura de 1,0mm cimentados com cimento autoadesivo.

\begin{tabular}{|c|c|c|c|c|c|c|c|c|c|c|c|c|c|c|c|c|c|c|c|}
\hline \multicolumn{20}{|c|}{ ZirPress 1,0mm Rely-X U200 } \\
\hline \multirow{2}{*}{$\begin{array}{c}\text { Corpo de } \\
\text { Prova } \\
\end{array}$} & \multirow{2}{*}{ EIXO } & \multicolumn{4}{|c|}{ LEITURA INICIA Branco } & \multicolumn{4}{|c|}{ LEITURA INICIAL Preto } & \multicolumn{4}{|c|}{ LEITURA Final Branco } & \multicolumn{4}{|c|}{ LEITURA FINAL Preto } & \multicolumn{2}{|c|}{$\Delta$} \\
\hline & & Ll1 & $\mathrm{LI} 2$ & LI3 & MEDIA LI & LF1 & LF2 & LF3 & MEDIA LF & LI1 & LI2 & LI3 & MEDIA LI & LF1 & LF2 & LF3 & MEDIA LF & $\Delta \mathrm{E}$ & $\Delta \mathrm{T}$ \\
\hline \multirow[t]{3}{*}{1} & $\mathbf{L}$ & 91,6 & 92,2 & 92,7 & 92,17 & 91,3 & 92,1 & 92,8 & 92,07 & 93,7 & 94,2 & 94,1 & 94,00 & 94,1 & 94,3 & 94,4 & 94,27 & \multirow{3}{*}{2,38} & \multirow{3}{*}{$-0,02$} \\
\hline & $\mathbf{a}$ & $-1,1$ & $-1,1$ & $-1,2$ & $-1,13$ & $-0,9$ & $-1,1$ & $-1,2$ & $-1,07$ & $-1,7$ & $-1,7$ & $-1,7$ & $-1,70$ & $-1,7$ & $-1,7$ & $-1,8$ & $-1,73$ & & \\
\hline & b & 19,9 & 20,2 & 19,9 & 20,00 & 20,5 & 20,2 & 20,1 & 20,27 & 21,4 & 21,4 & 21,4 & 21,40 & 21,5 & 21,4 & 21,4 & 21,43 & & \\
\hline \multirow[t]{3}{*}{2} & $\mathbf{L}$ & 90,4 & 90,4 & 90,5 & 90,43 & 90,8 & 90,8 & 90,8 & 90,80 & 91,4 & 91,4 & 91,5 & 91,43 & 91,5 & 91,6 & 91,7 & 91,60 & \multirow{3}{*}{1,44} & \multirow{3}{*}{$-0,26$} \\
\hline & $a$ & $-1,7$ & $-1,6$ & $-1,6$ & $-1,63$ & $-1,7$ & $-1,6$ & $-1,7$ & $-1,67$ & $-2,2$ & $-2,2$ & -22 & $-2,20$ & $\begin{array}{l}-2,2 \\
\end{array}$ & $-2,2$ & $-2,2$ & $-2,20$ & & \\
\hline & b & 16,6 & 16,8 & 16,8 & 16,73 & 17 & 16,9 & 17 & 16,97 & 17,5 & 17,7 & 17,6 & 17,60 & 17,6 & 17,7 & 17,7 & 17,67 & & \\
\hline \multirow[t]{3}{*}{3} & $\mathbf{L}$ & 91 & 91,2 & 91,3 & 91,17 & 91,2 & 91,5 & 91,3 & 91,33 & 91,4 & 91,8 & 91,7 & 91,63 & 91,7 & 91,7 & 91,8 & 91,73 & \multirow{3}{*}{0,93} & \multirow{3}{*}{0,07} \\
\hline & $a$ & $-1,8$ & $-1,8$ & $-1,8$ & $-1,80$ & $-1,8$ & $-1,8$ & $-1,8$ & $-1,80$ & $\begin{array}{l}-2,3 \\
\end{array}$ & $-2,3$ & $-2,3$ & $-2,30$ & $\begin{array}{l}-2,3 \\
\end{array}$ & $-2,3$ & $\begin{array}{l}-2,3 \\
\end{array}$ & $-2,30$ & & \\
\hline & b & 18,1 & 18,3 & 18,3 & 18,23 & 18,5 & 18,1 & 18,3 & 18,30 & 18,7 & 18,9 & 19 & 18,87 & 19,1 & 19,1 & 19,1 & 19,10 & & \\
\hline \multirow[t]{3}{*}{4} & $\mathbf{L}$ & 88,2 & 88,1 & 88,1 & 88,13 & 88,3 & 88,6 & 88,6 & 88,50 & & & & & & & & & & \\
\hline & $\mathbf{a}$ & $-1,3$ & $-1,3$ & $-1,3$ & $-1,30$ & $-1,3$ & $\begin{array}{l}-1,6 \\
\end{array}$ & $\begin{array}{l}-1,3 \\
\end{array}$ & $-1,40$ & & & & & & & & & & \\
\hline & b & 20,7 & 20,8 & 20,8 & 20,77 & 20,6 & 20,7 & 20,7 & 20,67 & & & & & & & & & & \\
\hline \multirow[t]{3}{*}{5} & $\mathbf{L}$ & 93,5 & 93,6 & 93,5 & 93,53 & 93,5 & 93,6 & 93,5 & 93,53 & 93,5 & 93,7 & 93,5 & 93,57 & 93,6 & 93,5 & 93,6 & 93,57 & \multirow{3}{*}{0,81} & \multirow{3}{*}{0,11} \\
\hline & $a$ & $-1,3$ & $-1,3$ & $-1,3$ & $-1,30$ & $-1,3$ & $-1,3$ & $-1,3$ & $-1,30$ & $-1,7$ & $-1,6$ & $-1,6$ & $-1,63$ & $-1,7$ & $-1,7$ & $-1,7$ & $-1,70$ & & \\
\hline & b & 21 & 21,2 & 21,2 & 21,13 & 21,1 & 21 & 21 & 21,03 & 21,6 & 22,1 & 21,9 & 21,87 & 21,7 & 21,8 & 21,5 & 21,67 & & \\
\hline \multirow[t]{3}{*}{6} & $L$ & 90,1 & 89,9 & 90 & 90,00 & 90,2 & 90,4 & 90,4 & 90,33 & 91,1 & 90,4 & 90,9 & 90,8 & 90,3 & 91,2 & 90,7 & 90,73 & \multirow{3}{*}{0,94} & \\
\hline & $\mathbf{a}$ & $-1,2$ & $-1,2$ & $-1,2$ & $-1,20$ & $-1,2$ & $-1,2$ & $-1,2$ & $-1,20$ & $-1,7$ & $-1,6$ & $-1,6$ & $-1,63$ & $-1,7$ & $-1,7$ & $-1,7$ & $-1,70$ & & $-0,23$ \\
\hline & b & 19,6 & 20,1 & 19,9 & 19,87 & 19,9 & 20 & 20 & 19,97 & 20,2 & 20 & 20,1 & 20,10 & 20 & 20 & 20,1 & 20,03 & & \\
\hline 7 & $\mathbf{L}$ & 90,8 & 90,7 & 90,7 & 90,73 & 90,9 & 90,9 & 90,9 & 90,90 & 93,1 & 93,3 & 93,2 & 93,20 & 93,4 & 93,3 & 93,6 & 93,43 & & \\
\hline & $a$ & $-0,6$ & $-0,5$ & $-0,5$ & $\begin{array}{l}-0,53 \\
\end{array}$ & $-0,5$ & $-0,5$ & $-0,6$ & 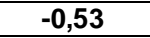 & \begin{tabular}{|l|}
$-1,3$ \\
\end{tabular} & $-1,3$ & $-1,2$ & $-1,27$ & $-0,6$ & $\begin{array}{l}-0,5 \\
\end{array}$ & $-0,6$ & $-0,57$ & 3,22 & 0,07 \\
\hline & b & 22,5 & 22,7 & 22,6 & 22,60 & 22,7 & 22,6 & 22,4 & 22,57 & 21,3 & 22,2 & 22,3 & 21,93 & 24,9 & 24,4 & 24,9 & 27,73 & & \\
\hline 8 & $L$ & 91,7 & 91,7 & 92 & 91,8 & 91,8 & 92,4 & 92,1 & 92,10 & 90,7 & 90,8 & 90,2 & 90,57 & 91,3 & 91 & 91,6 & 91,30 & & \\
\hline & $a$ & $-1,1$ & $\begin{array}{l}-1,1 \\
\end{array}$ & $-1,1$ & $-1,10$ & -1 & $-1,1$ & $-1,1$ & $-1,07$ & $-1,3$ & $-1,3$ & $-1,2$ & $-1,27$ & $-1,3$ & $-1,3$ & $-1,3$ & $-1,30$ & 1,32 & 0,49 \\
\hline & b & 21,4 & 21,5 & 21,6 & 21,50 & 21,5 & 21,7 & 21,6 & 21,60 & 21,3 & 22,2 & 22,3 & 21,93 & 22 & 22,4 & 22,4 & 22,27 & & \\
\hline 9 & $L$ & 90,8 & 81 & 81,2 & 91,00 & 91,1 & 91,2 & 91,3 & 91,20 & 91,2 & 91,3 & 91,2 & 91,23 & 91,5 & 91,2 & 91,2 & 91,30 & & \\
\hline & $a$ & -1 & -1 & $-1,1$ & $\begin{array}{l}1,03 \\
\end{array}$ & -1 & -1 & -1 & $-1,00$ & $-1,2$ & $\begin{array}{l}-1,2 \\
\end{array}$ & $\begin{array}{l}-12 \\
\end{array}$ & $-1,20$ & $-1,2$ & $\begin{array}{l}-1,2 \\
\end{array}$ & $-1,2$ & $-1,20$ & 0,47 & 0,19 \\
\hline & b & 20,9 & 21,2 & 20,8 & 20,97 & 21 & 21 & 21,1 & 21,03 & 21,5 & 21,4 & 21,1 & 21,33 & 22,2 & 21,7 & 21,3 & 21,73 & & \\
\hline & $\mathbf{L}$ & 92,1 & 92 & 92 & 92,03 & 92,1 & 92,2 & 92,1 & 92,13 & 93,3 & 93,3 & 93,6 & 93,40 & 93,4 & 93,4 & 94,5 & 93,77 & & \\
\hline 10 & $\mathbf{a}$ & $-1,2$ & $-1,2$ & $-1,2$ & $-1,20$ & $-1,2$ & $-1,2$ & $-1,2$ & $-1,20$ & $-1,5$ & $-1,5$ & $-1,5$ & $-1,50$ & $-1,5$ & $-1,5$ & $-1,7$ & $-1,57$ & 2,23 & 0,46 \\
\hline & b & 21,3 & 21,1 & 21,3 & 21,23 & 21,4 & 21,5 & 21,3 & 21,40 & 22,9 & 23 & 23 & 22,97 & 22,9 & 21,7 & 21,3 & 21,73 & & \\
\hline
\end{tabular}


Quadro 20- Leituras de cor das amostras do Cimento fotoativado (grupo controle)

\begin{tabular}{|c|c|c|c|c|c|c|c|c|c|c|c|c|c|c|c|c|c|c|c|}
\hline \multicolumn{20}{|c|}{ Controle Variolink II } \\
\hline \multirow{2}{*}{$\begin{array}{c}\text { Corpo de } \\
\text { Prova }\end{array}$} & \multirow{2}{*}{ EIXO } & \multicolumn{4}{|c|}{ LEITURA INICIA Branco } & \multicolumn{4}{|c|}{ LEITURA INICIAL Preto } & \multicolumn{4}{|c|}{ LEITURA Final Branco } & \multicolumn{4}{|c|}{ LEITURA FINAL Preto } & \multicolumn{2}{|c|}{$\Delta$} \\
\hline & & LI1 & LI2 & LI3 & MEDIA LI & LF1 & LF2 & LF3 & MEDIA LF & LI1 & LI2 & LI3 & MEDIA LI & LF1 & LF2 & LF3 & MEDIA LF & $\Delta \mathrm{E}$ & $\Delta \mathrm{T}$ \\
\hline \multirow{3}{*}{1} & $\mathbf{L}$ & 100,2 & 100,4 & 99,8 & 100,13 & 76,9 & 77 & 77 & 76,96 & & & & & & & & & & \\
\hline & $\mathbf{a}$ & $-4,9$ & -5 & $-4,5$ & $-4,80$ & $-5,2$ & $-5,3$ & $-5,3$ & $-5,26$ & & & & & & & & & & \\
\hline & b & 8,8 & 8,9 & 8,8 & 8,83 & 3,2 & 3,2 & 3,2 & 3,2 & & & & & & & & & & \\
\hline \multirow{3}{*}{2} & $\mathbf{L}$ & 101 & 101,2 & 101,2 & 101,13 & 76,2 & 76,3 & 76,3 & 76,26 & & & & & & & & & & \\
\hline & $\mathbf{a}$ & $-5,2$ & $-5,3$ & $-5,3$ & $-5,27$ & $-5,2$ & $-5,1$ & $-5,2$ & $-5,16$ & & & & & & & & & & \\
\hline & b & 10,9 & 9,9 & 9,9 & 10,03 & 3 & 3 & 3,1 & 3,03 & & & & & & & & & & \\
\hline \multirow{3}{*}{3} & $\mathbf{L}$ & 100,7 & 100,7 & 100,9 & 100,77 & 76 & 75,9 & 75,9 & 75,93 & & & & & & & & & & \\
\hline & a & $-5,4$ & $-5,5$ & $-5,5$ & $-5,47$ & $-5,3$ & $-5,3$ & $-5,3$ & $-5,3$ & & & & & & & & & & \\
\hline & b & 8,5 & 8,6 & 8,7 & 8,60 & 2,7 & 2,9 & 2,7 & 2,76 & & & & & & & & & & \\
\hline \multirow{3}{*}{4} & L & 101 & 100,9 & 100,9 & 100,93 & 76,1 & 75,8 & 76 & 75,96 & & & & & & & & & & \\
\hline & $a$ & $-4,9$ & $-4,9$ & $-4,9$ & $-4,90$ & $\begin{array}{l}-5,3 \\
\end{array}$ & $-5,3$ & $\begin{array}{l}-5,3 \\
\end{array}$ & $-5,3$ & & & & & & & & & & \\
\hline & b & 11,7 & 11,6 & 11,5 & 11,60 & 2,9 & 2,6 & 2,6 & 2,7 & & & & & & & & & & \\
\hline \multirow{3}{*}{5} & L & 101,7 & 102,1 & 101,9 & 101,9 & 76 & 75,9 & 76,2 & 76,03 & & & & & & & & & & \\
\hline & $\mathbf{a}$ & -51 & $-5,2$ & $-5,3$ & $-5,20$ & $-5,3$ & $-5,3$ & $-5,3$ & $-5,3$ & & & & & & & & & & \\
\hline & b & 8,6 & 8,4 & 8,6 & 8,53 & 2,7 & 2,7 & 2,7 & 2,7 & & & & & & & & & & \\
\hline \multirow{3}{*}{6} & L & 101,1 & 101 & 101,4 & 101,17 & 75,8 & 75,5 & 75,6 & 75,63 & & & & & & & & & & \\
\hline & $a$ & $-4,2$ & -4 & $-4,1$ & $-4,1$ & $-5,6$ & $-5,5$ & $-5,5$ & $-5,53$ & & & & & & & & & & \\
\hline & b & 12,6 & 12,9 & 12,9 & 12,80 & 2,2 & 2,1 & 2,2 & 2,16 & & & & & & & & & & \\
\hline \multirow{3}{*}{7} & $\mathbf{L}$ & 103,1 & 102,2 & 102,8 & 102,70 & 76,3 & 76,3 & 76,1 & 76,23 & & & & & & & & & & \\
\hline & $\mathbf{a}$ & -5 & $-4,9$ & -5 & $-4,97$ & $-5,3$ & $-5,3$ & $-5,2$ & $-5,26$ & & & & & & & & & & \\
\hline & b & 9,8 & 9,7 & 10 & 9,83 & 3,1 & 3,2 & 3,1 & 3,13 & & & & & & & & & & \\
\hline \multirow{3}{*}{8} & $\mathbf{L}$ & 101,4 & 101,6 & 101,4 & 101,47 & 75 & 74,7 & 74,9 & 74,86 & & & & & & & & & & \\
\hline & $a$ & -5 & -5 & $-4,9$ & $-4,97$ & $-5,2$ & $-5,2$ & $-5,2$ & $-5,2$ & & & & & & & & & & \\
\hline & b & 8,6 & 8,6 & 8,5 & 8,57 & 1,8 & 1,7 & 1,7 & 1,73 & & & & & & & & & & \\
\hline \multirow{3}{*}{9} & L & 102 & 101,8 & 101,9 & 101,90 & 76,1 & 76,1 & 76,1 & 76,1 & & & & & & & & & & \\
\hline & $a$ & $-5,2$ & $-5,2$ & $-5,3$ & $-5,23$ & $-5,2$ & $-5,2$ & $-5,1$ & $-5,26$ & & & & & & & & & & \\
\hline & b & 8,8 & 8,8 & 8,9 & 8,83 & 2,8 & 2,8 & 2,8 & 2,8 & & & & & & & & & & \\
\hline \multirow{3}{*}{10} & $\mathbf{L}$ & 103,1 & 103 & 103 & 103,03 & 74,5 & 74,5 & 74,6 & 74,53 & & & & & & & & & & \\
\hline & $a$ & $-4,8$ & $-4,8$ & $-4,8$ & $-4,80$ & $-5,2$ & $-5,3$ & $-5,3$ & $-5,26$ & & & & & & & & & & \\
\hline & b & 9,4 & 9,5 & 9,5 & 9,47 & 1,5 & 1,5 & 1,5 & 1,5 & & & & & & & & & & \\
\hline
\end{tabular}


Quadro 21- Leituras de cor das amostras do Cimento autoadesivo (grupo controle).

\begin{tabular}{|c|c|c|c|c|c|c|c|c|c|c|c|c|c|c|c|c|c|c|c|}
\hline \multicolumn{20}{|c|}{ Controle Rely-X U200 } \\
\hline \multirow{2}{*}{$\begin{array}{l}\text { Corpo de } \\
\text { Prova }\end{array}$} & \multirow{2}{*}{ EIXO } & \multicolumn{4}{|c|}{ LEITURA INICIA Branco } & \multicolumn{4}{|c|}{ LEITURA INICIAL Preto } & \multicolumn{4}{|c|}{ LEITURA Final Branco } & \multicolumn{4}{|c|}{ LEITURA FINAL Preto } & \multicolumn{2}{|c|}{$\Delta$} \\
\hline & & LI1 & LI2 & LI3 & MEDIA LI & LF1 & LF2 & LF3 & MEDIA LF & LI1 & LI2 & LI3 & MEDIA LI & LF1 & LF2 & LF3 & MEDIA LF & $\Delta \mathrm{E}$ & $\Delta \mathrm{T}$ \\
\hline \multirow{3}{*}{1} & $\mathbf{L}$ & 96,9 & 96,4 & 96,4 & 96,47 & 71,7 & 71,6 & 71,4 & 71,57 & & & & & & & & & & \\
\hline & a & $-4,2$ & $-3,9$ & -4 & $-4,03$ & $-3,6$ & $-3,7$ & $-3,7$ & $-3,67$ & & & & & & & & & & \\
\hline & b & 8,7 & 8,8 & 9 & 8,83 & $-0,1$ & $-0,5$ & $-0,3$ & $-0,30$ & & & & & & & & & & \\
\hline \multirow{3}{*}{2} & $\mathbf{L}$ & 94 & 94,5 & 94 & 94,17 & 67,8 & 67,3 & 67,3 & 67,47 & & & & & & & & & & \\
\hline & a & -4 & -4 & $-4,1$ & $-4,03$ & $-3,2$ & $-3,1$ & $-3,2$ & $-3,17$ & & & & & & & & & & \\
\hline & b & 7,2 & 7,2 & 7,2 & 7,20 & -2 & $-2,5$ & $-2,5$ & $-2,33$ & & & & & & & & & & \\
\hline \multirow{3}{*}{3} & $\mathbf{L}$ & 97,1 & 96,4 & 97 & 96,83 & 76,4 & 76,8 & 76,7 & 76,63 & & & & & & & & & & \\
\hline & $\mathbf{a}$ & $-3,1$ & $-3,1$ & $-3,1$ & $-3,13$ & $-2,8$ & $-2,9$ & $-2,9$ & $-2,87$ & & & & & & & & & & \\
\hline & b & 13,3 & 13 & 13,3 & 13,20 & 4,4 & 4,6 & 4,5 & 4,50 & & & & & & & & & & \\
\hline \multirow{3}{*}{4} & $\mathbf{L}$ & 89,2 & 89,7 & 89,7 & 89,53 & 68,6 & 69,2 & 68,4 & 68,73 & & & & & & & & & & \\
\hline & $a$ & $-3,6$ & $-3,6$ & $-3,9$ & $-3,13$ & $-3,5$ & $-3,5$ & $-3,4$ & $-3,47$ & & & & & & & & & & \\
\hline & b & 8,7 & 8,8 & 8,7 & 8,73 & $-0,3$ & 0 & $-0,3$ & $-0,20$ & & & & & & & & & & \\
\hline \multirow{3}{*}{5} & L & 99,5 & 99,3 & 98,6 & 99,13 & 75,3 & 75,2 & 75,7 & 75,40 & & & & & & & & & & \\
\hline & $\mathbf{a}$ & $-3,9$ & $-3,8$ & $-3,9$ & $-3,87$ & $-3,3$ & $-3,2$ & $-3,1$ & $-3,20$ & & & & & & & & & & \\
\hline & b & 11,6 & 11,7 & 11,3 & 11,53 & 2,8 & 2,7 & 3,2 & 2,90 & & & & & & & & & & \\
\hline \multirow{3}{*}{6} & $\mathbf{L}$ & 98,6 & 97,5 & 96,8 & 97,63 & 76,3 & 75,8 & 75,6 & 75,90 & & & & & & & & & & \\
\hline & $a$ & -39 & $-3,9$ & $-3,9$ & $-3,90$ & $-3,4$ & $-3,4$ & $-3,4$ & $-3,40$ & & & & & & & & & & \\
\hline & b & 13,2 & 12,7 & 12,7 & 12,87 & 4 & 3,9 & 3,9 & 3,93 & & & & & & & & & & \\
\hline \multirow{3}{*}{7} & L & 95,9 & 94,6 & 95,5 & 95,33 & 73,8 & 73,4 & 73,4 & 73,53 & & & & & & & & & & \\
\hline & $a$ & $-3,7$ & $-3,2$ & $-3,5$ & $-3,47$ & $-3,2$ & $-3,2$ & $-3,2$ & $-3,20$ & & & & & & & & & & \\
\hline & b & 12 & 11,8 & 12 & 11,93 & 3,4 & 3,2 & 3,1 & 3,23 & & & & & & & & & & \\
\hline \multirow{3}{*}{8} & $\mathbf{L}$ & 99,8 & 99,6 & 99,1 & 99,50 & 76,2 & 76,5 & 78 & 76,90 & & & & & & & & & & \\
\hline & $a$ & $-4,5$ & $-4,6$ & $-4,6$ & $-4,57$ & $-3,9$ & $-3,8$ & $-3,5$ & $-3,73$ & & & & & & & & & & \\
\hline & b & 11,6 & 11,5 & 11,4 & 11,50 & 2,5 & 2,6 & 3,6 & 2,90 & & & & & & & & & & \\
\hline \multirow{3}{*}{9} & L & 94,5 & 94,2 & 93,8 & 94,50 & 70,4 & 70,4 & 69,9 & 70,23 & & & & & & & & & & \\
\hline & $\mathbf{a}$ & $-4,4$ & -4 & $-3,8$ & $-4,07$ & $-3,6$ & $-3,3$ & $-3,4$ & $-3,43$ & & & & & & & & & & \\
\hline & b & 8,6 & 8 & 8,1 & 8,23 & $-1,1$ & $-0,9$ & $-1,2$ & $-1,07$ & & & & & & & & & & \\
\hline \multirow{3}{*}{10} & $\mathbf{L}$ & 88,4 & 88,9 & 88,3 & 88,53 & 61,7 & 61,8 & 61 & 61,50 & & & & & & & & & & \\
\hline & $\mathbf{a}$ & $-3,9$ & $-3,7$ & $-3,7$ & $-3,77$ & $-3,1$ & $-3,1$ & $-3,1$ & $-3,10$ & & & & & & & & & & \\
\hline & b & 4,9 & 5,2 & 5 & 5,03 & $-5,5$ & $-5,3$ & $-5,7$ & $-5,50$ & & & & & & & & & & \\
\hline
\end{tabular}


Quadro 22- Leituras de cor das amostras dos Dentes (grupo controle)

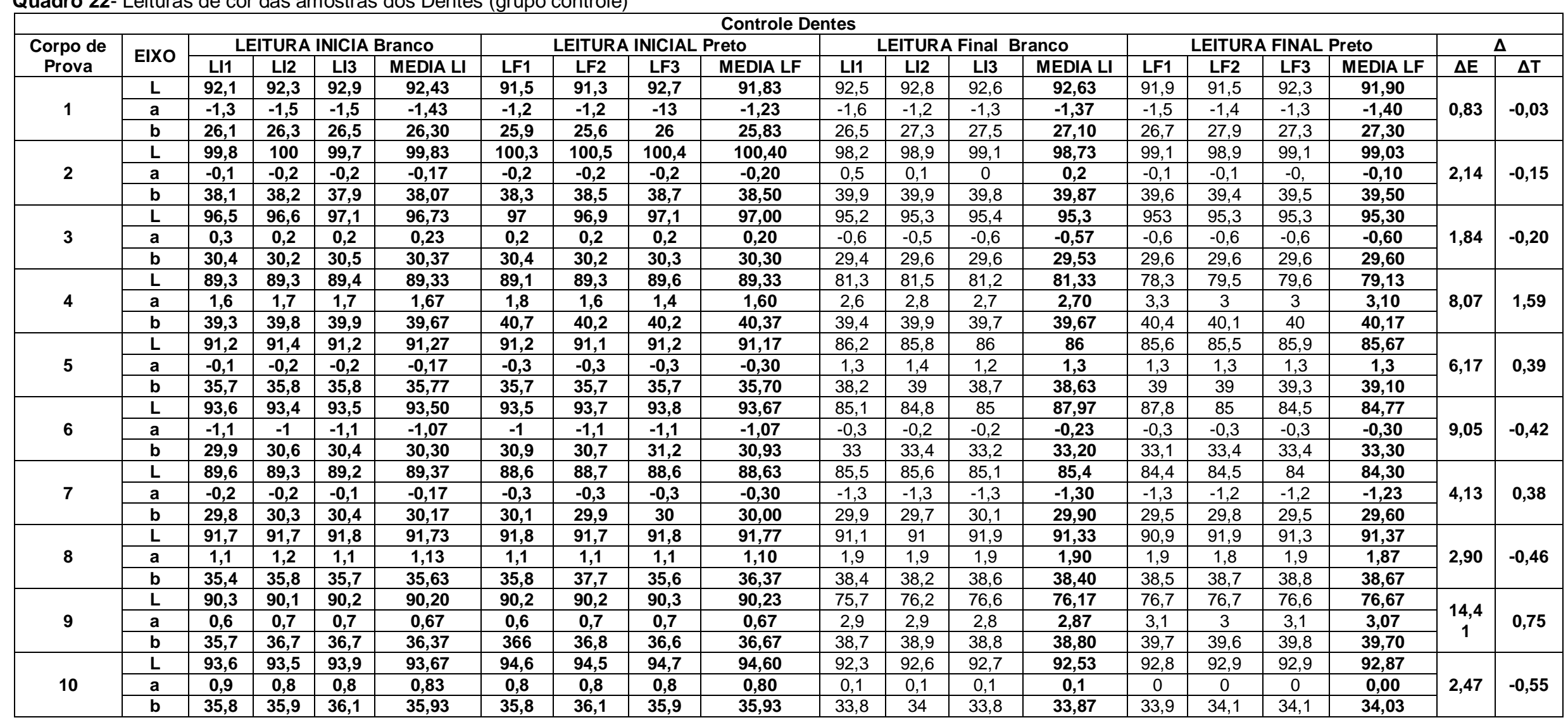


Os quadros 23 ao 33 constam as leituras iniciais de cor, sobre fundo preto e fundo branco, dos corpos de prova que não foram submetidos a nenhum tratamento de envelhecimento.

Quadro 23- Leituras de cor das amostras de E-max Press na espessura de 0,5mm cimentados com cimento fotoativado.

\begin{tabular}{|c|c|c|c|c|c|c|c|c|c|c|c|c|c|c|c|c|c|c|c|}
\hline \multicolumn{20}{|c|}{ E-max Press 0,5mm Variolink II } \\
\hline \multirow{2}{*}{$\begin{array}{l}\text { Corpo de } \\
\text { Prova }\end{array}$} & \multirow{2}{*}{ EIXO } & \multicolumn{4}{|c|}{ LEITURA INICIA Branco } & \multicolumn{4}{|c|}{ LEITURA INICIAL Preto } & \multicolumn{4}{|c|}{ LEITURA Final Branco } & \multicolumn{4}{|c|}{ LEITURA FINAL Preto } & \multicolumn{2}{|c|}{$\Delta$} \\
\hline & & LI1 & LI2 & LI3 & MEDIA LI & LF1 & LF2 & LF3 & MEDIA LF & LI1 & LI2 & $\mathrm{LI} 3$ & MEDIA LI & LF1 & LF2 & LF3 & MEDIA LF & $\Delta \mathrm{E}$ & $\Delta \mathbf{T}$ \\
\hline \multirow{3}{*}{1} & $\mathbf{L}$ & 90,5 & 90,2 & 90 & 90,23 & 88,9 & 90,6 & 90,2 & 90,23 & 92,5 & 92,2 & 92,9 & 92,53 & 92,4 & 92,6 & 92,8 & 92,60 & \multirow{3}{*}{2,36} & \multirow{3}{*}{$-0,49$} \\
\hline & $\mathbf{a}$ & $-1,9$ & $-1,8$ & $-1,9$ & $-1,87$ & $-1,7$ & $-2,1$ & $-1,9$ & $-1,90$ & $-1,8$ & $-1,8$ & $-1,8$ & $-1,80$ & $\begin{array}{l}-1,8 \\
\end{array}$ & $\begin{array}{l}-1,8 \\
\end{array}$ & $-1,8$ & $-1,80$ & & \\
\hline & B & 15,9 & 17 & 16,4 & 16,43 & 16,5 & 15,4 & 15,7 & 15,87 & 15,9 & 16 & 15,8 & 15,90 & 16 & 15,9 & 15,7 & 15,87 & & \\
\hline \multirow{3}{*}{2} & $\mathbf{L}$ & 87,6 & 87,2 & 88 & 87,6 & 87,5 & 87,7 & 87,5 & 87,57 & 94,7 & 94,5 & 94,9 & 94,70 & 94,6 & 94,6 & 94,8 & 94,67 & \multirow{3}{*}{7,12} & \multirow{3}{*}{0,48} \\
\hline & $a$ & $-0,4$ & $-0,4$ & $-0,3$ & $-0,37$ & $-0,4$ & $-0,5$ & $-0,4$ & $-0,43$ & $-0,6$ & $-0,6$ & $-0,6$ & $-0,60$ & $-0,6$ & $-0,5$ & $-0,6$ & $-0,57$ & & \\
\hline & b & 24,1 & 24,2 & 24,2 & 24,17 & 23,9 & 24,3 & 24 & 24,07 & 24,1 & 23,5 & 23,6 & 23,73 & 24,5 & 24,3 & 24,2 & 24,33 & & \\
\hline \multirow{3}{*}{3} & L & 94,7 & 96 & 94,9 & 95,2 & 94,9 & 94,8 & 95,3 & 95,00 & 94,7 & 95,5 & 94,7 & 94,97 & 94,9 & 94,8 & 95,3 & 95,00 & \multirow{3}{*}{0,24} & \multirow{3}{*}{$-0,10$} \\
\hline & $\mathbf{a}$ & $-1,7$ & $-1,7$ & $-1,8$ & $-1,73$ & $-1,7$ & $-1,7$ & $-1,7$ & $-1,70$ & $-1,7$ & $-1,7$ & $-1,6$ & $-1,67$ & $-1,7$ & -17, & $\begin{array}{l}-1,7 \\
\end{array}$ & $-1,70$ & & \\
\hline & b & 19 & 18,9 & 19,2 & 19,03 & 19 & 18,7 & 19 & 18,90 & 19 & 18,9 & 19,2 & 19,03 & 19 & 18,7 & 19 & 1890 & & \\
\hline \multirow{3}{*}{4} & $\mathbf{L}$ & 98,7 & 98,5 & 99,1 & 98,77 & 98,7 & 99,1 & 98,4 & 98,73 & 98,7 & 98,1 & 99,1 & 98,63 & 98,7 & 99,1 & 98,4 & 98,73 & \multirow{3}{*}{0,15} & \multirow{3}{*}{0,05} \\
\hline & $a$ & $-0,5$ & $-0,4$ & $-0,6$ & $-0,50$ & $-0,4$ & $-0,5$ & $-0,4$ & $-0,43$ & $-0,5$ & $-0,4$ & $\begin{array}{l}-0,5 \\
\end{array}$ & $-0,47$ & $-0,4$ & $-0,5$ & $-0,4$ & $-0,43$ & & \\
\hline & b & 24,1 & 24 & 24 & 24,03 & 24,1 & 24,1 & 23,9 & 24,03 & 23,9 & 24 & 24 & 23,97 & 24,1 & 24,1 & 23,9 & 24,03 & & \\
\hline \multirow{3}{*}{5} & $\mathbf{L}$ & 90,3 & 91 & 90,5 & 90,60 & 90,8 & 90,3 & 90,1 & 90,40 & 96,3 & 96,5 & 96,7 & 96,50 & 96,5 & 96,6 & 96,4 & 96,50 & \multirow{3}{*}{6,13} & \multirow{3}{*}{0,53} \\
\hline & $\mathbf{a}$ & $-1,2$ & $-1,3$ & $-1,2$ & $-1,23$ & $-1,2$ & $-1,1$ & $-1,1$ & $-1,13$ & $-1,5$ & $-1,5$ & $-1,5$ & $-1,50$ & $-1,6$ & $-1,6$ & $-1,6$ & $-1,60$ & & \\
\hline & b & 19,6 & 19,3 & 19,7 & 19,53 & 19,6 & 19,6 & 19,7 & 19,63 & 21 & 21,2 & 21,3 & 21,17 & 20,3 & 20,4 & 20,5 & 20,40 & & \\
\hline & $L$ & 92,1 & 92 & 92 & 92,03 & 91,8 & 91,9 & 919 & 91,87 & 92 & 91,6 & 92 & 91,87 & 91,8 & 91,9 & 91,9 & 91,87 & & \\
\hline 6 & $a$ & 0,3 & 0,4 & 0,3 & 0,33 & 0,3 & 0,3 & 0,3 & 0,30 & 0,3 & 0,3 & 0,3 & 0,30 & 0,3 & 0,3 & 0,3 & 0,30 & 0,17 & $-0,03$ \\
\hline & b & 26,1 & 26,1 & 26,1 & 26,10 & 25,3 & 25,9 & 25,9 & 25,70 & 26,1 & 26,1 & 26,1 & 26,10 & 25,3 & 25,9 & 25,9 & 25,70 & & \\
\hline & $L$ & 91,7 & 91,5 & 91,7 & 91,63 & 91,9 & 91,6 & 91,4 & 91,63 & 99,3 & 98,8 & 99,1 & 99,07 & 99,6 & 99 & 99,2 & 99,27 & & \\
\hline 7 & $\mathbf{a}$ & $-1,1$ & $-1,1$ & -1 & $-1,07$ & -1 & $-1,1$ & $-1,1$ & $-1,07$ & $-1,1$ & $-1,2$ & $\begin{array}{l}-1,3 \\
\end{array}$ & $-1,20$ & $-1,3$ & $-1,1$ & $-1,3$ & $-1,23$ & 7,49 & 0,26 \\
\hline & b & 19,7 & 20,1 & 19,7 & 19,83 & 19,7 & 19,8 & 20 & 19,83 & 21,2 & 20,7 & 20,4 & 20,77 & 20,3 & 21,2 & 20,3 & 20,60 & & \\
\hline & $L$ & 92,4 & 93 & 92,9 & 92,77 & 93,4 & 91,5 & 92,2 & 92,37 & 93,7 & 93,7 & 94,8 & 94,07 & 94,4 & 94,9 & 93,2 & 94,17 & & \\
\hline 8 & $a$ & $-0,5$ & $-0,6$ & $-0,5$ & $-0,53$ & $-0,7$ & $-0,2$ & $-0,3$ & $-0,40$ & $-0,8$ & $-0,9$ & $-1,1$ & $-0,93$ & $\begin{array}{l}-0,8 \\
\end{array}$ & $-1,1$ & $-0,9$ & $-0,93$ & 1,43 & $-0,38$ \\
\hline & b & 20,4 & 20,5 & 20,4 & 20,43 & 20,2 & 20 & 20,2 & 20,13 & 20,5 & 21 & 21,1 & 20,87 & 20,9 & 21 & 21 & 20,97 & & \\
\hline & $L$ & 91,9 & 91,9 & 91,8 & 91,87 & 92,3 & 91,6 & 91,3 & 91,73 & 93,4 & 93,3 & 93,6 & 93,43 & 93,1 & 93,1 & 93,1 & 93,10 & & \\
\hline 9 & $a$ & $-0,1$ & $-0,2$ & $-0,1$ & $-0,13$ & $-0,3$ & $-0,2$ & $-0,1$ & $-0,20$ & $\begin{array}{l}-1 \\
\end{array}$ & $-0,8$ & $-0,9$ & $-0,90$ & $-0,8$ & $-0,9$ & $-0,9$ & $-0,87$ & 1,83 & 0,23 \\
\hline & b & 19,6 & 19,6 & 19,6 & 19,60 & 19,8 & 19,7 & 19,5 & 19,67 & 20,1 & 20,1 & 20,3 & 20,17 & 20 & 19,9 & 20 & 19,97 & & \\
\hline & $\mathbf{L}$ & 91,5 & 92 & 92,9 & 92,13 & 93,3 & 92,3 & 91,7 & 92,43 & 93,1 & 93 & 93,4 & 93,17 & 93,4 & 93,9 & 92,7 & 93,33 & & \\
\hline 10 & $a$ & $-0,9$ & $-0,9$ & $-0,9$ & $-0,90$ & -1 & $-1,1$ & $-1,2$ & $-1,10$ & $-1,5$ & $-1,5$ & $-1,6$ & $-1,53-$ & $-1,4$ & $-1,6$ & $-1,6$ & $-1,47$ & 1,22 & $-0,16$ \\
\hline & b & 17,1 & 17,6 & 16,9 & 17,20 & 17,3 & 17,2 & 17 & 17,17 & 17 & 17,7 & 17,4 & 17,37 & 17,1 & 17,3 & 17,4 & 17,27 & & \\
\hline
\end{tabular}


Quadro 24- Leituras de cor das amostras de E-max Press na espessura de 1,0mm cimentados com cimento fotoativado.

\begin{tabular}{|c|c|c|c|c|c|c|c|c|c|c|c|c|c|c|c|c|c|c|c|}
\hline \multicolumn{20}{|c|}{ E-max Press 1,0mm Variolink II } \\
\hline \multirow{2}{*}{$\begin{array}{c}\text { Corpo de } \\
\text { Prova }\end{array}$} & \multirow{2}{*}{ EIXO } & \multicolumn{4}{|c|}{ LEITURA INICIAL Branco } & \multicolumn{4}{|c|}{ LEITURA INICIAL Preto } & \multicolumn{4}{|c|}{ LEITURA FINAL Branco } & \multicolumn{4}{|c|}{ LEITURA FINAL Preto } & \multicolumn{2}{|c|}{$\Delta$} \\
\hline & & LI1 & LI2 & LI3 & MEDIA LI & LF1 & LF2 & LF3 & MEDIA LF & LI1 & LI2 & LI3 & MEDIA LI & LF1 & LF2 & LF3 & MEDIA LF & $\Delta \mathrm{E}$ & $\Delta \mathrm{T}$ \\
\hline \multirow{3}{*}{. } & $\mathbf{L}$ & 91 & 9,6 & 91,4 & 91,33 & 91,3 & 91,8 & 916 & 91,57 & 91,6 & 91,2 & 918 & 91,53 & 92,2 & 92,3 & 92 & 92,17 & \multirow{3}{*}{0,92} & \multirow{3}{*}{0,38} \\
\hline & $\mathbf{a}$ & -1 & -1 & -1 & $-1,00$ & $-1,1$ & $-1,1$ & $-1,1$ & $-1,10$ & -1 & $-0,9$ & -1 & $-0,97$ & -1 & -1 & -1 & $-1,00$ & & \\
\hline & b & 17,6 & 17,6 & 17,8 & 17,67 & 17,7 & 17,7 & 17,7 & 17,70 & 18,6 & 18,6 & 18,5 & 18,57 & 18,5 & 18,6 & 18,6 & 18,57 & & \\
\hline \multirow{3}{*}{2} & $\mathbf{L}$ & 93,3 & 92,9 & 93,2 & 93,13 & 93 & 93,1 & 93,3 & 93,13 & 93,3 & 93,5 & 94 & 93,6 & 93,6 & 93,7 & 93,7 & 93,67 & \multirow{3}{*}{0,79} & \multirow{3}{*}{$-0,01$} \\
\hline & $a$ & $-1,2$ & $-1,1$ & $-1,1$ & $-1,13$ & $-1,1$ & $-1,1$ & $-1,1$ & $-1,10$ & $-1,1$ & $-1,2$ & $-1,2$ & $-1,17$ & $-1,1$ & $-1,1$ & $-1,1$ & $-1,10$ & & \\
\hline & b & 17,2 & 17,5 & 17,6 & 17,43 & 17,6 & 17,5 & 17,5 & 17,53 & 18,1 & 18,2 & 17,9 & 18,07 & 18,2 & 18,1 & 18 & 18,10 & & \\
\hline \multirow{3}{*}{3} & $\mathbf{L}$ & 91,4 & 91,7 & 91,1 & 91,40 & 92,2 & 91,7 & 92 & 91,97 & 92 & 91,7 & 92,3 & 92,00 & 92,4 & 92,5 & 91,7 & 92,20 & \multirow{3}{*}{0,87} & \multirow{3}{*}{$-0,34$} \\
\hline & $a$ & $-0,5$ & $-0,5$ & $-0,5$ & $-0,50$ & $-0,5$ & $-0,6$ & $-0,5$ & $-0,53$ & $-0,5$ & $-0,5$ & $-0,5$ & $-0,50$ & $-0,5$ & $-0,5$ & $-0,5$ & $-0,50$ & & \\
\hline & b & 18,8 & 18,8 & 18,6 & 18,73 & 19,1 & 18,7 & 18,7 & 18,83 & 19,2 & 19,4 & 19,5 & 1937 & 19,6 & 19,5 & 19,4 & 19,50 & & \\
\hline \multirow{3}{*}{4} & $\mathbf{L}$ & 91,2 & 91,2 & 91 & 91,13 & 91,5 & 90,8 & 91,5 & 91,27 & 91,4 & 92 & 91,1 & 91,50 & 91,3 & 91,2 & 91,8 & 91,43 & \multirow{3}{*}{0,62} & \multirow{3}{*}{0} \\
\hline & $a$ & $-1,2$ & $-1,2$ & $-1,2$ & $-1,20$ & $-1,2$ & $-1,2$ & $-1,2$ & $-1,20$ & $-1,1$ & $-1,1$ & $-1,2$ & $-1,13$ & $-1,2$ & $-1,2$ & $-1,2$ & $-1,20$ & & \\
\hline & b & 17,3 & 17,3 & 17,3 & 17,30 & 17,3 & 17,1 & 17,1 & 17,17 & 17,7 & 18 & 17,7 & 17,80 & 17,6 & 17,6 & 17,7 & 17,63 & & \\
\hline \multirow{3}{*}{5} & $\mathbf{L}$ & 90,4 & 90,5 & 90,7 & 90,53 & 91 & 90,8 & 91,5 & 91,27 & 91,4 & 91,5 & 91,6 & 91,50 & 92,7 & 91,9 & 92,4 & 92,33 & \multirow{3}{*}{1,4} & \multirow{3}{*}{0,32} \\
\hline & $\mathbf{a}$ & $-0,9$ & $-0,8$ & $-0,8$ & $-0,83$ & $-0,8$ & $-0,8$ & $-0,8$ & $-0,80$ & $-0,7$ & $-0,7$ & $-0,7$ & $-0,70$ & $-0,8$ & $-0,8$ & $-0,8$ & $-0,80$ & & \\
\hline & b & 19,6 & 18,8 & 18,9 & 18,77 & 17,3 & 17,1 & 17,1 & 17,17 & 19,7 & 19,7 & 19,9 & 19,77 & 19,8 & 19,7 & 20 & 19,83 & & \\
\hline 6 & $\mathbf{L}$ & 92,8 & 92,8 & 92,8 & 92,80 & 91 & 90,9 & 90,9 & 90,93 & 93,8 & 93,9 & 93,7 & 93,80 & 93,5 & 93,7 & 94,2 & 93,8 & & \\
\hline 6 & $\mathbf{a}$ & $-0,8$ & $-0,8$ & $-0,8$ & $-0,80$ & $-0,8$ & $-0,8$ & $-0,8$ & $0-, 8$ & $-0,8$ & $-0,8$ & $-0,7$ & $-0,77$ & $-0,8$ & $-0,7$ & $-0,8$ & $-0,77$ & 1,26 & $-0,09$ \\
\hline & b & 20,3 & 20,3 & 20,4 & 20,33 & 19,1 & 19 & 19,2 & 19,10 & 21 & 21,1 & 21,2 & 21,10 & 21,2 & 21,2 & 21,2 & 21,20 & & \\
\hline & $\mathbf{L}$ & 94,1 & 93,2 & 93,5 & 93,60 & 92,7 & 92,6 & 92,7 & 92,67 & 94 & 94 & 93,5 & 93,83 & 93,4 & 94 & 93,6 & 93,67 & & \\
\hline 7 & $\mathbf{a}$ & $-1,1$ & $-0,9$ & -1 & -1 & $-0,7$ & $-0,7$ & $-0,7$ & $-0,70$ & -1 & -1 & $-0,9$ & $-0,97$ & $-0,9$ & -1 & -1 & $-0,97$ & 0,87 & $-0,44$ \\
\hline & b & 17,6 & 18 & 18,1 & 17,90 & 20,1 & 20,4 & 20,2 & 20,23 & 18,7 & 18,6 & 18,9 & 19,87 & 18,4 & 18,7 & 18,6 & 18,57 & & \\
\hline & $\mathbf{L}$ & 91,5 & 91,4 & 91,4 & 91,43 & 92,6 & 93,2 & 93 & 92,93 & 92,1 & 92,4 & 92,8 & 93,03 & 92,5 & 92,6 & 92,2 & 92,43 & & \\
\hline 8 & $\mathbf{a}$ & $-0,5$ & $-0,5$ & $-0,5$ & $-0,50$ & -1 & $-0,9$ & $-0,9$ & $-0,93$ & $-0,6$ & $-0,7$ & $-0,6$ & $\begin{array}{c}-0,63 \\
\end{array}$ & $-0,7$ & $-0,6$ & $-0,7$ & $-0,67$ & 0,8 & $-0,01$ \\
\hline & b & 19,8 & 19,6 & 19,7 & 19,70 & 17,9 & 17,9 & 17,7 & 17,83 & 20,2 & 19,9 & 19,9 & 20,00 & 20 & 19,9 & 20,1 & 20,00 & & \\
\hline & $\mathbf{L}$ & 91,4 & 92,2 & 92,3 & 92,30 & 91,4 & 92 & 91,5 & 91,63 & 93,4 & 92,9 & 92,8 & 93,03 & 93,4 & 93 & 93,3 & 93,23 & & \\
\hline 9 & $\mathbf{a}$ & $-0,9$ & -1 & -1 & $-0,97$ & $-0,5$ & $-0,5$ & $-0,5$ & $-0,50$ & -1 & -1 & -1 & $-1,00$ & -1 & -1 & -1 & $-1,00$ & 1,21 & $-0,11$ \\
\hline & b & 17,5 & 17,4 & 17,4 & 17,43 & 19,4 & 19,5 & 19,6 & 19,50 & 18,4 & 18,4 & 18,4 & 18,40 & 18,6 & 18,4 & 18,7 & 18,57 & & \\
\hline & $\mathbf{L}$ & 93,1 & 93,3 & 93,1 & 93,17 & 93,1 & 93,1 & 93,2 & 93,13 & 94,3 & 93,7 & 95,1 & 94,37 & 94,3 & 93,9 & 94 & 94,07 & & \\
\hline 10 & $a$ & $-0,9$ & $-0,9$ & $-0,9$ & $-0,90$ & $-0,9$ & $-0,9$ & $-0,9$ & $-0,90$ & -1 & $-0,9$ & -1 & $-0,97$ & -1 & -1 & -1 & $-1,00$ & 1,54 & 0,26 \\
\hline & b & 19,2 & 19,1 & 19,1 & 19,13 & 19,1 & 19,3 & 19,3 & 19,23 & 20,1 & 20,3 & 19,9 & 20,10 & 19,8 & 20 & 19,9 & 19,90 & & \\
\hline
\end{tabular}


Quadro 25- Leituras de cor das amostras de E-max Press na espessura de 0,5mm cimentados com cimento autoadesivo.

\begin{tabular}{|c|c|c|c|c|c|c|c|c|c|c|c|c|c|c|c|c|c|c|c|}
\hline \multicolumn{20}{|c|}{ E-max Press 0,5mm Rely-X U200 } \\
\hline \multirow{2}{*}{$\begin{array}{c}\text { Corpo } \\
\text { de Prova }\end{array}$} & \multirow[b]{2}{*}{ EIXO } & \multicolumn{4}{|c|}{ LEITURA INICIAL Branco } & \multicolumn{4}{|c|}{ LEITURA INICIAL Preto } & \multicolumn{4}{|c|}{ LEITURA Final Branco } & \multicolumn{4}{|c|}{ LEITURA FINAL Preto } & \multicolumn{2}{|c|}{$\Delta$} \\
\hline & & LI1 & LI2 & LI3 & MEDIA LI & LF1 & LF2 & LF3 & MEDIA LF & LI1 & LI2 & LI3 & MEDIA LI & LF1 & LF2 & LF3 & MEDIA LF & $\Delta \mathrm{E}$ & $\Delta T$ \\
\hline \multirow{3}{*}{1} & $\mathbf{L}$ & 90,8 & 90,9 & 91,1 & 90,93 & 89,8 & 90,8 & 90,6 & 90,40 & 89,5 & 88,8 & 88,8 & 89,03 & 89,2 & 89,3 & 89,7 & 89,40 & \multirow{3}{*}{3,16} & \multirow{3}{*}{$-0,31$} \\
\hline & $\mathbf{a}$ & 0,2 & 0,2 & 0,1 & 0,17 & 0,7 & 0,5 & 0,6 & 0,60 & 0,7 & 0,8 & 0,6 & 0,70 & 0,7 & 0,8 & 0,7 & 0,73 & & \\
\hline & b & 23 & 24,2 & 23,4 & 23,53 & 24 & 23,2 & 23,1 & 23,43 & 20,3 & 21,6 & 21,3 & 21,07 & 21,1 & 21,5 & 20,3 & 20,97 & & \\
\hline \multirow{3}{*}{2} & $\mathbf{L}$ & 91,7 & 92,1 & 91,6 & 91,80 & 92,3 & 92,7 & 92,2 & 92,40 & 91,5 & 90,8 & 91,5 & 91,27 & 90,9 & 90,6 & 91,1 & 90,87 & \multirow{3}{*}{0,80} & \multirow{3}{*}{$-0,27$} \\
\hline & $\mathbf{a}$ & $-0,1$ & $-0,2$ & 0,1 & $-0,07$ & $-0,3$ & $-0,3$ & $-0,3$ & $-0,30$ & 0,4 & 0,5 & 0,6 & 0,50 & 0,7 & 0,3 & 0,6 & 0,53 & & \\
\hline & b & 22,2 & 22,1 & 22,4 & 22,23 & 22,1 & 21,8 & 22,2 & 22,03 & 22,4 & 22,8 & 22 & 22,4 & 22,1 & 22,9 & 22,4 & 22,47 & & \\
\hline \multirow{3}{*}{3} & $\mathrm{~L}$ & 92,3 & 92,4 & 92,4 & 92,37 & 92,4 & 92,5 & 92,6 & 92,50 & 91,4 & 91,6 & 91,5 & 91,50 & 91,5 & 91,6 & 91,5 & 91,53 & \multirow{3}{*}{1,58} & \multirow{3}{*}{$-0,58$} \\
\hline & $\mathbf{a}$ & 0 & 0,2 & 0,2 & 0,13 & 0,3 & 0,2 & 0,2 & 0,23 & 1 & 1 & 1 & 1,00 & 0,9 & 0,9 & 0,9 & 0,90 & & \\
\hline & b & 25,2 & 25,7 & 25,9 & 25,6 & 26,4 & 26,3 & 26,1 & 26,27 & 26,6 & 26,5 & 26,7 & 26,60 & 26,5 & 26,5 & 26,7 & 26,57 & & \\
\hline \multirow{3}{*}{4} & L & 90,1 & 90,4 & 90,2 & 90,23 & 90,8 & 90,5 & 90,5 & 90,60 & 88,8 & 89 & 88,9 & 88,90 & 89,2 & 89,1 & 88,6 & 88,97 & \multirow{3}{*}{1,58} & \multirow{3}{*}{0,06} \\
\hline & $\mathbf{a}$ & $-1,4$ & $-1,3$ & -1 & $-1,23$ & $-0,9$ & $-0,8$ & $-0,9$ & $-0,87$ & -1 & -1 & $-0,9$ & $-0,97$ & $-0,8$ & $-0,8$ & $-0,9$ & $-0,83$ & & \\
\hline & b & 17,4 & 17,5 & 17,1 & 17,33 & 17,6 & 17,5 & 17,2 & 17,43 & 16,6 & 16,2 & 168 & 16,53 & 17 & 17,2 & 17,1 & 17,10 & & \\
\hline \multirow{3}{*}{5} & L & 92,7 & 89,9 & 89,7 & 90,77 & 90,2 & 91,5 & 92,2 & 91,30 & 89,5 & 89,8 & 89 & 89,43 & 89,3 & 89,1 & 88,8 & 88,07 & \multirow{3}{*}{5,26} & \multirow{3}{*}{$-0,15$} \\
\hline & $\mathbf{a}$ & $-2,1$ & $-2,1$ & -2 & $-2,07$ & $-1,9$ & $-1,9$ & $-1,9$ & $-1,90$ & 0 & 0,1 & 0 & 0,03 & 0 & 0 & 0,1 & 0,03 & & \\
\hline & b & 17 & 16,7 & 16,7 & 16,8 & 16,8 & 17,1 & 17,3 & 17,07 & 21,4 & 21,6 & 21,3 & 21,43 & 21,2 & 21,2 & 21 & 21,13 & & \\
\hline & L & 91,8 & 91,1 & 91,7 & 91,53 & 90,9 & 90,7 & 90,7 & 90,77 & 95,2 & 95,2 & 95,2 & 95,2 & 94,9 & 94,7 & 95,4 & 95,00 & & \\
\hline 6 & $a$ & $-0,6$ & $-0,6$ & $-0,7$ & $-0,63$ & $-0,4$ & $-0,5$ & $-0,6$ & $-0,50$ & $-0,2$ & $-0,2$ & $-0,2$ & $-0,20$ & $-0,1$ & $-0,1$ & $-0,2$ & $-0,13$ & 5,52 & $-0,98$ \\
\hline & b & 22,7 & 23,1 & 22,7 & 22,83 & 24,3 & 23,7 & 23,2 & 23,73 & 18,7 & 18,6 & 18,9 & 19,73 & 18,9 & 18,9 & 18,5 & 18,77 & & \\
\hline & $\mathrm{L}$ & 96,7 & 96,2 & 96,2 & 96,37 & 86,1 & 86,6 & 87 & 86,57 & 96 & 95,8 & 95,8 & 95,87 & 95,7 & 95,7 & 95,8 & 95,73 & & \\
\hline 7 & $\mathbf{a}$ & $-1,9$ & $-1,9$ & $-1,9$ & $-1,90$ & $-1,9$ & -2 & -2 & $-1,97$ & $-0,3$ & $-0,3$ & $-0,3$ & $-0,30$ & $-0,2$ & $-0,2$ & $-0,3$ & $-0,23$ & 3,61 & 9,62 \\
\hline & b & 18,8 & 18,5 & 18,5 & 18,60 & 18,6 & 18,3 & 18,7 & 18,53 & 21,8 & 21,9 & 21,7 & 21,80 & 21,7 & 22,1 & 21,9 & 21,90 & & \\
\hline & L & 95,5 & 95,5 & 95,9 & 95,63 & 96,1 & 95,9 & 955 & 95,83 & 93,7 & 93,8 & 94,1 & 93,87 & 94,3 & 94 & 94 & 94,1 & & \\
\hline 8 & $\mathbf{a}$ & $-0,7$ & $-0,7$ & $-0,7$ & $-0,70$ & $-0,7$ & $-0,7$ & $-0,7$ & $-0,70$ & $-0,2$ & $-0,2$ & $-0,2$ & $-0,20$ & $-0,3$ & $-0,3$ & $-0,3$ & $-0,30$ & 1,84 & $-0,32$ \\
\hline & b & 23,1 & 23,5 & 23,3 & 23,30 & 23,7 & 24,2 & 23,6 & 23,83 & 23,2 & 23,6 & 23,5 & 23,43 & 23,5 & 23,1 & 23,7 & 23,43 & & \\
\hline & $\mathrm{L}$ & 92,2 & 92,2 & 92,4 & 92,27 & 39,1 & 92,1 & 92,1 & 92,43 & 92,3 & 92,2 & 92,4 & 92,30 & 92,3 & 92,3 & 92,6 & 92,40 & & \\
\hline 9 & $a$ & $-1,8$ & $-1,9$ & -2 & $-1,90$ & -2 & -2 & -2 & $-2,00$ & $-0,6$ & $-0,6$ & $-0,7$ & $-0,63$ & $-0,7$ & $-0,7$ & $-0,7$ & $-0,70$ & 2,42 & $-0,03$ \\
\hline & b & 17,8 & 17,9 & 17,9 & 17,87 & 18 & 17,9 & 17,9 & 17,93 & 20 & 20 & 19,8 & 19,93 & 20 & 19,8 & 19,6 & 19,80 & & \\
\hline & L & 94,7 & 94,5 & 94,4 & 94,53 & 94,5 & 94,4 & 94,4 & 94,43 & 93 & 92,9 & 92,8 & 92,9 & 92,9 & 93 & 93,1 & 93,00 & & \\
\hline 10 & $\mathbf{a}$ & $-0,8$ & $-0,8$ & $-0,9$ & $-0,83$ & $-0,9$ & $-0,9$ & $-0,8$ & $-0,87$ & $\begin{array}{l}-0,3 \\
\end{array}$ & $-0,3$ & $\begin{array}{l}-0,3 \\
\end{array}$ & $-0,30$ & $-0,4$ & $-0,3$ & $-0,3$ & $\begin{array}{l}-0,33 \\
\end{array}$ & 1,72 & 0,03 \\
\hline & b & 21,3 & 21,2 & 21,1 & 21,20 & 21 & 21,5 & 21,8 & 21,43 & 21,3 & 21,2 & 21,4 & 21,30 & 21,3 & 21,7 & 21,7 & 21,57 & & \\
\hline
\end{tabular}


Quadro 26- Leituras de cor das amostras de E-max Press na espessura de 1,0mm cimentados com cimento autoadesivo.

\begin{tabular}{|c|c|c|c|c|c|c|c|c|c|c|c|c|c|c|c|c|c|c|c|}
\hline \multicolumn{20}{|c|}{ E-max Press 1,0mm Rely-X U200 } \\
\hline \multirow{2}{*}{$\begin{array}{c}\text { Corpo } \\
\text { de Prova }\end{array}$} & \multirow{2}{*}{ EIXO } & \multicolumn{4}{|c|}{ LEITURA INICIAL Branco } & \multicolumn{4}{|c|}{ LEITURA INICIAL Preto } & \multicolumn{4}{|c|}{ LEITURA Final Branco } & \multicolumn{4}{|c|}{ LEITURA FINAL Preto } & \multicolumn{2}{|c|}{$\Delta$} \\
\hline & & LI1 & LI2 & LI3 & MEDIA LI & LF1 & LF2 & LF3 & MEDIA LF & LI1 & $\mathrm{LI} 2$ & LI3 & MEDIA LI & LF1 & LF2 & LF3 & MEDIA LF & $\Delta \mathrm{E}$ & $\Delta \mathrm{T}$ \\
\hline \multirow{3}{*}{1} & $\mathbf{L}$ & 93,3 & 93,6 & 93,7 & 93,53 & 93,3 & 94,2 & 93,8 & 93,77 & 93,1 & 93,2 & 94,5 & 93,6 & 94,8 & 94,8 & 93,9 & 94,5 & \multirow{3}{*}{0,44} & \multirow{3}{*}{0,26} \\
\hline & a & $-0,8$ & $-0,7$ & $-0,8$ & $-0,77$ & $-0,8$ & $-0,8$ & $-0,8$ & $-0,80$ & $-0,8$ & $-0,8$ & $-0,9$ & $-0,83$ & $-0,9$ & $-0,9$ & $-0,9$ & $-0,9$ & & \\
\hline & b & 18,5 & 18,4 & 18,5 & 18,47 & 18,5 & 1,83 & 18,3 & 18,37 & 19 & 19 & 18,7 & 18,90 & 18,8 & 19 & 19,3 & 19,03 & & \\
\hline \multirow{3}{*}{2} & $\mathbf{L}$ & 94,1 & 94 & 94,5 & 94,20 & 94,9 & 94,4 & 94,5 & 94,60 & 93,3 & 93,7 & 93,4 & 93,47 & 93,8 & 94,2 & 93,9 & 93,97 & \multirow{3}{*}{1,06} & \multirow{3}{*}{0,40} \\
\hline & $\mathbf{a}$ & $-0,5$ & $-0,5$ & $-0,5$ & $-0,50$ & $-0,5$ & $-0,5$ & $-0,4$ & $-0,47$ & $-0,8$ & $-0,8$ & $-0,8$ & $-0,80$ & $-0,8$ & $-0,8$ & $-0,8$ & $-0,80$ & & \\
\hline & b & 20,1 & 20,2 & 20 & 20,10 & 19,9 & 20,1 & 20,1 & 20,07 & 19,2 & 19,3 & 19,7 & 19,40 & 19,5 & 19,7 & 19,6 & 19,60 & & \\
\hline \multirow{3}{*}{3} & $\mathbf{L}$ & 90 & 90,9 & 91,2 & 90,70 & 90,9 & 91 & 91,2 & 91,03 & 90,4 & 90,3 & 89,9 & 90,20 & 90 & 90,2 & 90,2 & 90,13 & \multirow{3}{*}{0,58} & \multirow{3}{*}{0,37} \\
\hline & $a$ & 0,1 & 0,1 & $-0,1$ & 0,03 & 0,2 & 0,1 & 0,1 & 0,13 & $-0,2$ & $-0,1$ & $-0,1$ & $-0,13$ & $-0,1$ & $-0,1$ & $-0,1$ & $-0,10$ & & \\
\hline & b & 21,1 & 21,2 & 21 & 21,1 & 21,4 & 21,2 & 21,1 & 21,23 & 20,6 & 20,7 & 21,3 & 20,87 & 20,9 & 20,9 & 20,9 & 20,90 & & \\
\hline \multirow{3}{*}{4} & L & 94,7 & 93,7 & 94 & 94,13 & 93,7 & 94 & 93,9 & 93,87 & 94,1 & 94 & 94 & 94,03 & 94,3 & 94,2 & 94,8 & 94,43 & \multirow{3}{*}{0,17} & \multirow{3}{*}{0,27} \\
\hline & $a$ & $-1,1$ & -1 & -1 & $-1,03$ & -1 & -1 & -1 & -1 & $-1,2$ & $-1,1$ & $-1,1$ & $-1,13$ & $-1,2$ & $-1,2$ & $-1,3$ & $-1,23$ & & \\
\hline & b & 17,8 & 17,8 & 17,7 & 17,77 & 17,8 & 17,7 & 17,9 & 17,80 & 17,8 & 17,9 & 17,9 & 17,87 & 18 & 18 & 17,8 & 17,93 & & \\
\hline \multirow{3}{*}{5} & L & 93,5 & 93,5 & 93,9 & 93,63 & 93,6 & 93,9 & 93,7 & 93,73 & 92,5 & 92,5 & 92,4 & 92,47 & 92,4 & 92,6 & 92,5 & 92,50 & \multirow{3}{*}{1,30} & \multirow{3}{*}{0,12} \\
\hline & $a$ & $-0,5$ & $-0,6$ & $-0,5$ & $-0,53$ & $-0,6$ & $-0,5$ & $-0,5$ & $-0,53$ & -08 & $-0,8$ & $-0,8$ & $-0,80$ & $-0,8$ & $-0,8$ & $-0,8$ & $-0,80$ & & \\
\hline & b & 20,6 & 20,7 & 20,8 & 20,70 & 20,4 & 20,9 & 20,6 & 20,63 & 20,1 & 20,3 & 20,2 & 20,20 & 20,2 & 20,2 & 20,1 & 20,17 & & \\
\hline 0 & $\mathbf{L}$ & 94,3 & 93,8 & 94,1 & 94,07 & 93,6 & 93,9 & 93,7 & 93,73 & 93,2 & 94,2 & 94,1 & 93,83 & 93,3 & 93,8 & 94,4 & 93,83 & & \\
\hline 6 & $\mathbf{a}$ & $-0,9$ & $-0,9$ & $-0,9$ & $-0,9$ & $-0,9$ & $-0,9$ & $-0,8$ & $-0,87$ & -1 & $-0,9$ & -1 & $-0,97$ & -1 & $-0,9$ & $-1,1$ & $-1,00$ & 0,28 & 0,40 \\
\hline & b & 18,5 & 18,2 & 18,4 & 18,37 & 18.1 & 18.2 & 18 & 18.1 & 18,3 & 18,2 & 18,2 & 18,23 & 18,1 & 18,3 & 18,4 & 18,27 & & \\
\hline & $\mathbf{L}$ & 93,7 & 93,6 & 93,5 & 93,60 & 93,6 & 93,9 & 93,5 & 93,67 & 93 & 93,1 & 93 & 93,03 & 93,4 & 92,8 & 93,2 & 93,13 & & \\
\hline 7 & $a$ & $-1,3$ & $-1,3$ & $-1,3$ & $-1,30$ & $-1,3$ & $-1,3$ & $-1,3$ & $-1,30$ & $-1,5$ & $-1,5$ & $-1,5$ & $-1,50$ & $-1,5$ & $-1,5$ & $-1,5$ & $-1,50$ & 0,64 & 0,12 \\
\hline & b & 17,9 & 17,8 & 17,8 & 17,83 & 17,7 & 17,7 & 17,8 & 17,73 & 18 & 18,1 & 18,1 & 18,07 & 18,1 & 18 & 17,8 & 17,97 & & \\
\hline & $\mathbf{L}$ & 93,6 & 93,6 & 94,2 & 93,80 & 95,1 & 93,8 & 93,8 & 94,23 & 93,5 & 93,7 & 93,9 & 93,70 & 93,9 & 94,1 & 94,4 & 94,13 & & \\
\hline 8 & $\mathbf{a}$ & $-0,7$ & $-0,7$ & $-0,7$ & $-0,70$ & $-0,7$ & $-0,7$ & $-0,7$ & $-0,70$ & $-0,9$ & $-0,8$ & $-0,8$ & $-0,83$ & $-0,8$ & $-0,8$ & $-0,8$ & $-0,80$ & 0,37 & 0,44 \\
\hline & b & 19,7 & 19,6 & 19,4 & 19,57 & 19,2 & 19,7 & 19,5 & 19,47 & 19,7 & 20 & 20 & 19,90 & 20,2 & 20,2 & 20,2 & 20,20 & & \\
\hline & $\mathbf{L}$ & 93,4 & 93,5 & 93,4 & 93,43 & 93,4 & 93,7 & 92,6 & 92,23 & 92,6 & 92,6 & 92,4 & 92,63 & 91,1 & 91,4 & 91,2 & 91,23 & & \\
\hline 9 & $a$ & $-0,8$ & $-0,8$ & $-0,8$ & $-0,80$ & $-0,8$ & $-0,8$ & $-0,8$ & $-0,80$ & -1 & $-1,1$ & -1 & $-1,03$ & $-0,9$ & $-0,9$ & $-0,9$ & $-0,90$ & 0,86 & 0,33 \\
\hline & b & 20,2 & 20,1 & 20,1 & 20,13 & 20,5 & 20,5 & 20,2 & 20,40 & 20,2 & 19,7 & 19,9 & 19,93 & 19,9 & 19,9 & 19,8 & 19,87 & & \\
\hline & $\mathbf{L}$ & 94,9 & 94,9 & 94,7 & 94,83 & 94,8 & 94,9 & 94,7 & 94,80 & 97,8 & 97,8 & 97,8 & 97,80 & 97,8 & 98,9 & 99 & 98,57 & & \\
\hline 10 & $\mathbf{a}$ & $-0,9$ & $-0,9$ & $-0,8$ & $-0,87$ & $-0,9$ & $-0,9$ & $-0,9$ & $-0,90$ & $-0,8$ & $\begin{array}{l}-0,8 \\
\end{array}$ & $\begin{array}{l}-0,8 \\
\end{array}$ & $-0,80$ & $-0,8$ & $-0,9$ & $\begin{array}{l}-0,9 \\
\end{array}$ & $-0,87$ & 3,37 & 0,05 \\
\hline & b & 19,2 & 19,4 & 19,1 & 19,23 & 19,3 & 19,2 & 19,2 & 19,23 & 20,8 & 20,9 & 20,8 & 20,83 & 20,9 & 20,5 & 20,5 & 20,63 & & \\
\hline
\end{tabular}


Quadro 27- Leituras de cor das amostras de ZirPress na espessura de 0,5mm cimentados com cimento fotoativado.

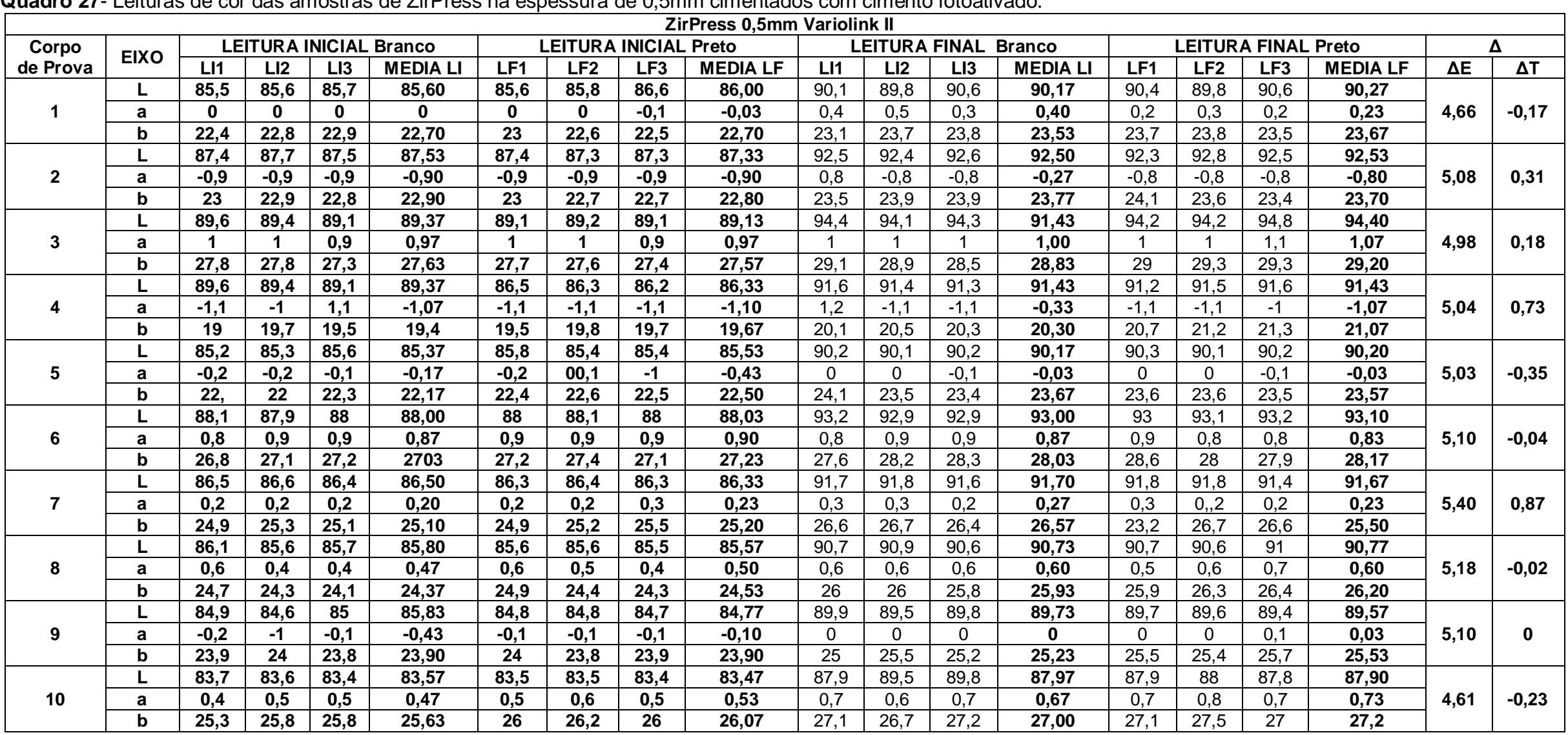


Quadro 28- Leituras de cor das amostras de ZirPress na espessura de 1,0mm cimentados com cimento fotoativado.

\begin{tabular}{|c|c|c|c|c|c|c|c|c|c|c|c|c|c|c|c|c|c|c|c|}
\hline \multicolumn{20}{|c|}{$\begin{array}{c}\text { ZirPress 1,0mm Variolink II } \\
\end{array}$} \\
\hline \multirow{2}{*}{$\begin{array}{c}\text { Corpo de } \\
\text { Prova }\end{array}$} & \multirow{2}{*}{ EIXO } & \multicolumn{4}{|c|}{ LEITURA INICIAL Branco } & \multicolumn{4}{|c|}{ LEITURA INICIAL Preto } & \multicolumn{4}{|c|}{ LEITURA Final Branco } & \multicolumn{4}{|c|}{ LEITURA FINAL Preto } & \multicolumn{2}{|c|}{$\Delta$} \\
\hline & & LI1 & LI2 & LI3 & MEDIA LI & LF1 & LF2 & LF3 & MEDIA LF & LI1 & LI2 & LI3 & MEDIA LI & LF1 & LF2 & LF3 & MEDIA LF & $\Delta \mathrm{E}$ & $\Delta T$ \\
\hline \multirow{3}{*}{1} & $\mathbf{L}$ & 90,6 & 91,3 & 91,2 & 91,03 & 90,6 & 91 & 91,2 & 90,93 & 90,8 & 90,1 & 90,7 & 90,53 & 90,8 & 90,8 & 91 & 90,87 & \multirow{3}{*}{0,72} & \multirow{3}{*}{$-0,18$} \\
\hline & $\mathbf{a}$ & $-0,7$ & $-0,9$ & $-0,8$ & $-0,80$ & $-0,4$ & $-0,5$ & $-0,3$ & $-0,40$ & $-0,7$ & $-0,6$ & $-0,7$ & $-0,67$ & $-0,7$ & $-0,7$ & $-0,8$ & $-0,73$ & & \\
\hline & b & 22,2 & 21,8 & 21,5 & 21,83 & 22,1 & 22 & 22,4 & 22,17 & 22,5 & 22 & 22,5 & 22,33 & 22,1 & 22,2 & 22,4 & 22,23 & & \\
\hline \multirow{3}{*}{2} & $L$ & 92,4 & 92,6 & 92,6 & 92,53 & 93 & 92,6 & 92,8 & 92,80 & 91,9 & 92,1 & 92,4 & 92,13 & 92,3 & 92,4 & 92,4 & 92,37 & \multirow{3}{*}{0,52} & \multirow{3}{*}{$-0,03$} \\
\hline & $\mathbf{a}$ & 0 & 0 & 0 & 0 & 0 & 0 & $-0,1$ & $-0,03$ & $-0,3$ & $-0,3$ & $-0,4$ & $-0,33$ & $-0,3$ & $-0,3$ & $-0,3$ & -030 & & \\
\hline & b & 25 & 25 & 25,2 & 25,07 & 25,1 & 25,1 & 24,9 & 25,03 & 25 & 25,2 & 25,2 & 25,13 & 25 & 25,3 & 25,3 & 25,20 & & \\
\hline \multirow{3}{*}{3} & $\mathbf{L}$ & 86,9 & 87,1 & 86,8 & 86,93 & 86,8 & 87,1 & 87 & 86,97 & 86,2 & 86,2 & 86,3 & 86,23 & 86 & 86,2 & 86,3 & 86,17 & \multirow{3}{*}{0,77} & \multirow{3}{*}{0,11} \\
\hline & a & $-0,4$ & $-0,5$ & $-0,4$ & $-0,43$ & $-0,5$ & $-0,5$ & $-0,5$ & $-0,50$ & $-0,7$ & $-0,6$ & $-0,7$ & $-0,67$ & $-0,6$ & $-0,6$ & $-0,6$ & $-0,60$ & & \\
\hline & b & 19,8 & 19,8 & 19,8 & 19,80 & 19,8 & 19,8 & 19,7 & 19,77 & 19,8 & 19,8 & 19,1 & 19,57 & 19,8 & 19,7 & 19,7 & 19,73 & & \\
\hline \multirow{3}{*}{4} & $\mathbf{L}$ & 94,6 & 95,2 & 94,6 & 94,80 & 94,7 & 94,7 & 94,7 & 94,70 & 92,3 & 92,4 & 92,5 & 92,40 & 92,7 & 92,7 & 92,7 & 92,70 & \multirow{3}{*}{2,72} & \multirow{3}{*}{0,23} \\
\hline & $a$ & $-1,1$ & $-1,1$ & -1 & $-1,07$ & $-1,1$ & $-1,1$ & $-1,1$ & $-1,10$ & $-1,5$ & $-1,5$ & $-1,5$ & $-1,50$ & $-1,4$ & $-1,4$ & $-1,4$ & $-1,40$ & & \\
\hline & b & 22 & 22,1 & 22,1 & 22,07 & 22 & 22,1 & 22,2 & 22,10 & 20,7 & 20,9 & 21 & 20,87 & 20,7 & 20,7 & 20,8 & 20,73 & & \\
\hline \multirow{3}{*}{5} & $\mathbf{L}$ & 97,4 & 97,9 & 97,4 & 97,57 & 97,3 & 97,3 & 97,2 & 97,27 & 96 & 96 & 96,1 & 96,03 & 96,1 & 96,1 & 96,1 & 96,10 & \multirow{3}{*}{1,70} & \multirow{3}{*}{0,03} \\
\hline & $a$ & -09 & $-0,9$ & $-0,9$ & $-0,90$ & $-0,9$ & $-0,9$ & $-0,9$ & $-0,90$ & $-1,1$ & $-1,1$ & $-1,1$ & $-1,10$ & $-1,2$ & $-1,2$ & $-1,1$ & $-1,17$ & & \\
\hline & b & 22,8 & 22,7 & 22,8 & 22,77 & 22,5 & 22,6 & 22,9 & 22,67 & 21,9 & 22,1 & 22,2 & 22,07 & 22,3 & 22,4 & 22,5 & 22,40 & & \\
\hline & $\mathbf{L}$ & 92,6 & 92,6 & 92,8 & 92,67 & 92,7 & 92,7 & 92,9 & 92,77 & 91,9 & 92 & 91,9 & 91,93 & 91,9 & 92 & 92 & 91,97 & & \\
\hline 6 & $a$ & $-0,9$ & $-0,9$ & $-0,9$ & $-0,90$ & $-0,9$ & $-0,9$ & $-0,9$ & $-0,90$ & $-1,1$ & $-1,1$ & $-1,1$ & $-1,10$ & $-1,1$ & $-1,1$ & $-1,1$ & $-1,10$ & 0,80 & $-0,05$ \\
\hline & b & 22 & 21,9 & 22 & 21,97 & 22 & 21,9 & 22 & 21,97 & 22,2 & 22,2 & 22,2 & 22,20 & 22,2 & 22,2 & 22,3 & 22,23 & & \\
\hline & $\mathbf{L}$ & 97,3 & 96,9 & 97,5 & 97,26 & 97,4 & 97,2 & 96,9 & 91,17 & 94,7 & 95 & 95,2 & 94,97 & 94,7 & 94,3 & 94,6 & 94,53 & & \\
\hline 7 & $\mathbf{a}$ & $-1,2$ & $-1,2$ & $-1,2$ & $-1,20$ & $-1,1$ & $-1,1$ & $-1,1$ & $-1,10$ & $-1,8$ & $-1,8$ & $-1,8$ & $-1,80$ & $-1,8$ & $-1,7$ & $-1,7$ & $-1,73$ & 2,65 & 0,13 \\
\hline & b & 21 & 21,2 & 20,9 & 21,03 & 21,1 & 21,3 & 21,6 & 21,33 & 19,7 & 19,8 & 19,9 & 19,80 & 20 & 19,9 & 19,8 & 19,90 & & \\
\hline & $L$ & 91,4 & 91,3 & 91,5 & 91,40 & 91,8 & 91 & 91 & 91,27 & 89,1 & 89,3 & 89 & 89,13 & 89,4 & 88,8 & 89 & 89,07 & & \\
\hline 8 & $\mathbf{a}$ & 0 & 0 & $-0,1$ & $-0,03$ & $-0,2$ & $-0,2$ & $-0,2$ & $-0,20$ & $-0,9$ & $-0,9$ & $-0,9$ & $-0,90$ & -1 & $-0,9$ & -1 & -097 & 2,79 & $-0,08$ \\
\hline & b & 24,9 & 24,9 & 25,1 & 24,97 & 25,2 & 24,9 & 24,7 & 24,93 & 23,4 & 23,7 & 23,7 & 23,60 & 23,6 & 23,3 & 23,6 & 23,50 & & \\
\hline & L & 95,1 & 95,1 & 95,2 & 95,13 & 95,1 & 95 & 95,6 & 95,23 & 93,6 & 93,5 & 93,8 & 93,63 & 93,7 & 93,8 & 93,6 & 93,70 & & \\
\hline 9 & $\mathbf{a}$ & $-1,3$ & $-1,3$ & $\begin{array}{l}-1,3 \\
\end{array}$ & $-1,30$ & $-1,3$ & $\begin{array}{l}-1,3 \\
\end{array}$ & $-1,3$ & $-1,30$ & $-1,5$ & $-1,4$ & $-1,5$ & $-1,47$ & $-1,4$ & $-1,4$ & $\begin{array}{l}-1,4 \\
\end{array}$ & $\begin{array}{l}-1,40 \\
\end{array}$ & 1,57 & $-0,01$ \\
\hline & b & 20,1 & 20,5 & 20,5 & 20,37 & 20,4 & 20,2 & 20,5 & 20,37 & 20 & 19,8 & 20 & 19,93 & 20 & 19,9 & 19,9 & 19,93 & & \\
\hline & $L$ & 93,2 & 92,5 & 92,1 & 92,60 & 93,1 & 92,3 & 92,4 & 92,60 & 91,6 & 1,5 & 91,3 & 91,47 & 91,4 & 91,2 & 91,8 & 91,47 & & \\
\hline 10 & $\mathbf{a}$ & $-1,5$ & $-1,4$ & $-1,5$ & $-1,47$ & $-1,3$ & $-1,5$ & $-1,5$ & $-1,43$ & $-1,7$ & $-1,8$ & $-1,8$ & $-1,77$ & $-1,9$ & $-1,8$ & $-1,8$ & $-1,83$ & 1,37 & $-0,01$ \\
\hline & b & 19,2 & 18,9 & 19,9 & 19,07 & 19,3 & 18,9 & 18,8 & 19,00 & 18,4 & 18,4 & 18,3 & 18,37 & 18,1 & 18,3 & 18,7 & 18,37 & & \\
\hline
\end{tabular}


Quadro 29- Leituras de cor das amostras de ZirPress na espessura de 0,5mm cimentados com cimento autoadesivo.

\begin{tabular}{|c|c|c|c|c|c|c|c|c|c|c|c|c|c|c|c|c|c|c|c|}
\hline \multicolumn{20}{|c|}{ ZirPress $0,5 \mathrm{~mm}$ Rely-X U200 } \\
\hline \multirow{2}{*}{$\begin{array}{l}\text { Corpo de } \\
\text { Prova }\end{array}$} & \multirow{2}{*}{ EIXO } & \multicolumn{4}{|c|}{ LEITURA INICIAL Branco } & \multicolumn{4}{|c|}{ LEITURA INICIAL Preto } & \multicolumn{4}{|c|}{ LEITURA Final Branco } & \multicolumn{4}{|c|}{ LEITURA FINAL Preto } & \multicolumn{2}{|c|}{$\Delta$} \\
\hline & & LI1 & $\mathrm{LI} 2$ & LI3 & MEDIA LI & LF1 & LF2 & LF3 & MEDIA LF & LI1 & LI2 & LI3 & MEDIA LI & LF1 & LF2 & LF3 & MEDIA LF & $\Delta \mathrm{E}$ & $\Delta \mathrm{T}$ \\
\hline \multirow{3}{*}{1} & $\mathbf{L}$ & 92 & 92,5 & 93,3 & 92,60 & 92,6 & 92,4 & 92,7 & 92,57 & 91,3 & 90,5 & 91 & 90,93 & 91,1 & 90,9 & 91 & 91,00 & \multirow{3}{*}{1,76} & \multirow{3}{*}{0,03} \\
\hline & $\mathbf{a}$ & $-0,4$ & $-0,5$ & $-0,6$ & $-0,50$ & $-0,5$ & $-0,5$ & $-0,5$ & $-0,50$ & $-0,1$ & 0 & $-0,1$ & $-0,07$ & $-0,1$ & $-0,2$ & $-0,2$ & $-0,17$ & & \\
\hline & b & 26,8 & 27 & 27,2 & 27,00 & 27,2 & 27,1 & 27,3 & 27,20 & 26,6 & 26,7 & 26,6 & 26,63 & 26,8 & 27 & 26,7 & 26,83 & & \\
\hline \multirow{3}{*}{2} & $\mathbf{L}$ & 92,9 & 92,7 & 92,5 & 92,70 & 92,8 & 92,5 & 92,4 & 92,57 & 91,7 & 91,6 & 91,6 & 91,63 & 92 & 92,1 & 91,4 & 91,83 & \multirow{3}{*}{1,11} & \multirow{3}{*}{$-0,01$} \\
\hline & $\mathbf{a}$ & $-1,6$ & $-1,4$ & $-1,4$ & $-1,47$ & $-1,3$ & $-1,3$ & $-1,3$ & $-1,30$ & $-1,4$ & $-1,4$ & $\begin{array}{l}-1,4 \\
\end{array}$ & $-1,40$ & $\begin{array}{l}-1,3 \\
\end{array}$ & $\begin{array}{l}-1,4 \\
\end{array}$ & $\begin{array}{l}-1,4 \\
\end{array}$ & $-1,37$ & & \\
\hline & b & 22,3 & 22,2 & 22,2 & 22,23 & 22,3 & 22,4 & 22,3 & 22,33 & 22,5 & 22,5 & 22,6 & 22,53 & 22,5 & 22,7 & 22,7 & 22,63 & & \\
\hline \multirow{3}{*}{3} & $\mathbf{L}$ & 93,8 & 94,3 & 94,5 & 94,20 & 94,1 & 92,5 & 92,4 & 92,57 & 92,9 & 92,5 & 92,6 & 92,67 & 92,6 & 92,4 & 92,5 & 92,50 & \multirow{3}{*}{1,54} & \multirow{3}{*}{0,39} \\
\hline & $\mathbf{a}$ & $-0,7$ & $-0,8$ & $-0,9$ & $-0,80$ & $-0,8$ & $-0,8$ & $-0,8$ & $-0,80$ & $-0,9$ & $\begin{array}{l}-0,8 \\
\end{array}$ & $-0,9$ & $-0,87$ & $-0,9$ & $-0,9$ & $-0,9$ & $-0,90$ & & \\
\hline & b & 23,9 & 24,3 & 23,9 & 24,03 & 24,1 & 23,6 & 23,9 & 23,87 & 24 & 24,3 & 24,1 & 24,13 & 23,2 & 23,6 & 23,7 & 23,53 & & \\
\hline \multirow{3}{*}{4} & L & 86,6 & 86,5 & 86,4 & 86,50 & 86,4 & 86,4 & 86,8 & 86,53 & 86 & 86 & 85,8 & 85,93 & 85,8 & 85,5 & 86 & 85,77 & \multirow{3}{*}{0,58} & \multirow{3}{*}{0,12} \\
\hline & $a$ & $-0,8$ & $-0,8$ & $-0,7$ & $-0,77$ & $-0,7$ & $-0,7$ & $-0,8$ & $-0,73$ & $-0,8$ & $-0,6$ & $-0,7$ & $-0,70$ & $-0,7$ & $-0,7$ & $\begin{array}{l}-0,8 \\
\end{array}$ & $-0,73$ & & \\
\hline & b & 25,5 & 25,1 & 25,6 & 25,40 & 25,6 & 25,4 & 25,3 & 25,43 & 25,4 & 25,5 & 25 & 25,3 & 25,5 & 25,3 & 25,2 & 25,33 & & \\
\hline \multirow{3}{*}{5} & $\mathbf{L}$ & 88,7 & 88,7 & 88,7 & 88,70 & 88,4 & 88,9 & 87,9 & 88,4 & 88,2 & 88,5 & 87,2 & 87,97 & 87,7 & 87,8 & 87,6 & 87,70 & \multirow{3}{*}{0,95} & \multirow{3}{*}{0,08} \\
\hline & $a$ & $-1,8$ & $-1,8$ & $-1,8$ & $-1,80$ & $-1,8$ & $-1,8$ & $-1,7$ & $-1,77$ & $-1,9$ & $-1,9$ & $-1,8$ & $-1,87$ & $-1,8$ & $-1,8$ & $-1,9$ & $-1,83$ & & \\
\hline & b & 15,4 & 15,6 & 15,6 & 15,53 & 15,7 & 15,5 & 15,7 & 15,63 & 16,1 & 16,4 & 15,9 & 16,13 & 15,8 & 15,8 & 15,9 & 15,83 & & \\
\hline & $\mathbf{L}$ & 93,3 & 93,4 & 93,2 & 93,30 & 93,2 & 93,3 & 93,5 & 93,33 & 91,5 & 91,8 & 91,7 & 91,67 & 91,6 & 91,8 & 92 & 91,8 & & \\
\hline 6 & $\mathbf{a}$ & $-0,4$ & $-0,4$ & $-0,3$ & $-0,37$ & $-0,3$ & $-0,4$ & $-0,5$ & $-0,4$ & $-0,5$ & $-0,5$ & $-0,4$ & $-0,47$ & $-0,4$ & $-0,5$ & $-0,6$ & $-0,50$ & 1,65 & 0,01 \\
\hline & b & 25,3 & 25,4 & 25,7 & 25,47 & 25,6 & 25,8 & 25,4 & 25,60 & 25,1 & 25,4 & 25,3 & 25,27 & 25 & 25,2 & 25,4 & 25,20 & & \\
\hline & $\mathbf{L}$ & 91,9 & 91,8 & 91,5 & 91,73 & 91,3 & 91,2 & 91,3 & 91,27 & 91 & 90,4 & 90,4 & 90,60 & 90,6 & 89,9 & 90,4 & 90,30 & & \\
\hline 7 & $\mathbf{a}$ & $-1,7$ & $-1,7$ & $-1,7$ & $-1,7$ & $-1,7$ & $-1,8$ & $-1,7$ & $-1,73$ & $-1,6$ & $-1,8$ & $-1,8$ & $-1,73$ & $-1,9$ & $-1,8$ & $-1,8$ & $-1,83$ & 1,16 & $-0,06$ \\
\hline & b & 17,5 & 17,8 & 17,7 & 17,67 & 17,5 & 17,4 & 17,6 & 17,50 & 18 & 17,8 & 179 & 17,90 & 17,6 & 17,5 & 17,7 & 17,6 & & \\
\hline & $\mathbf{L}$ & 93,9 & 94 & 941 & 94 & 94 & 93,6 & 94 & 93,87 & 93,8 & 93,6 & 93,5 & 93,63 & 93,5 & 93,8 & 93,8 & 93,7 & & \\
\hline 8 & $a$ & 0,2 & 0,2 & 0,2 & 0,20 & 0,2 & 0,2 & 0,2 & 0,2 & 0,5 & 0,5 & 0,6 & 0,53 & 0,6 & 0,5 & 0,5 & 0,53 & 0,18 & $-0,04$ \\
\hline & b & 29,6 & 29,8 & 29,9 & 29,77 & 30,1 & 29,7 & 29,6 & 29,8 & 30,5 & 30,2 & 30,5 & 30,4 & 30,5 & 30,2 & 30,3 & 30,33 & & \\
\hline & $\mathbf{L}$ & 92,3 & 92,3 & 92,2 & 92,27 & 92,4 & 92,3 & 92,4 & 92,37 & 91,2 & 91,2 & 91,2 & 91,20 & 91,2 & 91,2 & 91 & 91,13 & & \\
\hline 9 & $a$ & -2 & -2 & -2 & -2 & $-1,9$ & -2 & $-2,1$ & -2 & -0 & $-1,9$ & $-1,8$ & $-1,90$ & $-1,8$ & -2 & $-1,9$ & 1,90 & 1,17 & $-0,05$ \\
\hline & b & 16,2 & 16,4 & 16,4 & 16,33 & 16,7 & 16,3 & 16,4 & 16,47 & 16,9 & 16,6 & 16,9 & 16,80 & 17 & 16,5 & 16,6 & 16,70 & & \\
\hline & $\mathbf{L}$ & 93,6 & 93,5 & 93,5 & 93,53 & 93,5 & 93,4 & 93,7 & 93,53 & 92,9 & 92,5 & 92,8 & 92,73 & 92,4 & 92,6 & 92,7 & 92,57 & & \\
\hline 10 & $a$ & $-0,8$ & $-0,8$ & -1 & $-0,87$ & -1 & -1 & -1 & $-1,00$ & $-0,9$ & $-0,7$ & $-0,8$ & $-0,8$ & $-0,7$ & $-0,8$ & $-0,6$ & $-0,7$ & 0,82 & 0,09 \\
\hline & b & 26,2 & 26,4 & 25,9 & 26,17 & 26,1 & 26 & 26,1 & 26,07 & 25,5 & 26,6 & 26,9 & 26,33 & 26,5 & 26,2 & 26,8 & 26,50 & & \\
\hline
\end{tabular}


Quadro 30- Leituras de cor das amostras de ZirPress na espessura de 1,0mm cimentados com cimento autoadesivo.

\begin{tabular}{|c|c|c|c|c|c|c|c|c|c|c|c|c|c|c|c|c|c|c|c|}
\hline \multicolumn{20}{|c|}{ E-max Press 1,0mm Rely-X U200 } \\
\hline \multirow{2}{*}{$\begin{array}{c}\text { Corpo de } \\
\text { Prova }\end{array}$} & \multirow[b]{2}{*}{ EIXO } & \multicolumn{4}{|c|}{ LEITURA INICIAL Branco } & \multicolumn{4}{|c|}{ LEITURA INICIAL Preto } & \multicolumn{4}{|c|}{ LEITURA Final Branco } & \multicolumn{4}{|c|}{ LEITURA FINAL Preto } & \multicolumn{2}{|c|}{$\Delta$} \\
\hline & & LI1 & LI2 & LI3 & MEDIA LI & LF1 & LF2 & LF3 & MEDIA LF & LI1 & LI2 & LI3 & MEDIA LI & LF1 & LF2 & LF3 & MEDIA LF & $\Delta \mathrm{E}$ & $\Delta T$ \\
\hline \multirow{3}{*}{1} & $\mathbf{L}$ & 91,2 & 91,6 & 91,9 & 91,57 & 91,7 & 91,7 & 91,7 & 91,70 & 91,6 & 92,2 & 92,7 & 92,17 & 91,3 & 92,1 & 92,8 & 92,07 & \multirow{3}{*}{1,32} & \multirow{3}{*}{0,17} \\
\hline & $\mathbf{a}$ & $-1,7$ & $-1,7$ & $-1,7$ & $-1,70$ & $-1,6$ & $-1,6$ & $-1,6$ & $-1,60$ & $-1,1$ & $-1,1$ & $-1,2$ & $-1,13$ & $-0,9$ & $-1,1$ & $-1,2$ & $-1,07$ & & \\
\hline & b & 18,9 & 19 & 19 & 18,97 & 19 & 18,9 & 18,9 & 18,93 & 19,9 & 20,2 & 19,9 & 20,00 & 20,5 & 20,2 & 20,1 & 20,27 & & \\
\hline \multirow{3}{*}{2} & $\mathbf{L}$ & 92,4 & 93,3 & 92,7 & 92,80 & 92,5 & 92,5 & 92,5 & 92,50 & 90,4 & 90,4 & 90,5 & 90,43 & 90,8 & 90,8 & 90,8 & 90,80 & \multirow{3}{*}{2,44} & \multirow{3}{*}{0,34} \\
\hline & $a$ & -2 & $-1,9$ & $-1,9$ & $-1,93$ & $-2,2$ & -2 & $-2,1$ & $-2,10$ & $-1,7$ & $-1,6$ & $-1,6$ & $-1,63$ & $-1,7$ & $-1,6$ & $-1,7$ & $-1,67$ & & \\
\hline & b & 17 & 17,4 & 17,3 & 17,23 & 17,3 & 17,3 & 17,1 & 17,23 & 16,6 & 16,8 & 16,8 & 16,73 & 17 & 16,9 & 17 & 16,97 & & \\
\hline \multirow{3}{*}{3} & $\mathbf{L}$ & 92,4 & 92,6 & 92,7 & 92,57 & 92,7 & 92,7 & 92,8 & 92,73 & 91 & 91,2 & 91,3 & 91,17 & 91,2 & 91,5 & 91,3 & 91,33 & \multirow{3}{*}{1,42} & \multirow{3}{*}{0,19} \\
\hline & $a$ & -2 & -2 & -2 & $-2,00$ & $-2,1$ & $-2,1$ & $-2,1$ & $-2,10$ & $-1,8$ & $-1,8$ & $-1,8$ & $-1,80$ & $-1,8$ & $-1,8$ & $-1,8$ & $-1,80$ & & \\
\hline & b & 18,2 & 18,3 & 18,6 & 18,37 & 18,2 & 18,4 & 18,5 & 18,37 & 18,1 & 18,3 & 18,3 & 18,23 & 18,5 & 18,1 & 18,3 & 18,30 & & \\
\hline \multirow{3}{*}{4} & $\mathbf{L}$ & 90,7 & 91,2 & 90,9 & 90,93 & 90,9 & 91,6 & 91,1 & 91,20 & 89,8 & 89,9 & 90,1 & 89,93 & 90,1 & 90,1 & 90,2 & 90,13 & \multirow{3}{*}{1,04} & \multirow{3}{*}{0,33} \\
\hline & $a$ & $-1,7$ & $-1,6$ & $-1,6$ & \begin{tabular}{|l|}
$-1,63$ \\
\end{tabular} & $-1,7$ & $\begin{array}{l}-1,6 \\
\end{array}$ & $-1,6$ & $\begin{array}{l}-1,63 \\
\end{array}$ & $\begin{array}{l}-1,3 \\
\end{array}$ & $\begin{array}{l}-1,4 \\
\end{array}$ & $\begin{array}{l}-1,4 \\
\end{array}$ & $\begin{array}{l}-1,37 \\
\end{array}$ & $-1,4$ & $\begin{array}{l}-1,4 \\
\end{array}$ & $\begin{array}{l}-1,4 \\
\end{array}$ & $\begin{array}{l}-1,40 \\
\end{array}$ & & \\
\hline & b & 18,3 & 18,6 & 18,5 & 18,47 & 18,5 & 18,9 & 18,6 & 18,67 & 18,4 & 18,6 & 18,6 & 18,53 & 18,5 & 18,6 & 18,5 & 18,53 & & \\
\hline \multirow{3}{*}{5} & $\mathbf{L}$ & 95,3 & 95,2 & 95,2 & 95,23 & 95,1 & 95,1 & 95,2 & 95,13 & 93,5 & 93,6 & 93,5 & 93,53 & 93,5 & 93,6 & 93,5 & 93,53 & \multirow{3}{*}{2,01} & \multirow{3}{*}{0,11} \\
\hline & $\mathbf{a}$ & $-1,2$ & $-1,3$ & $-1,3$ & $-1,27$ & $-1,3$ & $-1,3$ & $-1,3$ & $-1,30$ & $\begin{array}{l}-1,3 \\
\end{array}$ & $-1,3$ & $\begin{array}{l}-1,3 \\
\end{array}$ & $-1,30$ & $\begin{array}{l}-1,3 \\
\end{array}$ & $-1,3$ & $\begin{array}{l}-1,3 \\
\end{array}$ & $-1,30$ & & \\
\hline & b & 22,6 & 22 & 22 & 22,2 & 22,1 & 22,2 & 22,3 & 22,20 & 21 & 21,2 & 21,2 & 21,13 & 21,1 & 21 & 21 & 21,03 & & \\
\hline & $\mathbf{L}$ & 95,4 & 94,6 & 95,1 & 95,03 & 94,7 & 94,8 & 94,4 & 94,63 & 90,1 & 89,9 & 90 & 90,00 & 90,2 & 90,4 & 90,4 & 90,33 & & \\
\hline 6 & $a$ & $-0,8$ & $-0,8$ & $-0,8$ & $-0,80$ & $-0,8$ & $-0,8$ & $-0,9$ & $\begin{array}{l}-0,83 \\
\end{array}$ & $\begin{array}{l}-1,2 \\
\end{array}$ & $\begin{array}{l}-1,2 \\
\end{array}$ & $-1,2$ & $-1,20$ & $\begin{array}{l}-1,2 \\
\end{array}$ & $\begin{array}{l}-1,2 \\
\end{array}$ & $\begin{array}{l}-1,2 \\
\end{array}$ & $-1,20$ & 5,84 & 0,41 \\
\hline & b & 22,7 & 22,9 & 22,8 & 22,80 & 22,9 & 22,8 & 22,9 & 22,87 & 19,6 & 20,1 & 19,9 & 19,87 & 19,9 & 20 & 20 & 19,97 & & \\
\hline & $\mathbf{L}$ & 94,9 & 94,7 & 95,1 & 95,03 & 94,8 & 95 & 94,6 & 94,80 & 90,8 & 90,7 & 90,7 & 90,73 & 90,9 & 90,9 & 90,9 & 90,90 & & \\
\hline 7 & $\mathbf{a}$ & $-0,4$ & $-0,4$ & $-0,3$ & $-0,37$ & $-0,4$ & $-0,4$ & $-0,4$ & $-0,40$ & $-0,6$ & $-0,5$ & $-0,5$ & $-0,53$ & $-0,5$ & $-0,5$ & $-0,6$ & $-0,53$ & 4,51 & 0,07 \\
\hline & b & 24,7 & 24,7 & 24,6 & 24,67 & 24,7 & 24,7 & 24,6 & 24,67 & 22,5 & 22,7 & 22,6 & 22,60 & 22,7 & 22,6 & 22,4 & 22,57 & & \\
\hline & $\mathbf{L}$ & 94,2 & 94,3 & 94,3 & 94,27 & 94,6 & 94,4 & 94,8 & 94,60 & 91,7 & 91,7 & 92 & 91,80 & 91,8 & 92,4 & 92,1 & 92,10 & & \\
\hline 8 & $a$ & -1 & -1 & -1 & $-1,00$ & -1 & -1 & -1 & $-1,00$ & $-1,1$ & $-1,1$ & $-1,1$ & $-1,10$ & -1 & $-1,1$ & $-1,1$ & $-1,07$ & & 0,35 \\
\hline & b & 22,8 & 22,9 & 22,9 & 22,87 & 22,9 & 23 & 23 & 22,97 & 21,4 & 21,5 & 21,6 & 21,50 & 21,5 & 21,7 & 21,6 & 21,60 & & \\
\hline & $\mathbf{L}$ & 94,4 & 94,3 & 94,6 & 94,43 & 94,6 & 94,6 & 94,6 & 94,60 & 90,8 & 91 & 91,2 & 91,00 & 91,1 & 91,2 & 91,3 & 91,20 & & \\
\hline 9 & $a$ & $-0,7$ & $-0,7$ & $-0,7$ & $-0,70$ & $-0,8$ & $-0,7$ & $-0,8$ & $-0,77$ & -1 & -1 & $-1,1$ & \begin{tabular}{|l|}
$-1,03$ \\
\end{tabular} & -1 & -1 & -1 & $-1,00$ & & 0,18 \\
\hline & b & 23,4 & 23,4 & 23,6 & 23,47 & 23,4 & 23,6 & 23,4 & 23,47 & 20,9 & 21,2 & 20,8 & 20,97 & 21 & 21 & 21,1 & 21,03 & & \\
\hline & $\mathbf{L}$ & 96,1 & 95,9 & 95,9 & 95,97 & 96 & 95,6 & 95,8 & 95,80 & 92,1 & 92 & 92 & 92,03 & 92,1 & 92,2 & 92,1 & 92,13 & & \\
\hline 10 & $a$ & -1 & -1 & $-1,1$ & $\begin{array}{l}-1,03 \\
\end{array}$ & -1 & -1 & 1,1 & $\begin{array}{l}-1,03 \\
\end{array}$ & $\begin{array}{l}-1,2 \\
\end{array}$ & $\begin{array}{l}-1,2 \\
\end{array}$ & $\begin{array}{l}-1,2 \\
\end{array}$ & $-1,20$ & $\begin{array}{l}-1,2 \\
\end{array}$ & $\begin{array}{l}-1,2 \\
\end{array}$ & $\begin{array}{l}-1,2 \\
\end{array}$ & $\begin{array}{l}-1,20 \\
\end{array}$ & & 0,26 \\
\hline & b & 22,3 & 22,3 & 22,2 & 22,27 & 22,1 & 22,1 & 22 & 22,07 & 21,3 & 21,1 & 21,3 & 21,23 & 21,4 & 21,5 & 21,3 & 21,40 & & \\
\hline
\end{tabular}


Quadro 31- Leituras de cor das amostras do Cimento fotoativado (grupo controle).

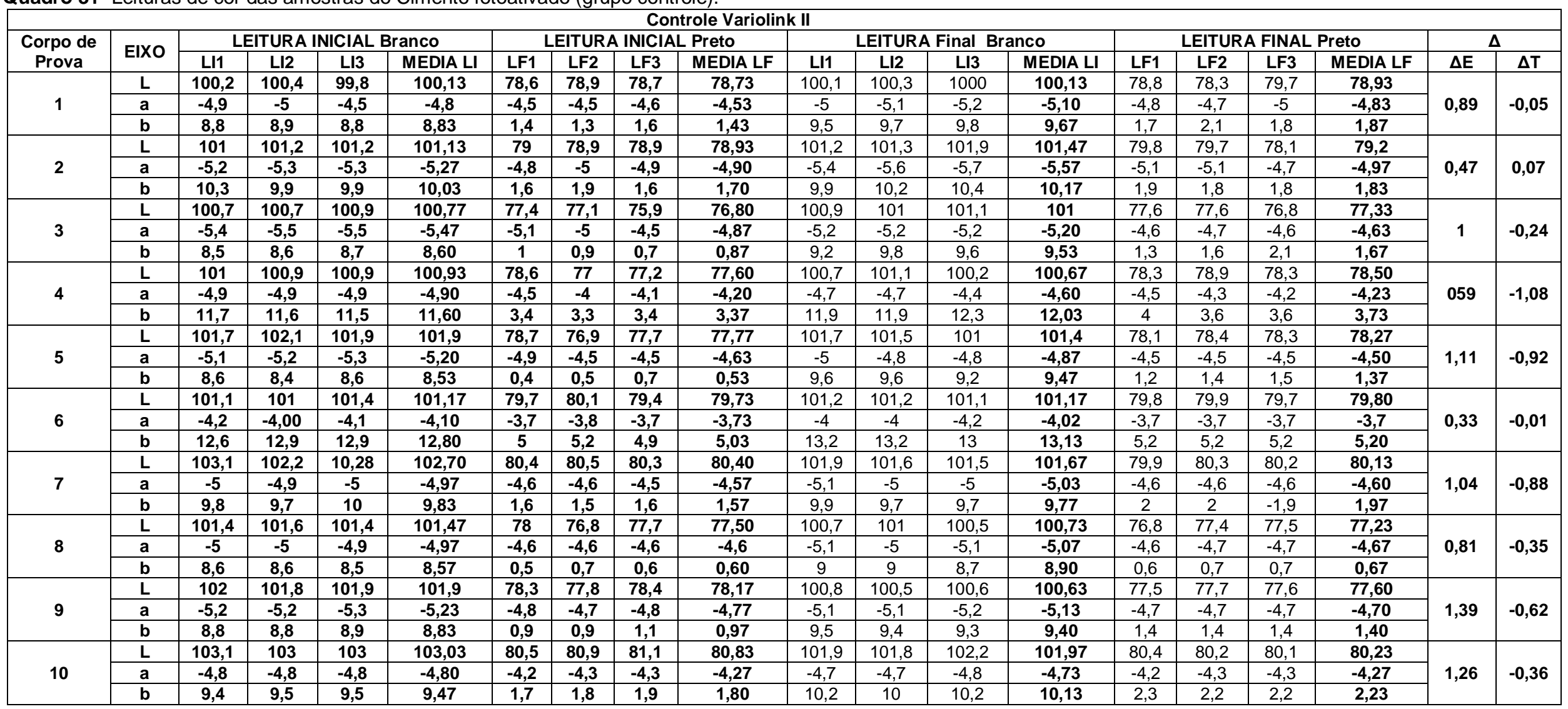


Quadro 32- Leituras de cor das amostras do Cimento Autoadesivo (grupo controle).

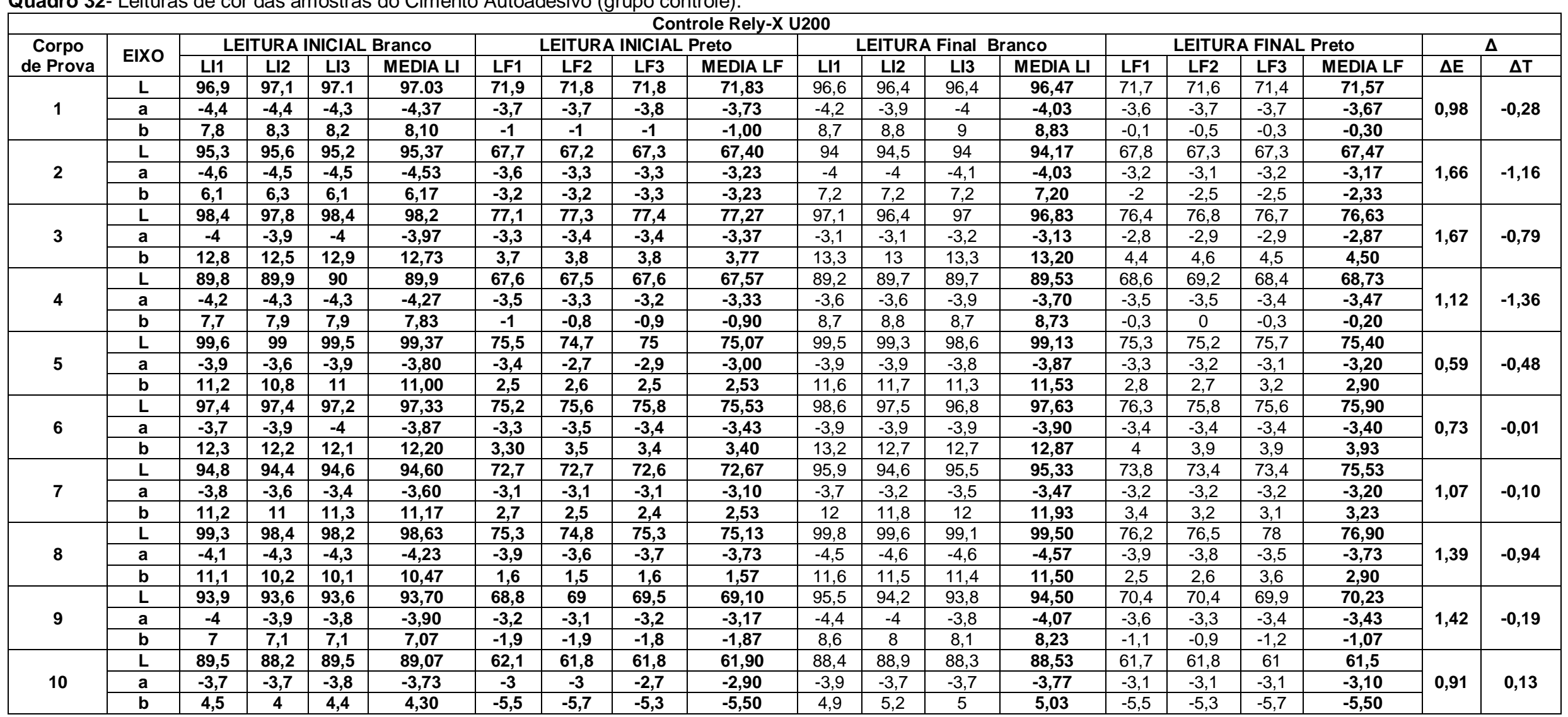


Quadro 33- Leituras de cor das amostras dos Dentes (grupo controle).

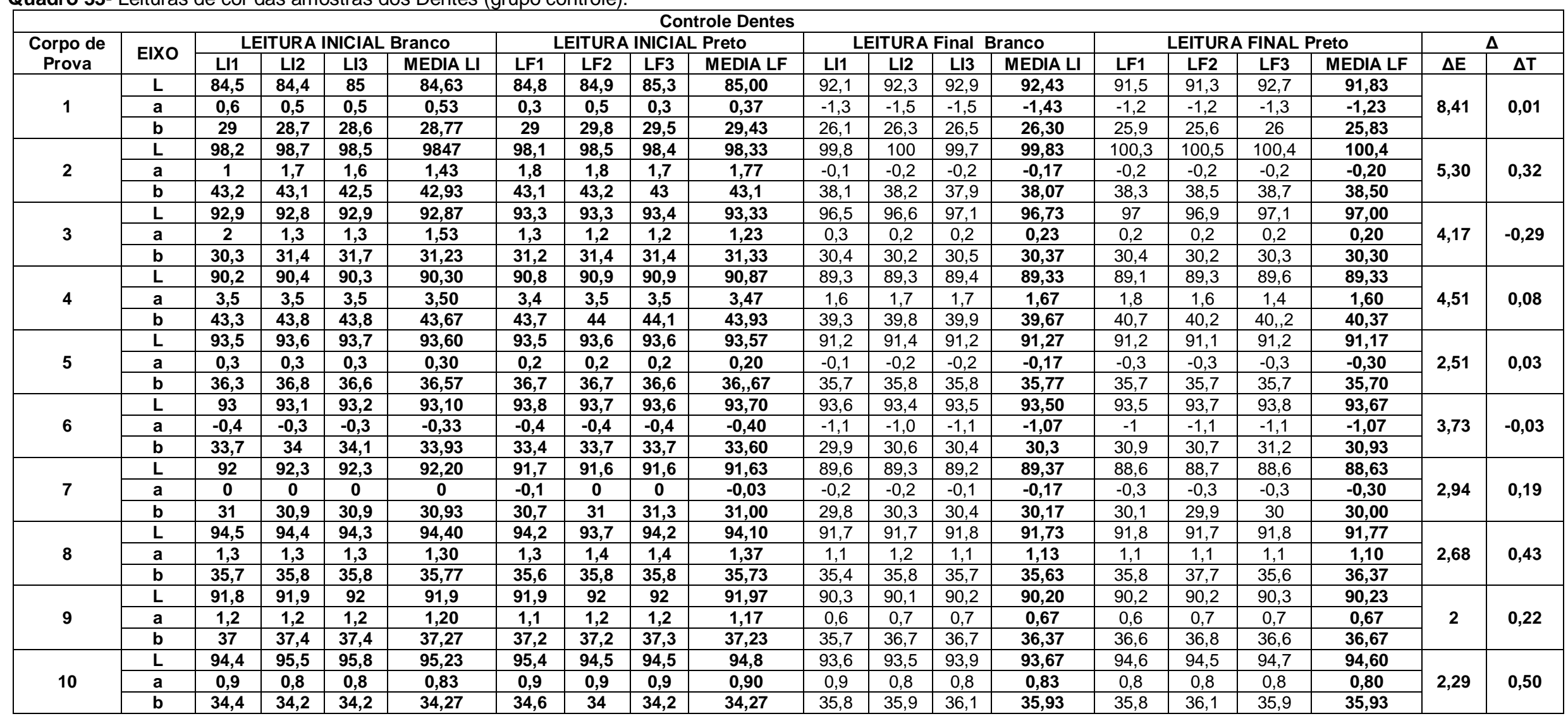


O quadro 34 apresenta os valores em triplicata das médias da análise da linha de cimentação dos grupos submetidos a agua destilada.

\begin{tabular}{|c|c|c|c|c|c|c|c|c|c|c|c|c|c|}
\hline \multirow{2}{*}{ Grupo } & \multirow{2}{*}{$\begin{array}{c}\text { Corpo de } \\
\text { prova }\end{array}$} & \multicolumn{4}{|c|}{ Ciclagem Termomecânica } & \multicolumn{4}{|c|}{ Envelhecimento em água } & \multicolumn{4}{|c|}{ Controle } \\
\hline & & Leitura 1 & Leitura 2 & Leitura 3 & Média & Leitura 1 & Leitura 2 & Leitura 3 & Média & Leitura 1 & Leitura 2 & Leitura 3 & Média \\
\hline \multirow{3}{*}{$\begin{array}{c}\text { E.max } \\
0.5 \mathrm{~V}\end{array}$} & 1 & 0,060 & 0,075 & 0,078 & 0,071 & 0,044 & 0,081 & 0,056 & 0,060 & 0,043 & 0,046 & 0,041 & 0,043 \\
\hline & 2 & 0,095 & 0,061 & 0,089 & 0,072 & 0,129 & 0,091 & 0,080 & 0,100 & 0,104 & 0,050 & 0,023 & 0,027 \\
\hline & 3 & 0,068 & 0,068 & 0,080 & 0,072 & 0,061 & 0,066 & 0,041 & 0,056 & 0,061 & 0,024 & 0,028 & 0,037 \\
\hline \multirow{3}{*}{$\begin{array}{c}\text { E.max } \\
0.5 \mathrm{R}\end{array}$} & 1 & 0,020 & 0,024 & 0,029 & 0,024 & 0,051 & 0,041 & 0,034 & 0,042 & 0,068 & 0,070 & 0,037 & 0,058 \\
\hline & 2 & 0,031 & 0,052 & 0,100 & 0,061 & 0,026 & 0,047 & 0,035 & 0,036 & 0,034 & 0,027 & 0,025 & 0,028 \\
\hline & 3 & 0,093 & 0,204 & 0,133 & 0,143 & 0,046 & 0,049 & 0,032 & 0,042 & 0,039 & 0,105 & 0,125 & 0,089 \\
\hline \multirow{3}{*}{$\begin{array}{l}\text { E.max } \\
1.0 \mathrm{~V}\end{array}$} & 1 & 0,041 & 0,035035 & 0,029 & 0,035 & 0,057 & 0,110 & 0,096 & 0,087 & 0,016 & 0,022 & 0,025 & 0,021 \\
\hline & 2 & 0,035 & 0,031 & 0,057 & 0,041 & 0,034 & 0,024 & 0,048 & 0,038 & 0,018 & 0,013 & 0,027 & 0,019 \\
\hline & 3 & 0,043 & 0,047 & 0,056 & 0,048 & 0,037 & 0,024 & 0,024 & 0,028 & 0,032 & 0,026 & 0,040 & 0,032 \\
\hline \multirow{3}{*}{$\begin{array}{l}\text { E.max } \\
1.0 \mathrm{R}\end{array}$} & 1 & 0,031 & 0,034 & 00,40 & 0,035 & 0,026 & 0,039 & 0,038 & 0,034 & 0,031 & 0,033 & 0,040 & 0,034 \\
\hline & 2 & 0,078 & 0,080 & 0,060 & 0,072 & 0,024 & 0,044 & 0,039 & 0,035 & 0,028 & 0,021 & 0,033 & 0,027 \\
\hline & 3 & 0,068 & 0,068 & 0,080 & 0,072 & 0,060 & 0,032 & 0,048 & 0,046 & 0,032 & 0,044 & 0,042 & 0,039 \\
\hline \multirow{3}{*}{$\begin{array}{c}\text { Zirpress } \\
0.5 \mathrm{~V}\end{array}$} & 1 & 0,030 & 0,051 & 0,008 & 0,029 & 0,083 & 0,089 & 0,064 & 0,078 & 0,062 & 0,048 & 0,042 & 0,050 \\
\hline & 2 & 0,087 & 0,028 & 0,060 & 0,058 & 0,091 & 0,041 & 0,074 & 0,058 & 0,030 & 0,038 & 0,031 & 0,033 \\
\hline & 3 & 0,033 & 0,038 & 0,032 & 0,034 & 0,064 & 0,014 & 0,058 & 0,058 & 0,075 & 0,057 & 0,050 & 0,060 \\
\hline \multirow{3}{*}{$\begin{array}{c}\text { Zirpress } \\
0.5 \mathrm{R}\end{array}$} & 1 & 0,031 & 0,079 & 0,058 & 0,056 & 0,109 & 0,082 & 0,146 & 0,112 & 0,094 & 0,073 & 0,092 & 0,086 \\
\hline & 2 & 0,052 & 0,048 & 0,035 & 0,045 & 0,043 & 0,057 & 0,074 & 0,058 & 0,044 & 0,048 & 0,012 & 0,034 \\
\hline & 3 & 0,047 & 0,038 & 0,043 & 0,042 & 0,064 & 0,014 & 0,058 & 0,045 & 0,055 & 0,058 & 0,046 & 0,053 \\
\hline \multirow{3}{*}{$\begin{array}{c}\text { Zirpress } \\
1.0 \mathrm{~V}\end{array}$} & 1 & 0,075 & 0,085 & 0,057 & 0,072 & 0,042 & 0,035 & 0,055 & 0,044 & 0,041 & 0,035 & 0,029 & 0,035 \\
\hline & 2 & 0,058 & 0,034 & 0,030 & 0,040 & 0,059 & 0,050 & 0,045 & 0,051 & 0,109 & 0,043 & 0,039 & 0,063 \\
\hline & 3 & 0,023 & 0,024 & 0,059 & 0,035 & 0,057 & 0,031 & 0,049 & 0,045 & 0,047 & 0,114 & 0,050 & 0,070 \\
\hline \multirow{3}{*}{$\begin{array}{c}\text { Zirpress } \\
1.0 \mathrm{R}\end{array}$} & 1 & 0,070 & 0,062 & 0,049 & 0,060 & 0,045 & 0,060 & 0,028 & 0,044 & 0,031 & 0,011 & 0,038 & 0,026 \\
\hline & 2 & 0,089 & 0,057 & 0,048 & 0,064 & 0,057 & 0,031 & 0,044 & 0,044 & 0,065 & 0,046 & 0,054 & 0,055 \\
\hline & 3 & 0,070 & 0,081 & 0,068 & 0,073 & 0,092 & 0,079 & 0,048 & 0,073 & 0,057 & 0,058 & 0,046 & 0,053 \\
\hline
\end{tabular}

